\title{
Influence of marijuana on driving
}

Citation for published version (APA):

Robbe, H. W. J. (1994). Influence of marijuana on driving. [Doctoral Thesis, Maastricht University]. Rijksuniversiteit Limburg. https://doi.org/10.26481/dis.19940526hr

Document status and date:

Published: 01/01/1994

DOI:

10.26481/dis.19940526hr

Document Version:

Publisher's PDF, also known as Version of record

\section{Please check the document version of this publication:}

- A submitted manuscript is the version of the article upon submission and before peer-review. There can be important differences between the submitted version and the official published version of record.

People interested in the research are advised to contact the author for the final version of the publication, or visit the DOI to the publisher's website.

- The final author version and the galley proof are versions of the publication after peer review.

- The final published version features the final layout of the paper including the volume, issue and page numbers.

Link to publication

\footnotetext{
General rights rights.

- You may freely distribute the URL identifying the publication in the public portal. please follow below link for the End User Agreement:

www.umlib.nl/taverne-license

Take down policy

If you believe that this document breaches copyright please contact us at:

repository@maastrichtuniversity.nl

providing details and we will investigate your claim.
}

Copyright and moral rights for the publications made accessible in the public portal are retained by the authors and/or other copyright owners and it is a condition of accessing publications that users recognise and abide by the legal requirements associated with these

- Users may download and print one copy of any publication from the public portal for the purpose of private study or research.

- You may not further distribute the material or use it for any profit-making activity or commercial gain

If the publication is distributed under the terms of Article $25 \mathrm{fa}$ of the Dutch Copyright Act, indicated by the "Taverne" license above, 
INFLUENCE OF MARIJUANA ON DRIVING 
- H.W.J. Robbe, Maastricht 1994

Cover design

Lay-out

R. Levigne

Production

H.W.J. Robbe

Datawyse Maastricht

CIP-DATA KONINKLIJKE BIBLIOTHEEK, DEN HAAG

Robbe, Hindrik Willem Jan

Influence of marijuana on driving / Hindrik Willem Jan

Robbe. - Maastricht: Institute for Human

Psychopharmacology, University of Limburg. - Ill.

Also publ. in commercial ed.: 1994. - Thesis Maastricht. -

With ref. - With summary in Dutch, French and German.

ISBN 90-5147-024-X bound

NUGI $735 / 746$

Subject headings: marijuana and driving. 


\title{
INFLUENCE OF MARIJUANA ON DRIVING
}

\section{PROEFSCHRIFT}

\author{
ter verkrijging van de graad van doctor \\ aan de Rijksuniversiteit Limburg te Maastricht, \\ op gezag van de Rector Magnificus, Prof. Dr. H. Philipsen, \\ volgens het besluit van het College van Dekanen, \\ in het openbaar te verdedigen op \\ donderdag, 26 mei 1994 om 14.00 uur
}

door

HINDRIK WILLEM JAN ROBBE 


\title{
Promotor
}

Prof.dr. M.A. van den Hout

\section{Co-promotor}

\author{
Dr. J.F. O'Hanlon
}

\section{Beoordelingscommissie}

Prof.dr. J. Jolles (voorzitter)

Prof.dr. G. Berghaus (Universität Köln)

Prof.dr. J.C.S. Kleinjans

Prof.dr. J.F. Orlebeke (Vrije Universiteit Amsterdam)

Prof.dr. H.M. van Praag

The research described in the dissertation was conducted at the Institute for Human Psychopharmacology, University of Limburg, Maastricht'. The marijuana studies were sponsored by the U.S. Department of Transportation; and, the 'alcohol part' in the city driving study, by the Dutch Ministry of Transport, Public Works and Watermanagement (Min. V\&W).

The author would like to thank the Faculty of Health Sciences, University of Limburg, Maastricht, for providing the opportunity to complete the dissertation; and, La Prévention Routière, Paris, for providing the translation of the summary in French. The financial support from the Institute for Human Psychopharmacology (main sponsor) for the publication of the dissertation is gratefully acknowledged. The author also thanks the following companies for their financial support: Organon International BV, Oss, SmithKline Beecham Farma BV, Rijswijk, and UCB Pharma Nederland BV, Breda. 
Aan mijn ouders

Voor Greetje, Axel en Annemoon 


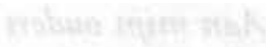

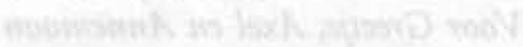

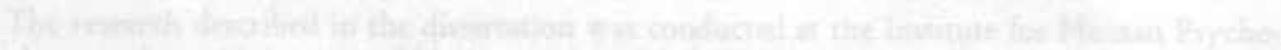

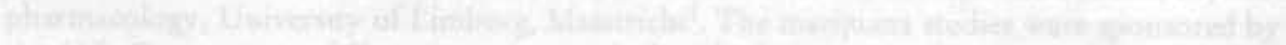

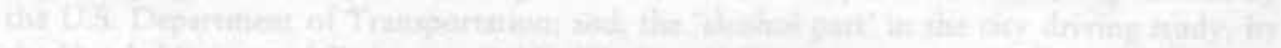

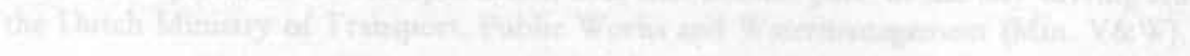

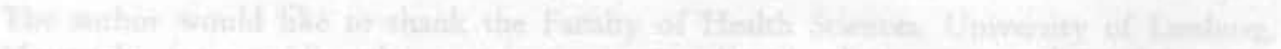

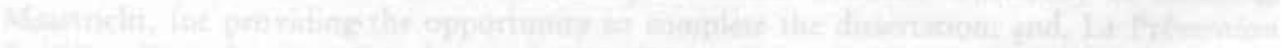

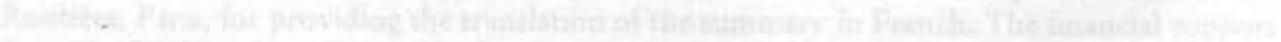

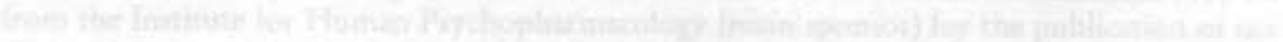

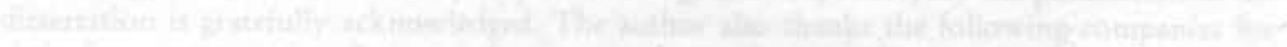
and

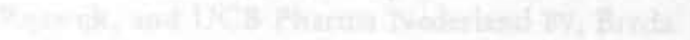




\section{CONTENTS}

\section{List of Abbreviations 9}

Chapter 1 Raison d'être and Overview 11

Chapter 2 General Introduction 13

2.1 History of Cannabis Use 13

2.2 Prevalence of Cannabis Use 16

2.3 The Cannabis Plant and its Preparations 17

2.4 Chemistry and Pharmacology 17

2.5 Effects of Cannabis Use 24

2.5.1 Acute Effects 24

2.5.2 Factors influencing the Drug's Acute Effects 30

2.5.3 Chronic Effects 37

2.5.4 Tolerance and Dependence 45

2.5.5 Therapeutic Applications 48

2.6 Summary and Conclusions 49

Chapter 3 Cannabis and Driving 52

3.1 Epidemiological Studies 52

3.2 Laboratory Studies of Skills Related to Driving 56

3.3 Driving Simulator Studies 61

3.4 Actual Driving Studies 62

3.5 General Conclusion 66

Chapter 4 General Methods 68

4.1 Description of a 4-Study Program 68

4.2 Compliance with Ethical and Legal Standards 70

4.3 Subject Recruiting 70

4.4 Marijuana Cigarettes and Smoking Procedures 72

4.5 Screening for the Presence of Other Illicit Drugs and Alcohol 73

4.6 Blood Sampling and Quantitative Analyses 73

Chapter 5 Laboratory Study to Select THC Doses 76

5.1 Introduction 76

5.2 Methods 76

5.3 Results 80

5.4 Discussion 88 
Chapter 6 Marijuana and Driving on a Restricted Highway 91
6.1 Introduction 91
6.2 Methods 92
6.3 Results 95
6.4 Discussion 106

Chapter 7 Marijuana and Driving on a Normal Highway in Traffic 111

7.1 Introduction 111

7.2 Methods 112

7.3 Results 119

7.4 Discussion 136

Chapter 8 Marijuana, Alcohol and Urban City Driving 142
8.1 Introduction 142
8.2 Methods 144
8.3 Results 151
8.4 Discussion 159

Chapter 9 General Discussion, Conclusions and Recommendations 163

9.1 Introduction 163

9.2 THC Doses 165

9.3 The Driving Tests 167

9.4 Effects of THC on Driving Performance 169

9.4.1 Results of Present Studies 169

9.4.2 Drug Plasma Concentrations and Driving Performance 170

9.4.3 Cannabis versus Alcohol and Other Psychotropic Drugs 171

9.4.4 Why are THC's Effects on Driving Performance relatively Small? 173

9.4.5 Concluding Remarks 176

9.5 Summary of Conclusions 178

9.6 Summary of Recommendations 179

Summary 180

Samenvatting 188

Résumé 197

Zusammenfassung 207

References 217

Dankwoord 233

Curriculum Vitae 235 


\section{LIST OF ABBREVIATIONS}

11-OH-THC 11-hydroxy- $\Delta^{9}$-tetrahydrocannabinol

\section{$\Delta^{9}$-THC $\quad \Delta^{9}$-tetrahydrocannabinol}

BAC blood alcohol concentration

CBD cannabidiol

CBN cannabinol

CFF critical flicker/fusion frequency

CNS central nervous system

$\mathrm{CO}$ carbon monoxide

CS-C curve surface in body sway test with eyes closed

CS-O curve surface in body sway test with eyes open

CTT critical tracking test

$\mathrm{CV}$ coefficient of variation

ECG electrocardiogram

EEG electroencephalogram

ETOH ethanol

IBI interbeat interval time

FDA U.S. food and drug administration

GC/MS gas chromatography/mass spectrometry

LSD lysergic acid diethylamide

(M)ANOVA (multivariate) analysis of variance

PWR-HR spectral measure of heart rate variability (between 0.07 and $0.14 \mathrm{~Hz}$ )

RT reaction time

SD standard deviation

SDLP standard deviation of lateral position

SDSP standard deviation of speed

SDST standard deviation of steering wheel angle

SE standard error

SED standard error of the mean difference

SP mean speed

THC tetrahydrocannabinol

THC-COOH 11-nor- $\Delta^{9}$-tetrahydrocannabinol-9-carboxylic acid 


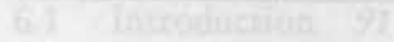

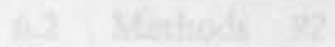

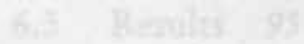

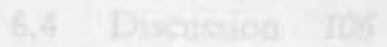

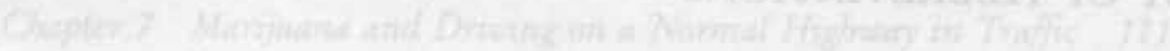

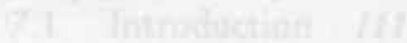

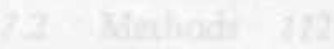

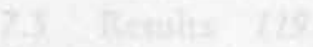

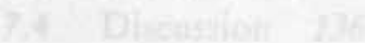

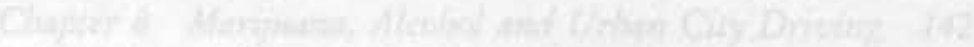

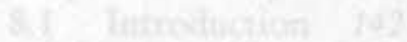

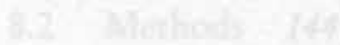

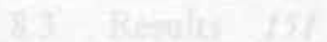

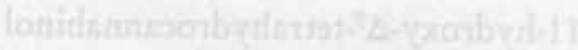

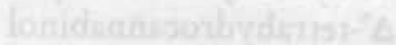

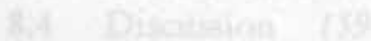

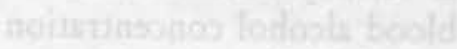

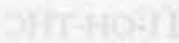

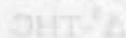
Coifistidinitit: 3inte ciniti

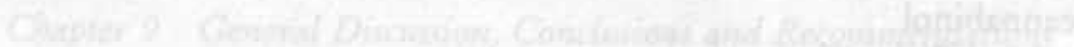
intat

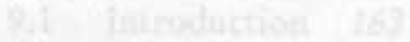

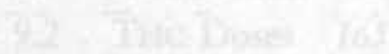

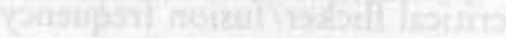
$¥=$

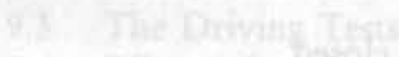

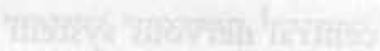

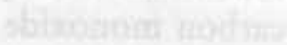
197.7.

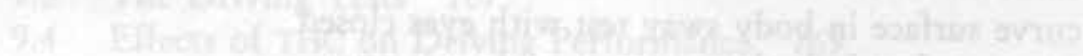

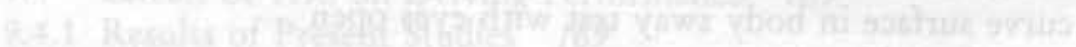

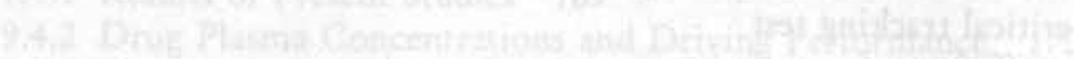

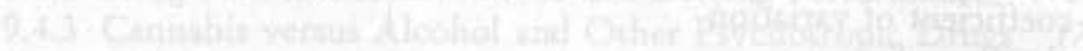

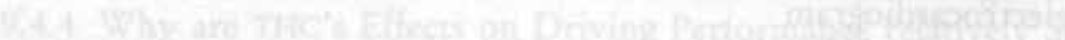

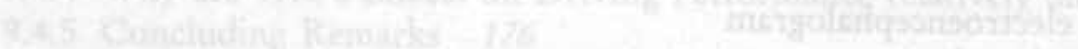

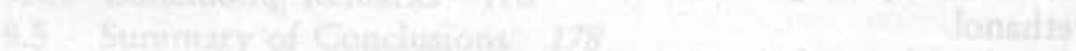

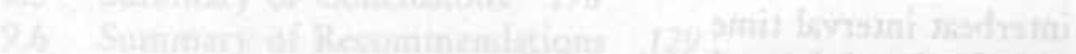

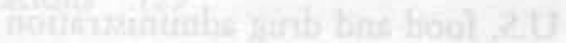

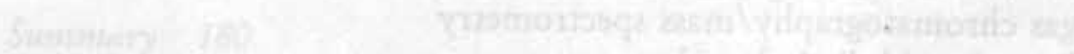

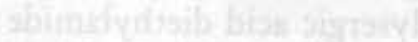

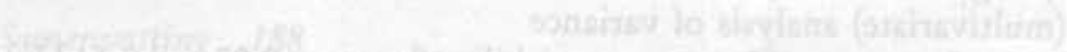
atwighath

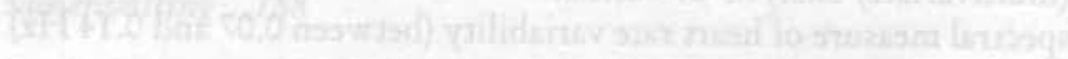

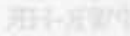

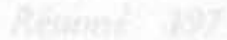

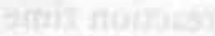

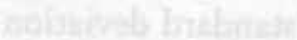

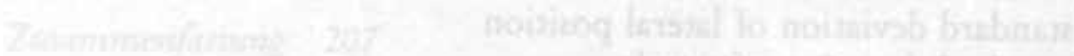
(ats Giscis

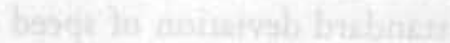
502

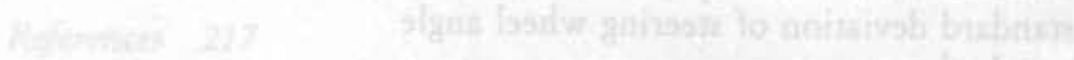
TaMik tis

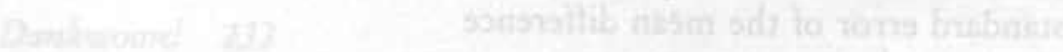
orat

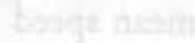

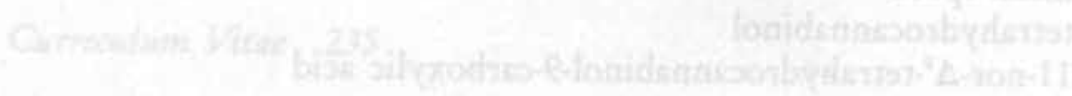




\section{CHAPTER 1}

\section{RAISON D'ÊTRE AND OVERVIEW}

Every psychoactive drug exerts its action within the central nervous system (CNS). Psychoactive drugs taken for medicinal purposes not only produce desired therapeutic effects but also unwanted side effects, such as sedation and memory impairment, due to the complex interaction of numerous neurotransmitter systems within the brain and the drugs' lack of selectivity for one apart from the others. It is in the interest of road safety to determine which drugs seriously interfere with brain functions to identify those that are incompatible with safe driving performance. It is estimated that about $10 \%$ of the more than 6500 registered medicinal drugs in the Netherlands have the potential to impair driving performance (Weseman et al., 1989).

Alcohol is the cardinal example of a licit, nonmedicinal psychoactive drug that adversely affects driving performance and causes many fatal road crashes. The relationship between dose and effect is unequivocal: rising blood concentrations produce exponential deterioration in experimentally measured driving performance (Louwerens et al., 1987) and the risk of causing a fatal traffic accident as determined by epidemiological surveys (Borkenstein et al., 1974). Therefore, nearly all countries have adopted a legal limit of the blood alcohol concentration (BAC) for driving-under-the-influence prosecution.

Cannabis (e.g. marijuana and hashish) is the best-known example of an illicit, nonmedicinal psychoactive drug. Since the 1960 s, when cannabis smoking became commonplace in Western societies, much research has been executed to determine cannabis' adverse and also beneficial effects on safety, health, personality, behavior, etc. This drug's effects on driving performance have also been investigated, both by epidemiological and by experimental studies. Both had their limitations as will be described in Chapter 3. Until now, epidemiological studies failed to show a reliable relationship between cannabis use and risk of a motor vehicle accident. These failures are mainly due to the absence of proper control groups and the high incidence of combined alcohol and cannabis use among drivers responsible for accidents. Experimental studies on cannabis and driving performance have mainly been executed in driving simulators and on closed circuits. Only one study reported previously in the literature measured the effects 
of marijuana smoking on actual driving performance (Klonoff, 1974). The results of these studies are not as unequivocal as those involving alcohol. Nonetheless, they generally failed to show drastic driving impairments after cannabis smoking.

The question if cannabis adversely affects driving performance is obviously highly relevant but the answer is subject to controversy. Some hold that cannabis is a relatively minor factor in traffic accidents (e.g. Mason and McBay, 1984; Gieringer, 1988) or that the magnitude of accident risk following cannabis smoking remains obscure (e.g. Peck et al., 1986). Others, including scientists and politicians, remain convinced that cannabis is a serious public safety hazard (e.g. Milner, 1977; Moskowitz, 1985). Their opinion is mainly based upon results from laboratory experiments showing adverse effects of the drug on cognitive and psychomotor performance, but also on anecdotal reports of accidents. Given the societal relevance of the issue and the persistence of the controversy, it was decided to investigate cannabis' effects on driving performance in the most valid ecologically environment that is available, i.e. in actual traffic on the public road.

This dissertation describes the results of one laboratory and three actual driving studies. In all driving studies, subjects operated a specially instrumented vehicle on a public road after smoking marijuana. In the first study, they drove on a primary highway closed for other traffic; in the second, on a primary highway in the presence of other traffic; and, in the third, through the city of Maastricht, again in the presence of other traffic. Together, these studies should provide an answer to the questions addressed by this dissertation: does marijuana smoking impair driving performance, and if so, to what magnitude in comparison with other psychoactive drugs.

Chapter 2 provides a general overview of cannabis. It starts with the history of cannabis use and goes on to describe its current usage prevalence. Then a short description of the plant and its preparations is given, followed by a more extensive part on the drug's chemistry and pharmacology. The concluding part of the chapter is dedicated to an overview of the acute and chronic effects of cannabis. After this general introduction, Chapter 3 concentrates on the literature pertinent to the effects of cannabis on driving. Both epidemiological surveys of users' involvement in traffic accidents and empirical studies measuring the drug's influence on skills related to driving are reviewed. This provides the justification for the experimental research reported in the dissertation. Chapter 4 describes methods that were common to all studies. These are successively reported in Chapters 5-8. The dissertation concludes in Chapter 9 with a general discussion, conclusions from the studies' results, and recommendations concerning further research requirements. 


\section{CHAPTER 2}

\section{GENERAL INTRODUCTION}

This chapter provides a general overview of cannabis, starting with a historical perspective which is followed by a description of its current usage prevalence. Then a short introduction to the plant and its derivatives is given, followed by an extensive review of the drug's chemistry and pharmacology. The concluding part is dedicated to an overview of the acute and chronic effects of cannabis use. Finally, the drug's therapeutical applications are shortly summarized.

\subsection{History of Cannabis Use}

Throughout recorded history man has shown a great interest in Cannabis sativa, also known as Indian hemp, which is an herbaceous annual. That interest extends backward for about twelve thousand years (Abel, 1979). The ancient Chinese used cannabis as an ingredient in clothes and ropes. The Greeks became familiar with this application of cannabis during the sixth century BC. At first they obtained cannabis from Milesion colonies, but by the third century BC, primarily from the Rhone valley in France. The Romans were aware of the tensile strength of cannabis rope and used it extensively for naval construction. Early in American history the plant was widely cultivated for its commercially useful fiber, beginning in Jamestown, Virginia, in 1611 (Grinspoon and Bakalar, 1992).

Cannabis has long been used as a medicine in India, China, the Middle East, Southeast Asia, South Africa, and South America (Grinspoon and Bakalar, 1993). The earliest description of the drug, however, dates back to the twenty-eight century BC (Abel, 1979). The Chinese emperor Shen-Nung described hundreds of drugs, among them cannabis, in an herbal (the Pen Ts'ao) that eventually became the standard Chinese compendium of medicines. Cannabis was recommended for relief or recovery from rheumatic pains, constipation, malaria, 'absentmindedness', and female disorders. Chinese physicians discovered the painkilling properties of cannabis and the drug was applied as an analgesic during complicated surgical procedures in the second century AD. In India, the anxiolytic and euphoric properties of cannabis were discovered some time between 
2,000 and $1,400 \mathrm{BC}$ when it was used to concoct one of India's favorite beverages. It was further recommended for quickening the mind, lowering fevers, inducing sleep, curing dysentery, stimulating appetite, improving digestion, relieving headaches, and curing venereal disease. The medicinal effect of cannabis has also been recognized in Western societies, some millennia later. During its heyday, from 1839 to the turn of the century, more than one hundred papers appeared in the Western medical literature (Grinspoon and Bakalar, 1992). Cannabis was prescribed for various diseases and discomforts, such as insomnia, asthma, coughing, fatigue, rheumatism, delirium tremens, and pain with menstruation. It was included in the United States Pharmacopeia from 1850 to 1942 (Himmelstein, 1983). At the turn of the twentieth century, however, the medicinal use of cannabis waned, mainly due to the development and introduction of synthetic medicines. Yet there is at present renewed scientific interest in the medicinal applications of cannabis due to the identification of the active cannabinoids and the development of synthetic analogs. Research efforts in this area will very likely increase dramatically in the very near future because of the recent discovery of cannabis receptors and a potential endogenous ligand in the human brain (Section 2.4).

Among other medicinal properties of cannabis, the Chinese recognized its euphoric and mind-altering potentials. Then as now opinions regarding these effects were divided. Some were convinced that cannabis would destroy society and others believed it would be a boon to mankind. The matter was resolved about $600 \mathrm{BC}$ by the Taoist condemnation of anything that enfeebled the body. Afterwards both medicinal and recreational usages of the drug disappeared from China (Abel, 1979). In Arab lands, hashish became a commonly used drug for its euphoric properties by the thirteenth century AD. Though Cairo authorities disapproved of and tried to suppress cannabis use, it had become too ingrained to be eradicated. One tale of hashish' effects dating from those times played a major role in generating the drug's poor reputation among the general public, namely the story of the bashshashim which means hashish-eaters. The Arabic name, from which the English word assassin originates, was given to a group of moslem terrorists who murdered Christians at the time of the Crusades. Although they were users of hashish, as many others in the Arab world were, Abel states that there is no evidence that the terrorists took the drug to fortify themselves for errands of death. The Arabs themselves have rarely regarded hashish as a drug leading to violence and crime. Indeed, cannabis may suppress violent behavior because of the mild lethargy it induces (Grinspoon and Bakalar, 1992). Nevertheless, this tale was readily used for anti-cannabis campaigns over subsequent centuries.

Although cannabis was primarily used by the Europeans for making rope during the Middle Ages, some were already aware of its inebriant properties. Sorcerers and witches were accused of incorporating cannabis in drugs. Pope 
Innocent VIII in 1484 included cannabis in the list of satanic compounds. As the euphoric properties of the drug became more widely known a group of French writers formed the 'Club des Hashischins' in Paris in the 1850s. They were fascinated by the stories of the mind-altering effects of hashish that came in the wake of Napoleon's retreat from Egypt and wrote novels under its influence, e.g. Artificial Paradises by Charles Baudelaire (1860). North Americans became aware of the drug through Bayard Taylor, writer and traveler best known for his translation of Goethe's Faust. He tried it during his visit to Egypt in 1854 and wrote two books of which The Land of the Saracens (1855) contains the most comprehensive description of his hashish experiences. Hashish acquired a lurid reputation through these writings and use of the drug waned considerably in the Western world.

A revival of cannabis use was seen in the United States with the passage of the Eighteenth Amendment in 1920 which prohibited the sale of alcohol. Cannabis began to be brought across the border from Mexico and smoked for pleasure by minority groups. The common name for any part of the cannabis plant became marijuana after the Mexican Spanish word maraguanquo meaning an inebriant plant (Maykut, 1985). Still, very little was known about the drug. Little attention was paid to marijuana use until Commissioner Ansinger of the U.S. Federal Bureau of Narcotics and Dangerous Drugs - according to Abel, in an effort to dissuade Congress from reducing the bureau's budget - started a media campaign against marijuana in the 1930s. The Bureau of Narcotics and Dangerous Drugs circulated stories about crimes attributed to marijuana use and later used the published stories as evidence in support of its policies. Cannabis was banned in the USA by the 1937 Marijuana Tax Act which required anyone using it to register and pay a tax of one dollar an ounce for industrial or medical purposes and of hundred dollars an ounce for unregistered transactions having any other purpose (Grinspoon and Bakalar, 1993). Although primarily designed to prevent 'recreational' use, it also caused cannabis to lose its medical status. In the Netherlands, cannabis was proscribed by the Opium Law of 1928 when it was still rarely used.

The second revival of marijuana use occurred during the 1960s, at the time of the 'flower power' movement when a large number of young people began to use marijuana recreationally. Many of the American users during this period were Vietnam War veterans who were introduced to cannabis and other intoxicating drugs while on duty in Southeast Asia. Cannabis' popularity became widespread, both in the United States and Western Europe, not only in lower social classes and minority groups but throughout all levels in society. Cannabis has ever since become the most widely used illegal drug in most of these countries. As a consequence, public and scientific interest in the plant and its usages increased dramatically. About 8,000 references - dealing not only with the psychic and physical effects of the drug but also with other topics such as 
industrial usage, cultivation, history, and legal status - to papers published prior to 1978 are collected in Abel's (1979) Comprehensive Guide to the Cannabis Literature. The number of articles has since increased by many thousands - in the years 1990-1993, more than 1,200 references to scientific articles can be found and it is very unlikely that the rate of publications will decrease after recent breakthrough discoveries concerning the drug's pharmacology.

\subsection{Prevalence of Cannabis Use}

Cannabis is by far the most widely used illicit drug in most countries all over the world, including Nigeria (Morakinyo, 1983), Australia (Rankin, 1985), Israel and France (Kandel, 1984), Greenland (Pedersen, 1992), Canada (Campbell and Svenson, 1992), and Spain (Alvarez et al., 1992). Unlike alcohol and tobacco that are used by people of all ages, a preference for cannabis predominates among the young people who apparently use it less when growing older.

In the United States, marijuana usage prevalence peaked in the late 1970s and has been declining ever since. But even there marijuana is the most commonly used illicit drug (Jessor et al., 1986; Johnston et al., 1992). The most recent data about marijuana usage prevalence are available from the $17^{\text {th }}$ National Survey of American High School Seniors, and the $12^{\text {th }}$ National Survey of American College Students (Johnston et al., 1992). Lifetime prevalence among high school seniors declined from $60.3 \%$ in 1980 to $36.7 \%$ in 1991 ; and, among college students, from $65.0 \%$ to $46.3 \%$. Thirty-day prevalence declined in both groups from about $34 \%$ in 1980 to $14 \%$ in 1991 . While $9.1 \%$ of high school seniors and $7.2 \%$ of college students admitted daily use of marijuana in 1980 , only about $2 \%$ of both groups did in 1991.

In the Netherlands, prevalence data have been obtained by the Youth Health Care from students in grades 7 and 8 of primary education and all levels of secondary education in 1984, 1988, and 1992 (Van der Wal, 1985; Plomp et al., 1990; De Zwart et al., 1993). A three-fold increase in cannabis prevalence was found among the total group during this period. Lifetime prevalence increased from $4.8 \%$ in 1984 to $13.6 \%$ in 1992 ; thirty-day prevalence, from $2.3 \%$ to $6.5 \%$. Considering only those of 18 years or older, lifetime prevalence of cannabis increased during this period from $15.7 \%$ to $46.8 \%$ among males, and from $13.5 \%$ to $22.0 \%$ among females. Thirty-day prevalence increased from $9.2 \%$ to $17.0 \%$ among males and decreased from $8.1 \%$ to $6.1 \%$ among females.

In short, relatively more young Americans formerly used and still use cannabis than their Dutch counterparts, but the disparities between lifetime and current use prevalences are narrowing due to opposite trends in the two countries. It is perhaps important to note in this context that possession of cannabis is prohibited by law in The Netherlands, as in the United States. The seriousness 
of the offense is, however, determined by the amount found in the Dutch user's possession and prosecution is unlikely to occur when that is less than $30 \mathrm{~g} \mathrm{(1} \mathrm{oz).}$

\subsection{The Cannabis Plant and its Preparations}

There is only one species of the genus Cannabis, Cannabis sativa, with two subspecies of sativa and indica, each with wild and cultivated varieties (Small and Cronquist, 1976). The subspecies sativa has been cultivated for fibre obtained from its stem to produce rope and linen and for its seeds to produce birdseed and oil for quick-drying paint. The principal interest in cannabis, however, has been in the sticky golden resin that covers the flowers and top leaves of the subspecies indica. The resin contains the psychoactive chemical compounds known as cannabinoids. The cannabinoid content of the drug varies widely depending on type of plant, climate, soil, cultivation, and part of plant. The highest concentration of cannabinoids is found in the flowering tops of the plant, followed by its leaves. Very small quantities of cannabinoids are found in the stem and roots of the plant, and none in the seeds. Female plants produce higher concentrations of cannabinoids than their male counterparts.

Roughly speaking, three grades of drug preparations exist, identified by the Indian names bhang, ganja, and charas (Maykut, 1985; Grinspoon and Bakalar, 1992). Bhang is obtained from dried leaves and flowering tops of uncultivated plants and has a low resin content. Ganja is made from the dried leaves and flowering tops of carefully selected cultivated plants and contains larger quantities of resin with a higher quality. Charas is prepared from the resinous exudate itself, obtained from the flowering tops of mature cultivated plants. The first two grades are known as marijuana, the latter as hashish which is five to ten times stronger than marijuana. Hashish oil, a concentrated extract of hashish sometimes mixed with alcohol, is even stronger. Many street names of the drug exist, such as: grass, hemp, pot, rope, weed, and sinsemilla. The latter, literally meaning 'without seeds', is a potent and popular street preparation which results from careful manicuring of the unfertilized flowering tops of the female plant. The plant is normally cut, dried, chopped, and incorporated into cigarettes with or without tobacco. The drug can also be smoked in a waterpipe, chewed, prepared as tea, or eaten in baked goods.

\subsection{Chemistry and Pharmacology}

The hemp plant contains more than 400 chemical compounds. More than 60 , the cannabinoids, are specific to that plant and belong to the terpenophenolic chemical class (Turner et al., 1980). The term cannabinoid is used for the typical 
$\mathrm{C}_{21}$-compounds present in Cannabis sativa and includes their analogs and transformation products. Cannabinoids are very lipid soluble and water insoluble due to nitrogen lack in the molecule. The majority of the cannabinoid products are pharmacologically inactive. The main active ingredients of cannabis are cannabinol (CBN), cannabidiol (CBD) and several isomers of tetrahydrocannabinol (THC). The constituent primarily responsible for the physiological and psychological effects of cannabis is $\Delta^{9}$-THC (Gaoni and Mechoulam, 1964). Its pharmacological activity is stereoselective, the (-)-trans isomer of $\Delta^{9}$-THC being 6-100 times more potent than the (+)-trans isomer dependent upon the species as well as the pharmacological test (Dewey et al., 1984). Another physiologically active isomer, $\Delta^{8}$-THC, is about equipotent as $\Delta^{9}$-THC but is of trivial practical importance because of the minute amounts available in the plant material. Unless stated otherwise, the abbreviation THC is hereafter used to refer to $\Delta^{9}$-THC. CBD is devoid of psychoactive properties (Karniol and Carlini, 1973; Belgrave et al., 1979) whereas CBN possesses about $1 / 10$ the potency of THC in man (Hollister, 1973; Perez-Reyes et al., 1973). Although most cannabinoids do not posses psychoactive properties themselves, it is quite possible that they interact with THC in a synergistic, additive, or even antagonistic manner. It has been suggested, for example, that CBD delays both the onset and offset of THC's effects, and either antagonizes the stimulatory or potentiates the depressant properties of THC (e.g. Karniol and Carlini, 1973).

Since THC is the most prominent psychoactive compound in cannabis its concentration mainly determines the psychotropic activity of the drug. It should be mentioned that the THC in the plant material is predominantly in the form of a THC acid derivative. Only after heating, the acid is instantly and totally decarboxylated to THC itself. Therefore, hemp should first be heated before eaten or drunk. When smoked, the acid derivative is totally converted to THC and only THC itself appears in the smoke. The combined concentration of THC and THC acid in marijuana cigarettes varies in the United States from about 0.5 to $11 \%$ (Jaffe, 1990). In The Netherlands, seized hemp material usually contains about $10 \%$ THC, though it may range from 5 to $15 \%$ and, in exceptional cases, to $25 \%$ (Dutch Forensic Laboratory, personal communication).

Cannabis is usually smoked as a $0.5-1 \mathrm{~g}$ marijuana cigarette. Hashish, having a far greater potency, is often mixed with tobacco before smoking. The characteristic odor of cannabis is not due to the cannabinoids but to volatile essential oils. Tobacco and marijuana smoke are quite similar in many respects. The most obvious difference is that nicotine is present in tobacco while THC, CBD, and CBN are present in the marijuana smoke condensate (Harvey, 1984). Compared to a nicotine cigarette, smoking a marijuana cigarette delivers greater amounts of the carcinogenic products, tar and benzopyrene, to the smoker's mouth, and leads to greater retention of the inhaled tar in the lung as well as greater boosts in blood carboxyhemoglobin (Salemink, 1984; Wu et al., 1988; Tashkin et al., 1991). 
Davis et al. (1984) studied the smoking characteristics of marijuana cigarettes under smoking-machine conditions. They found that the humidity of the marijuana cigarette - humidification is often used to moderate 'harshness' upon smoking - and type of cigarette paper used do not alter the amount of THC delivered to mainstream smoke. When a complete marijuana cigarette was consumed in a single puff, $69 \%$ of the available THC was recovered in the mainstream smoke. Apparently, about $30 \%$ of the THC was destroyed by pyrolysis. When they simulated the puff duration and puff volume that many marijuana smokers practice, only 16 to $19 \%$ of the THC was found in the mainstream smoke condensate. Others have found numbers in the range of 10 to $25 \%$ (summarized by Agurell et al., 1986), the former being more characteristic of light users, the latter of heavy users. When administered orally, THC's bioavailability is only about $6 \%$ due to its sensitivity to acidic gastric juice and first pass metabolism in gut and liver.

The THC dose required to produce different pharmacological effects in humans ranges from 2 to $22 \mathrm{mg}$ for smoking and 20 to $90 \mathrm{mg}$ when THC is administered orally (Martin, 1986). A profound psychological 'high' can be experienced after smoking a cigarette containing $10 \mathrm{mg}$ THC. If only $10-20 \%$ of the available THC enters the circulation when the drug is smoked, the THC dose range would be reduced to $0.2-4.4 \mathrm{mg}$. Animal studies showed that the THC level in the brain is surprisingly small, at most $1 \%$ of the administered dose at peak concentration (Agurell et al., 1986). Assuming a similar distribution in humans, only $2-44 \mu \mathrm{g}$ THC would be expected to penetrate the brain with less than $20 \mu \mathrm{g}$ being necessary to produce a profound 'high'.

Although the required dose to produce pharmacological effects is quite small, huge doses of cannabinoids can be administered without causing death. The lethality of drugs is normally expressed in the $\mathrm{LD}_{50}$, the dose that will cause death in $50 \%$ of the humans or animals taking it. The $\mathrm{LD}_{50}$ in humans is, however, not known because lethal effects of overdose by humans are nonexistent or rare. The toxicity of drugs is expressed as a therapeutic ratio of safety factor which is the ratio of lethal to effective dose. On the basis of animal studies estimates of the safety factor of THC in humans vary from 4,000 (Abel, 1979) to 40,000 (Grinspoon and Bakalar, 1993). The safety factor for many cancer chemotherapy drugs is only 1.5 and, for alcohol, 4 to 10 . In this respect, cannabis is a remarkably safe drug.

The metabolism of THC is exceedingly complex and more than 80 metabolites are known to be formed in man. After marijuana smoking or THC injection, the first metabolite, 11-hydroxy- $\Delta^{9}$-THC (11-OH-THC) is formed in the lungs and liver. Its peak concentration in relation to the parent compound's is about 1:10-20 (Wall et al., 1983; Huestis et al., 1992). After oral THC the ratio is about 1:1-2. Because this metabolite's psychotropic activity is equipotent to the parent's, it contributes to the total cannabis effect, particularly when the drug is ingested. 
11-OH-THC is converted by the liver into a number of inactive metabolites. The primary pathway leads to the formulation of 11-nor- $\Delta^{9}$-THC-9-carboxylic acid (THC-COOH), the most abundant inactive metabolite in plasma, and in urine where it is partially conjugated.

Plasma concentrations of THC peak during the smoking process (Perez-Reyes et al., 1982; Huestis et al., 1992; Mathew et al., 1993) and decline exponentially in two sequential phases. In the initial distribution $(\alpha)$ phase, the drug passes rapidly out of the plasma and into highly perfused fatty tissues such as liver, lung, kidney, and spleen. Although the brain also receives a high blood flow it is, on the basis of animal studies, generally assumed that only a very small proportion of THC will pass the blood-brain barrier. The first phase is followed by a much more prolonged elimination $(\beta)$ phase that contributes to the accumulation of THC in poorly perfused tissues and wherein THC is redistributed from tissue into blood, metabolized and excreted in urine and feces. The $\alpha$-phase half-life $\left(t_{1 / \alpha \alpha}\right)$ is only about 30 minutes, whereas that of the $\beta$-phase $\left(\tau_{r_{2} \beta}\right)$ was estimated to vary between 18 and 36 hours depending upon the individual (Wall ct al., 1983; Chiang and Barnett, 1984), which would be in the same range as many psychoactive drugs such as amitriptyline, haloperidol, and nitrazepam (Agurell et al., 1986). Yet recent studies provided evidence that the earlier reported terminal elimination half-lives were underestimated due to short sampling times (Johansson et al., 1988, 1989a). In the latter study, ten marijuana smokers who habitually smoked at least one cigarette per day were asked to smoke four cigarettes during a two-day period delivering a total 'loading dose' of approximately $56 \mathrm{mg}$ THC, and then to abstain from cannabis use for four weeks. Eight subjects smoked marijuana containing deuterated THC, two smoked unlabeled THC. Plasma samples were analyzed using gas chromatography followed by mass spectrometry (GC/MS), with an improved detection limit of $20 \mathrm{pg} / \mathrm{ml}$. The decline in THC's plasma concentrations followed the same pattern as previously observed and were less than $1 \mathrm{ng} / \mathrm{ml}$ on the second day of abstinence in all subjects except one. THC remained detectable between 10 to 15 days in the subjects who had smoked deuterated THC, and the terminal elimination half-lives ranged from 2.6 to 7.2 days (mean $\pm S D=4.3 \pm 1.6$ ). THC remained detectable for 24 and 28 days in the orher two subjects, with half-lives of 9.6 and 12.6 days, respectively.

The peak plasma concentration of 11-OH-THC is achieved within 15-30 minutes and from there declines according to essentially the same pharmacokinetic profile as its parent. The rise in THC-COOH's plasma concentration is relatively slow, reaching an ill defined peak in different individuals within 1-2 hours. Its elimination follows a monoexponential profile with various individuals showing $t_{i, 3}$ 's from less then 24 to more than 72 hours. Given the above evidence for prolonged terminal elimination half-lives for THC, one should not be surprised that future studies may show that these periods were also underestimated. 
Despite what might be expected from the elimination half-lives, there is hardly any accumulation of THC in the blood. Nahas et al. (1981) demonstrated that after repeated intramuscular injections of $\Delta^{8}$-THC in rats accumulation does occur in neutral fat and liver but not in blood and brain. In man, THC was found in fat biopsies four weeks after drug administration (Johansson et al., 1989b) and THC metabolites were present in urine for several days to more than a month after the cessation of marihuana use (Hollister and Kanter, 1980; Dackis et al., 1982; Ellis et al., 1985). Therefore, the presence of cannabinoids in urine provides little information concerning the time of last drug use. Urine screening may be sufficient in epidemiological studies investigating the extent of use of cannabis in selected population groups. But for an indication of intoxication in studies to show the role of cannabis as a cause of road traffic accidents, plasma samples need to be taken and assayed for THC. Radioimmunoassay is widely applied in qualitatively analyzing urine samples, GC/MS is the procedure of choice when plasma samples need to be quantitatively analyzed (Cook, 1986).

Though peak concentrations of THC are achieved during smoking, the maximum psychological effect (or 'high') occurs 15-30 minutes after its cessation. This temporal dissociation between plasma concentration and effect suggests that brain concentrations increase as plasma concentrations decrease, possibly due to slow penetration of the blood-brain barrier, slow distribution within the brain, and a lag-time in pharmacological activity (Agurell et al., 1986). Both peak plasma concentrations and maximum psychological 'high' are roughly proportional to the inhaled THC dose, but correlations between these parameters measured simultaneously at times 3-240 min after the cessation of smoking are, albeit significant, not especially strong. For example, Ohlsson et al. (1980) found the overall correlation for repeated measurements obtained from 11 experienced smokers to be $r=0.53$. After four hours, the psychological 'high' had vanished and plasma THC levels were very low. Great interindividual variation exists in plasma levels of THC after smoking and this variation cannot be attributed to the regularity of cannabis use (Lindgren et al., 1981). It was even noted when a paced smoking protocol was used (Huestis et al., 1992).

Available evidence leads to the conclusion that it is usually impossible to predict the psychological effects of THC from its determination in a single plasma sample. But this is not the same as saying that no biological index of cannabis intoxication will ever be found. One possible candidate is THC's inactive metabolite THC-COOH. The relationship between this metabolite's plasma concentration and the perceived 'high' after cannabis smoking has never been defined, although both parameters were measured in the study by Perez-Reyes et al. (1982). Peak and time integrated $\mathrm{THC}-\mathrm{COOH}$ concentrations were proportional to the administered THC doses. Interestingly, the occurrence of the peak $\mathrm{THC}-\mathrm{COOH}$ concentration coincided in time with the subjects' report of maximum 'high'. The authors failed, however, to measure, or at least report, the correlation 
between plasma THC-COOH concentration and subjective feelings because of the metabolite's pharmacological inactivity. Yet this coincidence might signify a useful epiphenomenal correlation. This possibility was repeatedly explored in this program.

Until about a decade ago, the mechanisms by which THC exerts its effects on the CNS were poorly understood. One of the problems was that cannabinoids affect almost every system in which they are examined (Dewey, 1986). THC partitions into biological membranes and affects many membrane related functions including neurotransmitter uptake systems, enzymes, and receptors. It has long been hypothesized that cannabinoids alter membrane properties nonspecifically without interacting with any specific membrane receptor (e.g. Roth and Williams, 1979). But during the 1980 s evidence for the possible existence of receptors slowly emerged. Both the stereoselectivity of cannabinoids (Dewey et al., 1984) and the observation that modest structural modifications of the THC molecule results in profound changes in behavioral activity (Razdan, 1986) argued for an receptor-mediated mechanism of action. Martin (1986) reviewed the literature on the cellular effects of cannabinoids and was tempted to think of receptor-mediated effects because unique pharmacological effects occur at reasonably low drug concentrations. The diverse effects of cannabinoids on enzymes as well as neurotransmitter and opioid receptors at high concentrations would seem to be produced by a general perturbation of membranes.

Understanding of the mechanism of action of cannabinoids rapidly progressed by using a class of high-potency synthetic compounds originally developed for their analgetic properties such as levonantradol, its active metabolite desacetyllevonantradol, and CP-55,940 (Johnson and Melvin, 1986). Howlett and her colleagues (Howlett, 1985, 1987; Howlett et al., 1988) showed that centrally acting cannabinoids attenuate cyclic AMP accumulation by inhibition of adenylate cyclase, and that the ability of inhibition caused by a series of synthesized cannabinoids occurred in a reversible, cell type-specific, potent, and stereoselective manner. The same group of researchers identified and characterized a highaffinity, stereoselective, pharmacologically distinct receptor in membranes from rat brain by using tritium-labeled CP-55,940 (Devane et al., 1988). These receptors are coupled to adenylate cyclase via the inhibitory guanine-nucleotide protein, $G_{i}$, and thereby inhibit cyclic AMP production. Furthermore, they are involved in the regulation of $\mathrm{K}^{+}$and $\mathrm{Ca}^{2+}$ currents in neuronal cells (Abood and Martin, 1992).

Herkenham et al. (1990) localized cannabinoid receptors in brain sections of rat, guinea pig, dog, and rhesus monkey, as well as in human brains obtained from people who died from nonneurological disorders. Autoradiography using $\left[{ }^{3} \mathrm{H}\right] \mathrm{CP}-55,940$ revealed similar distributions of binding in all species with the greatest abundance of binding sites in the globus pallidus, substantia nigra pars reticulata, and the molecular layers of the cerebellum and the hippocampal 
dentate gyrus. Receptors were also dense in the cerebral cortex, striatum, and the remainder of the hippocampal formation. Comparatively sparse binding was found in lower brain stem areas and spinal cord. Identical receptors were also found in human testis (Gérard et al., 1991), and human spleen, tonsils and peripheral blood leukocytes (Bouaboula et al., 1993), albeit in smaller concentrations than in the brain. Cannabinoid receptor genes have now been cloned in both rat (Matsuda et al., 1990) and human (Gérard et al., 1991), and the deduced amino acid sequences exhibit more than $97 \%$ identity. The latest breakthrough is the isolation of a potential endogenous ligand for the cannabinoid receptor (Devane et al., 1992). The compound was named 'anandamide' from the Sanskrit word ananda, meaning bliss, and from its chemical name, arachidonylethanolamide.

The single essential commonality of recreational and abused drugs is that they all act on the brain reward system. It is hypothesized that this action results in the 'high', 'rush' or 'hit' sought by its users (Gardner and Lowinson, 1991). Herkenham et al. (1991) failed to discover cannabinoid receptors on the dopaminergic neurons comprising the brain 'reward' system, suggesting that cannabinoids do not directly act there. Animal studies showed, however, that THC lowers brain reward thresholds in the medial forebrain bundle, thereby enhancing dopamine release in the reward system (Gardner et al., 1988; Gardner and Lowinson, 1991). Both effects were reversed by naloxone, an opiate receptor antagonist, suggesting that THC acting at its own receptor causes the release of an endogenous opioid which in turn acts upon $\mu$ or other receptors causing the euphoric effect. Since THC is not itself an opioid receptor agonist one should not expect it to produce the same dependency and withdrawal syndrome upon abstinence as if it were. Nonetheless the modestly euphoric and anxiolytic effects of releasing an endogenous opioid might be something heavy cannabis users wish to perpetuate. They could become psychologically dependent upon this process as the means for coping with stress or simply enriching an objectively dull life.

The recent major steps forward in understanding the pharmacology of cannabinoids tempted many investigators (Herkenham et al., 1990; Gérard et al., 1991; Abood and Martin, 1992) to speculate on the causal role cannabinoid receptors play in the multiplicity of effects observed after ingestion or inhalation of cannabinoids. The high density of receptors in the cortex may explain the diverse cognitive effects found after cannabis smoking (below). The hippocampus plays a crucial role in memory consolidation so it should come as no surprise that THC affects short-term memory. Receptors in the basal ganglia and cerebellum may explain the effects of cannabinoids on movement control; and, those in human testis, the depression of reproductive functions observed after chronic cannabis use. The low toxicity of cannabinoids probably reflects the paucity of receptors in medullary nuclei that mediate respiratory and cardiovascular functions. Similarly, the deficit of receptors in these areas and also the mesen- 
cephalic reticular formation and posterior diencephalon would not lead one to expect a primary influence of cannabinoids upon the major CNS arousal systems that arise there.

\subsection{Effects of Cannabis Use}

The cannabinoids comprise a unique pharmacological class of compounds producing a multiplicity of effects. They produce mixed stimulation and depression of CNS activity in different areas and therefore partially mimic the activities of other centrally acting drugs, including stimulants, sedatives, tranquilizers, and hallucinogens. However, the degree to which cannabinoids' effects resemble those of any other single class of psychoactive drugs is too low to allow their joint classification (Consroe et al., 1976; Razdan, 1986). In addition, THC has also been wrongly classified as a narcotic and should be viewed as distinct from the opiates in this respect (National Commission on Marihuana and Drug Abuse, 1972).

\subsubsection{Acute Effects}

THC is rapidly absorbed during cannabis smoking, and its acute effects appear shortly thereafter; i.e. within 15 minutes with peak effects occurring between 30 and 60 minutes. Acute subjective effects are dose-dependent and generally last for about two to four hours. Nonetheless, performance decrements may persist for several hours after the feeling of intoxication has passed (Barnett et al., 1985). Intravenous (i.v.) injection of THC produces a similar profile of effects as after smoking (Ohlsson et al., 1980; Lindgren et al., 1981). When cannabis is ingested, THC's onset of action is some hours delayed and subjective effects last for 5-12 hours without a clear peak, which is consistent with the drug's pharmacokinetics. It is still uncertain whether THC adversely affects performance during the day after cannabis smoking. Some studies provided evidence for a 'hangover' syndrome (Yesavage et al., 1985; Heishman et al., 1990; Leirer et al., 1991), but others demonstrating acute performance impairment failed to reveal the existence of clinically significant residual effects the following day (Rafaelsen et al., 1973a, 1973b; Barnett et al., 1985; Leirer et al., 1989; Chait et al., 1985; Chait, 1990).

An early clinical account of cannabis intoxication was given by Bromberg (1934), a psychiatrist, on the basis of his own experience and observations of, and discussions with, other users.

"The intoxication is initiated by a period of anxiety within 10 to 30 minutes after smoking, in which the user sometimes ... develops fears of death and anxieties of vague nature associated with restlessness and hyperactivity. Within a few minutes he begins to feel more calm and soon develops definite 
euphoria; he becomes talkative ... is elated, exhilarated ... begins to have ... an astounding feeling of lightness of the limbs and body ... laughs uncontrollably and explosively ... without at times the slightest provocation ... has the impression that his conversation is witty, brilliant ... The rapid flow of ideas gives the impression of brilliance of thought and observation ... [but] confusion appears on trying to remember what was thought ... he may begin to see visual hallucinations. . flashes of light or amorphous forms of vivid colors which evolve and develop into geometric figures, shapes, human faces, and pictures of great complexity ... After a longer or shorter time, lasting up to two hours, the smoker becomes drowsy, falls into a dreamless sleep and awakens with no physiologic after-effects and with a clear memory of what happened during the intoxication" (Bromberg, quoted by Grinspoon and Bakalar, 1992).

Grinspoon and Bakalar considered Bromberg's account as a composite, exaggerated and overinclusive description of cannabis 'highs'. Nevertheless, it clearly demonstrates how diverse the subjective effects of cannabis can be. After low doses of THC, the effects are subtle and of short duration, and intoxication is generally undetectable to the observer. After high doses, intoxication may still be hardly noticeable but the most reliably produced physiological signs include reddening of conjunctivae due to dilatation of blood vessels and increased heart rate with a concomitant peripheral vasodilation (e.g. Weil et al., 1968; Benowitz. and Jones, 1981; Hollister et al., 1981; Maykut, 1985). Blood pressure slightly increases in the supine position but decreases upon standing, which may result in orthostatic hypotension and syncope in some individuals (Maykut, 1985). THC exaggerates task-elicited tachycardia as well as mean arterial blood pressure, suggesting that it may increase cardiovascular responsivity (Capriotti et al., 1988). Respiratory depression, bronchodilation, and decreases in intraocular pressure, salivary flow, skin but not oral temperature, grip strength, and REM sleep have also been observed (Hollister, 1971a, 1986; Jones et al., 1981). Despite anecdotal reports, pupil size is not affected by the drug (Weil et al., 1968), Neither are the electroencephalogram and deep tendon reflexes (Dewey, 1986; Hollister, 1986). Intravenous administration of THC produces variable changes in total cerebral glucose metabolism but consistently increases cerebellar metabolism (Volkow et al., 1991). Mathew and associates showed that cerebral blood flow decreases in all brain regions in inexperienced smokers but increases in some brain areas, particularly the frontal lobe, in experienced smokers (Mathew et al., 1989, 1993).

The principal psychological effect of cannabis is the 'high' which is sometimes a dream-like, euphoric state. The user's mood may vary from exhilaration to quiet introspection. The euphoric state is sometimes accompanied by mild state anxiety, tension, anger, and confusion (Mathew et al., 1993). Time perception is consistently changed. Time seems to slow down so that minutes may seem like hours (Hollister and Gillespie, 1970; Bech et al., 1973; Borg et al., 1975; Hicks et al., 1984). Temporal disintegration is associated to the altered time sense as 
another sign of cannabis intoxication (Melges et al., 1970a; Mathew et al., 1993). The latter was defined by Melges et al. as the difficulty to retain, coordinate, and serially index those memories, perceptions, and expectations that are relevant to the goal one is pursuing. The user has difficulty concentrating and thinking is troubled by the intrusion of thoughts with more unusual associations, which may result in disorganized speech. Both studies showed further depersonalization, i.e. an alteration in the perception or experience of the self whereby the usual sense of one's own reality is temporarily lost or changed (DSM-III-R). Depersonalization can be prominent, particularly after consuming high doses of THC. Users can become detached observers of their own intoxication, possibly explaining why the more experienced may appear normal in public. Depersonalization caused by other factors is often said to be a cognitive defense against dysphoric mood states such as anxiety and depression. However, many users react to the combined experience of temporal disintegration and depersonalization - symptoms that are also produced by psychotomimetics - with euphoria. Mathew et al. (1993) therefore concluded that it is unlikely cannabis-induced depersonalization serves this function. Furthermore, they and many others have demonstrated that all of the above-mentioned aberrations are transient: normal self-perception returns within a few hours after smoking.

As cannabis generally intensifies a user's prevailing mood, dysphoric reactions may also occur. Some individuals, particularly naive users or more experienced users consuming an unexpectedly high dose, may exhibit anxiety which is sometimes accompanied by paranoid thoughts. Anxiety may become so severe as to induce panic reactions, probably due to the user's fear to lose control of thinking and actions, and that these effects might never wear off. Due to a distorted perception of the body, the anxious user may think he is becoming insane or undergoing a life-threatening physical catastrophe. Although relatively uncommon, such panic reactions are probably the most frequent adverse reaction to moderate cannabis use (Grinspoon and Bakalar). If the user has some degree of underlying depression, cannabis may produce an acute depressive episode. This reaction is, however, rarely seen in experienced users. Another infrequently reported adverse reaction is the occurrence of a psychotic syndrome, described by Chopra and Smith (1974) as lying on a continuum from "an acute confusional state" to "a full-blown toxic psychosis." This acute organic psychosis is more likely to occur after consumption of an extremely high cannabis dose, particularly in individuals with a previous history of psychosis (Chaudry et al., 1991; Mathers and Ghodse, 1992). The syndrome resembles the delirium of high fever and includes disorientation, confusion, and both auditory and visual hallucinations (Weil, 1970; Maykut, 1985; Wert and Raulin, 1986). It can be treated effectively with cannabis withdrawal or antipsychotic drug administration, without residual effects (Chaudhury et al., 1989; Chaudry et al., 1991; Van Brussel, 1993). Cannabis may precipitate schizophreniform episodes (Thornicroft, 
1990) but, as the drug may exacerbate pre-existing mental illness, these are usually relapses of known cases (Tunving, 1985; Hollister, 1988).

Disruption of memory processes is the single most consistently reported cognitive deficit following cannabis use. Marijuana smoking impaired immediate free recall of digits (Tinklenberg et al., 1970; Heishman et al., 1989, 1990), words (Abel, 1971; Chait et al., 1985), prose (Miller et al., 1977a), and picture/word combinations (Miller et al., 1977b). Hooker and Jones (1987) found an increased number of short story omissions following marijuana smoking, and intrusions occurred in delayed free recall. However, neither immediate and sustained attention nor controlled retrieval from semantic memory were affected. Darley et al. (1974) found that subjects treated with drug and placebo did not differ with respect to either their delayed recall or delayed recognition of word lists that were learned four days earlier. This means that THC impairs acquisition or consolidation but not retrieval processes.

The recent breakthrough in localizing the sites of THC activity within the brain, coupled with earlier psychological concepts of the relationship between working memory and the temporal organization of behavior, may offer an explanation for the most prominent cognitive deficit in THC intoxication. Working memory is conceived as the brain's limited-capacity 'work space' for temporary storage and processing of sensory information in relation to that retrieved from long-term memory (Baddely and Hitch, 1974). Melges et al. (1970b) demonstrated that working memory impairment is at least partially responsible for temporal disorganization of behavior: errors in their GoalDirected Serial Alternation Task were mainly due to serially organizing and retaining information arriving in working memory. This finding suggests a crucial locus within the CNS which accepts incoming information, serially encodes it with respect to time of arrival and holds it long enough to form associations with information elicited from long-term memory. Abundant evidence from neuropsychological research indicates the hippocampus is that crucial coordinating structure (Gray, 1982). The localization of a high density of cannabinoid receptors within the hippocampus encourages one to speculate concerning the drug's probably related amnestic and temporally disorientating properties. As mentioned above, the disruption of organized consciousness by the intrusion of irrelevant thoughts is quite common following cannabis use. Since the hippocampus functions to inhibit the inappropriate recall of long-term memory associations as well as facilitating the recall of appropriate ones (Valzelli, 1980), it is tempting to speculate that cannabinoid receptor agonists, including THC, reduce the internal inhibition of associations. The intrusion of inappropriate associations in working memory would result in impaired learning (Hooker and Jones, 1987).

THC can have other disinhibiting effects on information processing. The Stroop Color-Word Test was specifically designed to measure a subject's ability 
to inhibit a stereotyped, but under the circumstances, inappropriate response. In this test, the names of colors are rapidly presented in compatible or incompatible colors. An example of the latter would be the presentation of the word 'red' in a blue color. The subject has to inhibit the stereotyped response of reading ('red'), and instead, specify the color of the characters (blue). Disinhibitory drugs cause the tendency to revert more often to the stereotyped response. THC is disinhibitory: Hooker and Jones found that THC produced significantly more erroneous stereotyped responses, while independent measures of word reading and color naming speed were not affected.

Besides affecting memory processes and time estimation, several reviews demonstrated that THC produces performance decrements in many different tests of perceptual, cognitive, and motor skills, such as signal detection, attention, motor coordination, and reaction speed (Moskowitz, 1985; Murray, 1985; Chesher, 1986). The effects of THC on skills important for driving, as measured in the laboratory and in driving simulators, are more extensively discussed in the next chapter.

Many users of cannabis claim that the drug heightens their sensitivity to external stimuli, sharpens their vision (though with many visual distortions), makes colors appear brighter, enhances the appreciation of music, and reveals details that are normally neglected. In the words of Grinspoon and Bakalar "It is as though the cannabis-intoxicated adult perceives the world with some of the newness, wonder, curiosity, and excitement of a child (p. 237)." Possibly these are the positive aspects of perceptual disinhibition, allowing the formation of associations that have been long since relegated to the 'irrelevant' by the process of habituation. As a result, some people believe that they become more creative and perform better while under the influence of the drug. Notwithstanding the sincerity of their claims, enhanced creativity under the influence of THC has never been demonstrated in a manner that would withstand scientific scrutiny.

Another argument advanced by THC advocates is that the drug increases appetite (Tart, 1970). This assertion was repeatedly investigated and confirmed in laboratory settings, i.e. acute administration of marijuana selectively increased food consumption (Weil et al., 1968; Hollister, 1971b; Foltin et al., 1986, 1988; Kelly et al., 1990). Weil and his colleagues measured but failed to find concomitant changes in blood glucose levels and therefore postulated a central rather than peripheral physiological trigger for increased appetite. Foltin and his colleagues found that the total increase in caloric intake on days that subjects smoked marijuana was not attributable to eating during regularly scheduled meals but rather to ad lib eating between meals. Apparently, THC has less effect upon mechanisms of hunger and satiety than it does upon those that determine the positive hedonic value of certain foods. As a result of increased food intake, body weight increases also but to a greater extent than predictable on the basis of caloric intake alone (Foltin et al., 1988). 
Cannabis is sometimes referred to as an hallucinogen. Many of its perceptual and cognitive effects are also produced by lysergic acid diethylamide (LSD), including depersonalization, temporal disintegration, and distortions in color vision. Furthermore, both drugs may incidentally produce anxiety and paranoid reactions. But, according to Grinspoon and Bakalar, there are also important differences between subjective effects of the two drugs. LSD's are far less controllable by the individual. The so-called 'bad trip' - an experience of agonizingly nightmarish reactions - is far more common among LSD users, even experienced ones, than among cannabis users. But there is a more important difference between cannabis and hallucinogens. Except after very high doses, whatever the cannabis user subjectively experiences as a unique drug effect is superimposed on the normal stream of consciousness. What the LSD user experiences sometimes replaces it. Grinspoon and Bakalar therefore concluded that it is unlikely that cannabis, when consumed in normal doses, will produce true hallucinations. After very high doses of marijuana, however, hallucinogenic trips that approach those of LSD may occur (Tart, 1970; Fabian and Fishkin, 1981).

Cannabis has been used as a stimulant for work and a depressant for relaxation (National Commission on Marihuana and Drug Abuse, 1972; Murray, 1985; Van Ree and Essenveld, 1985). It is sometimes said that the drug-induced euphoric state is followed by drowsiness (Maykut, 1985; Hollister, 1986). But this opinion seems questionable from the failure to locate dense concentrations of THC receptors within the brain's major arousal generators, mentioned above, and the lack of consistent EEG signs of diminished electrocortical arousal following acute drug administration (Dewey, 1986). Chait et al. (1985) found no THC effect on reported quality of sleep, sleep onset latency, or number of awakenings during the night after marijuana smoking. In a similar study, but now using multiple doses over several consecutive days, Chait (1990) found a significant effect on only one of the four factors measured by the Leeds Sleep Evaluation Questionnaire: "getting to sleep" was easier after continual marijuana than placebo smoking. This effect seems more attributable to increased relaxation than sedation. Jones (1971) showed that the occurrence of drowsiness may be contingent upon the social setting: when tested individually, subjects demonstrated slight drowsiness but, when tested in a group situation, there was a marked lack of sedation. Furthermore, Jones noted that symptoms indicative of drowsiness, such as feeling sluggish or sleepy, were more often reported in the placebo than active drug condition. Thus, there is no convincing evidence that THC itself produces pronounced sedation. 


\subsubsection{Factors Influencing the Drug's Acute Effects}

The wide range of different individuals' reactions after cannabis smoking is well known. But the same individual can also react inconsistently after smoking on different occasions. Several factors responsible for inter- and intrasubject variability have been identified. They include the route of administration, the THC dose, the smoking technique, the drug's potency, the individual's previous drug experience, and the psychological set and physical or social setting.

It is obvious that the THC dose and route of administration are important factors in determining the timing and magnitude of acute effects. As mentioned above, increasing THC doses not only increase all acute effects but may eventually lead to qualitatively different reactions. Despite every effort to control the dose via a chosen route of administration, it is usually quite difficult to ensure that the quantity of THC entering the body, much less the brain, will be the same in different individuals or the same tested repeatedly. Nearly perfect control over the administered amount of THC is achieved when it is injected intravenously. This route of administration has, however, some important disadvantages (Dewey, 1986). First, the cannabinoids are very lipid soluble and posses hydrophobic properties. They are difficult to handle because of their high viscosity, illustrated by Dewey's term "rubber cement", making it difficult to accurately prepare small doses. Secondly, different vehicles have been used to put THC into solution, including ethanol and many others. Since the vehicle might interact with THC in different ways, discrepancies in effects can easily appear when different vehicles are used. Finally, and due to these difficulties, cannabis users do not inject their drug. Using this route of administration in experimental studies with humans may therefore lead to results that are not representative of the naturally occurring events.

Administering THC by the oral route provides good control of the administered dose but much less over the amount of THC entering the systemic circulation, due to the great interindividual variability in rate of absorption from the digestive tract and first-pass metabolism in gut and liver. Another disadvantage of oral dosing is the difficulty it imposes on titrating the plasma/brain concentration to achieve the desired effect: underdosing with no effect and overdosing with adverse reactions would be the norm rather than the exceptions. But the most important disadvantage is again that it is not the route of drug administration preferred by the majority of cannabis users. Results obtained using this approach are unlikely to be representative of THC's effects in real life.

Cannabis smoking is generally associated with a considerably different smoking topography than that observed during tobacco smoking, including larger puff volumes and somewhat larger inhalation volumes (Wu et al., 1988). Smokers of cannabis also tend to hold the smoke in their lungs for longer periods of time, typically 10 to 15 seconds, owing to their belief that prolonged breathholding 
increases THC absorption and, consequentially, the subjective effects (Perez-Reyes et al., 1981, 1982; Wu et al., 1988). Zacny and Chait (1989) measured the psychological and physiological effects of THC under three different breathholding conditions, 0,10 and 20 seconds, while maintaining the inhaled volumes of smoke and ambient air constant. Though the 0 -second condition implied that smoke should be immediately exhaled after inhalation, the actual duration that smoke was in the lungs was estimated by the investigators as probably longer than five seconds. Compared to baseline, THC produced significant elevations on the primary measures - perceived 'high', heart rate and expired air carbon monoxide (CO) - but the effects were not significantly related to breathhold duration. The study was replicated by the same investigators but now with the addition of a placebo condition and the omission of the 10-second breathholding condition (Zacny and Chait, 1991). Compared to placebo, THC increased heart rate and subjective effects. Prolonged breathholding magnified the heart rate elevations moderately (about $20 \%$ ), albeit with borderline statistical significance, but had no effect on subjective measures. In this study, expired air co did increase with prolonged breathholding but to a similar extent in both the placebo and active drug conditions, indicating that THC does not affect alveolar absorption of CO. Tashkin et al. (1991) executed a similar study, but they also measured drug plasma concentrations. They examined the effects of breathhold duration ( 4 or 14 seconds), puff volume ( 70 or $45 \mathrm{ml}$ ), and number of puffs (6 or $10)$ on the amount of inhaled tar, tar retention in the lung, blood carboxyhemoglobin, plasma THC concentration, heart rate, and subjective 'high'. Changes in puff volume and number of puffs had no effect on any of these measures if cumulative puff volume was held constant. Prolonged breathholding had no effect on the amount of inhaled tar, but did produce greater retention of tar in the lungs and greater blood carboxyhemoglobin concentrations. Furthermore, it produced significantly higher plasma concentrations of THC two minutes after completion of smoking, accompanied by somewhat greater elevations in heart rate and slight increases in subjective 'high'. Block et al. (1992) measured the acute effects of smoking marijuana cigarettes containing $19 \mathrm{mg}$ THC, and placebo, on a wide variety of cognitive and psychomotor tests. They also examined the effects of breathhold duration, i.e. 7 and 15 seconds. As the latter was only a secondary goal of their investigation, they failed to assess other parameters of the subjects' smoking behavior. Nor did they measure heart rate, plasma concentrations of THC, or $\mathrm{CO}$ absorption. The results showed significant performance decrements in most tests following THC. Subjects' performance was worse after long than short breathhold duration, but this was independent of the material smoked. In other words, prolonged breathhold duration did not potentiate THC's adverse effects. Together, these studies show that prolonged breathholding produces increased absorption of THC, somewhat greater tachycardia, but only 
slightly greater subjective effects and hardly any larger performance deficits. Thus, it seems unnecessary to control breathholding time in experimental studies.

As mentioned earlier, marijuana's THC concentration, or potency, is increasing rapidly. It is therefore important to examine whether marijuana smokers modify their smoking behavior depending on the potency of the drug, like tobacco smokers do depending on a cigarette's nicotine yield (Herning et al., 1981; Gust and Pickens, 1982). If individual cannabis smokers always titrate plasma/brain concentrations to the same levels, the magnitude of effects produced by different potencies should be the same in any given one. Yet there are reasons to believe that this is not the case. Heavy users showed pronounced subjective effects to placebo cigarettes in Jones' study (below) and several others failed to reveal significant changes in smoking patterns as a function of THC concentration. Cappell et al. (1973) allowed subjects to smoke as many marijuana cigarettes, containing either $0.2,0.4$, or $0.8 \% \mathrm{THC}$, as they needed to achieve a 'nice high'. The estimated weight of marijuana consumed was inversely related to drug potency but the 4-fold increase from lowest to highest potency yielded only a $27 \%$ decrease in marijuana consumption. Puff duration and interpuff interval were nearly identical in the three conditions but cumulative breathhold duration and number of puffs were inversely related to potency. Though consistent with a titration process, the latter results were not significant. Similar results were achieved when the study was replicated with potencies of $0.36,0.73$, and $1.45 \%$ (Cappell and Pliner, 1974). Perez-Reyes et al. (1982) observed smoking patterns when experienced smokers consumed marijuana cigarettes containing 1.32, 1.97, and $2.54 \%$ THC but also failed to show significant changes as a function of potency. These subjects inhaled more THC with higher potencies resulting in higher plasma levels of cannabinoids as well as greater psychological and physiological reactions.

In these studies, the investigators measured puff duration and number of puffs but failed to obtain volumetric measures. Herning et al. (1986) did while heavy smokers consumed marijuana cigarettes containing 1.2 and $3.9 \%$ THC. Their interpuff interval and number of puffs increased while smoking the high potency cigarette, resulting in a prolonged smoking period. They did not alter puff duration but inhaled a larger volume of air, thereby diluting the marijuana smoke. Although these smokers adjusted their smoking pattern they still did not titrate THC because the cumulative puff volume of the high potency cigarette was twice that of the low potency. Chait (1989) supposed that the failure of previous studies to demonstrate true titration might be attributable to the subjects' unfamiliarity with the different potencies presented in the cigarettes given by the investigators. In his study, subjects were given the opportunity to experience the respective potencies $(0.9,1.7$, and $2.7 \%)$ of each color coded marijuana type as they smoked them on five separate occasions. In all cases these subjects were allowed to self-administer as much marijuana as they wanted over a 30 -min 
period. Yet the subjects' THC consumption, as measured by several reactions, increased as a function of cigarette potency. They did not regulate the dose in spite of their knowledge of the different potencies.

In contrast to previous results, subjects in a study by Heishman et al. (1989) appeared to adjust their smoking pattern depending on the marijuana potency. The high potency cigarette ( $2.7 \%$ THC) was smoked with a shorter puff duration and a lower puff and inhalation volume than the low potency cigarette $(1.3 \%$ THC). Interestingly, smoking patterns began to diverge after the second or third puff. Whether the subjects' ability to adjust their smoking pattern with higher potency cigarettes was due to pharmacological factors is, however, doubtful. They showed it more unpleasant to smoke the high potency cigarettes owing to 'harshness', which may have strongly contributed to the observed differences. In conclusion, there is only weak evidence that marijuana users adjust their doses of inhaled THC so as to titrate plasma/brain concentrations to a desired level. But before closing the issue, it should be recognized that the range of potencies used in experimental studies was always narrow. The smokers may have been unable to discriminate among them and titrate successfully. Users in real life are not only confronted with a wider range of potencies. They are usually more aware of the potency of the material they smoke, from prior experience, upon the advice of other users or even from its 'street' price. It might well be that ordinary users regulate the consumed dose more accurately than shown by these studies.

In many studies in which THC was administered by smoking, subjects were allowed to smoke the cigarettes ad lib, i.e. in their customary fashion and as much of the administered dose as they wished. This was done to keep the experimental setting as natural as possible. Those investigators who applied strict smoking procedures, with or without the use of special devices, did so in order to exert greater control on the amount of THC inhaled by the subjects. Besides being less natural and probably also annoying, the latter procedure may result in large numbers of subjects reporting discomfort and dysphoric reactions (Mathew et al., 1989). The major drawback of the ad lib procedure is, of course, that one is less certain of how much THC was actually delivered. Cumulative puff volume may be used to estimate the inhaled dose, but measuring it is probably as troublesome to the subjects as the rigidly structured smoking procedures. Direct comparisons between the observed effects of THC in different studies can only be reasonably made if investigators measure and report at least the administered dose, but preferably also drug plasma concentrations. The total amount of smoked THC can be estimated gravimetrically by multiplying the potency of the drug by the weight of the smoked portion. This is a legitimate procedure: it has been shown that the THC concentrations in the unsmoked portions of marijuana cigarettes are very similar to those found in the unlit cigarette (Perez-Reyes et al., 1982). One is tempted to conclude from the results reported in the literature that 
the different smoking procedures do not produce disparate results. The main reason is that THC concentrations in plasma show great interindividual variability whether an ad lib or paced smoking protocol was used. Apparently, individual differences in absorption, distribution, and metabolism of THC have a greater impact on the observed plasma concentrations than the smoking procedure. Yet the two procedures have never been directly compared in a single study. A conclusive answer concerning the differential effects of smoking procedures on the delivery of THC or its effects can not be provided for that reason.

A factor that has probably a much greater impact on the severity of intoxication and the extent of performance deficits than the smoking technique is the individual's previous cannabis experience. While light and heavy users - light meaning usually not more than once a month, and, heavy, at least once daily reported similar subjective 'highs' following THC administration by either smoking or i.v. injection (Jones, 1971; Perez-Reyes et al., 1974; Lindgren et al., 1981), nonusers experienced significantly less effects than users (Weil et al., 1968; Casswell and Marks, 1973; Milstein et al., 1975). These observations agree with anecdotal reports of chronic users that a novice user has to 'learn to get high'. Weil and his colleagues advanced two alternative interpretations of this phenomenon. The first is that 'reverse tolerance' or some sort of pharmacological sensitization occurs after repeated exposure to cannabis, but no study has provided evidence for this hypothesis (Hollister, 1986). Another possibility hypothesized by Weil et al. is that novice users are psychologically inhibited from experiencing any effect, and that the inhibition is reduced after repeated exposure to cannabis. A more likely explanation, though related to the latter, is that users and nonusers have different expectancies that modify their reactions. This possibility is discussed below.

Whereas subjective measures of intoxication showed consistently greater effects in users than nonusers, objective tests of cognitive and psychomotor performance have provided inconsistent results. Experienced users exhibited similar impairments in cognitive functioning (Casswell and Marks, 1973), greater impairments in perceptual-motor tasks (Milstein et al., 1975), and less impairment in a divided attention task (Marks and MacAvoy, 1989). Compared to light or casual users, however, heavy users showed consistently less impairment in digit symbol substitution, complex reaction time, and continuous performance tests following active drug administration (Jones, 1971; Meyer et al., 1971). These observations seem to confirm the conviction of many experienced marijuana users that they can volitionally control THC's effects (Tart, 1970). The notion is supported by the common observation that the total effects of marijuana smoking contain a large placebo component (below). It was experimentally tested by Cappell and Pliner (1973). Twenty subjects smoked a marijuana cigarette containing $12 \mathrm{mg}$ THC or placebo on two separate occasions. Before smoking, baseline measurements were taken of heart rate and performance in four tasks, 
i.e. time estimation, immediate recall of words, solving arithmetic problems, and backward digit span. After smoking, the subjects rated their level of intoxication. At this point, the major experimental manipulation was introduced. Unbeknown to them, the subjects were equally divided between two groups matched on the basis of self-reported current marijuana use. The low-motivation group was simply required to repeat the same tasks. The high-motivation group received the instruction to repeat the tasks while trying as hard as possible to overcome the drug's interfering effects. After they were over, both groups again rated their intoxication. Instructions had no effect on heart rate or feelings of intoxication. Arithmetic and backward digit span were unaffected by THC and so showed no effects of instructions. Immediate recall and time estimation were impaired in the low-motivation group and memory was likewise impaired in the others. However, the high-motivation group showed significantly less drug effect on time estimation. This result shows that some volitional control of THC's effects is possible. It also indicates that the reasons large THC effects on performance have been found in some experiments but not others is not only related to differences in the investigators' testing procedures but also to their respective subjects' motivation for overcoming those effects by compensatory effort.

As already mentioned, the high variability of cannabis' effects between individuals may be partially attributable to differences in the expectations of the user. Jones (1971), for example, found that ratings of psychological 'high' were greater in heavy than light users following placebo but similar following the active drug. This suggests that heavy users tended more to respond to cues as smell and taste which were similar for both placebo and active marijuana. This interpretation was further strengthened by the lack of a subjective effect in both heavy and light users following the oral ingestion of placebo marijuana that had a disagreeable taste. Camí et al. (1991) also showed that expectancy may influence smoking behavior. Subjects who expected and received active drug (hashish incorporated into a tobacco cigarette) showed higher plasma levels of both THC and $\mathrm{THC}-\mathrm{COOH}$ as well as faster metabolism than subjects who had not expected but received active drug. Furthermore, the elevation in heart rate was significantly greater in the former group who also experienced more marked subjective effects. Interestingly, another group of subjects who expected active drug but received placebo, showed pronounced subjective effects. A similarly strong placebo effect was found by Chait and Perry (1992). Their regular marijuana smokers were allowed to smoke placebo cigarettes freely for 60 minutes during four identical weekly sessions. Some were told that the cigarettes contained THC (deceptive administration), and others, that they might or might not (doubleblind administration). The deceived subjects smoked more placebo marijuana and reported a greater subjective response than the others, though the difference was confined to the first session. Together, these studies show that subjects' expectancies can have a significant impact on perceived THC effects. Moreover, they 
clearly demonstrate the need for inclusion of placebo marijuana (or hashish) in experimental research.

Another factor deemed important in determining the effects of cannabis is the setting, i.e. the prevailing environmental and social conditions during drug use. Hollister et al. (1975) investigated the effects of two settings, one 'favorable' and the other 'neutral', on the psychological and physiological effects during marijuana smoking. Twelve subjects were tested in both settings, once after smoking placebo and once after active marijuana. There was a clear effect of active drug along with substantial variability between subjects' reactions, but the actual setting in which the drug was administered did not significantly alter those effects. One's company during marijuana smoking seems a more powerful mitigating factor than the physical environment. As mentioned above, Jones found substantial differences in reactions to marijuana smoking between subjects tested individually and those tested in a group. The former exhibited the relaxed, slightly drowsy, and undramatic state typically seen in laboratory settings, whereas the latter showed elation, euphoria, uncontrolled laughter, and a marked lack of sedation. Further studies demonstrated that an individual's mood after marijuana smoking is significantly related to the prevailing moods of the group (Rossi et al., 1978); and, that THC produces greater perceived 'highs' when smoked in the presence of friends than strangers (Marks and Pow, 1989).

In conclusion, many factors may influence THC's effects but only a few seem to really matter. The physical setting and smoking technique appeared to only slightly influence THC's effects whereas the social setting and the user's expectations based on previous cannabis experience influenced them substantially. Potent psychosocial factors, more than differences in the relatively low to moderate THC doses that are typically consumed for recreational purposes seem to determine how THC affects behavior in real life situations. Or, as Jones concluded more than two decades ago, "... many people have uncritically accepted the belief that the drug has specific effects on behavior and experience and that these can be readily identified... Although at high doses such a model may be valid, at the doses most youthful drug users are discussing there is ample evidence that the effects of psychoactive drugs on behavior and experience are often to a great extent independent of the drugs' pharmacological effects (p. 368)." Regarding the design of experimental research on cannabis, these observations strongly imply the need for placebo-controlled, double-blind, repeatedmeasures designs, in which each subject acts as his or her own control; and, to maintain the constancy of psychosocial factors, when the design is to measure THC's pharmacological effects, or systematically vary them for measuring the mitigating effects. 


\subsubsection{Chronic Effects}

Disapproval of cannabis stems from a concern about the alleged consequences of its use, particularly over a prolonged period of time. It has been claimed that cannabis use leads to antisocial and criminal behavior, is a stepping stone to dependence on heroin and other more harmful drugs, and produces psychosis, 'amotivational syndrome', and many physical disorders (Davison and Neale, 1978). The scientific findings regarding the asserted sequelae of long-term cannabis use are summarized in this section.

Whereas the acute effects of cannabis are relatively well established, consensus concerning the consequences of long-term use is lacking among researchers. This is particularly true regarding THC's supposed psychopathological effects. The major reason is that previous epidemiological and field studies have been unsuitable for determining the cause-effect relationship. Cross-sectional comparisons of users and nonusers fail to reveal whether drug use is the cause of psychopathology or whether both are the consequence of the same underlying problem. Although cannabis is most commonly taken for pleasure, the drug is also used as a facet of adolescent experimentation, to demonstrate independence or rebelliousness, cope with anxiety, or as 'self-medication' for early symptoms of mental illnesses such as depression or schizophrenia (Grinspoon and Bakalar, 1992). Prudence is therefore warranted when attributing a psychopathological or physical syndrome to chronic cannabis use when only an association between the two has been proven.

The cause-effect problem can be clearly illustrated by a most important longitudinal investigation of the relation between psychological characteristics and drug use (Shedler and Block, 1990). The investigators followed 101 San Francisco children from ages 3 to 18 , and assessed many psychological measures at ages $3,4,5,7,11,14$, and 18 . The parent-child interaction was measured at age 5. Information regarding their drug use was collected at age 18 . By then $68 \%$ had used marijuana at least once and $39 \%$ were currently using it at least once a month, $21 \%$, at least once a week. Large minorities had also used other drugs. Most of the sample could be divided into three groups: abstainers (29), experimenters (36), and frequent users (20). Sixteen did not fit into any category and were largely ignored in the authors' conclusions. The identifiable groups did not differ in socioeconomic status or IQ. The picture of frequent users that emerged was "one of a troubled adolescent, an adolescent who is interpersonally alienated, emotionally withdrawn, and manifestly unhappy, and who expresses his or her maladjustment through undercontrolled, overtly antisocial behavior." Abstainers were relatively "tense, overcontrolled, emotionally constricted ... socially isolated and lacking in interpersonal skills," and experimenters appeared as the best-adjusted in the sample (The investigators hastened to add that these findings should not be misinterpreted as indicating that drug use might somehow 
improve an adolescent's psychological health). Solely on the basis of these observations one might conclude that frequent cannabis use leads to personal and social maladjustment. Yet the psychological differences between frequent drug users, experimenters, and abstainers could be traced to the earliest years of childhood and related to the quality of parent-child interactions. At seven and eleven years of age, future frequent users got along poorly with other children, were insecure, and showed numerous signs of emotional distress. Abstainers, at the same ages, were relatively overcontrolled, shy, fearful, and morose. At five years of age, mothers of both abstainers and frequent users were relatively cold and unresponsive. While pressuring their children to perform well, they gave them little encouragement. Fathers of frequent users were not different from those of experimenters, but fathers of abstainers were impatient, domineering, and squelched spontaneity and creativity. The investigators concluded from their observations that "(a) problem drug use is a symptom, not a cause, of personal and social maladjustment, and (b) the meaning of drug use can be understood only in the context of an individual's personality structure and developmental history." Of course, these findings should be replicated before final conclusions are drawn but they highlight a very important point that is too easily overlooked, i.e. simple associations between phenomena are never conclusive evidence for alleged cause-effect relationships.

Early reports emanating from law enforcement authorities and the story of the bashshashim (Section 2.1) led to another questionable association, i.e. that between cannabis use and violent and criminal behavior. It appears true that heavy cannabis use was more prevalent among criminals, before the 1960 s when it was mainly confined to dissident minorities, but it does not necessarily follow that cannabis was the cause of their criminal acts. Though symptoms of bhanginduced toxic psychosis may include hostile feelings (Chaudry et al., 1991), cannabis failed to increase hostility in experimental studies involving healthy, occasional cannabis users (Salzman et al., 1976; Marks and Pow, 1989). Abel (1977) reviewed the relationship between cannabis use and violence, and concluded that cannabis does not precipitate violence in the vast majority of users though it may in unstable individuals. The consensus today is that, if anything, cannabis seems to inhibit violent and aggressive behavior (National Commission on Marihuana and Drug Abuse, 1972; Hollister, 1986; Grinspoon and Bakalar, 1992).

Another association that has been wrongly interpreted as showing a causal relationship is between the use of cannabis and other, more harmful, drugs such as heroin and cocaine. Some assume that the former is a stepping stone to the latter. While it is true that many heroin and cocaine addicts began their illicit drug 'career' with cannabis, the majority of cannabis users do not take these other drugs (Davison and Neale, 1978; Grinspoon and Bakalar, 1992). Similarly, most cannabis users first took licit drugs such as alcohol and nicotine by smoking 
tobacco (Ellickson et al., 1992), but the majority of alcohol and tobacco users do not smoke cannabis. Thus, if cannabis can be considered a stepping stone to more harmful drugs, alcohol and tobacco would fulfil that function for cannabis. One deduction of the stepping stone theory is that fewer people would be addicted to opiates if cannabis were not available. There is, however, little evidence for this hypothesis. Although cannabis users are more likely to become involved with other illicit drugs than nonusers, it seems very unlikely that THC itself causes the craving for other drugs. A more likely explanation is that users of any given drug generally show greater interest in experimenting with other drugs. Furthermore, the common subcultural scene in which both cannabis and other illicit drugs are available from the same source makes it easier for users of the former to become involved with the latter (Johnson, 1973; Cohen, 1975).

One of the major reasons for many people's disapproval of cannabis has been the possible development of an 'amotivational syndrome'. This syndrome is characterized by a state of apathy and bluntness, along with a loss of interest in personal appearance and conventional goals. This personality disorder was manifest among frequent users as described by Shedler and Block (1990). As before, there is no reason to preclude a causal relationship. Neither is there a compelling reason to accept its existence. Many authorities have concluded that there is no convincing evidence that the amotivational syndrome is a direct consequence of marijuana use (e.g. Campbell, 1976; Hollister, 1986; Grinspoon and Bakalar, 1992).

Another alleged sequela of repeated cannabis use is the possible occurrence of 'flashbacks'. Flashbacks are spontaneous recurrences of a drug's effects, during a drug-free period, that are similar to those experienced earlier while under its influence. Their duration is usually much shorter, from seconds to minutes, but there are also reports of flashbacks that persisted for weeks or months and required psychiatric treatment (see review by Fischer and Täschner, 1991). The etiology of flashbacks is still unknown but it is well established that they can occur in hallucinogen users. Most inquiries into the incidence of flashbacks among LSD users have determined that about 30\% experience them at least once (Yager et al., 1983), but more extreme values in both directions have been reported: Fischer and Täschner (1991) surveyed the literature to show that incidence rates vary between 15 and $75 \%$. This wide variation is probably due to the fact that various investigators apply different definitions of flashbacks and usually rely on the users' recall of their occurrence. The same and other problems arise when attempting to define the occurrence and frequency of flashbacks in cannabis users. The fraction of cannabis users who report flashbacks are mainly the greatest consumers. As mentioned above, their subjective reactions to cannabis contain a large placebo component based on expectations. Similarly, they might selectively attend to experiences of naturally occurring altered physiological and psychological states and attribute them to a spontaneous 
recurrence of previous intoxication (Heaton and Victor, 1976). Another complicating factor is that the majority of cannabis users who report flashbacks also have previous experience with much stronger hallucinogenic drugs, such as LSD and mescaline (Tunving, 1985). And, as a rule, flashbacks may well occur in these cases. There are some reports showing that flashbacks occur in individuals whose sole illicit drug use is cannabis, but, according to Fischer and Täschner, they are unusual enough to warrant a thorough diagnostic evaluation in every case.

As mentioned in the previous section, cannabis may trigger immediate organic psychosis and precipitate schizophreniform episodes. Though evidence for chronic organic reactions is sparse (Thornicroft, 1990), chronic cannabis use is associated with an increased risk of developing schizophrenia in the subsequent fifteen years (Andréasson et al., 1987). It has, however, also been suggested that cannabis use might be more prevalent in people with greater pre-existing social and psychological vulnerability, and that chronic users might have become mentally ill in any case (Johnson et al., 1988; Negrete, 1989). Mathers and Ghodse (1992) compared 61 newly admitted in-patients with psychotic symptoms and cannabis-positive urine analysis to 43 control patients with psychotic symptoms but with drug-free urine analysis. Interestingly, a far greater proportion of cannabis-positive patients had histories of other drug use than the controls. Patients were interviewed within one week of admission and again at one and six months, using the 140-item Present State Examination. At the first assessment, the two groups differed on only five items: changed perception, thought insertion, nonverbal auditory hallucinations, delusions of control, and of grandiose ability. Although the number of discriminating items were no more than expected on the basis of chance alone, they are consistent with acute cannabis intoxication. Only one item (delayed sleep) discriminated between the groups at one month, and none at six months. The authors concluded that shortlived psychotic episodes can occur in clear consciousness after cannabis intoxication, but the development of chronic cannabis-induced psychosis is unlikely. They as well as Thornicroft (1990) discouraged psychiatrists' use of the diagnostic label 'cannabis psychosis' because it may delay the correct alternative diagnosis, usually paranoid schizophrenia. Thus, it seems unlikely that chronic use of cannabis can produce a functional psychosis de novo in a stable individual. But it is quite likely that cannabis provokes, exacerbates, or prolongs pre-existing mental disturbances (Tunving, 1985).

As mentioned above, working memory impairment is one of the most consistently reported acute effects of THC. This raises the logical question whether chronic use of cannabis is associated with similar, more persistent, impairments. Early studies conducted in Jamaica, Greece, Costa Rica, and India failed to reveal any significant impairment of cognitive functions in chronic cannabis users (Dornbush and Kokkevi, 1976; Stefanis et al., 1976; Coggins, 1976; Venkoba Rao et al., 1975), but one Egyptian study did (Soueif, 1975). Most of 
these studies, however, have been criticized for many different reasons including small sample sizes, inadequate sampling techniques, lack of test standardization, and acute intoxication (Hollister, 1986; Solowij et al., 1991). Mendhiratta et al. (1978) compared 25 charas smokers and 25 bhang drinkers to 25 nonusers of cannabis, and found that users scored worse on backward digit span, pencil tapping, time and size estimation, recognition and reproduction of designs, and were slower in producing associations to words. After a lapse of ten years, they re-evaluated $60 \%$ of the subjects (i.e. 30 users and 15 nonusers) and found similar impairments, except in recognition and size and time estimation, which were not measured (Mendhiratta et al., 1988). Varma et al. (1988) compared 26 long-term heavy users with 26 controls who were matched in terms of age, education, and occupation. A comprehensive battery of tests was applied to measure cognitive, perceptual-motor, and personality variables. Performance impairments of users relative to nonusers were similar to those previously found by Mendhiratta et al. Varma et al. also measured ten different memory functions but found a significant difference between the groups in only one; i.e. users had poorer recent memory than nonusers. They concluded that "the differences, if any, between users and nonusers in terms of cognitive functions pertain to perceptuo-motor tasks." Users were less capable in personal, social, and vocational functioning, and showed higher psychoticism and neuroticism, but their relative disabilities failed to reach clinically significant levels. Varma et al. found no differences between the groups with respect to intelligence but León-Carrión (1990) reported that 23 daily users of cannabis had lower scores on nine of the fourteen subscales of the Wechsler Adult Intelligence Scale than 24 controls coming from the same socioeconomic and cultural strata. Finally, Solowij et al. (1991) compared auditory reaction times and evoked, event-related EEG potentials of nine chronic cannabis users with nine nonusers who were matched on age, sex, and years of education. Subjects participated in four conditions in which they were instructed to attend to a particular location (left or right) and pitch (1047 or $1319 \mathrm{~Hz}$ ). Stimuli were either of short $(51 \mathrm{~ms})$ or long $(102 \mathrm{~ms})$ duration. Subjects were required to discriminate between them and respond to the latter. Users responded as rapidly as nonusers but with fewer correct and more false detections. The negative-going phase of early evoked potentials after short-duration stimuli was greatly enhanced in users relative to nonusers. The authors interpreted this as showing that the users engaged in superfluous pitch processing and were therefore less able to selectively attend to the duration of stimuli. These studies demonstrate that chronic use of cannabis may be associated with psychomotor and cognitive deficits, although, at the same time, deficits in chronic users seem to be less pervasive than those occurring during acute intoxication (Block et al., 1992). Yet none of the forementioned examined the possibility that users and nonusers differed on the evaluated variables, or other characteristics, prior to the 
former group's experience with cannabis. Prospective studies are still required for establishing the causal role of cannabis in producing these deficits.

It appears unlikely that gross neurological abnormalities develop as a result of chronic cannabis use. Cerebral blood flow is lower in chronic cannabis users, without significant regional blood flow abnormalities, and tends to return to normal with abstinence (Tunving et al., 1986). Chronic users' EEG patterns are similar to those from sedative addicts or normal drowsy individuals; i.e. all show increased alpha-activity and reduced beta-activity with a general slowing of activity across the EEG frequency band (Tunving, 1985). However, there are no signs of neurotoxicity, cerebral atrophy, or diminished cerebral function in chronic marihuana users (Farre-Albaladejo, 1989; Fehr and Kalant, 1983; Co et al., 1977).

The discovery of cannabinoid receptors in human leukocytes corroborates earlier observations that THC can impair immune responses by inhibiting Tlymphocyte proliferation and function, and suppressing $\gamma$-interferon production (Hollister, 1986; Bouaboula et al., 1993). While one is tempted to deduce from these findings that chronic cannabis users are more susceptible to opportunistic infections or malignancy, Hollister reported that the confirming clinical evidence is still lacking. Yet the potential immunosuppressive effect of THC is an important issue, even more so since the U.S. Food and Drug Administration (FDA) approved of dronabinol, a synthetic THC analog, for treatment of anorexia in AIDS patients (below). Drugs that are potentially immunosuppressive pose a grave risk to these patients. Dronabinol's net effect upon mortality in this population should therefore be carefully monitored.

Impaired pulmonary function is perhaps of greatest significance among the potential physical sequelae of long-term cannabis use. Heavy hashish use by soldiers has been associated with chronic bronchitis, chronic cough, and precancerous mucosal changes; i.e. squamous metaplasia (Henderson et al., 1972). Marijuana smoking may produce mild but significant airway obstruction (Tashkin et al., 1976). Compared with tobacco smoking, it delivers greater amounts of the carcinogenic products, tar and benzopyrene to the mouth and leads to greater retention of the inhaled tar in the lung (Wu et al., 1988; Tashkin et al., 1991). Furthermore, it inhibits alveolar macrophages that contribute to the pulmonary antibacterial defense system (Sherman et al., 1991). On the other hand, heavy use of cannabis is generally defined as smoking at least once per day while, for tobacco smokers, it usually means at least 20 times per day. The total daily inhaled amount of tar is therefore probably much less in heavy smokers of cannabis than of tobacco. Nevertheless, chronic cannabis smoking has the potential to produce bronchitis, emphysema, or pulmonary carcinoma. Furthermore, the cardiovascular and respiratory depressant effects of a single dose are potentially dangerous for patients with pre-existing cardiovascular or pulmonary disease. 
Changes in reproductive functions have been a source of controversy ever since Kolodny et al.'s (1974) widely publicized report. It indicated that chronic male marijuana users had depressed testosterone levels and also that follicle stimulating hormone levels were lower in heavy than light users. Further investigations showed depressed levels of other gonadotropins in chronic THC users, specifically luteinizing hormone and prolactin. But, Block et al. (1991) noted that they were outnumbered by studies showing no significant difference in any hormone's concentration between groups of users and nonusers. They went on to measure testosterone, luteinizing hormone, follicle stimulating hormone, and prolactin concentrations in 27 frequent, 18 moderate, and 30 infrequent marijuana users, and compared them with the values obtained from 74 nonusers. Also measured were cortisol concentrations to control for possible reactions to the stress of venipuncture. No significant intergroup differences for any of the five hormones were found in either men or women.

While cannabis' effects on hormone levels seem to be minor, those on other reproductive functions appear to be of greater concern. Adverse effects on males' sperm have been repeatedly reported. These include decreased sperm count and motility, and increased morphological abnormalities (Kolodny et al., 1974; Hembree et al., 1979). These early findings are corroborated by the recent discovery of cannabinoid receptors, though sparse, in human testis (Gérard et al., 1991). In addition, THC crosses the placenta (Idanpaan-Heikkila et al., 1969) and may retard fetal growth (Zuckerman et al., 1989; Day et al., 1992). Newborns prenatally exposed to cannabis had, compared to controls, decreased mean arm circumference and nonfat volume of the arm, but normal fat stores (Frank et al., 1990). The fetal growth retardation followed a symmetric pattern and no abnormalities were found in weight/length or arm circumference/head circumference ratios, which would have indicated a malnutritional cause. Because THC decreases fetal oxygenation (e.g. Clapp et al., 1987), Frank et al. were led to hypothesize that maternal-fetal hypoxia produced the retardation of fetal growth. One study reported that offspring of women who chronically used marijuana during pregnancy were more likely to have abnormalities comparable to the fetal alcohol syndrome than offspring of nonusers (Hingson et al., 1982), but another showed that infant morphological abnormalities could not be explained by the mothers' marijuana use (Day et al., 1992). Exposure to cannabinoids via the mother's milk during the first month postpartum was associated with decreased motor development at one year of age (Astley and Little, 1990).

Canadian investigators recorded the pregnancies and deliveries of approximately 700 women residing in the Ottawa region over a six year period. They also failed to find differences between users and nonusers with regard to pregnancy outcome measures, including miscarriage rates, type of presentation at birth, Apgar status, and frequency of complications or major physical anomalies of the newborn infants (Fried et al., 1983, 1984). Behavioral evaluations revealed that 
prenatally exposed newborns had increased tremors and startles, and poorer habituation to visual stimuli (Fried and Makin, 1987). At one, two, and three years of age, no adverse effects of prenatal THC exposure were found (Fried, 1989a). One of Fried's (1989b) alternative explanations was that "the drug's effects are transitory and ... the nervous system has 'caught up' sufficiently in the marijuana offspring to be undifferentiated at a behavioral level from control subjects." Yet at four years of age, children of mothers who smoked marijuana during pregnancy yielded significantly lower scores in verbal and memory domains than the controls (Fried and Watkinson, 1990). At six years of age, they committed more omission errors in a vigilance task and had higher ratings by the mothers on an impulsive/hyperactive scale (Fried et al., 1992). It may be, as Fried (1989b) stated earlier: "the long-term effects of maternal marijuana use are very subtle and the facets of behavior that are affected manifest themselves under more complex situations than can be examined in a very young child." Opposed to these results are those of a Jamaican study which failed to reveal any adverse effects of prenatal marijuana exposure on 4- and 5-year old children's abilities (Hayes et al., 1991).

Most studies controlled at least for some potential confounding factors, such as socioeconomic status, other drug use, and home environment. There are, however, other variables that are more difficult to control but may influence newborn and later development, including diet during pregnancy and the postnatal parent-child relationship (Fried, 1989a; Zuckerman and Bresnahan, 1991). These investigators suggested that poor diet during pregnancy and deficient child caring may potentiate the adverse effects of prenatal drug exposure. Conversely, children might be protected from the drug's effects in utero if their mothers' diet during pregnancy is optimal; and, might recover from adverse effects, if any, when an intervention program addressing the child's developmental needs is provided in combination with a resolution of the mother's, and, if pertinent, also the father's, drug dependence.

In conclusion, chronic use of cannabis has been associated with many adverse effects, including the psychological, physical, and teratological. While the acute effects of cannabis were relatively simple to establish, it has proven very difficult to find evidence for the alleged long-term consequences of cannabis use, particularly the psychopathological. This is not surprising. Long-term effects generally appear only after many years of sustained drug use, and, in the meantime, many other factors confound the observed association between cannabis use and adverse effect. Even when a true association is found, it's still open to question whether cannabis use was the cause or simply an effect. Only prospective studies including very large samples seem capable to demonstrate long-term effects of drugs in general, and cannabis in particular. Excellent examples of these type of studies were provided by Shedler and Block (1990) and the Ottawa group. On the basis of the available evidence, it appears that cannabis 
does not produce antisocial or criminal behavior, is not a stepping stone to dependence on more harmful drugs, and produces neither psychosis nor amotivational syndrome in normal, stable individuals. It seems that heavy use of cannabis can be just one among the many symptoms of maladjustment or psychopathology. At most, cannabis may exacerbate an individual's vulnerability and provoke pre-existing or aggravate existing psychiatric illness. On the other hand, chronic cannabis use has been associated with some cognitive and psychomotor deficits. While it remains to be determined whether these effects are solely attributable to chronic drug use, it would be premature to draw definite conclusions regarding the psychological sequelae of long-term cannabis use. Perhaps of greater importance are the physical consequences. Cannabis has the potential to suppress the immune system, produce respiratory disease, and increase the risk of lung cancer. Furthermore, it may impair reproductive functions, retard fetal growth, and possibly the postnatal development of children born (and reared) by cannabis-dependent mothers.

\subsubsection{Tolerance and Dependence}

An important question with relation to cannabis, as to all other psychoactive drugs, is whether long-term use produces tolerance and/or dependence. This has important implications for both heavy recreational users and patients who use THC therapeutically (below). Tolerance to some drug effects may be desired by one group but not the other. The possible experience of withdrawal symptoms after abrupt drug discontinuation is disliked by all users.

Tolerance means that a drug's effects diminish with repeated administration of similar doses; and, that greater amounts must be administered to produce the effects initially experienced. Most psychoactive drugs including THC have multiple effects. Tolerance generally does not develop to all of them at the same rate so it should be determined for each effect separately. Dependence can be defined as (1) preoccupation with drug acquisition, (2) compulsive use, and (3) relapse to or recurrent use (e.g. Miller and Gold, 1989). Physical or physiological dependence on a drug is said to have developed when a characteristic abstinence syndrome is observed when use of that drug is discontinued; and, when the withdrawal symptoms can be reversed by readministration of the drug. Physical dependence has previously been referred to by the term addiction. The concept of psychological dependence, previously referred to by the term drug habituation, was proposed to describe drug dependence without the occurrence of withdrawal reactions when drug use is terminated. Individuals with a psychological dependence on a drug may, if the drug is withdrawn, exhibit reactions that resemble those of a withdrawal syndrome, e.g. restlessness and irritability, but should not be regarded as such unless tolerance has also developed (Davison and Neale, 
1978). Although tolerance and dependence are closely related, the former does not necessarily produce the latter.

Diminished drug-induced tachycardia and psychological 'high' have been observed by several investigators after repeated marijuana smoking within a relatively short period, i.e. 2 to 18 hours (Cochetto et al., 1981; Heishman et al., 1990; Chait, 1990). These studies were, however, not specifically designed to examine the possible development of tolerance. Those that were suggested tolerance without conclusive evidence because ethical considerations limited the subjects' consumption of THC in repeated doses (Hollister, 1986). Jones and his colleagues conducted a series of outstanding studies to examine tolerance and withdrawal symptoms following prolonged oral administration of THC. Jones et al. (1981) summarized their results by concluding, "both tolerance and physical dependence develop after surprisingly short periods of cannabis or THC administration when the conditions are optimal", i.e. when dose, dosing frequency, duration, and route of administration are such that sustained blood/brain levels of THC are achieved. Their volunteers, all experienced marijuana users, lived on a hospital research ward for two to six weeks. They usually received placebo treatment for three to seven days after admission, followed by a 5- to 21-day period of active treatment with 10 to $30 \mathrm{mg}$ oral doses of cannabis extract or THC, given every three or four hours, 24 hours a day. Then, under double-blind conditions, treatment was abruptly switched to placebo for four to eight days. Tolerance to THC's subjective effects developed rapidly. For example, a $50 \%$ reduction in intoxication ratings was observed after four days exposure to $10 \mathrm{mg}$ doses. More than $80 \%$ reduction was achieved in the late stage of drug administration. Initial physiological signs of intoxication such as tachycardia and decreased skin temperature also gradually disappeared, with bradycardia developing at the end of the treatment period. Tolerance to orthostatic hypotension developed within 12 to 24 hours. Initial decreases in intraocular pressure, salivary flow, and REM sleep partially or completely recovered within ten days of sustained drug administration, and rebounded above pre-drug levels after abrupt cessation. Tolerance did not develop to the drug's appetite-enhancing effect and body weight increased progressively. Tolerance was further examined while subjects received constant oral doses of $20 \mathrm{mg}$ every three hours or $30 \mathrm{mg}$ every four hours and were challenged daily with superimposed doses of $20 \mathrm{mg}$ via marijuana smoking or $2 \mathrm{mg} / 70 \mathrm{~kg}$ via injection. Smoked or injected THC doses were also administered before the oral treatment period to measure their acute effects, and, after withdrawal of oral treatment, to determine how long tolerance persisted. Subjective, heart rate, skin temperature, and intraocular pressure reactions to the challenge diminished after only a few days of oral THC administration, but initial reactions rapidly returned when placebo was substituted for drug in oral dosing. Another interesting finding was that tolerance developed more rapidly while the subjects were constantly treated with the lower, more 
frequent oral doses than the opposite. According to Jones et al., this suggests that the rate of tolerance acquisition is mainly a function of the time subjects are in the rapid $\alpha$-phase of THC elimination from plasma rather than the $\beta$-phase with the slower elimination.

Tolerance to a drug's effect generally implies that progressively larger arnounts of the drug must be taken to reproduce its initial effects. Yet Cappell and Pliner (1974) found no difference between frequent and infrequent users' self-administration of marijuana cigarettes to achieve a 'nice high'. This indicates that the regularity of marijuana smoking by those who described their use as 'frequent' is still not sufficient for acquiring substantial tolerance. If marked tolerance to THC's subjective as well as cognitive and psychomotor effects were common, there would be implications for experimental research: subjects whose smoking regularity differed widely could not be considered as members of the same population and conclusions would necessarily be confined to that subpopulation providing the results. However, the general lack of tolerance exhibited by recreational THC users and the usual periods of abstinence required by most experimental protocols prior to testing encourage the belief that acute effects are not confounded by that factor.

The nature of tolerance may be either pharmacodynamic or pharmacokinetic. The former, also called 'functional' tolerance, is due to localized changes at the drug's site of action. The latter, also called 'dispositional' tolerance, is a result of either increased drug metabolism or other factors that reduce its plasma concentration after repeated dosing. Hunt and Jones (1980) administered ${ }^{14} \mathrm{C}$-labelled THC by i.v. injection in six subjects both before and at the end of the oral THC administration period, and found only a slight increase in drug metabolism, too little to account for the observed degree of tolerance. Furthermore, there seems to be no difference in the rate of clearance of THC between chronic and naive users (Agurell et al., 1986). The available evidence therefore indicates that tolerance to THC is functional rather than dispositional in nature.

In Jones' studies, withdrawal signs and symptoms began to appear five to six hours after the last oral THC administration. Physiological signs such as salivary flow, intraocular pressure, REM sleep, finger tremor, and sweating were increased, body weight decreased. Subjective symptoms included irritability, restlessness, insomnia, anorexia, and nausea. Both physiological and subjective withdrawal phenomena were alleviated for two to three hours by smoking a marijuana cigarette containing $20 \mathrm{mg}$ THC. Generally, symptoms were most intense at about eight to twelve hours after THC cessation, diminished thereafter, and were not measurable three to four days later. The exception was disturbed sleep: some subjects reported insomnia for as long as several weeks. Other studies, reviewed by Hollister (1986) and Compton et al. (1990), wherein THC was inhaled or where smaller doses of THC were administered orally, found fewer and milder symptoms of a similar nature during withdrawal. There are apparently no reports 
of deaths during withdrawal but many describe psychological dependence on the drug. Therefore, Compton et al. concluded that psychological dependence is more probable and of greater importance than physical dependence. This opinion was endorsed by Shedler and Block (1990). Their findings suggested that dependence upon cannabis is a symptom rather than a cause of social and personal maladjustment. Grinspoon and Bakalar (1992) articulated this view as follows: "becoming attached to cannabis is not so much a function of any inherent psychopharmacologic property of the drug as it is emotionally driven by the underlying psychopathology (p. 243)."

Both tolerance and withdrawal depend upon the THC dose taken at one time, and the frequency and duration of dosing. These phenomena probably occur in a coupled series; substantial tolerance followed by strong withdrawal symptoms. But neither is likely to become practically significant until large THC doses are consumed more than once daily over a prolonged period of time. According to Hollister, most users do not follow such a dosing regimen. So neither tolerance nor physical dependence has been a major issue in social cannabis use. This may change with the recent rise in cannabis potency. As cannabis smokers seem not to adjust their smoking pattern to the drug's potency (see Section 2.5.2), instances of physical dependence may become more prevalent in the near future.

\subsubsection{Therapeutic Applications}

The most promising therapeutic use of THC is for treatment of nausea and vomiting associated with cancer chemotherapy (Chang et al., 1979; Vinciguerra et al., 1988; Randall, 1990). Two synthetic cannabinoids, nabilone and levonantradol, are also effective antiemetics (Vincent et al., 1983). Dronabinol, formulated in sesame oil and encapsulated in round soft gelatin capsules for oral administration, was approved in 1985 for antiemetic indications by the FDA. The drug is now marketed under the name Marinol ${ }^{*}$ (Unimed Inc., Somerville, NJ).

THC has also been successfully applied in some cases of anorexia nervosa (Zinberg, 1979), and clinical trials have demonstrated that patients suffering from AIDS may also benefit from the drug's appetite stimulating effects (Plasse et al., 1991). In 1993, the FDA approved of a supplemental drug application for dronabinol for use in AIDS patients who suffer anorexia and weight loss.

THC decreases intraocular pressure by decreasing fluid formation and increasing fluid outflow. It has been successfully applied in the treatment of glaucoma or ocular hypertension (Merrit et al., 1980; Grinspoon and Bakalar, 1993).

A variety of THC indications have been suggested besides those already accepted by drug regulatory authorities. Although cannabinoids do not bind at opiate receptors, levonantradol and its active metabolite desacetyllevonantradol 
were found to be potent analgetics in rodents (Howlett et al., 1990). THC and CBD posses anticonvulsant properties which may be of therapeutic value (Relman, 1982; Hollister, 1986). They exacerbate hypokinesia and resting tremor in Parkinsonian patients but are beneficial for some forms of dystonia, tremor, and spasticity (Petro and Ellenberger, 1981; Consroe et al., 1986; Meinck et al., 1989). CBD attenuates the anxiogenic effect of THC and possesses anxiolytic properties in individuals exposed to a stressful situation (Zuardi et al., 1982, 1993). Other alleged indications for cannabinoids include bronchitis, asthma, insomnia, hypertension, abstinence syndromes, migraine, and alcoholism. But approval of cannabinoids for any of these indications is, at present, most unlikely (Hollister, 1986).

\subsection{Summary and Conclusions}

Man's interest in Cannabis sativa extends backward for more than ten thousand years. Cannabis has been used as an ingredient in clothes and rope, and as a medicine for a variety of discomforts and diseases. The principal interest in cannabis, however, has been in its euphoric and mind-altering properties. In Western societies, recreational use of cannabis for that reason began in the Middle Ages, but it was not until the 1960s that cannabis' popularity became widespread, not only in lower social classes and minority groups but throughout all levels in society. As a consequence, scientific research on cannabis increased dramatically ever since.

Marijuana and hashish are the most widely used cannabis preparations in Western societies. The former is made from the dried leaves and flowering tops of the plant, which are cut, dried, chopped, and incorporated into cigarettes. The latter is prepared from the resinous exudate itself and is often mixed with tobacco before smoking. The drug can also be chewed, prepared as tea, or eaten in baked goods.

Cannabis sativa contains more than 400 chemical compounds. More 60 , the cannabinoids, are specific to the plant and are present in the sticky golden resin that covers the flowers and top leaves. The cannabinoid primarily responsible for the drug's physiological and psychological effects is $\Delta^{3}$-tetrahydrocannabinol (THC). When entering the systemic circulation, THC is rapidly distributed into highly perfused fatty tissues, including the brain. Next, it slowly diffuses from tissue into blood, is metabolized and excreted in urine and feces. The distribution $(\alpha)$ phase has a half-life of 30 minutes, the elimination $(\beta)$ phase, several days. As a result, accumulation of THC occurs with repeated drug administration, though only in neutral fat and liver and not in blood and brain. Due to their slow elimination, THC's metabolites can be present in urine for weeks after the last drug intake. Recently, cannabinoid receptors have been identified in human 
brain, testis, spleen, tonsils and leukocytes. Receptor concentrations are most dense in certain brain areas, including the cerebral cortex, hippocampus, cerebellum, and projection nuclei of the basal ganglia. Furthermore, a putative endogenous ligand has been discovered. These findings, and others to come, will certainly contribute to a better understanding of the cannabinoids' mechanism of action.

The acute subjective effects of cannabis smoking appear within 15 minutes and generally last for about two to four hours. When cannabis is ingested, THC's onset of action is some hours delayed and its effects generally last for 5 to 12 hours. The most reliable physiological signs of cannabis intoxication include conjunctivitis and tachycardia. The principal psychological effect is an euphoric state which is consistently accompanied by distorted time perception and disruption of memory processes. The user may experience temporal disintegration, manifested as difficulty concentrating and intrusion of thoughts, and depersonalization. Dysphoric reactions may occur in inexperienced users or anyone after consuming very high doses of THC and include anxiety, sometimes accompanied by paranoid thoughts, and panic reactions. Acute depressive episodes and toxic psychosis have been occasionally reported, mainly in users with some underlying depression or a history of psychosis.

The timing and magnitude of the acute effects are dependent on the THC dose and route of administration. Other factors that may influence the acute effects of THC, particularly when consuming low or moderate doses, include the social setting and the user's expectations based on previous experience. The smoking technique may affect physiological signs of intoxication, but has little effect on subjective feelings or performance.

It has proven very difficult to find convincing evidence for the alleged longterm sequelae of cannabis use. Heavy chronic users of cannabis have been shown to be socially and personally maladjusted, but a longitudinal study indicated that problem drug use is more likely a symptom rather than the cause of maladjustment. While cannabis use may provoke pre-existing or aggravate existing psychiatric illness, there is no convincing evidence that it produces functional psychosis de novo in a stable individual. Neither is there much reason to believe that cannabis use per se leads to criminal behavior, dependence on other, more harmful drugs, or amotivational syndrome. Yet chronic cannabis use was associated with some cognitive and psychomotor deficits. It remains to be determined whether these effects are solely attributable to cannabis but they are probably the most obvious psychological sequelae of chronic cannabis use. The physical sequelae seem to be more pervasive. Chronic use of cannabis suppresses the immune system and may produce respiratory disease and lung cancer. Furthermore, it reduces sperm quality and may retard both fetal growth and postnatal development. 
Both tolerance and dependence followed by withdrawal symptoms are a function of the dosing regimen. Neither is likely to reach clinically significant levels until large THC doses are consumed more than once daily over a prolonged period of time. Tolerance appears more functional than dispositional and is rapidly reversible with abstinence. Withdrawal symptomatology is mild and transient, and is characterized by irritability, restlessness, insomnia, anorexia, nausea, salivation, sweating, tremor, increased intraocular pressure and increased REM sleep.

The most promising therapeutic applications of THC include the treatment of nausea and vomiting in cancer patients undergoing chemotherapy, and anorexia associated with weight loss in AIDS patients. Cannabinoids have also been shown to possess analgesic and anticonvulsant properties, and may be successfully applied in the treatment of glaucoma and some forms of dystonia, tremor, and spasticity.

In conclusion, cannabis is a notorious drug but also a fascinating pharmacological entity. Throughout history many people have shown interest in the drug for many different reasons. Lately that interest has been shared by scientists resulting in a huge number of publications. Yet there is still a surprising paucity of sound information regarding the long-term effects of cannabis. Consequently, many people's viewpoints are still largely based upon personal experience or prejudice, or both, which has resulted in extreme polarization.

Similar opposing opinions prevail regarding cannabis' effects on driving performance and traffic safety. Some apparently believe that cannabis users are among the most hazardous drivers on the road whereas others claim that cannabis produces more cautious driving and is therefore no hazard at all. The debate might continue indefinitely were one unable to objectively measure THC's effects on its users' actual driving performance. Fortunately, it was possible for us to do so in a series of acute dosing studies conducted in environments that gradually approached full ecological validity. Before describing them, however, it seems wise to consider what previous research has shown concerning the influence of cannabis smoking on driving. This is done in the following chapter with a literature review focused upon that specific issue. 


\section{CHAPTER 3}

\section{CANNABIS AND DRIVING}

THC's effects on the ability of drivers to operate safely in traffic situations have traditionally been determined in two ways: from epidemiological surveys of users' involvement in traffic accidents and from empirical studies to measure the drug's influence on skills related to driving. The results obtained from both approaches are briefly reviewed for the purpose of providing a justification for our own research described in the following chapters.

\subsection{Epidemiological Studies}

The purpose of epidemiological studies on cannabis and traffic safety is to determine if driving under the influence of cannabis is detrimental to the safe operation of motor vehicles. Essentially, they determine if cannabis use is overrepresented in drivers who were involved in traffic accidents.

Simpson (1986) has reviewed recent epidemiological evidence regarding cannabis' role in traffic accident causality. His first concern was to determine the frequency of driving shortly after cannabis use to identify the proportion of the total driving population who may be considered 'at risk' of causing an accident for that reason. His information was derived from two sources: questionnaire surveys of adolescents (16-19 years), who were licensed to drive, and roadside surveys of recent usage among passing motorists.

He mentioned the reasonably consistent results of three questionnaire surveys conducted in the United States or Canada between 1979 and 1982. About one in six teenage drivers admitted driving while smoking or shortly afterward, and about $10 \%$ said they had done so between one and five times during the preceding month. Similar results were reported by Burns (1981): $25 \%$ of $16-21$ years old American marijuana users admitted having driven after drug use. But in Valladolid, Spain, Alvarez et al. (1991) found that only $3.4 \%$ of drivers between 18 and 70 years of age admitted having driven a vehicle at least once after taking an illegal drug. Cannabis alone or in combination with another drug was used in $61.5 \%$ of these cases. The percentage of young (18-24 years) Spanish who 
admitted driving after illegal drug consumption was closer to those in North America. Overall this rate was $4.5 \%$ and it increased with the young Spanish drivers' educational and, presumably, socioeconomic status. The separate incidence for cannabis was not reported. These studies show that at least some, primarily young, users do drive during or shortly after cannabis smoking. Unfortunately it is not possible to generalize the results of the former studies to older drivers in North America, nor those of the latter study to other countries in Europe.

Only three surveys of recent cannabis use among drivers stopped at roadside check points have apparently been reported. According to Simpson, one yielded uninterpretable results due to technical and methodological problems. The others were widely separated in place and time. The first was completed in Canada in 1974 (Smith et al., 1975), the second in Italy in 1982 (Ferrara and Rozza, 1985). Moreover, the former relied upon the drivers' admission of use and the latter upon detection of cannabinoids in urine samples. Nonetheless, the indications of recent cannabis use given by the two sets of results were not grossly different; $4 \%$ by the first and $1.2 \%$ by the second.

Terhune (1982) tested 497 injured drivers for the presence of a wide range of drugs during treatment at the Rochester General Hospital in New York. THC in blood was detected in $9.5 \%$ of the drivers, but more than half of them also tested positively for alcohol. Chesher and Starmer (1983) found THC in $6.7 \%$ of 104 injured drivers in New South Wales, Australia, but again about half of them showed alcohol as well. Daldrup et al. (1987) examined 597 blood samples from injured drivers in the region around the German city Düsseldorf for the presence of alcohol. Blood samples having alcohol concentrations (BAC) below $0.13 \mathrm{~g} \%$ were additionally analyzed for the presence of cannabinoids; twenty-five of the 220 blood samples (11.4\%) were positive. Finally, McLean et al. (1987) found THC in four out of $37(10.8 \%)$ drivers who had survived a road accident in Tasmania. Three of them also had a BAC of $.05 \mathrm{~g} \%$, or more. Although the latter sample was very small, the percentage of injured drivers with THC in their blood corresponds remarkably well with the other studies. Together they agreed that THC is present in the blood of about $6-12 \%$ of drivers injured in road accidents.

These data contrast substantially with those provided by Soderstrom et al. (1988) from 1023 patients admitted as the result of vehicular and nonvehicular accidents at the Shock Trauma Center in Baltimore, Maryland. THC was found in blood in $34.7 \%$ of the patients, alcohol in $33.5 \%$. Among automobile drivers, the numbers were $31.7 \%$ and $34.6 \%$, respectively. Both drugs were present in about $50 \%$ of the injured drivers. It is not clear why these results differ from those of previous studies. The most plausible explanation is that residents of the Baltimore area tend, in general, to use THC more often than those in the other regions surveyed. Investigators at two other trauma centers (Philadelphia and Chicago) found similar incidence rates. However, they measured all cannabinoids 
instead of only THC in either blood (Lindenbaum et al., 1989) or urine (Sloan et al., 1989). The incidence of THC in trauma patients at these locations must have been much lower than in Baltimore.

Cimbura et al. $(1980,1982)$ analyzed the blood of 401 fatally injured drivers in the province of Ontario, Canada, during 1978-1979. THC was detected in $3.7 \%$ but alcohol was also found in most of the THC positive cases, i.e. $87 \%$. In their subsequent study in the same area during 1982-1984, the investigators reported that THC was detected in blood among $10.9 \%$ of 1169 fatally injured drivers (Donelson et al., 1985; Cimbura et al., 1990). Again, more than $80 \%$ of THC positive blood samples also contained alcohol. Mason and McBay (1984) found THC in $7.8 \%$ of 600 drivers killed during single-vehicle crashes occurring in North Carolina during 1978-1981. Preliminary results from this study have been published by Owens (1981) who reported a $5.9 \%$ incidence rate in 169 driver fatalities during 1978-1979. As in Ontario, the vast majority of THC positive drivers had also used alcohol. Two studies employing very small samples have also been reported. In the first, 11 out of 69 drivers (15.9\%) who were killed in traffic accidents in Bexar County, Texas, during 1985 had THC in their blood (Garriott et al., 1986). In the second, four out of 42 road-accident fatalities (9.5\%) in Tasmania during 1983-1984 were positive for THC (McLean et al., 1987).

Disparate results were obtained by Williams et al. (1985): THC was found in $36.8 \%$ of 440 driving fatalities in California during 1982 and 1983 (80\% in combination with alcohol). The reason for this disparity might have been due to the greater prevalence of marijuana use in California as compared to other American States. But it was more probably related to these investigators' selection criteria: their sample only included male drivers younger than 35 years of age. Simpson estimated that if female and older male fatalities had been included in this survey, the overall percentage showing THC would have been about $20 \%$. Moreover, $38 \%$ of the THC positive drivers showed only trace amounts of the drug, i.e. less than $1.0 \mathrm{ng} / \mathrm{ml}$. If these cases are viewed as false positives, according to the conventional epidemiological criterion, the number of THC positive drivers would be $22.8 \%$ in the original sample, and only $12.4 \%$ after Simpson's correction. More recent Californian data were provided by Budd et al. (1989). They assayed various drugs in the blood of drivers killed in Los Angeles County, both in a preliminary study during 1985-1986 and in a follow-up study over the next two years. Both times they found cannabinoids (THC and/or THC$\mathrm{COOH}$ ) in about $19 \%$ of dead drivers (total $\mathrm{N}=313$ ). One can only guess what the incidence rate for THC alone might have been on the basis of cases showing both the parent compound and its metabolite. Probably it was somewhere between 10 and $13 \%$, which corresponds well with the previous studies.

The most recent data regarding the incidence of drugs in fatally injured drivers in the United States are available from a nationwide study conducted in 1990 and 1991. Blood specimens were collected from 1,882 dead drivers from 13 sampling 
sites, encompassing three entire states (Massachusetts, North Carolina, and Wisconsin) and selected counties in California, Nevada, Texas, and Virginia (Terhune et al., 1992). THC was found in only $4.2 \%$ of the drivers, and about $70 \%$ of the time in combination with alcohol. THC alone was found in only 19 fatalities or $1 \%$ of the total sample. These relatively low percentages may indicate a declining trend in the incidence rate of THC in fatally injured drivers in the United States, explainable by the declining prevalence of marijuana use throughout the 1980s.

With some exceptions, epidemiological studies indicate the presence of THC in roughly $4-12 \%$ of drivers injured or killed in traffic accidents. If one accepts that the population at risk is less than $4 \%$, the injury/fatality rate must be taken to indicate that the drug's users are overrepresented among accident victims. However, there are obvious reasons to doubt whether valid estimates of the population at risk in urban North America can be derived from data that are more than 14 years old or were obtained at two locations in Northern Italy. Even if the population at risk is as small as estimated, the THC incidence among injured or dead drivers is not conclusive evidence for establishing its role as a causal factor. Alcohol was also present in the majority of survey victims showing any plasma concentrations of THC. It is highly likely that these drugs in combination possess a greater risk potential than either alone. The independent contribution of THC to traffic accident causality, particularly in concentrations which are likely to be found in most users, is still dubious.

One major problem of these surveys is the common lack of sound control groups as have been used in studies of alcohol involvement in accidents (e.g. Borkenstein et al., 1974). BACs measured in fatal accident victims were compared to breathalyzer BAC estimates from randomly selected motorists passing the same accident sites at the same times and days of the week. That comparison provided a ratio of fatally injured drivers to normal controls showing a given BAC and how it varies across the range found in all drivers. This approach was feasible because nearly all $(>97 \%)$ passing motorists were willing to yield breath samples; and valid, because alcohol concentration in the blood is about the same as in the brain. Many fewer motorists are prepared to yield blood samples for a variety of reasons, including that of revealing illicit drug use. Moreover, THC concentrations in blood and brain are almost always different. As Moskowitz (1985) observed, these fundamental feasibility and validity premises which were met in alcohol surveys can not be in surveys for measuring the relationship between THC and accidents.

The lack of separate control groups can be circumvented by the use of a culpability index. The index is the ratio of the percentage of drivers with detectable drug levels and deemed culpable to that of drug-free drivers from the same sample who were likewise responsible for their accident. Warren et al. (1981) reanalyzed the data from Cimbura et al. (1980) showing that $52 \%$ of the 
drug-free fatally injured drivers were deemed culpable compared to $90 \%$ of those with evidence of recent cannabis use. This yielded a culpability index of 1.7 , the same as for alcohol. Results of two other studies (Terhune, 1982; Donelson et al., 1985) were consistent with these findings. On the other hand, Williams et al. (1985) and Terhune et al. (1992) failed to find a significantly elevated culpability index for THC users. In the latter study $58 \%$ of the drivers in whom only THC was detected were deemed culpable, whereas $68 \%$ of the drug-free drivers were. The difference in favor of THC users was not significant. In contrast, dead drivers showing only alcohol were culpable in $92 \%$ of all cases. Those showing both drugs were slightly more often responsible for causing the accident (95\%). It should be noted, however, that the usually low frequencies of culpable drivers showing THC alone prohibit any definite conclusion.

In summary, epidemiological research has shown that some people do drive after cannabis use and that drivers involved in accidents often show the drug's presence. However, alcohol has been a severe confounding factor in all surveys of injured or killed drivers. For this and other reasons given above, the independent contribution of THC to their accidents remains exceedingly obscure.

\subsection{Laboratory Studies of Skills Related to Driving}

Much research has gone on to determine the drug's effects on isolated psychological functions and skills related to driving since cannabis' revival in the 1960 s. Owing largely to the fact that its methodology already existed and could be economically applied, this research preceded epidemiological surveys and actual driving studies. Yet one of the major drawbacks of laboratory research is that no performance model comprehensively defines the actual driving task. As a consequence, different researchers have employed different part-task models to design a plethora of tests. This was clearly illustrated by Joscelyn et al. (1980) who inventoried the methods applied in the drugs and driving research and came up with long lists of tests, tasks, and response variables. They noted: "many tests routinely employed have limited validity or no demonstrable relation to realworld driving. Methods measuring the 'same' behaviors often differ, raising questions about the comparability of experimental findings (p. 34)."

This does not mean that laboratory studies are useless for assessing the drugs and driving problem. On the contrary, they provide the earliest evidence concerning a particular drug's hazard potential for driving. In the context of well designed experiments, drugs that produce large performance impairments in many different tests can be considered potentially hazardous whereas drugs that fail to produce any impairment can be considered safe. Yet results obtained in the laboratory should not form the sole basis for the final judgment of a drug's potential to impair driving performance and jeopardize traffic safety. They 
should be confirmed, if possible, by epidemiological surveys and actual driving studies.

Two recent reviews of the literature pertaining to the effects of marijuana on skills related to driving contain most of the information pertinent to this dissertation (Moskowitz, 1985; Chesher, 1986). No attempt will be made to replicate their comprehensive efforts. Instead their major conclusions will be mentioned along with certain contradictions and omissions in the available data base. Where appropriate, the results of more recent studies are also mentioned.

\section{Visual functions}

Visual acquisition of information is the first step in the process of controlling a motor vehicle. For that reason it seems appropriate to first answer the question of whether either visual functions or oculomotor coordination is impaired by THC. In general, studies reviewed by Moskowitz have shown little or no effect for 0-6 hours of ingested THC doses up to about $300 \mu \mathrm{g} / \mathrm{kg}$, or inhaled doses up to about $15 \mathrm{mg}$ ( $210 \mu \mathrm{g} / \mathrm{kg}$ in a $70 \mathrm{~kg}$ person) on static or dynamic visual acuity, binocular fusion, lateral phoria, glare recovery, color vision or saccadic and ballistic eye movements involved in visual tracking. Stapleton et al. (1986) reviewed the effects of psychotropic drugs on eye movements and came to a similar conclusion: unlike alcohol, marijuana has relatively little effect on oculomotor functions.

A curious finding was reported by Schwin et al. (1974) who measured critical flicker fusion frequency (CFF) in subjects who inhaled $15 \mathrm{mg} \mathrm{THC}$. This treatment produced a rise in CFF, the opposite of what one would expect for any drug with depressant CNS properties. Yet Block et al. (1992) found a CFF decrease after subjects smoked a marijuana cigarette containing $19 \mathrm{mg}$ THC.

\section{Fine and Gross Motor Control}

Several studies have shown that even very low inhaled THC doses (e.g. $10 \mu \mathrm{g} / \mathrm{kg}$ ) increase hand or whole body instability in tests such as maintaining the position of a stylus in a hole without touching the sides, or balancing on a platform supported by a central fulcrum. These impairments are dose-related so it seems clear that one of the drug's effects is to impede, or otherwise interfere with the neuromuscular-proprioceptive servocontrol loop. Unless the marijuana user is also a watchmaker or tightrope walker, it seems unlikely that he would suffer greatly from this effect in any practical task, including driving. However, the sensitivity of motor coordination measures and their systematic change with THC dose, led Moskowitz to suggest (p. 328) that one or more might prove useful for identifying states of marijuana intoxication that are incompatible with safe driving. 


\section{Reaction Time}

Simple reaction time is only slightly affected by even relatively high THC doses. In various complex reaction time test paradigms, where the association between the given stimulus and correct response varies between trials and requires an intervening decision on the subject's part, THC's effects have been sometimes large, sometimes small but significant, and occasionally absent. Generally however, the variability of complex reaction times increased under the influence of inhaled THC doses in the range, $100-250 \mu \mathrm{g} / \mathrm{kg}$.

Moskowitz was somewhat at a loss to explain the contradictory reports concerning THC's effects on complex reaction time. These seemed to depend upon the particular test paradigms employed by various groups of investigators. Their diversity without any clear indication of what information processing stages each one measures, leaves one in doubt concerning the meaning of these results. Moskowitz could conclude that moderate THC doses impair neither the initial perception of an unambiguous and anticipated signal, nor the final motor responding speed. However, the elevated variability in complex reaction times suggested to him impairment in the subjects' mechanism of attention to the task.

Some idea of the complexity of measuring THC's influence on complex reaction time, as well as the suggestion of a fundamental drug effect, can be gathered from a study of Chesher et al. (1986). These investigators employed a mental rotation task, i.e. the 'little men' test. A figure of a man holding an object in one extended hand appeared on a computer terminal. On successive trials the figure could be upright or inverted, facing forward or backward and its hand holding the object could be the right or the left. The subject's task upon seeing each new figure was to make one of two button pressing responses to indicate which hand held the object. The frequencies of correct and incorrect responses were measured along with the associated reaction times. There were $16 \mathrm{ex}$ perimental conditions defined by separate combinations of ingested alcohol $(0.00$, $0.25,0.50$ and $0.75 \mathrm{~g} / \mathrm{kg})$ and inhaled THC $(0.00,2.5,5.0$ and $10.0 \mathrm{mg})$. A different group of 20 subjects, who had previously used both drugs, participated in each condition. Chesher's (1986, p. 114) description of the difference between the drug's effects is illuminating.

"The results for the mental rotation test indicated an interesting qualitative difference between the two drugs. The nature of the alcohol or marijuana effect differed according to whether the subject's response to the mental rotation task was correct or incorrect. The drug effect for those responses which were correct was similar to those for the other reaction time measures - an increase in reaction time for alcohol but not for marijuana. Indeed, the lowest dose of marijuana indicated a trend towards antagonism of the effect of alcohol. However, the reaction times, and their standard deviations, for the items for which the subjects' responses were incorrect, indicated a qualitatively different drug effect. The mean reaction time for these responses showed a highly significant linear increase across the marijuana doses but not 
across the alcohol doses. Associated with this, alcohol produced a significant, linear effect on increasing the number of errors; marijuana did not. Furthermore, in this measure also, there was a trend for an antagonism of the alcohol effect by the lowest dose of marijuana. One possible interpretation of these different drug effects is that a 'speed-accuracy trade-off' occurred in the alcohol condition and a more cautious approach was applied in the marijuana condition. One might assume that the linear increase in reaction time for these responses under the influence of marijuana could be due to a greater time in pondering the problem. Possibly when faced with a similar problem under the influence of alcohol, the subject is more likely to take a risk and make a hasty guess at the answer. This behaviour resulted in a significant linear increase in the error rate."

This demonstrates what may be a fundamental and specific THC effect: increased caution that results in slower responses to an ambiguous situation.

\section{Tracking}

Moskowitz's review leaves no doubt that THC impairs every type of laboratory tracking performance. This was shown, without exception, in eight separate studies. Degraded tracking performance occurred shortly after inhaled THC doses as low as $3 \mathrm{mg}$ ( $43 \mu \mathrm{g} / \mathrm{kg}$, for $70 \mathrm{~kg}$ persons). After a $200 \mu \mathrm{g} / \mathrm{kg}$ dose, tracking performance impairment persisted continuously for four hours and intermittently for the next four hours. Barnett et al. (1985) found marijuana-induced impairment in a critical instability tracking test for seven hours after smoking, but neither the magnitude nor duration of impairment was related to the administered THC dose (i.e. 100,200 , or $250 \mu \mathrm{g} / \mathrm{kg}$ ).

Surprisingly, in view of the strength and consistency of the effects of THC on laboratory tracking performance, the same effects have been difficult to replicate in tracking tests more resembling car driving (below).

\section{Attention}

Marijuana smoking has two demonstrably adverse effects on attention. One is seen in dual-task situations and the other in vigilance tests. Moskowitz himself was the first to clearly demonstrate these effects. His earliest study (Moskowitz et al., 1972) was designed to measure the effects of 50,100 and $200 \mu \mathrm{g} \mathrm{THC} / \mathrm{kg}$ on subjects' detection responses to peripheral signals while they were counting light flashes as these appeared on a central display. All three doses caused the subjects to detect fewer peripheral signals in a dose-related manner, and the degree of impairment increased with the cadence of counting. With various embellishments, the adverse effects of similar THC doses upon divided attention have been seen again in several studies published since 1985. Barnett et al. (1985) found dose-related impairment in both a central compensatory tracking task and a peripheral visual search-and-recognition task following THC doses of 100,200 , and $250 \mu \mathrm{g} / \mathrm{kg}$. Performance in a divided attention task involving pursuit 
tracking in the upper half of the video monitor, and, searching for a target number, in the lower half, was not affected by smoking a marijuana cigarette containing either 1.3 or $2.7 \%$ THC (Heishman et al., 1989). Yet smoking 25 puffs of a marijuana cigarette containing $3.55 \%$ THC produced a significant prolongation of response latencies to target numbers in the same test (Azorlosa et al., 1992). In another study, subjects had to react to critical changes of two-digit numbers displayed in the central field of vision and one-digit numbers displayed in the periphery by pressing one of four response buttons or a foot pedal. Smoking a marijuana cigarette containing $2.4 \%$ THC reduced the subjects' overall accuracy and prolonged their reaction times (Perez-Reyes et al., 1988). Marks and MacAvoy (1989) employed a divided attention task in which subjects had to react to a break in the regularity of a flashing central light as well as to each flash of one of ten peripheral lights that were spaced at $15^{\circ}$ intervals along a horizontal perimeter. Decrements in both central and peripheral signal detections were found after inhaling 5.2 but not $2.6 \mathrm{mg}$ THC.

Sharma and Moskowitz $(1973,1974)$ further demonstrated the effects of THC on sustained attention as it is traditionally measured in prolonged signal detection or vigilance tasks. Their paradigm was adopted from the famous 'Mackworth Clock Test'. Subjects viewed a number of lights arranged in a $30.5 \mathrm{~cm}$ (12 in) diameter circle. These were illuminated singly in a regular sequence, except occasionally when the progression skipped a particular light. The discontinuity in the sequence was defined as a signal to which the subject responded. Normally subjects show a 'vigilance decrement' or decline in the percentages of detections as a function of time on watch, as did subjects in both experiments after smoking a placebo cigarette. In the first experiment, the decrement accelerated after subjects had inhaled 50,100 and $200 \mu \mathrm{g}$ THC $/ \mathrm{kg}$. Changes from placebo were significant after the two higher doses. One condition in the second experiment replicated the results for the highest dose condition in the first. Another demonstrated that the same decrement occurred when the subjects were required to respond, following every flash, to indicate their perceptions of signals and nonsignals. The stated purpose of the new condition was to show that the subjects were in fact losing their ability to discriminate between stimuli and not simply ceasing to respond because of wandering attention.

\section{Conclusion}

Doses of THC that have been administered in laboratory studies are less than those commonly found in marijuana cigarettes and are apparently sought by drug users to achieve the desired 'high'. Indeed both Moskowitz and Chesher admitted that their highest laboratory doses may be considerably less than those contained in cigarettes manufactured for consumption by normal users. Thus, it should be clear from their brief review that doses normally taken by users possess the potential for impairing at least their neuromuscular coordination, tracking skills 
and attentional functions. Yet there is at least some suggestion from laboratory studies that the subjects attempted to compensate for these deficiencies, in so far as they could, by employing behavioral strategies that maintained the integrity of some performance parameters at a cost to others.

\subsection{Driving Simulator Studies}

Early studies by Crancer et al. (1969), Rafaelsen et al. (1973a), Ellingstad et al. (1973) and Moskowitz et al. (1976) utilized the filmed ride approach where subjects had little or no control over the presented imagery. Dott (1972) used a different approach for measuring subjects' decisions to pass a preceding car, or not, in the presence of an opposing vehicle portrayed as models on a continuous belt. Doses of inhaled THC varied from about 3 to $22.5 \mathrm{mg}$ (43-321 $\mu \mathrm{g} / \mathrm{kg}$ for $70 \mathrm{~kg}$, or $154 \mathrm{lb}$, persons). Smiley (1986) reviewed these early studies to conclude that THC had (1) not affected vehicle control, (2) increased decision latency before starting, stopping or overtaking, (3) reduced the willingness to accept a risk during passing maneuvers, and (4) impaired speedometer monitoring. Except in the case of one individual who, after inhaling $12 \mathrm{mg}$ THC, repeatedly drove through stop lights during a filmed ride, no particular sign of dangerous driving behavior was observed.

Smiley et al. (1981) conducted the first study using an interactive simulator with accurate visual imagery, though not moving base dynamics. The simulated tasks contained in a 45-minute scenario included curve following, reacting to wind gusts, car following, route selection from signs, avoiding an obstacle which appeared in front of the simulated vehicle and passing. A visual choice reaction time was also superimposed on driving. Three groups of marijuana users smoked cigarettes containing 0,100 and $200 \mu \mathrm{g} / \mathrm{kg}$ THC on two occasions per dose, once with and once without alcohol. The quantity of alcohol consumed varied between groups to reach intended blood concentrations of $0.00,0.05$ and $0.08 \mathrm{~g} \%$, respectively. To ensure high motivation, good driving was rewarded and blatant errors, such as crashes, were penalized financially. The test began 15 minutes after the cessation of smoking. Both THC doses increased lateral position variability and the highest dose increased speed variability during curve following. Both increased headway variability, and the highest, lateral position variability during car following. Both doses caused the subjects to miss more signs indicating the need to follow another route. The high dose caused the subjects to hit the roadway obstacle more often than placebo, and also, to react slower to the subsidiary task. Yet both THC doses caused the subjects to drive in a more conservative manner. They maintained a longer headway while car following, refused morc opportunities to pass, and when they did, began this maneuver at a greater distance from the approaching vehicle. Alcohol's effects in 
this study were generally less than THC's. Chesher (1986) was puzzled by this, calling the alcohol effect "surprisingly small" and its interactive effect with THC, "unclear". Certainly it is so that BACs of $0.08 \mathrm{~g} \%$ and below have been enough to substantially degrade drivers' control of vehicular lateral position in real driving tests (Louwerens et al., 1987; Ramaekers et al., 1992a).

Stein et al. (1983) conducted two studies of alcohol and marijuana effects using a driving simulator and a 15 -minute test scenario that were very similar to those employed by Smiley et al. (1981). The former administered the two drugs in complete crossover designs. THC doses of 0,50 and $100 \mu \mathrm{g} / \mathrm{kg}$ THC were combined with BACs of 0.00 and $0.10 \mathrm{~g} \%$ in the first study. The same BACs were combined with 100 and $200 \mu \mathrm{g} / \mathrm{kg}$ in the second. This time alcohol had the expected adverse effect on practically every performance parameter, THC had little effect in the first study and little in the second in spite of the higher dose. The latter did cause the subjects to operate at generally lower speeds, however. The combination of drugs produced widely different individual reactions. After the highest THC dose, the combination produced more adverse reactions than alcohol alone.

\subsection{Actual Driving Studies}

A number of studies on marijuana's effects upon actual car driving have been reported since 1974. All studies but one were carried out on courses closed to other traffic. Klonoff (1974) conducted the exceptional study wherein 64 subjects drove on a closed course and 38 also participated in a city driving test. In his first study subjects were assigned to one of three groups that were treated with (1) placebo, (2) $4.9 \mathrm{mg}$ THC, and (3) $8.4 \mathrm{mg}$ THC. They undertook eight tests: a slalom, two tunnel tests, a funnel test, a backing up, turning in a corner, a risk judgment test and an emergency braking test. Except for the latter two, the performance measure was number of cones hit. Subjects performed 20 trials in four blocks of five. Treatments were administered between the third and fourth block and each subject's performance was related to his/her performance predicted by means of regression analysis over the first three blocks. Performance after placebo was as predicted, but after marijuana, significantly worse, though not much. The low dose impaired performance in two tests (tunnel and corner) and the high dose in five (slalom, both tunnel tests, funnel and risk judgment).

Subjects in the city driving test were divided among four groups who were treated with placebo and marijuana, on separate occasions a week apart. The respective groups' treatments were (1) placebo followed by $4.9 \mathrm{mg} \mathrm{THC}$, (2) the same in reverse order, (3) placebo followed by $8.4 \mathrm{mg} \mathrm{THC}$, and (4) the same in reverse order. After smoking a placebo or marijuana cigarette, the subjects drove for 45 minutes over a $16.8 \mathrm{mi}(27.0 \mathrm{~km})$ route on city streets while aspects of 
their performance were rated by a professional examiner using an abbreviated version of the British Columbia Department of Motor Vehicles' standard driver's licensing test. All subjects were allowed to complete the test which indicates that their performance never became dangerously unsafe under the drug's influence. Nonetheless, the examiner rated the subjects' performance as significantly worse on scales of judgement and concentration following the highest but not the lowest dose. The majority showed some impairment, but $32 \%$ after the low dose and $16 \%$ after the high dose performed significantly better than they had following placebo suggesting qualitative differences between the drug's effects in different subjects.

Hansteen et al. (1976) tested sixteen subjects in four conditions, (1) placebo alcohol + placebo marijuana, (2) placebo alcohol +marijuana (THC dose of $21 \mu \mathrm{g} / \mathrm{kg}$ ), (3) placebo alcohol + marijuana (THC dose of $88 \mu \mathrm{g} / \mathrm{kg}$ ), and (4) alcohol (BAC $0.07 \mathrm{~g} \%$ ) + placebo marijuana. Subjects were instructed to drive through a $1.1 \mathrm{mi}(0.7 \mathrm{~km})$ course delineated by traffic cones as quickly as possible but without exceeding $30 \mathrm{mph}(19 \mathrm{~km} / \mathrm{h})$. Performance was measured shortly after smoking and three hours later. Number of cones hit, 'rough handling' (superfluous and/or awkward movements as observed by an accompanying investigator), and driving time were scored. More cones were hit and more time was taken to complete each lap after consuming the higher THC dose, but no increase in rough handling was observed. Alcohol, on the other hand, adversely affected both performance measures and diminished the time taken to complete each lap. The authors concluded that the drug effects on performance were not dramatic since no major differences were found between conditions with respect to observer ratings.

Casswell (1979) was the first who included a subsidiary task to simulate the demands for monitoring the environment. Thirteen males were tested in three treatment sessions receiving alcohol and marijuana treatments twice in each session and drove for 35 minutes after each treatment. Treatments included (1a) alcohol $(0.10 \mathrm{~g} \% \mathrm{BAC})+$ placebo marijuana, (1b) placebo alcohol + marijuana (6.25 mg THC), (2a) double placebo, (2b) placebo alcohol +marijuana $(6.25 \mathrm{mg}$ THC), (3a) alcohol ( $0.05 \mathrm{~g} \% \mathrm{BAC})+$ marijuana ( $3.12 \mathrm{mg} \mathrm{THC})$, and (3b) alcohol $(0.05 \mathrm{~g} \% \mathrm{BAC})+$ marijuana $(3.12 \mathrm{mg} \mathrm{THC})$. Subjects' tasks included overtaking, driving on straight sections, through a hairpin bend, and through narrow gaps, while responding to road signals, traffic signals, and auditory signals in the subsidiary task. Alcohol alone and in combination with marijuana produced more coarse steering corrections, higher speed and increased lateral position variability. Marijuana alone was associated with lower driving speed and prolonged reaction times in the subsidiary task. Reaction times were also prolonged by the combination of marijuana and alcohol. The authors said that drivers under the influence of marijuana appeared to compensate for what they felt were the adverse effects of the drug by maintaining control effort, and decreasing speed 
to reduce the required rate of information processing. Alcohol, in contrast, appeared to produce more risky behavior.

Attwood et al. (1981) also employed normal driving tasks on a closed course. Eight males participated in a within-subjects design, receiving (1) double placebo, (2) alcohol (0.08 g\% BAC) + placebo marijuana, (3) placebo alcohol+marijuana $(150 \mu \mathrm{g} / \mathrm{kg} \mathrm{THC})$, and (4) alcohol $(0.04 \mathrm{~g} \% \mathrm{BAC})+$ marijuana $(75 \mu \mathrm{g} / \mathrm{kg} \mathrm{THC})$. The driving tasks were performed on an airfield runway and included: maintenance of a constant lateral position and velocity, maintenance of a constant headway while following a lead car that varied in speed, bringing the car to a smooth stop at a traffic signal, and deciding whether or not to overtake a preceding vehicle in the presence of an approaching car. The latter maneuver was, however, not actually undertaken. Various measures, as speed, lateral position, acceleration and headway, were taken but the number of significant comparisons were no more than expected by chance. All measures were then subjected to a discriminant analysis that separated overall treatment effects. Overall driving performances after all drug treatments were significantly worse than following placebo when tested in this multivariate analysis. Smiley (1986) suspected that the lack of univariate effects was attributable to the low number of subjects and the lack of a subsidiary task.

Peck et al. (1986) assigned 84 subjects in equal proportions to four treatment conditions: (1) double placebo, (2) alcohol ( $0.08 \mathrm{~g} \% \mathrm{BAC})+$ marijuana placebo, (3) marijuana (19 mg THC) +alcohol placebo, and (4) both drugs combined. If these subjects could have inhaled all of the drug available in the cigarette, one weighing $70 \mathrm{~kg}$ (154 lb; population average) would have received a dose of about $270 \mu \mathrm{g} / \mathrm{kg}$. Because of the remaining butt, the actual THC dose probably never exceeded $250 \mu \mathrm{g} / \mathrm{kg}$. The subjects were tested four times in complete replications of a driving test battery beginning shortly after drug administration and continuing at hourly intervals thereafter. Ratings of the subjects' driving proficiency were obtained from driving licence examiners who rode with the subjects or observed them from static positions at points along the course; and, by California Highway Patrol officers who followed the subjects' vehicle in a police car. A computerized system within the subjects' vehicle recorded their use of controls, speed and lateral position relative to course delineation. A risk acceptance test was included to measure the subjects' willingness and ability to drive through gaps wider and narrower than the vehicle. Other tasks involved stopping in response to signals, making a forced lane change and driving through pylons in a chicane. Finally, a standard police field sobriety examination and two standard laboratory tests (tracking and time estimation) were administered to the subjects outside of the vehicle. Several hundred measures of performance were obtained. No dramatic performance failures were reported as an effect of either drug or their combination. In general, the number of significant drug effects on particular 
measures were about what one might expect given the total number of statistical tests.

The investigators resorted, like Attwood et al., to multivariate statistical analysis of their data. Twelve performance measures were combined in discriminant analysis, which significantly separated the effects of each drug or their combination from placebo's. The THC effect was significant over all four replications of the tests, being greatest in the first trial. Alcohol's effect was greatest in the second trial and slightly greater than THC in every one. The combination of THC and alcohol produced significantly more impairment then did either drug alone in the first and third trials. Field sobriety checks by the police and ratings of the subjects' driving proficiency by experts failed to show any effect of THC, though these did reveal the effects of that drug in combination with alcohol. Practically the only indication of a serious effect of THC was provided by the officers following the subject's vehicle in a police car. They reported that they would have stopped the subject for suspicion of being intoxicated on $32 \%$ of all THC trials (alcohol $50 \%$, both drugs $60 \%$ ). But they also said they would have stopped $15 \%$ of the placebo treated subjects. This either indicates that the subjects were exceptionally poor drivers, or were made to appear so under conditions of the test, or that the officers were responding to cues that they ordinarily would have ignored in real driving conditions.

Smiley et al. (1987) tested the effects of marijuana $(0,100$ and $200 \mu \mathrm{g} / \mathrm{kg}$ THC) in combination with alcohol $(0.00$ and $0.05 \mathrm{~g} \% \mathrm{BAC})$ and alcohol alone $(0.08 \mathrm{~g} \%$ $\mathrm{BAC})$ on driving in a closed-course study. Treatments were administered to groups of nine males over a three hour period in a party-like atmosphere in the evening. Subjects drove shortly after smoking as well as on the following morning. Driving tasks included maintenance of a constant lateral position at $80 \mathrm{~km} / \mathrm{h}$ (50 mph), curve following, car following, route navigation, obstacle avoidance, and emergency decision making. Additionally, subjects had to perform a subsidiary task requiring visual monitoring. The high THC dose resulted in increased headway and headway variability. Alcohol alone at the $0.05 \mathrm{~g} \% \mathrm{BAC}$ level produced increased speed. Number of subsidiary task detections decreased at $0.05 \mathrm{~g} \%$ BAC but increased at $0.08 \mathrm{~g} \%$ BAC. Smiley's (1986, p. 133) conclusion from her own and previous studies was as follows:

“..., marijuana does appear to impair driving behaviour. However, this impairment is mediated in that subjects under marijuana treatment appear to perceive that they are indeed impaired. Where they can compensate, they do, for example, by not overtaking, by slowing down and by focusing their attention when they know a response will be required. Unfortunately, such compensation is not possible where events are unexpected or where continuous attention is required. Effects on driving behaviour are present shortly after smoking but do not continue for extended periods." 


\subsection{General Conclusion}

The foremost impression one gains from reviewing the literature is that no clear relationship has ever been demonstrated between marijuana smoking and either seriously impaired driving performance or the risk of accident involvement. The epidemiological evidence, as limited as it is, shows that the combination of THC and alcohol is over-represented in injured and dead drivers and more so in those who actually caused the accidents to occur. Yet there is little if any evidence to indicate that drivers who have used marijuana alone are any more likely to cause serious accidents than drug free drivers. To a large extent, the results from driving simulator and closed-course tests corroborate the epidemiological findings by indicating that THC in single inhaled doses up to $250 \mu \mathrm{g} / \mathrm{kg}$ has relatively minor effects on driving performance, certainly less than BACs in the range $0.08-0.10 \mathrm{~g} \%$. In contrast to this, laboratory studies have repeatedly shown performance impairment occurring after inhaled doses as low as about $40 \mu \mathrm{g} / \mathrm{kg}$. These became large and persistent after $100-200 \mu \mathrm{g} / \mathrm{kg}$ doses. Tracking, divided attention and vigilance test performance were particularly vulnerable to THC's effects.

It is exceedingly difficult to explain the disparity in results obtained by laboratory tests and in driving simulations. Rather than try, it seems better for the moment to assume that both sets of results are valid for the particular circumstances under which they were obtained (this issue is discussed in the General Discussion, Section 9.4.4). It demonstrates, however, that performance decrements obtained under the artificial and non-life threatening conditions in the laboratory do not automatically predict similar decrements in driving simulations that are closer to real-world driving.

This all leaves the effects of THC on actual driving performance an open question. Authors' conclusions have gone both ways. In 1977, for example, one author concluded from the literature that "cannabis is, as should have been anticipated, a hazardous drug for the road user" (Milner, 1977, p. 2), whereas another concluded that "there is no evidence that marijuana is a significant public safety problem" (McBay, 1977, p. 97). The situation was unchanged in 1985 as illustrated by the following quotations:

"It should be clear from the above review that there is more than sufficient experimental evidence to conclude that marihuana seriously impairs psychomotor performance required for driving ... Any situation in which safety both for self and others depends upon alertness and capability of control of man-machine interaction precludes the use of marihuana." (Moskowitz, 1985, p. 342) 


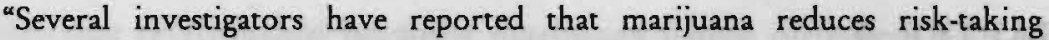
propensity and driving speed. Because of these compensating tendencies, it is presently not possible to assess the net impact of marijuana as a causal agent in traffic accidents. Although some increased risk appears likely, the magnitude of the risk remains obscure ... Many of the laboratory marijuana studies which have shown the greatest psychomotor impairment have utilized tasks that are only abstractly related to driving ... it does not necessarily follow that performance on a highly novel and complex laboratory task designed to magnify performance decrements is correlated with actual 'real world' performance in a vehicle. The fact that attempts to measure response to simulated accident situations have not consistently detected a marijuanainduced decrement, even at high dose levels, underscores the need for more research." (Peck et al., 1986, pp. 152-153)
\end{abstract}

Because epidemiological research seems too handicapped by practical constraints to demonstrate the relationship between THC and traffic accidents, and since previously applied experimental approaches provide different indications concerning the strength of that relationship, it seemed timely to investigate cannabis' effects on driving performance in the real traffic environment.

As mentioned above, only one study has been conducted in actual traffic before this program started (Klonoff, 1974). But Moskowitz (1985) and Smiley (1986) rightly criticized the method used by Klonoff for measuring driving performance on the grounds that the examiners' reliability was never determined and that the scoring instrument had never been shown to provide measures related to driving safety. Smiley questioned, for example, whether ratings of posture and irritability are relevant for good driving performance. Finally, Klonoff administered relatively low THC doses to his subjects. The effects of high doses of THC on driving in real traffic still needs to be determined.

The studies reported in this dissertation were conducted to escape these limitations. First, the effects of low, moderate and high THC doses on highway driving were determined, both in the absence and presence of other traffic. Second, Klonoff's city driving study was replicated, with some modifications with regards to the employed procedures and with the addition of another group of subjects who undertook the same driving test but then under the influence of a low dose of alcohol. The next chapter summarizes the designs and general methods for the studies that were so conducted. Thereafter, each study is reported separately in chapters that follow. 


\section{CHAPTER 4}

\section{GENERAL METHODS}

Before presenting the designs and results of the individual studies in separate chapters, it seems appropriate to describe the studies of the program and certain procedures that were common to all. In particular, compliance with ethical and legal standards, subject recruiting, marijuana cigarettes and smoking procedures, screening for the presence of other illicit drugs and alcohol, and blood sampling procedures and quantitative analyses will be elucidated.

\subsection{Description of a 4-Study Program}

The program was set up to determine the dose-response relationship between marijuana and objectively and subjectively measured aspects of real world driving; and, to determine whether it is possible to correlate driving performance impairment with plasma concentrations of the drug or a metabolite. These goals are the same as those of many unsuccessful investigations in the past. Yet none before has gone so far in seeking to achieve them in the environment where the 'drugs and driving' problem actually exists. In the present studies, a variety of driving tasks were employed, including: maintenance of a constant speed and lateral position during uninterrupted highway travel, following a leading car with varying speed on a highway, and city driving. The purpose of applying different tests was to determine whether similar changes in performance under the influence of THC occurs in all thereby indicating a general drug effect on driving safety.

The program consisted of one minor and three major studies; a series of separate but interdependent experiments that successively approached driving reality. This approach was necessary to ensure subject safety throughout the program. The program started with a laboratory study (Chapter 5), conducted in a hospital under strict medical supervision, to identify THC doses that recreational marijuana users were likely to consume before driving.

The first driving study (Chapter 6) was executed on a closed section of a public highway. The major goal was to determine the dose-response and dose- 
response-time relationship between marijuana (three different THC doses, and placebo) and road tracking precision as measured by the 'weaving' motion of the subject's vehicle during uninterrupted highway travel. Results of this are compared to those from a previous study undertaken by the investigators to measure the effects of different blood alcohol concentrations on driving performance in essentially the same test situation (Louwerens et al., 1987). A practical purpose was to determine whether the drug's effects as measured in a standard driving test were of a magnitude that would safely allow application of the same test and others on public roads in traffic.

Upon completion of this study with the demonstration that THC's effects could be safely controlled, a second driving study (Chapter 7) was conducted to come a step closer to driving reality than its predecessor. The methods applied were, with the addition of a car following test, the same as those used in the first driving study. However, driving tests were now conducted on a highway in the presence of other traffic. The greatest discretion was employed in designing this study to reach limited objectives. We choose a conservative approach which closely follows that used to determine the tolerability of medicinal drugs in human pharmacological research. It is to test THC's effects on actual driving performance in an ascending dose series. The ultimate goal was to define the THC dose (or plasma concentration) limit which separates low and high risk driving performance impairment by approaching it from the bottom up.

Yet normal driving is far more complex and varied than simply to maintain a safe lateral position and headway during uninterrupted travel on a highway. A THC dose having no effect on these parameters might still impair driving performance in more complex urban driving situations. For this reason the program then proceeded into the third and final driving study (Chapter 8) which involved tests conducted in high density urban traffic. The highest dose which had no significant effect on highway driving in the previous study was given to subjects who would now operate in an urban driving test. This provided an opportunity to measure a far broader range of driving performance. If no effect were again observed, the generality of the dose-effect relationship would be strengthened. But if a new kind of impairment were observed, the conclusion would have to be that the dose effect relationship can not be validly used to define the effects of THC on driving performance, in general. The nature of the new impairment would provide insight into the kinds of traffic safety problems that may be first to appear as a consequence of the drug's effects. A second group also participated in this study and undertook the same driving test, but then after drinking alcohol (reaching an average BAC of $0.04 \mathrm{~g} \%$ ), and a placebo. This was done for two reasons; first, the alcohol condition served as a control whether the employed tools to assess driving performance were sensitive; and, secondly, it made a comparison possible between low doses of alcohol and THC. 


\subsection{Compliance with Ethical and Legal Standards}

All studies described in this report complied with the code of ethics on human experimentation established by the Declaration of Helsinki (1964) as amended in Tokyo (1975), Venice (1983), and Hong Kong (1989). This implies that the volunteer subjects were fully informed of all procedures, possible adverse reactions to drug treatments, legal rights and responsibilities, expected benefits of a general scientific nature, and their right for voluntary termination without penalty or censure. All subjects gave their informed consent, in writing. Their anonymity was and will be maintained in all communications from the project. The investigators provided for continuous medical supervision and emergency medical treatment during the studies. Approvals for individual studies were separately obtained from the University's Medical Ethics Committee.

Before the program started an Independent Advisory Committee was formed whose function was to ensure that the program proceeded in accordance with all medical and legal standards. This committee comprised the Assistant District Attorney, the Municipal Traffic Attorney for the City of Maastricht, a member of the University's Medical Ethics Committee, and the Dutch Regional Inspector for Public Health (Drugs). A permit for obtaining, storing and administering marijuana was obtained from the Dutch Drug Enforcement Administration.

Subjects were accompanied on every driving test by a licensed driving instructor experienced in supervising subjects who operated under the influence of medicinal drugs in previous studies. The instructor's sole task was that of monitoring ride safety. Redundant control system in the test vehicle was available for controlling the car if emergency situations should arise. However, the primary guarantor of the subject's safety was the subject himself/herself. The subject, like any licensed Dutch driver, had the legal responsibility to stop driving when feeling 'under the influence' to the point where he/she could no longer be sure of his/her ability for safely controlling the vehicle. Subjects in this investigation were reminded of their responsibility and urged not to undertake any test, or to stop driving during a test in progress, if they felt incapable of driving safely. Subjects were always transported to and from their appointments and were strictly instructed not to operate their own vehicles for a period of 12 hours after having received the experimental treatment.

\subsection{Subject Recruiting}

The ideal subjects would be male and female marijuana users whose consumption of the drug represents that of the majority in that particular population. Van der Wal's (1985) data for the oldest group (17-18 years) in his sample of present Dutch cannabis users indicate that about $56 \%$ of the males and females have a 
usage frequency of more than once per month and less than daily. This usage frequency was considered as the first selection criterion.

The second criterion was that the users should also be experienced drivers in possession of a driver's licence. Subjects must have driven at least $5,000 \mathrm{~km}$ $(3,108 \mathrm{mi})$ per year over the previous three years. This criterion was, however, not always met because of the difficulties in recruiting subjects.

As the third criterion the users should have indicated on a questionnaire that they had driven within one hour after smoking cannabis at least once within the preceding year. These users not only possess the requisite driving experience under the influence of marijuana, they also constitute the 'drivers at risk'. In addition, the application of this criterion avoided the ethical dilemma of requiring subjects to accept a risk which they would otherwise avoid.

As a fourth criterion, the subjects should agree to refrain from their normal marijuana use for at least five days prior to their participation in any test.

Other inclusion criteria were as follows: age 21-40 years; normal (corrected or uncorrected) binocular acuity (i.e. 20/25 Snellen acuity, or better); body weight within the $85^{\text {th }}-115^{\text {th }}$ percentile range according to the 1983 table from the Metropolitan Life Insurance Company; and, Dutch nationality. The latter criterion was a condition set by the Dutch Ministry of Health which has no authority to permit the use of an illicit drug by foreign nationals.

Exclusion criteria included the following:

1. No history of treatment for drug or alcohol abuse or addiction and no reasonable possibility of dependence occurring as the result of participation in the investigation.

2. No record of arrests or conviction for drug trafficking.

3. No history of psychiatric or organic brain disorders.

4. No overt signs of cardiovascular, respiratory, renal, hepatic, metabolic or neuromuscular disorders and no history of serious disorders of this type.

5. No current use of any psychoactive medication (tranquilizers, antidepressants etc.)

6. For females, no pregnancy or any reasonable probability that pregnancy might occur during participation in the investigation.

Some subjects volunteered spontaneously after reading about the planned study in newspapers. Other volunteers for the first two studies were primarily obtained from among the local population of marijuana users by means of advertisements. Both the second and the third driving study required new samples of subjects. In these cases it was more difficult to recruit subjects since advertisements could not be placed where they might attract the attention of news media. The desire to avoid attention was fostered by a need to ensure subjects' anonymity and avoid the media's interference with data collection involving driving in traffic on public highways and city streets. Subjects were therefore recruited in the last two studies mainly by contacts obtained from subjects from the preceding ones. Admittedly 
this procedure is not the best to acquire independent samples but was necessary for practical reasons.

Volunteers were screened in two stages; first from their responses to a combined cannabis use, driving experience and medical history questionnaire; and secondly, on the basis of an interview and physical examination. Furthermore law enforcement authorities were contacted, with the volunteers' consent, to verify that they had no previous arrests or convictions for drug trafficking.

Subjects were instructed to sleep normally on the nights before test days. Alcohol consumption was prohibited for 24 hours before tests, and consumption of beverages containing caffeine, for 2 hours beforehand. Those who smoked tobacco were advised that this would also be prohibited for one hour before testing until its completion.

\subsection{Marijuana Cigarettes and Smoking Procedures}

Marijuana cigarettes were supplied by the U.S. National Institute on Drug Abuse. They were manufactured, dried, and analyzed for cannabinoid content at the U.S. Research Triangle Institute from Mexican marijuana grown by the University of Mississippi. Placebo cigarettes were prepared by ethanol extraction of cannabinoids from the plant stock. Cigarettes looked like standard tobacco cigarettes: they were approximately $85 \mathrm{~mm}$ in length $\mathrm{x} 25 \mathrm{~mm}$ in circumference and weighed an average of about $800 \mathrm{mg}$. Cigarettes were stored at $-20^{\circ} \mathrm{C}$ and their moisture content was raised to about $16 \%$ for moderating their 'harshness' by humidification at room temperature over saturated sodium chloride solution in a closed container over the night before smoking.

Subjects smoked the administered cigarettes through a plastic holder in their customary fashion. In the laboratory study, they were allowed to smoke part or all of the THC content in three marijuana cigarettes, but with the constraint that smoking had to be finished within fifteen minutes. In the subsequent driving studies, cigarettes were cut to different lengths to provide the THC doses appropriate for the individuals' body weights, and subjects were encouraged to smoke the entire dose in five to ten minutes. The THC concentrations of the administered marijuana cigarettes varied between 1.75 and $3.58 \%$, and are mentioned in the 'Methods' of the respective studies.

After cessation of smoking, cigarettes were carefully extinguished and retained for subsequent gravimetric estimation of THC consumed (i.e. the difference between the weight of the original cigarette and the remaining unsmoked portion times the THC proportion in the original cigarette). This method of estimating THC amounts consumed is based upon the assumption that THC is equally distributed over the entire cigarette. Perez-Reyes et al. (1982) analyzed THC concentrations in the unsmoked portions of marijuana cigarettes of three 
different potencies and indeed found that they were identical to those in the unlit cigarette.

\subsection{Screening for the Presence of Other Illicit Drugs and Alcohol}

Though it seemed unlikely that subjects would regularly resort to using other illicit drugs or alcohol prior to controlled marijuana smoking and testing, the possibility could not be definitely excluded without testing the subjects for the presence of these drugs. Therefore they were informed beforehand of the intention to obtain urine and breath samples which would be analyzed for the presence of prohibited agents.

Each subject was required to submit a urine sample immediately upon arrival at the test site. Samples were later assayed qualitatively for the following drugs (or metabolites): cannabinoids, benzodiazepines, opiates, cocaine, amphetamines and barbiturates. In addition a breath sample was analyzed on the spot for the presence of alcohol using a Lion S-D3 Breath-Alcohol Analyzer. The urine and breath sample screening procedures were employed in all studies in the program.

Drugs other than cannabinoids were found in urine of four subjects. In the laboratory study, the urine of two subjects was positive for benzodiazepines; and, of one subject for barbiturates. Analyses of six urine samples obtained from these subjects during the successive driving study failed to show the presence of these drugs. Since all urine samples from both the laboratory and first driving study were analyzed after completion of the latter, the failure to detect the drugs in samples obtained during the driving study indicates that they did not abuse these drugs. Upon questioning, all three subjects denied that they had taken these drugs. Since no urine or plasma was left from these subjects, it was, however, not possible to check whether the results were false positives. Data obtained from these subjects in the laboratory study were not excluded from the statistical analyses. One subject's urine, obtained prior to smoking in the $200 \mu \mathrm{g} / \mathrm{kg}$ condition in the first driving study, was positive for cocaine. Upon questioning, the subject replied that some friends had surreptitiously administered him cannabis cake and cocaine the day before. Assuming that the drugs' effects had dissipated the next day, these subject's data were also not excluded from statistical analyses.

\subsection{Blood Sampling and Quantitative Analyses}

Blood samples were taken by venepuncture. Two $10 \mathrm{ml}$ aliquots were obtained in every case. These were heparinized and centrifuged within 30 minutes. Plasma was placed in frozen $\left(-20^{\circ} \mathrm{C}\right)$ storage prior to analysis. The quantitative chemical 
analysis of THC and THC-COOH in plasma was performed by gas chromatography/mass spectrometry (GC/MS) using deuterated cannabinoids as internal standards (Möller et al., 1992). Of the many analytic techniques available at present, GC/MS is the reference method of choice (Cook, 1986). Applying this method, the detection limits for THC and THC-COOH were about 0.3 and $3.0 \mathrm{ng} / \mathrm{ml}$, respectively. THC and $\mathrm{THC}-\mathrm{COOH}$ concentrations in plasma will further be abbreviated to [THC] and [THC-COOH].

If the urine analysis (above) was positive for cannabinoids, plasma taken before smoking was also analyzed to quantitatively determine [THC] and [THC-COOH]. Subjects with detectable THC in pre-smoking plasma are shown in Table 4.1. In the laboratory and first driving study, THC was detected in each pre-smoking plasma sample from two subjects and in one sample from another subject, namely prior to smoking in the $200 \mu \mathrm{g} / \mathrm{kg}$ condition. In the second driving study, THC was detected in one sample from one male and in five out of six samples from another male. In the city driving study, THC was not detected in any pre-smoking sample.

Table 4.1 Pre-smoking and post-smoking [THC] in subjects with detectable THC in pre-smoking plasma samples.

\begin{tabular}{|c|c|c|c|c|}
\hline \multirow[b]{2}{*}{ Study } & \multirow[b]{2}{*}{ Subject no. } & \multirow[b]{2}{*}{ Condition } & \multicolumn{2}{|l|}{$[\mathrm{THC}](\mathrm{ng} / \mathrm{ml})$} \\
\hline & & & Before Smoking & After Smoking ${ }^{* *}$ \\
\hline \multirow[t]{2}{*}{ laboratory study } & 1513 & )$^{*}$ & 3.0 & 35.5 \\
\hline & 1525 & $r^{*}$ & 1.2 & 13.3 \\
\hline \multirow[t]{9}{*}{$1^{\text {" driving study }}$} & 1507 & $200 \mu \mathrm{g} / \mathrm{kg}$ & 1.9 & 39.3 \\
\hline & 1513 & placebo & 2.0 & 1.8 \\
\hline & & $100 \mu \mathrm{g} / \mathrm{kg}$ & 2.4 & 9.2 \\
\hline & & $200 \mu \mathrm{g} / \mathrm{kg}$ & 2.7 & 34.7 \\
\hline & & $300 \mu \mathrm{g} / \mathrm{kg}$ & 4.2 & 26.8 \\
\hline & 1525 & placebo & 1.1 & 1.1 \\
\hline & & $100 \mu \mathrm{g} / \mathrm{kg}$ & 1.5 & 19.5 \\
\hline & & $200 \mu \mathrm{g} / \mathrm{kg}$ & 1.1 & 6.9 \\
\hline & & $300 \mu \mathrm{g} / \mathrm{kg}$ & 2.7 & 13.9 \\
\hline \multirow[t]{6}{*}{$2^{\text {nd }}$ driving study } & 1536 & $1^{\text {st }}$ placebo & 0.5 & 0.6 \\
\hline & & $100 \mu \mathrm{g} / \mathrm{kg}$ & 0.6 & 10.7 \\
\hline & & $2^{\text {nd }}$ placebo & 0.7 & 0.6 \\
\hline & & $3^{\text {rd }}$ placebo & 1.2 & 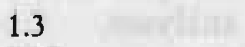 \\
\hline & & $300 \mu \mathrm{g} / \mathrm{kg}$ & 1.3 & 30.9 \\
\hline & 1537 & $1^{\text {st }}$ placebo & 0.6 & 0.5 \\
\hline
\end{tabular}

1. only one condition (smoking until the desired effect was achieved).

i.. sampling time was 40,30 and 35 minutes after initiation of smoking in the laboratory, first and second driving study, respectively. 
It seems obvious that those subjects, who had detectable [THC] before smoking, did not comply with the instruction to abstain from cannabis consumption for at least five days prior to the trial. They all had long histories (at least 7 years) of cannabis experience and were frequent (at least twice a week) users. Gieringer (1988) reports that THC may persist in the blood of chronic smokers at levels up to $4.0 \mathrm{ng} / \mathrm{ml}$ after 48 hours. It therefore remains an open question when their latest consumption was or whether they were impaired upon arrival at the laboratory.

The same pattern of pre- to post-smoking values as shown in Table 4.1 was observed in the other subjects, i.e. [THC] and [ $\mathrm{THC}-\mathrm{COOH}]$ increased considerably after smoking the administered marijuana cigarettes and not following placebo. Therefore, these subjects' data were not excluded from the statistical analyses.

Fifty percent of the pre-smoking plasma samples obtained from subjects in the laboratory and first driving study, whose urine tests were negative for cannabinoids, were also analyzed. These analyses were performed to examine whether any false negative urine analyses had occurred. Results showed that none of these samples contained detectable [THC] or [THC- $\mathrm{COOH}]$. From these results it was inferred that in subsequent studies pre-smoking blood samples need only be taken if the urine test for cannabinoids were positive. 


\section{CHAPTER 5}

\section{LABORATORY STUDY TO SELECT THC DOSES}

\subsection{Introduction}

Doses used in all previous studies of inhaled THC have been selected without consulting the subjects beforehand to determine whether these realistically approximated doses they commonly use. In the opinion of several experts (Moskowitz, 1985; Chesher, 1986; Peck et al., 1986) this has resulted in the selection of maximum doses for experimental purposes that are considerably less than those used for recreational pursuits. One could arbitrarily select higher doses but with the risk of erring in the opposite direction. A dose of, say $300 \mu \mathrm{g} / \mathrm{kg}$, might be higher than any taken at one time by street users or, at any rate, higher than one they might take and then drive. If we were to select unrealistically high doses these could result in bizarre and potentially dangerous reactions in even the most controlled driving test. To avoid arbitrarily selecting the wrong maximum dose, it seemed necessary to consult the subjects in the context of a 'clinical' study.

The laboratory study's major purpose was therefore to establish the maximum dose for subsequent driving studies. Yet it provided several opportunities for obtaining valuable information about THC's pharmacokinetics and its pharmacodynamic effects after marijuana smoking. Blood samples were repeatedly taken for measuring [THC] and [THC-COOH]. The subjects repeatedly performed certain simple laboratory tests, estimated their levels of intoxication and indicated their willingness to drive under several specified conditions of urgency. In addition, heart rate was measured at these times. The secondary purpose was that of specifying relationships between [THC] and [THC-COOH] with changes in the other physiological, performance or subjective variables.

\subsection{Methods}

Subjects

Twenty-four healthy volunteers, 12 males and 12 females, volunteered to 
participate in this study. They were screened as described in Chapter 4. Groups of six were treated and tested per night. Sessions were conducted in the evening between 19.00 and 24.00 hours and subjects smoked and were tested at staggered intervals of 10 minutes. GC/MS analysis of plasma obtained from one male revealed trivial amounts of values THC in the first and second samples after smoking but none thereafter, and no THC-COOH in any sample. It was concluded that this subject had not inhaled smoke so his data were excluded from further analyses. Characteristics of the remaining 23 subjects are given in Table 5.1. T-tests for independent samples showed that males were more experienced smokers than females $(p<.044)$. Males' driving experience was nearly significantly greater than females' $(p<.056 \& .089$ for number of years and $\mathrm{km}(\mathrm{mi})$, respectively).

Table 5.1 Mean \pm SD (range) of subjects' characteristics.

\begin{tabular}{lll}
\hline & $\begin{array}{l}\text { males } \\
(\mathrm{N}=11)\end{array}$ & $\begin{array}{l}\text { females } \\
(\mathrm{N}=12)\end{array}$ \\
\hline age $(\mathrm{yrs})$ & $27.0 \pm 4.6(22-38)$ & $24.6 \pm 2.9(21-31)$ \\
weight $(\mathrm{kg})$ & $69.8 \pm 10.3(56.5-87.0)$ & $66.6 \pm 9.1(52.0-79.0)$ \\
weight $(\mathrm{lb})$ & $154 \pm 23(125-192)$ & $147 \pm 20(115-174)$ \\
smoking experience (yrs) & $9.2 \pm 5.8(1-23)$ & $5.0 \pm 3.3(2-14)$ \\
\# joints/month & $5.9 \pm 3.7(1-14)$ & $5.8 \pm 5.4(1-20)$ \\
driving experience (yrs) & $8.5 \pm 5.7(3-23)$ & $4.8 \pm 2.7(1-12)$ \\
driving experience (km x 1000) & $121 \pm 118(15-360)$ & $54 \pm 54(5-175)$ \\
driving experience (mi x 1000) & $75 \pm 73(9-224)$ & $34 \pm 34(3-109)$ \\
\# of subjects having driven within & & \\
1 hr following cannabis consumption: & & 9 \\
less than 5 times & 1 & 3 \\
5 times or more & 10 & \\
\hline
\end{tabular}

\section{Marijuana Cigarettes and Smoking Procedure}

The marijuana cigarettes had an average weight of $767 \mathrm{mg}$ and contained $2.57 \%$ or about $20 \mathrm{mg}$ THC. The subjects were allowed to smoke part or all of the THC content in three cigarettes until achieving the desired psychological effect. Cigarettes were smoked ad lib through a plastic holder. The only requirement was to smoke continuously for a period not exceeding fifteen minutes. When subjects voluntarily stopped smoking, cigarettes were carefully extinguished and retained for subsequent gravimetric estimation of THC consumed.

\section{Measurements}

A test battery which lasted 20 minutes took place before smoking and was repeatedly administered at $30,90,150$ and 210 minutes after initiation of smoking. The battery consisted of: 
1. The Critical Tracking Test (CTT). This test, described in detail by Jex et al. (1966), was implemented on a IBM-compatible MS-DOS computer and measures the subject's ability to control a displayed error signal in a compensatory tracking task which increases in difficulty during a trial. Error appears as horizontal deviation of a cursor from midpoint on a horizontal, linear scale. Compensatory joy-stick movements null the error by returning the cursor to the midpoint. As the level of instability of the cursor increases during the trial, in direction and speed, the task becomes more difficult and eventually the subject is unable to compensate. The level of instability at which the subject loses control, called the critical frequency or $\lambda_{c}$, is expressed in angular change of radius per second $(\mathrm{rad} / \mathrm{s})$. Theoretically, $\lambda_{c}$ is the reciprocal of the operating delay lag in human closed-loop manual control. The test included 5 trials of which the lowest and highest score were removed; the average of the remaining scores was taken as the final test score. Total test time duration was approximately 5 minutes.

2. Questionnaires. Subjects were required to rate their feeling of 'high' as a percentage of the maximum ever experienced, and to indicate certain feelings of present cognitive and emotional state using the 16-item visual-analog scale developed and standardized for drug research by Bond and Lader (1974). Scores on the latter scales were grouped to form three cluster scores for measuring the corresponding factors: alertness, contentedness and calmness which will be expressed as percentage of the maximum. In addition, the subjects' willingness to operate a motor vehicle was assessed by asking them to declare whether they would attempt to drive for a set distance if the reasons were: $A$. unimportant though gratifying, such as for transporting a friend to another party; $B$. important but avoidable, such as for transporting a mildly sick friend home when he would otherwise have to call a taxi; and C. urgent, such as transporting a severely sick infant to the hospital. These ratings were made by subjects immediately after termination of smoking and after conclusion of the CTT.

3. Heart Rate. Heart rate was measured manually by counting the number of beats per minute immediately after completion of the questionnaires.

4. Hand Steadiness Test. Thereafter, hand steadiness was measured from the number of side contacts occurring as the subject attempted to hold a $1 \mathrm{~mm}$ (0.04 in) stylus for 15 seconds within each of five circular holes with successively diminishing diameters $(3.90,3.05,2.70,2.20$ and $1.85 \mathrm{~mm}$, respectively; or, $0.154,0.120,0.106,0.087$ and $0.073 \mathrm{in})$. Subjects were allowed to rest their hand on the table. The test score was defined as the total number of contacts of the stylus with any side. Since the distribution of subjects' scores were skewed, a square root transformation was applied to normalize data. The test lasted about 3 minutes. 
5. Blood Sampling. A blood sample was taken by means of a venepuncture ten minutes after the beginning of the test battery. The sample (2 aliquots containing $10 \mathrm{ml}$ blood apiece) was heparinized and centrifuged, and plasma was placed in frozen $\left(-20^{\circ} \mathrm{C}\right)$ storage prior to analysis for THC and its major metabolite THC-COOH. Blood samples were taken before and at 40,100, 160 and 220 minutes after initiation smoking.

Subjects were familiarized with the questionnaires and practiced the CTT and hand steadiness test on three separate occasions during the weeks prior to the test night until they reached a steady performance level.

\section{Data Analysis}

Parametric data were analyzed as follows. All data including baseline values entered a repeated measures multivariate analysis of variance (MANOVA) with Sex as a between-groups and Time as a within-subjects factor and the criterion for significance set at .05. If a significant Time effect was found, a repeated measures univariate analysis of variance (ANOVA) was performed to separately compare each post-smoking measurement with baseline. Sex was not a factor in these ANOVA's unless MANOVA had revealed a significant Sex by Time interaction. Individual comparisons with baseline were not possible for perceived 'high' ratings and plasma levels of the drug since baseline values were zero in most cases. Instead, if MANOVA revealed a significant Time effect, the data were further analyzed in an ANOVA trends analysis to determine the significance of linear, quadratic and cubic components. In the figures, the mean of the variable is depicted by a point and its standard error (SE) by the height of the vertical line above the point.

The subjects' expressions of willingness to drive were made on the basis of a dichotomous decision and could not for that reason be analyzed in the same manner as other variables. These data were therefore analyzed using Cochran's Q-statistic to determine if the proportion of subjects willing to drive changed over time. If they did significantly, separate changes from baseline were tested by McNemar's sign-test.

Criterion for statistical significance in individual comparisons was adjusted by means of the 'Sequential Bonferroni' correction (Overall and Rhoades, 1987) to retain a constant type I error probability of .05 across the entire set of comparisons. This means that for the largest of four differences tested at once, $p$ had to be less than $.05 / 4=.013$ to be judged significant. For the second, it had to be $<.05 / 3=.017$; for the third, $<.05 / 2=.025$; and for the smallest difference, $p<.05$.

Significant Sex effects were generally absent. Results pertaining to differences between the sexes are therefore only reported for the exceptional cases where the differences were significant. 
Two types of correlations were calculated to determine the linear relationship between two variables; i.e., the inter-subject and intra-subject correlation. The first is the most commonly used; it is obtained from pairs of variables measured in a group of subjects. In this study, for example, the correlation between [THC] and $\lambda_{c}$, the tracking performance score, was calculated for all subjects at each sampling time separately. This resulted in four correlations (one at $t=40,100,160$ and $220 \mathrm{~min}$ ) obtained from 23 subjects $(\mathrm{N}=23)$. These correlations were tested for significant departures from zero by $\mathrm{t}$-test.

The intra-subject correlation, on the other hand, is the correlation between pairs of variables within one subject. In the present study, for example, the correlation between [THC] and $\lambda_{c}$ was also calculated for each subject, across all sampling times, separately. This resulted in 23 correlations (one from each subject) obtained from four repeated measurements $(N=4$; baseline values were excluded). These were transformed into Fisher's $z$-scores and then averaged across

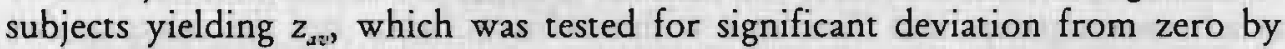
t-test, and transformed back to $r$.

Interpretations of these two types of correlation are not the same. If, in the present study, a perfect inter-subject correlation $(r= \pm 1.0)$ between [THC] and $\lambda_{c}$ existed, it would mean that one can perfectly predict $\lambda_{c}$ of a particular subject from the knowledge of his/her [THC]. Usually, however, inter-subject correlations are much lower; and, the closer to zero, the more unreliable the predictions become.

A high average intra-subject correlation means that, on the average, scores on two variables are closely related within a subject, but not necessarily between subjects. Thus, a highly negative average intra-subject correlation between [THC] and $\lambda_{c}$ (lower scores indicating poorer performance) would mean that, within a subject, higher plasma levels of THC are associated with poorer tracking performance. Yet this does not imply that, if two subjects are compared, the one with the higher plasma levels performs worse. That would only be the case if both the average intrasubject and the intersubject correlations were strongly negative.

\subsection{Results}

\section{Consumed THC}

Six subjects consumed one cigarette, thirteen smoked two and four smoked three. Total THC amounts consumed are given in Table 5.2. It should be noted that these amounts of THC represent both the amount inhaled and the portion that was lost through pyrolysis and side-stream smoke during the smoking process. Males consumed slightly more THC than females but the difference was not significant. 
Subjects were next classified into two categories according to the frequency of reported cannabis consumption. Eleven subjects were infrequent users; i.e., between once weekly to once monthly; twelve subjects were classified as frequent users; i.e. at least once weekly but less than daily. Statistical analyses failed to reveal a significant difference between the two groups.

Table 5.2 Mean, median and range of amounts of THC consumed, both in absolute values and relative to bodyweight.

\begin{tabular}{|c|c|c|c|c|c|c|}
\hline \multirow{3}{*}{$\begin{array}{l}1 \\
y \\
x=20\end{array}$} & \multicolumn{6}{|c|}{ amounts of THC consumed } \\
\hline & \multicolumn{3}{|c|}{ absolute amount (mg) } & \multicolumn{3}{|c|}{ relative to bodyweight $(\mu \mathrm{g} / \mathrm{kg})$} \\
\hline & mean & median & range & mean & median & range \\
\hline males $(\mathrm{N}=11)$ & 22.3 & 18.6 & $14.7-35.2$ & 324 & 292 & $203-524$ \\
\hline females $(N=12)$ & 19.4 & 18.9 & $11.3-28.2$ & 293 & 292 & $194-440$ \\
\hline all $(\mathrm{N}=23)$ & 20.8 & 18.8 & $11.3-35.2$ & 308 & 292 & $194-524$ \\
\hline
\end{tabular}

\section{Plasma Concentrations of the Drug}

Mean, median and range of [THC] and [ $\mathrm{THC}-\mathrm{COOH}$ ] at each sampling time are shown in Tables 5.3 and 5.4. Maximum [THC] was found in the first sample after smoking at $t=40$ minutes. Males had somewhat higher [THC] and much higher [THC-COOH] values than females; the difference between both sexes was however rather constant over time, except for [THC] at $t=40$ where the difference was quite profound. These observations were confirmed by MANOVA that showed a significant Sex effect for both [THC] and [THC-COOH] $\left(\mathrm{F}_{1,21}=4.3 \& 9.79 ; p<.05\right.$ $\& .005$, respectively), but no significant Time by Sex interaction. Since consumed THC amount did not differ between both sexes, the conclusion must either be that males were more efficient smokers than females, or that they absorbed the active ingredient differently.

There was a significant Time effect for both [THC] and [THC-COOH] $\left(\mathrm{F}_{3,19}=14.79 \& 11.70\right.$, respectively; $p<.001$ in both cases). Univariate trend analysis revealed that both linear, quadratic as well as cubic functions fitted the trend in [THC] over time significantly $\left(\mathrm{F}_{1,21}=44.56,38.95 \& 29.23 ; p<.001, .001\right.$ $\& \approx .001 ; p_{c}=.017, .025 \& .05$, respectively) due to a rapid decline of [THC] in plasma after the first sample. THC-COOH changes over time were only significantly fitted by a linear trend $\left(F_{1,21}=26.92 p<.001 ; p_{c}=.017\right)$.

The relation between consumed THC, relative to body weight, and [THC] was examined by calculation of inter-subject correlations (intra-subject correlations could not be determined from the data because each subject smoked only one dose of THC). These analyses showed moderate inter-subject correlations between both parameters at each sampling time, namely $0.42(p<.05), 0.34$ (ns), 0.42 $(p<.05)$ and $0.45(p<.05)$. Yet inspection revealed that the apparent strength of 
these correlations was almost totally attributable to two males who had consumed the greatest amounts of THC (486 and $524 \mu \mathrm{g} / \mathrm{kg}$ ) and had also very high plasma levels of THC ( 45.9 and $35.5 \mathrm{ng} / \mathrm{ml}$, respectively). Indeed, Spearman's rank correlations were small $(<0.10)$ and not significant. There were no differences between males and females with respect to these correlations.

Table 5.3 Mean, median and range of $[\mathrm{THC}]$ in $\mathrm{ng} / \mathrm{ml}$.

\begin{tabular}{llllll}
\hline & & $t=40$ & $t=100$ & $t=160$ & $t=220$ \\
\hline \multirow{2}{*}{ males $(\mathrm{N}=11)$} & mean & 17.7 & 5.8 & 2.8 & 1.7 \\
& median & 13.3 & 4.5 & 2.3 & 1.3 \\
& range & $6.7-45.9$ & $2.5-15.2$ & $1.2-6.8$ & $0.7-5.1$ \\
& & & & & \\
females $(\mathrm{N}=12)$ & mean & 9.9 & 3.0 & 1.4 & 0.7 \\
& median & 7.0 & 2.7 & 1.0 & 0.6 \\
& range & $3.3-19.3$ & $0.3-6.8$ & $0.5-3.2$ & $0.0-1.8$ \\
& & & & & \\
all $(\mathrm{N}=23)$ & mean & 13.6 & 4.3 & 2.1 & 1.2 \\
& median & 9.9 & 3.5 & 1.4 & 0.8 \\
& range & $3.3-45.9$ & $0.3-15.2$ & $0.5-6.8$ & $0.0-5.1$ \\
\hline
\end{tabular}

Table 5.4 Mean, median and range of [THC-COOH] [THC-COOH] in $\mathrm{ng} / \mathrm{ml}$.

\begin{tabular}{llllll}
\hline & & $t=40$ & $t=100$ & $t=160$ & $t=220$ \\
\hline \multirow{3}{*}{ males $(\mathrm{N}=11)$} & mean & 33.9 & 28.1 & 25.3 & 20.8 \\
& median & 25.6 & 19.6 & 18.3 & 13.5 \\
& range & $12.9-96.4$ & $12.8-72.4$ & $9.5-63.5$ & $8.0-67.0$ \\
& & & & & \\
females $(\mathrm{N}=12)$ & mean & 12.4 & 9.5 & 8.3 & 5.6 \\
& median & 9.6 & 8.2 & 7.5 & 5.5 \\
& range & $3.3-39.9$ & $0.5-26.8$ & $3.0-15.8$ & $0.0-13.0$ \\
& & & & & \\
all $(\mathrm{N}=23)$ & mean & 22.7 & 18.4 & 16.4 & 12.8 \\
& median & 17.3 & 13.9 & 13.6 & 10.1 \\
& range & $3.3-96.4$ & $0.5-72.4$ & $3.0-63.5$ & $0.0-67.0$ \\
\hline
\end{tabular}

These results indicate that the between-subject variability in [THC] is not related to the between-subject variability in the consumed amount of THC; in other words, information about [THC] and the time of blood sampling after smoking, of a particular individual, does not reveal how much that subject smoked, nor vice versa. 
Perceived ' high'

Mean subjective ratings of 'high' are shown in Figure 5.1. The subjects consistently reported their peak subjective reaction as being about $70 \%$ of the greatest ever experienced. This was achieved shortly after smoking. Their subjective feelings declined, again in a highly consistent manner between subjects, until arriving at a level less than $15 \%$ of the greatest ever experienced, at a time $31 / 4$ hours after smoking.

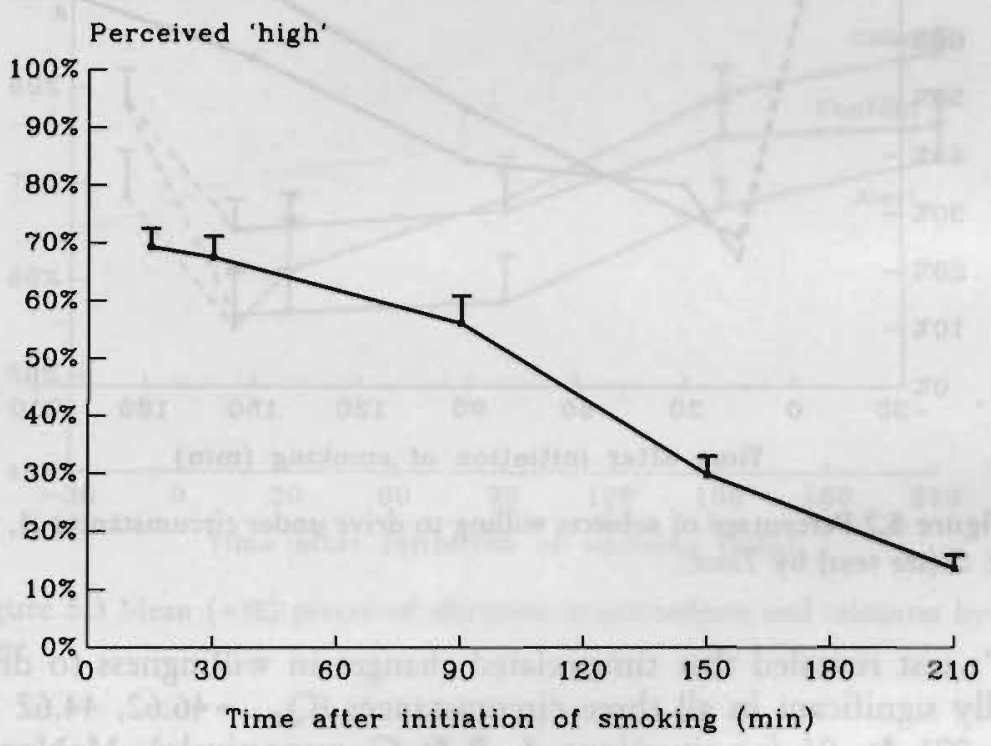

Figure 5.1 Mean (+SE) perceived 'high' by Time.

MANOVA revealed a significant Time effect $\left(\mathrm{F}_{4,18}=49.18 ; p<.001\right)$. Trend analysis confirmed the impression from the data that the downward trend is best fitted by a linear function $\left(\mathrm{F}_{1,21}=201.92 ; p<.001\right)$.

\section{Willingness to Drive}

Figure 5.2 displays percentages of the subjects that declared to be willing to drive under different circumstances of a gradually more compelling nature $(A$. unimportant though gratifying; $B$. important but avoidable; and, $C$. urgent). Only about $20 \%$ of the subjects declared their willingness to drive for relatively unimportant reasons $(A$ and $B)$, but approximately $80 \%$ declared that they would do so for an urgent reason (C), while experiencing the peak subjective reaction. Willingness to drive gradually rose to about $70 \%$ for unimportant reasons, and $100 \%$ for an urgent reason, by the end of the test session. 


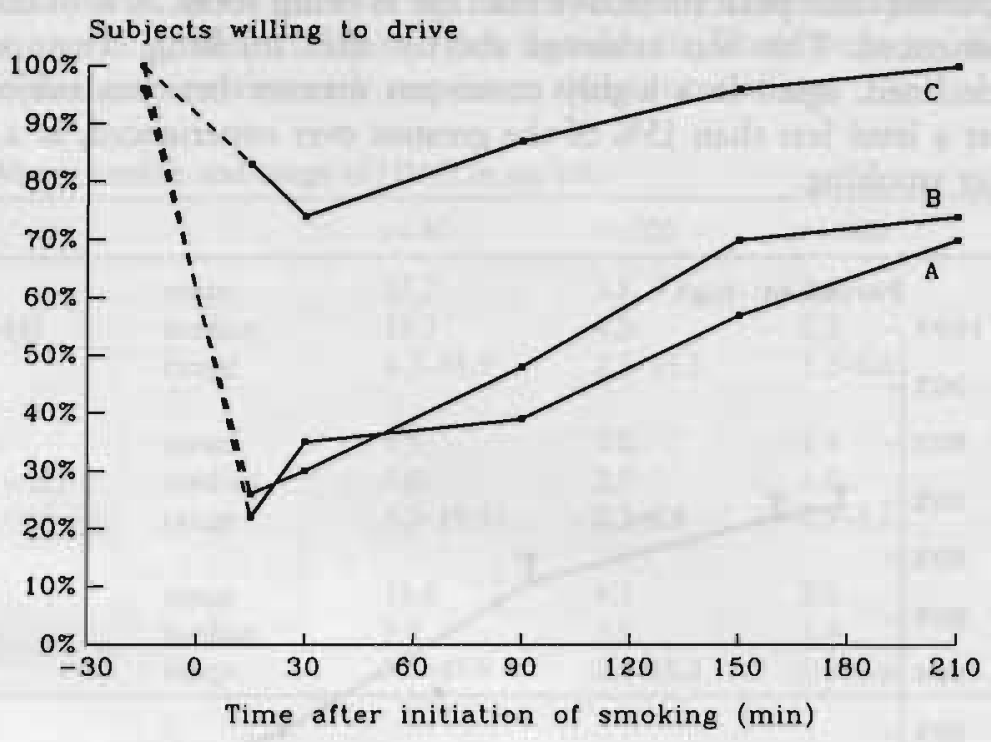

Figure 5.2 Percentage of subjects willing to drive under circumstances $A, B$ \& $C$ (see text) by Time.

Cochran's test revealed that time-related changes in willingness to drive were statistically significant in all three circumstances $\left(Q_{\mathrm{df}-5}=46.62,44.62 \& 15.17\right.$; $p<.001, .001 \& .01$ for situations $A, B \& C$, respectively). McNemar's test showed that the percentage of subjects willing to drive under circumstance $A$ was significantly different from baseline until the end of the test session $(p<.001$, $\left..001, .001, .002 \& .02 ; p_{i}=.01, .013, .017, .025 \& .05\right)$, while under circumstance $B$ changes were only significant until $2 \frac{1}{2}$ hours after smoking $(p<.001, .001 \&$ $.004 ; p_{c}=.01, .013 \&$ \& .017). McNemar's test failed to detect any significant change after smoking relative to baseline for circumstance $C$.

\section{Perceived Alertness, Contentedness and Calmness}

Figure 5.3 shows subjects' ratings of perceived alertness, contentedness and calmness. Scores on these factors changed significantly over time $\left(\mathrm{F}_{5,17}=8.19,5.79\right.$ $\& 7.02 ; p<.001, .003 \& .001$, respectively). Subjects felt significantly less alert relative to baseline until about $1 \frac{1 / 2}{2}$ hours after smoking $\left(F_{1,22}=34.67,20.03 \&\right.$ $16.97 ;$ all $p<.001 ; p_{c}=.01, .013 \&$ \& .017). Separate sequential comparisons of subjective feelings of contentedness provided the same results $\left(F_{1,22}=27.24,19.98\right.$ $\&$ 9.99; $\left.p<.001, .001 \& .004 ; p_{c}=.01, .013 \& .017\right)$. Feelings of calmness followed a different profile over time. Separate sequential comparisons showed that no 
significant change occurred during the first few hours after smoking, but at the end of the session subjects felt calmer than they had at baseline $\left(F_{1,22}=12.25\right.$; $\left.p<.002 ; p_{c}=.01\right)$.

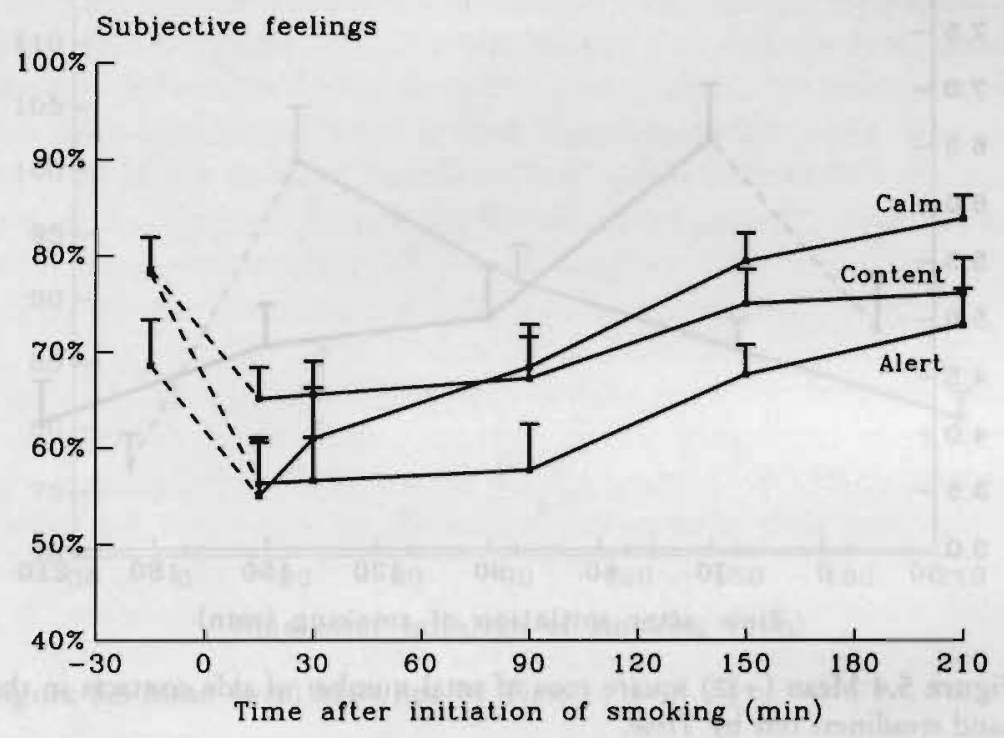

Figure 5.3 Mean (+SE) perceived alertness, contentedness and calmness by Time.

\section{Critical Tracking Test}

The average frequency at which the subjects lost control $\left(\lambda_{i}\right)$ was $4.40 \mathrm{rad} / \mathrm{s}$ before smoking and fell to $4.15 \mathrm{rad} / \mathrm{s}$ in the first test after smoking and gradually rose to baseline level in later tests. MANOVA however failed to show a significant Time effect.

\section{Hand Steadiness Test}

Figure 5.4 demonstrates that subjects' hand steadiness diminished after smoking marijuana, but this effect dissipated rapidly after the first test. On the average, the subjects' hand steadiness was even superior at a time two hours after smoking to what it had been initially.

MANOVA revealed a significant Time effect $\left(\mathrm{F}_{4,18}=6.38 ; p<.002\right)$. Yet separate comparisons showed that hand steadiness was only significantly different from baseline in the first test after smoking $\left(\mathrm{F}_{1,22}=16.89 ; p<.001 ; p_{c}=.013\right)$. 


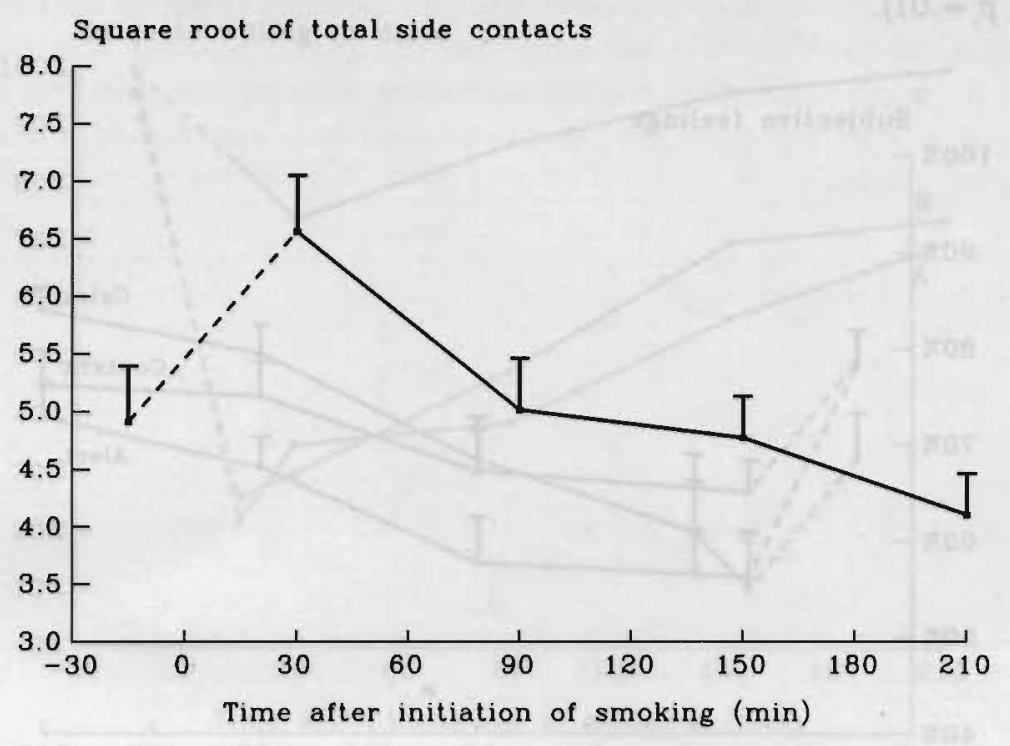

Figure 5.4 Mean (+SE) square root of total number of side contacts in the hand steadiness test by Time.

\section{Heart Rate}

The subjects' average heart rate, presented in Figure 5.5, varied between 75 and 100 beats per minute (bpm) in close accordance with their subjective feelings. Thirty minutes after smoking, the increase in heart rate varied from 2 to $77 \mathrm{bpm}$, with a mean elevation of $24 \mathrm{bpm}$. Heart rate diminished over successive measurements approaching baseline values after $3 \% / 4$ hours. MANOVA revealed a significant Time effect $\left(\mathrm{F}_{4,18}=10.43 ; p<.001\right)$ and separate ANOVA comparisons showed that these elevations were significantly different from baseline at each but the last measurement $\left(\mathrm{F}_{1,22}=35.64,21.90,12.67 \& 2.37 ; p<.001, .001, .002 \& .14 ; p_{c}=.013\right.$, $.017, .025 \& .05$, respectively).

\section{Intra-Subject Relations between Variables}

Table 5.5 shows the average intra-subject correlations between [THC], $\log _{10}$ [THC], and [THC-COOH] with each of the other variables. The log transformation was applied to achieve a more linear and homeoscadastic relationship between THC values and the other variables. The constant of 1 was added to the raw [THC] values for avoiding negative log transformations. It can be concluded from this table that, within subjects, higher plasma levels of the drug were associated with, and probably produced, increased heart rate, increased feelings of perceived 


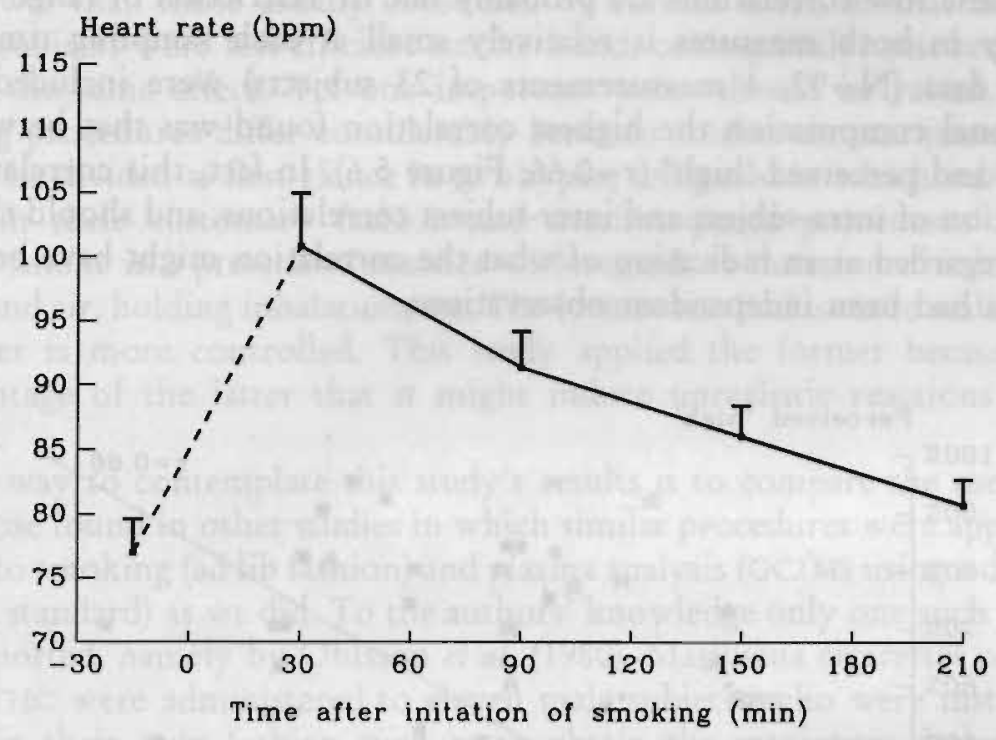

Figure 5.5 Mean (+SE) heart rate by Time.

'high', diminished hand steadiness, and diminished feelings of calmness, contentedness and alertness. The correlation with critical tracking performance was not significant, confirming the insensitivity of the test. The log transformation of [THC] did not change the size of the correlations dramatically.

Table 5.5 Average intra-subject correlations (* $p<.05 *{ }^{* * *} p<.01 ; 2$-tailed).

\begin{tabular}{llll}
\hline & {$[\mathrm{THC}]$} & $\log _{10}([\mathrm{THC}]+1)$ & {$[\mathrm{THC}-\mathrm{COOH}]$} \\
\hline heart rate & $+.79^{* *}$ & $+.82^{* *}$ & $+.68^{* *}$ \\
perceived 'high' & $+.88^{* *}$ & $+.95^{* *}$ & $+.87^{* *}$ \\
$\lambda_{c}$ & -.20 & -.22 & -.27 \\
no. of side contacts & $+.48^{\circ}$ & $+.45^{\circ}$ & +.39 \\
calmness & $-.51^{\circ}$ & -.38 & +.02 \\
contentedness & $-.57^{* *}$ & $-.59^{* *}$ & $-.46^{*}$ \\
alertness & $-.64^{* *}$ & $-.72^{* *}$ & $-.66^{* *}$ \\
\hline
\end{tabular}

\section{Inter-Subject Relations between Variables}

Inter-subject correlations between plasma concentrations of the drug and measures of subjective feelings, heart rate and performance were typically low $(r<.50)$ at each sampling time. The correlations between $\log [\mathrm{THC}]+1$ and 
perceived 'high', for example, were $r=0.07$ (ns), 0.19 (ns), $0.43(p<.05)$ and 0.35 (ns), at $t=40,100,160$ and 220 minutes after initiation of smoking, respectively. These low correlations are probably due to 'restriction of range', i.e. the variability in both measures is relatively small at each sampling time. If all available data ( $N=92,4$ measurements of 23 subjects) were included in the correlational computation the highest correlation found was that between log [THC]+1 and perceived 'high' ( $r=0.66$; Figure 5.6). In fact, this correlation is a combination of intra-subject and inter-subject correlations, and should therefore only be regarded as an indication of what the correlation might have been if all these data had been independent observations.

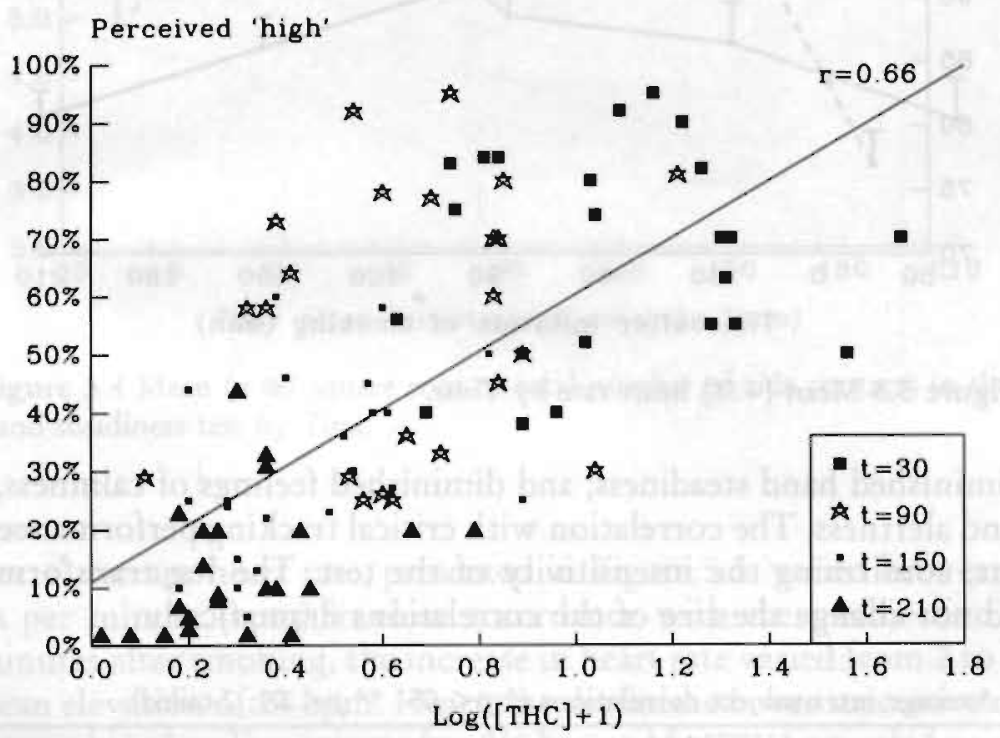

Figure 5.6 Scatter diagram of $\log ([\mathrm{THC}]+1)$ in plasma and perceived 'high'.

Regression line comprising all data is inserted.

\subsection{Discussion}

The laboratory study's major purpose was to determine the amount of THC recreational users of cannabis smoke to reach a comfortable 'high' and to establish from these results the maximum dose for subsequent driving studies. Median and mean amount of THC consumed were 308 and $292 \mu \mathrm{g} / \mathrm{kg}$ respectively. From these results it was decided that the maximum dose for subsequent driving studies would be $300 \mu \mathrm{g} / \mathrm{kg}$. This is considerably higher than doses that 
have usually been administered to subjects in experimental studies (typical, $100-200 \mu \mathrm{g} / \mathrm{kg}$ THC). This could mean either that previous studies' THC doses were lower than those usually consumed by current users, or that the present study's subjects were less efficient smokers and, consequently, smoked more to achieve the same effect. Yet one important point should be raised. Imposed smoking procedures differ considerably between studies on marijuana's effects and can be divided at first glance in two types, self-paced in which subjects may smoke in their customary fashion and machine-paced procedures in which subjects smoke in a prescribed manner with regards to duration of inhalation of smoke and air, holding inhalation, etc. The former method is more realistic while the latter is more controlled. This study applied the former because of the disadvantage of the latter that it might induce unrealistic reactions in some subjects.

One way to contemplate this study's results is to compare the mean [THC] with those found in other studies in which similar procedures were applied with respect to smoking (ad lib fashion) and plasma analysis (GC/MS using a deuterated internal standard) as we did. To the authors' knowledge only one such study has been reported, namely by Ohlsson et al. (1980). Marijuana cigarettes containing $19 \mathrm{mg}$ THC were administered to eleven male subjects who were instructed to smoke in their own fashion such as to obtain the maximum desired 'high'. Subsequent gravimetric estimation showed that a mean of $13.0 \mathrm{mg}$ THC was consumed. [THC] values ranged between 5.4 and 18.0 (mean 12.4) $\mathrm{ng} / \mathrm{ml} 30$ minutes after termination of smoking. Only the males of the present study should be included in the comparison with Ohlsson's study, because of the significant difference found between [THC] values of both sexes in the current study. Eleven males in the present study smoked $22.3 \mathrm{mg}$ THC, on average, and their plasma concentrations ranged between 6.7 and 45.9 (mean 17.7 ) $\mathrm{ng} / \mathrm{ml} 40$ minutes after initiation of smoking. Thus, our subjects smoked $70 \%$ more THC than Ohlsson's subjects did, resulting in a $43 \%$ higher THC level in plasma, as measured 30 minutes after smoking. The average [THC] found in the present study was therefore in the expected direction. This observation may lead to the tentative conclusion that the preferred THC dose to reach a desired 'high' in the present study was not due to inefficient smoking but to the fact that current marijuana (or hashish) users do smoke higher THC doses than previously administered in scientific studies.

Other results from the present study showed that perceived 'high' and heart rate are very sensitive (psychological and physiological) measures of marijuana intoxication which confirms prior research. Impairments in laboratory tests performance were measured at the time of peak subjective feelings but generally, objective impairment dissipated more rapidly than the feelings themselves. All objective signs of impaired functions were gone within 1.5 hours after smoking. The explanation may be that practice and habituation effects, or both, occurred 
during the session concealing marijuana's impairment, or, that procedural errors have been made in administering these tests. No definitive answer can be provided and no conclusions can be drawn from this study with respect to marijuana's effects upon performance because of the lack of a control group. The study was simply not designed to estimate these effects, only to indicate whether either of these measures should be considered for inclusion in later studies. If any measure appeared to be systematically related to the inferred changes in THC's pharmacological activity over time, this measure might assume a high degree of practical importance. Correlational analyses, however, showed no strong relationships between $[\mathrm{THC}]$ or $[\mathrm{THC}-\mathrm{COOH}]$ and performance in any test.

An important issue relating to traffic safety is whether subjects would drive a car while under the influence of marijuana. Although all subjects had admitted driving a car while intoxicated at least once before, a majority (about $65 \%$ ) of the subjects was not willing to drive a car for relatively unimportant reasons shortly after smoking when experiencing the drug's peak subjective reaction. However, most said they would drive, for a very urgent reason. On one hand, this means that the majority of the subjects are aware of a potential marijuana related driving impairment; on the other, a sizable minority (35\%) would not refrain from driving a car for unimportant reasons when they are experiencing a 'high'. These subjects in particular are a source of concern with respect to traffic safety, if marijuana smoking indeed impairs driving performance. Two questions that arise from these contemplations were addressed by the succeeding driving studies; namely, 1 . does marijuana adversely affect driving performance, and 2 . is willingness to drive after marijuana smoking related to driving impairment? 


\section{CHAPTER 6}

\section{MARIJUANA AND DRIVING ON A RESTRICTED HIGHWAY}

\subsection{Introduction}

As mentioned in Chapter 3, THC's effects on actual driving performance have been assessed in a relatively small number of studies and only once in the presence of other traffic. In those studies, the effects of doses up to about $250 \mu \mathrm{g} / \mathrm{kg}$ were modest, if present at all. These findings provided some assurance that it would be safe for subjects to undertake carefully supervised driving tests on normal roads and in traffic, even after treatment with the somewhat higher average dose (i.e. $300 \mu \mathrm{g} / \mathrm{kg}$ ) that was preferred by regular marijuana users in the laboratory study. Nonetheless normal prudence demanded a demonstration of the test's safety in an environment resembling reality but where neither the subjects nor other road users would be endangered if the optimistic forecast proved false. One objective of the present study was to provide that demonstration using a standard test on a highway closed to other traffic.

The second objective was to define the dose-effect relationship between inhaled THC dose and that parameter of vehicular control which is measured in the standard test; i.e. standard deviation of lateral position (SDLP), an index of 'weaving' amplitude the subject allows while attempting to maintain a constant speed and steady lateral position between traffic lane boundaries during uninterrupted highway driving. Alcohol's effects on SDLP were previously measured by Louwerens et al. $(1985,1987)$ in practically the same manner as THC's in this study. The earlier results showing a nearly perfect $(r=0.99)$ exponential relationship between mean blood alcohol concentration (0-0.12 g\%) and mean SDLP for 24 'social drinkers', serve admirably for evaluating THC's effects in the present case.

Other objectives were to measure changes in SDLP from tests after placebo to those following separate THC doses of 100,200 and $300 \mu \mathrm{g} / \mathrm{kg}$; and, to relate these objective measures of driving impairment to subjective impressions of driving quality and expressed willingness to drive in the same states of intoxication under normal circumstances. 


\subsection{Methods}

\section{Subjects}

The same twelve men and twelve women who participated in the laboratory study served again as the subjects. Plasma from the laboratory study was still not analyzed at the time data collection commenced. The male that apparently did not inhale marijuana smoke was therefore not dropped from the study. As before, his plasma samples showed neither THC nor THC-COOH. Data from this subject had to be again excluded from further analyses. Characteristics of the remaining 23 subjects are shown in the previous chapter (Table 5.1).

\section{Design, Doses and Administration}

Subjects were treated on separate occasions with THC doses of 0 (placebo), 100, 200 , and $300 \mu \mathrm{g} / \mathrm{kg}$. Marijuana cigarettes were prepared from batches containing $1.75 \%$ THC for the two lowest, and $2.57 \%$ THC for the highest dose. Cigarettes were cut to different lengths to provide the doses appropriate for the individuals' body weights. It was necessary to provide the five largest subjects with two cigarettes at a time since one would not contain the total dose. Subjects smoked the cigarettes through a plastic holder, ad lib, but they were encouraged to smoke the entire dose in ten minutes. After cessation of smoking, cigarettes were retained for subsequent gravimetric estimation of THC consumed. These analyses revealed that the average $( \pm \mathrm{SD})$ amount of consumed THC in the three marijuana conditions was $6.8( \pm 0.9), 13.6( \pm 1.9)$ and $20.4( \pm 2.8) \mathrm{mg}$, which equals $94( \pm 4)$, $186( \pm 13)$ and $282( \pm 18) \mu \mathrm{g} / \mathrm{kg}$, respectively, or about $6 \%$ less than target doses. Order of treatments were counterbalanced. They were administered subject- and observer-blind (i.e. the investigator who prepared the treatments was not involved in their administration or with data collection).

\section{Testing Procedures}

Eight subjects were tested per night and all 24 within a week. Subjects were tested at the same times and on the same days of the week for four consecutive weeks. Breath and urine tests were executed upon the subjects' arrival to check for the presence of alcohol and illicit drugs. If cannabinoids were found in the urine, a blood sample was taken for later verification of the presence of THC.

Two subjects commenced smoking at a time at $t=0$ (Table 6.1). Driving tests were performed twice, beginning at $t=40$ and 100 minutes and lasting 15-20 minutes. Blood samples were taken before the driving tests. The subjects' pulse was taken and their performance measured in two laboratory tests that began after the driving tests. Subjective assessments were made immediately after smoking, and before and after the driving tests. Before the start of the experiment, subjects were individually trained to operate the vehicle under generally the same conditions as the tests later occurred. 
Table 6.1 Schedule of activities on test-days.

\begin{tabular}{ll}
\hline relative time $(\mathrm{min})$ & activity \\
\hline $0-10$ & smoking \\
$30-35$ & blood sampling \\
$40-60$ & standard driving test \\
$70-80$ & tracking and hand steadiness tests \\
& heart rate and blood Pressure \\
$90-95$ & blood sampling \\
$100-120$ & standard driving test \\
$130-140$ & tracking and hand steadiness tests \\
& heart rate and blood pressure \\
\hline
\end{tabular}

\section{Driving Test}

The driving test, developed and standardized by O'Hanlon et al. $(1982,1986)$ and applied in more than 40 open- and closed-road studies by three Dutch Institutes during the last decade, measures the ability to control an instrumented vehicle's speed and lateral position. Subjects were instructed to maintain speed at $90 \mathrm{~km} / \mathrm{h}$ (56 mph), or less if they felt incapable of driving safely at that speed, and a steady lateral position between the delineated boundaries of the traffic lane.

Driving was performed over a $11 \mathrm{~km}(6.8 \mathrm{mi})$ section of a primary highway (A76) that connects the Dutch cities of Geleen and Heerlen. Two lanes in the same direction were closed to normal traffic between the hours of 19.00 and 24.00 on three consecutive week-nights over four consecutive weeks of testing. Driving began at one end of the section, involved turning at the other and ended with a return to the origin. A licensed driving instructor accompanied each subject. He was charged with responsibility for ensuring safety at all times and was able to intervene, if necessary, using redundant vehicular controls.

Two Volvo station wagons containing essentially the same instrumentation were employed in the study. The first of a pair of subjects who received treatments together departed from the origin driving one vehicle and was followed by the second driving the other after $2 \frac{1}{2} 2$ minutes. The first subject waited for the arrival of the second at the turning point before returning to the origin. The purpose was to avoid having the subjects, travelling in opposite directions, meet en route. The major instrumentation comprised devices for acquiring continuous analog signals representing steering wheel angle, vehicle speed and lateral position relative to the midline stripe delineation, and a computer system for recording those signals continuously at a $4 \mathrm{~Hz}$ sampling rate.

The primary dependent variable was the standard deviation of lateral position (SDLP), which has been shown to be both highly reliable (typical test-retest correlation of $0.7-0.9)$ and very sensitive to the influence of sedative drugs and 
alcohol. Other dependent variables were mean speed (SP) and standard deviation of speed (SDSP) and steering wheel angle (SDST).

\section{Questionnaires}

The same subjective questionnaires used in the laboratory study were administered to the subjects immediately after cessation of smoking $(t=10)$ and again at the beginning of each driving test $(t=40 \& 100)$. At the end of each driving test $(t=60 \& 120)$, the subjects were required to retrospectively rate their effort made while performing the test (Zijlstra and Van Doorn, 1985; Meyman and Zijlstra, 1986) and subjective driving quality on respective visual-analog scales. Scores on these scales will be expressed as percentage of total scale and percentage of 'normal' driving quality, respectively.

\section{Laboratory Tests}

Two of the tests employed in the laboratory study were also applied here, namely the critical tracking and hand steadiness tests. Exactly the same procedures were employed in their administration as described in the previous chapter.

\section{Physiological Assessments}

Heart rate, systolic and diastolic blood pressure were measured by means of a digital blood pressure monitor prior to the hand steadiness test.

\section{Blood Sampling}

Blood samples were taken by venepuncture. The samples ( 2 aliquots containing $10 \mathrm{ml}$ each) were heparinized and centrifuged. The plasma fractions were placed in frozen $\left(-20^{\circ} \mathrm{C}\right)$ storage prior to analysis for [THC] and [THC-COOH].

\section{Data Analysis}

All data measured on ratio or interval scales were taken in a mixed betweengroups, within-subjects MANOVA analysis. Sex was the between-groups factor. Dose (4 levels) and Time after dosing ( 2 or 3 levels) were factors tested withinsubjects. If a significant $(p<.05)$ Dose effect was found, repeated measures ANOVAs were conducted for testing differences between measures obtained after placebo and each THC dose, separately. Data in these cases were collapsed across Sex and Time unless the MANOVA analysis had revealed a significant interaction between either factor and Dose. In the figures, the mean of the variable is depicted by the height of the bar and its standard error (SE) by the height of the vertical line above the bar.

Separate dose effects were tested using the 'Sequential Bonferroni' procedure for adjusting the $\alpha$-probability criterion $\left(p_{c}\right)$ in accordance with the number of separate comparisons in a given set (Overall and Rhoades, 1987). This means that for the largest of three differences tested at once, $p$ had to be less than $.05 / 3=$ 
.017 to be judged significant. For the second, it had to be $<.05 / 2=.025$; and for the smallest difference, $p<.05$. The adjustment had the effect of holding the probability of making a type-I error at $p \leq .05$ over the entire set of comparisons.

The subjects' expressions of willingness to drive were made on the basis of a dichotomous decision and could not for that reason be analyzed in the same manner as other variables. These data were therefore analyzed using Cochran's Q-statistic test for assessing differences between dosing conditions at each time of testing, separately.

Significant Sex effects were generally absent. Results pertaining to differences between the sexes are therefore only reported for the exceptional cases where the differences were significant.

Inter-subject and intra-subject correlations were computed and tested as described in the previous chapter.

\subsection{Results}

\section{Plasma Concentrations of the Drug}

Though consumed dose differed little between subjects, [THC] and [THC-COOH] varied enormously. Thirty minutes after smoking $300 \mu \mathrm{g} / \mathrm{kg}$, for example, [THC] ranged between 1.6 and $59.6 \mathrm{ng} / \mathrm{ml}$. Table 6.2 shows mean, median and range of [THC] and [THC-COOH] by Dose and Time. Placebo values were not used in the statistical analyses since these were zero in most cases.

Table 6.2 Mean, median and range of [THC] and [THC-COOH] in $\mathrm{ng} / \mathrm{ml}(\mathrm{N}=23)$.

\begin{tabular}{|c|c|c|c|c|c|c|c|}
\hline & & \multicolumn{2}{|c|}{$100 \mu \mathrm{g} / \mathrm{kg}$} & \multicolumn{2}{|c|}{$200 \mu \mathrm{g} / \mathrm{kg}$} & \multicolumn{2}{|c|}{$300 \mu \mathrm{g} / \mathrm{kg}$} \\
\hline & & $t=30$ & $t=90$ & $t=30$ & $t=90$ & $t=30$ & $t=90$ \\
\hline \multirow{3}{*}{ [THC] } & mean & 9.5 & 3.5 & 15.9 & 4.8 & 20.7 & 6.2 \\
\hline & median & 9.0 & 3.2 & 12.0 & 4.3 & 19.1 & 5.6 \\
\hline & range & $0.0-21.3$ & $0.0-11.0$ & $1.7-39.3$ & $0.0-11.8$ & $1.6-59.6$ & $0.8-15.4$ \\
\hline \multirow{3}{*}{ [THC-COOH] } & mean & 10.9 & 9.4 & 14.2 & 12.1 & 17.5 & 15.2 \\
\hline & median & 7.2 & 4.7 & 13.4 & 9.6 & 13.8 & 11.4 \\
\hline & range & $0.0-61.9$ & $0.0-55.5$ & $2.2-73.9$ & $2.0-65.2$ & $2.6-64.4$ & $2.6-55.4$ \\
\hline
\end{tabular}

As shown by Table 6.2 plasma concentrations of the drug were clearly related to the administered dose and time of blood sampling. MANOVA confirmed this observation yielding a significant Dose $\left(\mathrm{F}_{2,20}=14.65 \& 16.59\right.$ for [THC] and [THC-COOH], respectively; both $p<.001)$ and Time $\left(\mathrm{F}_{1,21}=50.76 \& 21.16\right.$; both $p<.001)$ effect. There was a significant Dose by Time interaction for [THC] 
$\left(\mathrm{F}_{2,20}=10.07 ; p<.001\right)$ and not $[\mathrm{THC}-\mathrm{COOH}]$. Though not shown in the table, males had significantly higher [THC-COOH] values, $9 \mathrm{ng} / \mathrm{ml}$ on average, than females $\left(F_{1,21}=4.49 ; p<.05\right)$; average $[\mathrm{THC}]$ values were virtually the same for both sexes.

Perceived 'bigh'

Average subjective feelings of intoxication ('high') were dose-related and highest just before the first driving test (Figure 6.1). Relative to maximum personal experience, peak levels of intoxication were about $30 \%, 50 \%$ and $60 \%$ after 100 , 200 and $300 \mu \mathrm{g} / \mathrm{kg}$ doses, respectively. Feelings of 'high' after placebo were near zero in most cases. Therefore changes occurring after marijuana smoking, relative to placebo, instead of the raw values, were analyzed by MANOVA. Change scores were significantly different from zero $\left(F_{1,21}=125.22 ; p<.001\right)$, dose-related $\left(F_{2,20}=21.76 ; p<.001\right)$ and time-related $\left(F_{2,20}=36.68 ; p<.001\right)$. Females felt more intoxicated than males $\left(F_{1,21}=4.59 ; p<.05\right)$, but there was no significant Sex by Dose interaction. Univariate analyses revealed that intoxication ratings were different from zero and followed a quadratic trend in all marijuana conditions.

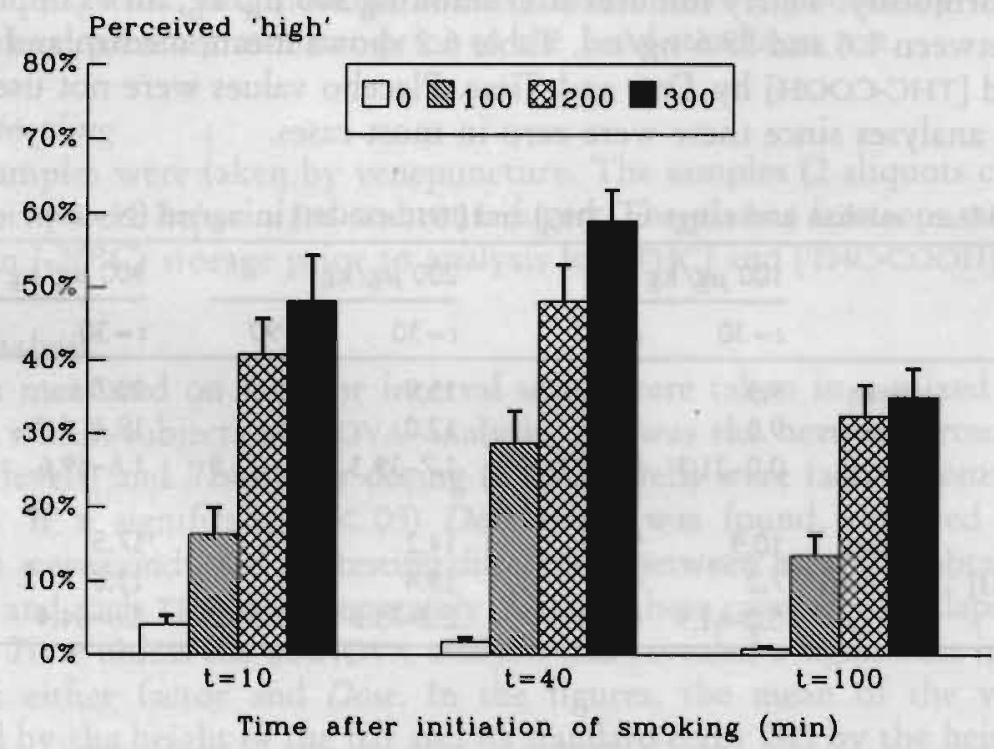

Figure 6.1 Mean (+SE) perceived 'high' by Dose and Time. 


\section{Driving Performance}

No driving tests had to be stopped for safety reasons by the driving instructors. Yet in two instances, both after the highest THC dose, the instructor felt compelled to intervene. In one case, the driving instructor twice warned the subject to avoid a screwdriver laying on the road but when he failed to react the instructor did by steering away from the object (it was immediately removed following this incident). The subject was queried about this situation after termination of the ride. He recalled that the driving instructor had taken control but did not recall why! In the other case, a subject failed to decelerate as he approached the turning point. The instructor told him to do so whereupon the subject abruptly brought the vehicle to a stop using the break.

SDLP reliability was high in this study: the correlation between measurements made on successive trials after placebo was $0.92(p<.001)$. Correlations between SDLP values on the first and second trials after THC doses of 100,200 and $300 \mu \mathrm{g} / \mathrm{kg}$ were $0.90,0.91$ and 0.91 (all $p<.001$ ), respectively. This means that subjects' SDLPs from the second ride were almost perfectly related to those of the first, and, consequently, that SDLP measurement error was very small.

Mean values of SDLP are shown in Figure 6.2 as a function of Dose and Time. Higher values, indicating poorer road tracking performance, were found after marijuana smoking. MANOVA revealed that the Sex effect $\left(F_{1,21}=4.41 ; p<.05\right)$ was

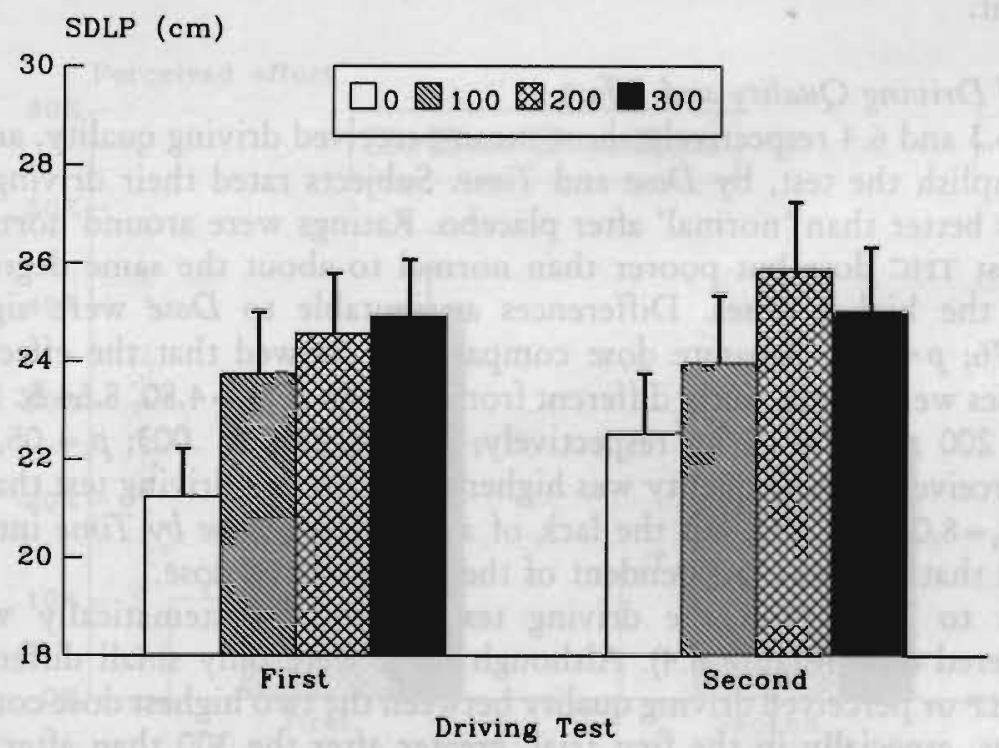

Figure 6.2 Mean (+SE) SDLP by Dose and Time. 
significant, females having higher SDLPs than males. The Sex by Dose interaction, however, was not significant $\left(\mathrm{F}_{3,19}=2.54 ; p<.09\right)$, indicating that the effect of marijuana was grossly the same for both sexes. The analysis indicated a clear Dose effect $\left(\mathrm{F}_{3,19}=8.92 ; p<.001\right)$. Neither Time nor Dose by Time effects were statistically significant indicating that impairment after marijuana was the same in both trials. Separate dose comparisons revealed that performance after marijuana was always worse than after placebo $\left(F_{1,22}=7.45,19.96 \& 14.58\right.$ for the $100,200 \&$ $300 \mu \mathrm{g} / \mathrm{kg}$ conditions, respectively; $p<.012, .001 \& .001 ; p_{c}=.05, .017 \& .025$ ).

Mean speed was very close to that established as the target by instructions and varied between conditions from 88.9 to $90.5 \mathrm{~km} / \mathrm{h}(55.3$ to $56.2 \mathrm{mph})$. Subjects drove $0.4 \mathrm{~km} / \mathrm{h}(0.25 \mathrm{mph})$ faster after the $100 \mu \mathrm{g} / \mathrm{kg}$ dose, and $0.4 \mathrm{~km} / \mathrm{h}$ slower after both of the higher doses, than after placebo. Yet these differences were small as percentages of the average, and not significant. On average, speed was $0.6 \mathrm{~km} / \mathrm{h}(0.37 \mathrm{mph})$ higher in the second than in the first ride resulting in a significant Time effect $\left(\mathrm{F}_{1,21}=5.24 ; p<.04\right)$.

Differences in standard deviation of speed were greater between sexes than between conditions. There was a significant Sex effect $\left(F_{1,21}=6.99 ; p<.02\right)$, females having greater difficulty in maintaining a constant speed than males. SDSP was, after each THC dose, higher in the first, but lower in the second ride relative to placebo. Changes from placebo were, however, small and not significant.

Changes in standard deviations of steering wheel angle were small and not significant.

\section{Perceived Driving Quality and Effort}

Figures 6.3 and 6.4 respectively show mean perceived driving quality, and effort to accomplish the test, by Dose and Time. Subjects rated their driving performance as better than 'normal' after placebo. Ratings were around normal after the lowest THC dose but poorer than normal to about the same degrees after both of the higher doses. Differences attributable to Dose were significant $\left(F_{3,19}=3.76 ; p<.03\right)$. Separate dose comparisons showed that the effects of all three doses were significantly different from placebo $\left(\mathrm{F}_{1,22}=4.80,8.64 \& 10.76\right.$ for the 100,200 and $300 \mu \mathrm{g} / \mathrm{kg}$ respectively; $p<.04, .008 \& .003 ; p_{c}=.05, .025 \&$ $.017)$. Perceived driving quality was higher in the second driving test than in the first $\left(\mathrm{F}_{1,21}=8.06 ; p<.01\right)$, but the lack of a significant Dose by Time interaction indicated that this was independent of the administered dose.

Effort to accomplish the driving test increased systematically with the administered dose (Figure 6.4). Although there were only small differences in either SDLP or perceived driving quality between the two highest dose conditions, effort was, especially in the first trial, greater after the 300 than after the 200 $\mu \mathrm{g} / \mathrm{kg}$ dose. This suggests that subjects had to compensate more after the highest dose to achieve about the same objective and subjective driving performance. 


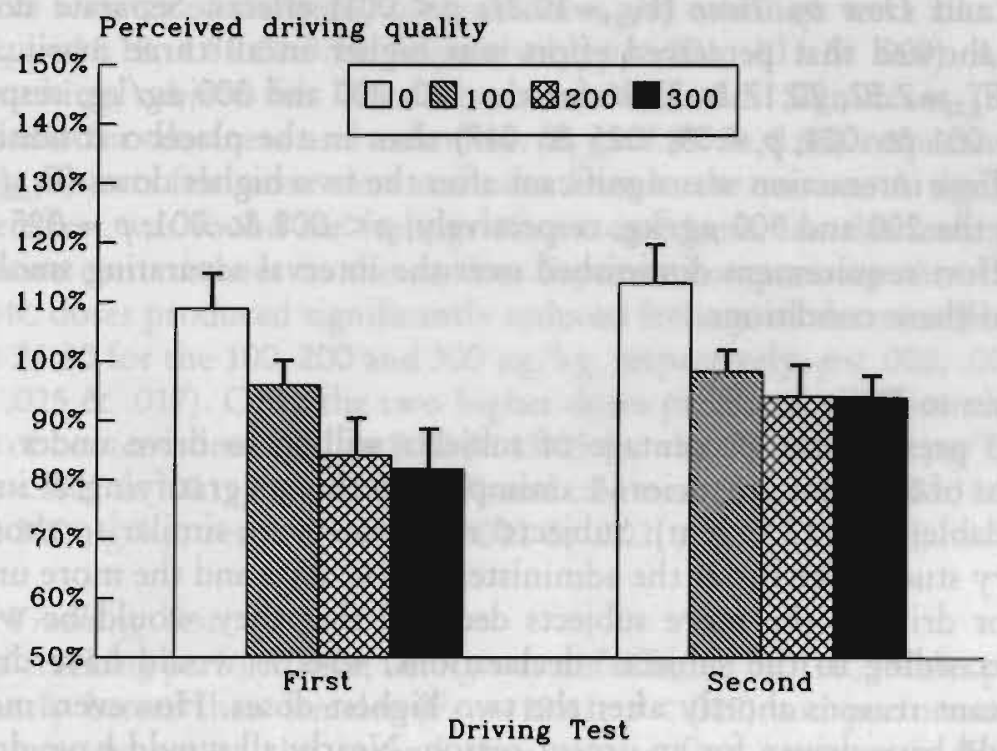

Figure 6.3 Mean (+SE) perceived driving quality, expressed as percentage of 'normal', by Dose and Time.

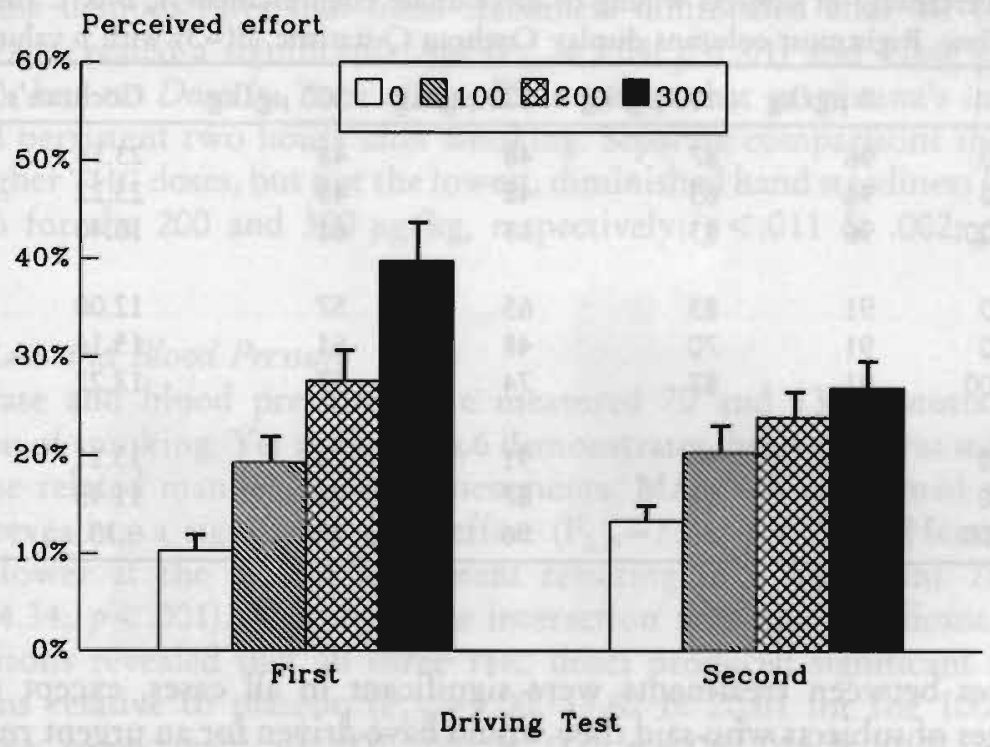

Figure 6.4 Mean (+SE) perceived effort to accomplish the test, expressed as percentage of maximum of scale, by Dose and Time. 
MANOVA revealed significant Dose $\left(\mathrm{F}_{3,19}=13.41 ; p<.001\right)$, Time $\left(\mathrm{F}_{1,21}=8.59\right.$; $p<.008)$ and Dose by Time $\left(\mathrm{F}_{3,19}=10.27 ; p<.001\right)$ effects. Separate dose comparisons showed that perceived effort was higher in all three marijuana conditions $\left(F_{1,22}=7.57,20.17 \& 37.96\right.$ for the 100,200 and $300 \mu \mathrm{g} / \mathrm{kg}$, respectively; $\left.p<.012, .001 \& .001 ; p_{c}=.05, .025 \& .017\right)$ than in the placebo condition. The Dose by Time interaction was significant after the two higher doses $\left(F_{1,22}=8.45 \&\right.$ 24.95 for the 200 and $300 \mu \mathrm{g} / \mathrm{kg}$, respectively; $p<.008 \& 2.001 ; p_{c}=.025 \& .017$ ); i.e. the effort requirement diminished over the interval separating smoking and driving in these conditions.

\section{Willingness to Drive}

Table 6.3 presents the percentage of subjects willing to drive under specified conditions of different urgencies ( $A$. unimportant though gratifying; $B$. important but avoidable; and, $C$. urgent). Subjects' responses were similar to those in the laboratory study. The lower the administered THC dose and the more urgent the reason for driving, the more subjects declared that they would be willing to drive. According to the subjects' declarations, $40-60 \%$ would have driven for unimportant reasons shortly after the two highest doses. However, more than $75 \%$ would have driven for an urgent reason. Nearly all would have driven $11 / 2$ hours after smoking for an urgent reason when objectively measured driving performance was still impaired.

Table 6.3 Percentage of subjects willing to drive under circumstances $A, B \& C$ (see text) by Dose and Time. Rightmost columns display Cochran Q-statistic $(\mathrm{df}=3)$ with $p$ values.

\begin{tabular}{|c|c|c|c|c|c|c|c|}
\hline \multicolumn{2}{|c|}{ 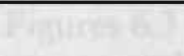 } & $0 \mu \mathrm{g} / \mathrm{kg}$ & $100 \mu \mathrm{g} / \mathrm{kg}$ & $200 \mu \mathrm{g} / \mathrm{kg}$ & $300 \mu \mathrm{g} / \mathrm{kg}$ & Cochran's Q & $p<$ \\
\hline \multirow{3}{*}{ A } & $t=10$ & 96 & 87 & 48 & 48 & 23.54 & .001 \\
\hline & $t=40$ & 96 & 65 & 48 & 43 & 23.22 & .001 \\
\hline & $t=100$ & 96 & 83 & 65 & 61 & 16.40 & .001 \\
\hline \multirow{3}{*}{ B } & $t=10$ & 91 & 83 & 65 & 57 & 12.00 & .008 \\
\hline & $t=40$ & 91 & 70 & 48 & 61 & 15.14 & .002 \\
\hline & $t=100$ & 91 & 87 & 74 & 57 & 17.22 & .001 \\
\hline \multirow{3}{*}{ C } & $t=10$ & 100 & 96 & 91 & 74 & 13.11 & .005 \\
\hline & $t=40$ & 100 & 96 & 87 & 74 & 11.45 & .01 \\
\hline & $t=100$ & 100 & 96 & 96 & 87 & 6.33 & ns \\
\hline
\end{tabular}

Differences between treatments were significant in all cases, except one: the percentages of subjects who said they would have driven for an urgent reason 100 minutes after initiation of smoking were not different between treatment conditions. 


\section{Perceived Alertness, Contentedness and Calmness}

Subjective feelings of alertness, contentedness and calmness were all affected by Dose $\left(\mathrm{F}_{3,19}=11.18,4.86 \& 5.14\right.$, respectively; $\left.p<.001, .011 \& .009\right)$. After marijuana smoking, subjects felt less alert, content and calm. Significant Time effects were found for alertness and calmness $\left(F_{2,20}=7.89 \& 8.10\right.$, respectively; both $p<.003$ ): subjects felt more alert and calm later in the session. A significant Sex by Time effect was found for feelings of contentedness, females feeling less and males more content later in the session. Separate comparisons showed that all three THC doses produced significantly reduced feelings of alertness $\left(F_{1,22}=12.46\right.$, $28.94 \& 24.80$ for the 100,200 and $300 \mu \mathrm{g} / \mathrm{kg}$, respectively; $p<.002, .001 \& .001$; $\left.p_{c}=.05, .025 \& .017\right)$. Only the two higher doses produced significantly reduced feelings of contentedness $\left(F_{1,22}=8.70 \& 15.05\right.$ for the 200 and $300 \mu \mathrm{g} / \mathrm{kg}$, respectively; $\left.p<.007 \& .001 ; p_{c}=.025 \& .017\right)$ and calmness $\left(\mathrm{F}_{1,22}=14.29 \& 11.70\right.$ for the 200 and $300 \mu \mathrm{g} / \mathrm{kg}$, respectively; $\left.p<.001 \& .002 ; p_{\mathrm{c}}=.017 \& .025\right)$.

\section{Critical Tracking Test}

Subjects' tracking performance, i.e. $\lambda_{c}$, was not affected by THC. Males performed significantly better than females $\left(\mathrm{F}_{1,21}=12.61 ; p<.002\right)$ and performance of all subjects was worse at the second than at the first assessment $\left(F_{1,21}=10.89\right.$; $p<.003$ ), but these observations are not of great concern.

\section{Hand Steadiness Test}

Figure 6.5 demonstrates that hand steadiness diminished after all THC doses. MANOVA revealed a significant Dose $\left(\mathrm{F}_{3,19}=5.04 ; p<.01\right)$ and Time $\left(\mathrm{F}_{1,21}=8.61\right.$; $p<.008)$, but no Dose by Time effect. This means that marijuana's impairment was still persistent two hours after smoking. Separate comparisons showed that both higher THC doses, but not the lowest, diminished hand steadiness $\left(\mathrm{F}_{1,22}=7.67\right.$ $\& 11.76$ for the 200 and $300 \mu \mathrm{g} / \mathrm{kg}$, respectively; $p<.011 \& .002 ; p_{c}=.025 \&$ $.017)$.

\section{Heart Rate and Blood Pressure}

Heart rate and blood pressure were measured 70 and 130 minutes after the initiation of smoking. Yet as Figure 6.6 demonstrates, heart rate was still elevated in a dose related manner at both assessments. MANOVA confirmed this observation revealing a significant Dose effect $\left(\mathrm{F}_{3,19}=7.71 ; p<.001\right)$. Heart rate was always lower at the second assessment resulting in a significant Time effect $\left(F_{1,21}=24.34 ; p<.001\right)$. Dose by Time interaction was not significant. Separate comparisons revealed that all three THC doses produced significant heart rate elevations relative to placebo $\left(F_{1,22}=9.00,13.62 \& 20.61\right.$ for the 100,200 and $300 \mu \mathrm{g} / \mathrm{kg}$, respectively; $\left.p<.007, .001 \& .001 ; p_{\mathrm{c}}=.05, .025 \& .017\right)$. 


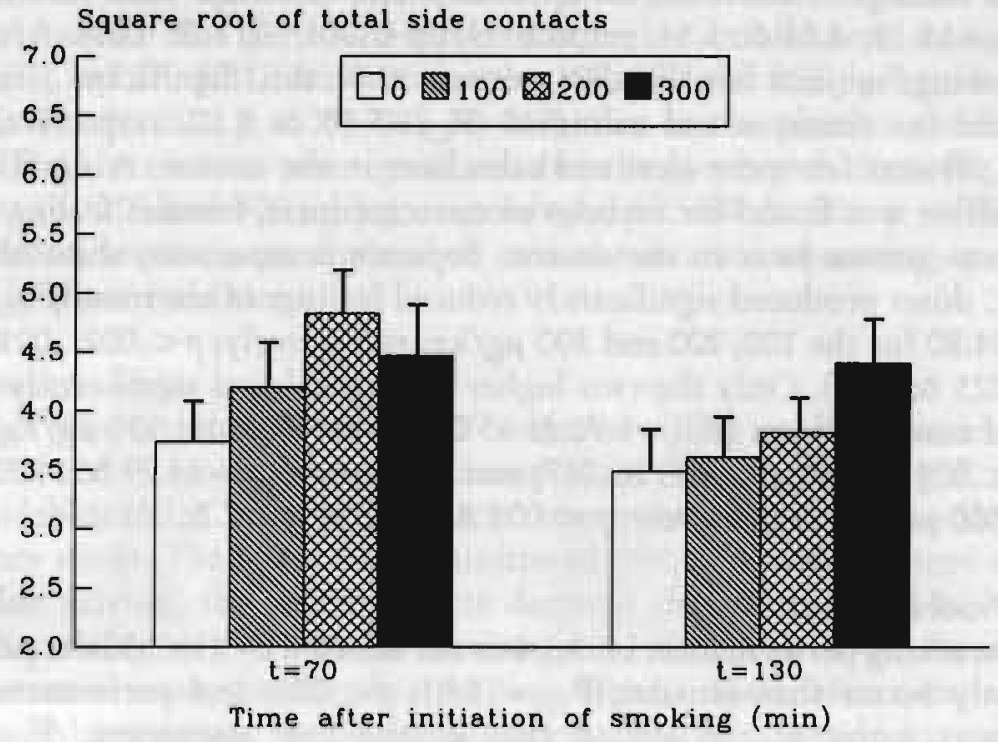

Figure 6.5 Mean (+SE) square root of total number of side contacts in the hand steadiness test by Dose and Time.

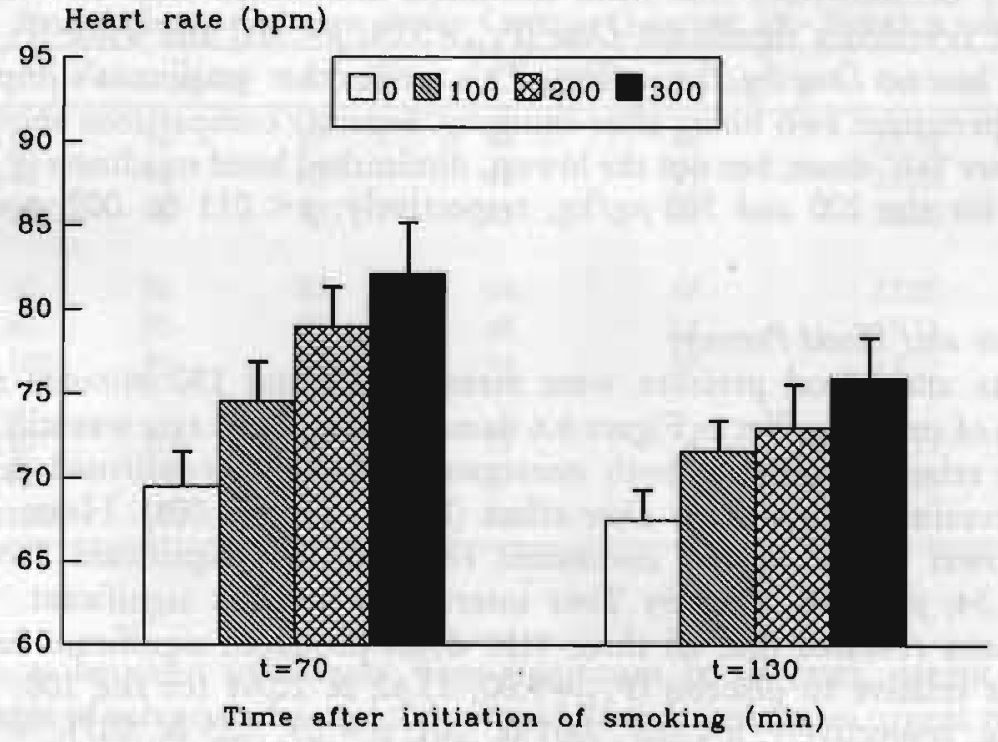

Figure 6.6 Mean (+SE) heart rate by Dose and Time. 
Systolic and diastolic blood pressure measurements were simultaneously analyzed in one 'doubly' repeated measures design. This means that both measures are analyzed in one multivariate design. Univariate effects of THC on systolic and diastolic pressures were only tested for significance, separately, if the multivariate Dose effect was significant. The lowest THC dose produced slightly lower blood pressure relative to placebo, whereas the highest dose had the opposite effect. Changes in blood pressures varied between -2.0 to $+5.8 \mathrm{mmhg}$. MANOVA failed, however, to reveal either a significant Dose or Dose by Time effect. The only significant factor was Time $\left(F_{1,21}=6.92 ; p<.005\right)$, indicating that blood pressure decreased significantly from the first to the second assessment: this occurred in both the systolic $\left(F_{1,21}=12.92 ; p<.002\right)$ and diastolic blood pressure $\left(F_{1,21}=4.77\right.$; $p<.05)$.

\section{Intra-Subject Relations between Variables}

Table 6.4 shows the average intra-subject correlations of [THC], [THC-COOH], and SDLP with each of the other variables. The averages were computed from 23 intra-subject correlations, calculated from data obtained at eight sampling times (twice in each condition).

Table 6.4 Average intra-subject correlations (" $p<.05 * p<.01 ; 2$-tailed).

\begin{tabular}{llll}
\hline & {$[\mathrm{THC}]$} & {$[\mathrm{THC}-\mathrm{COOH}]$} & SDLP \\
\hline perceived 'high' & $+.83^{* *}$ & $+.80^{* *}$ & +.41 \\
perceived effort & $+.53^{* *}$ & $+.58^{* *}$ & +.30 \\
perceived driving quality & $-.43^{*}$ & $-.44^{*}$ & -.29 \\
SDLP & +.23 & +.35 & 1.00 \\
SP & -.35 & -.34 & +.05 \\
SDSP & -.02 & +.05 & +.16 \\
$\lambda_{c}$ & +.11 & +.07 & -.22 \\
no. of side contacts & +.26 & +.20 & +.14 \\
heart rate & $+.49^{*}$ & +.39 & +.27 \\
\hline
\end{tabular}

The table shows that higher plasma levels of the drug were associated with increased feelings of perceived 'high', higher levels of effort to accomplish the driving test, decreased ratings of subjective driving quality, and increased heart rate. There was no strong relationship, within subjects, between plasma levels of the drug and SDLP. This was because drug concentrations declined between the first and second sampling time, whereas SDLP scores hardly changed. When average intra-subject correlations were computed for the first and second sampling times separately, the correlations between [THC] and SDLP were 0.59 $(p<.01)$ and $0.42(p<.05)$, respectively. 


\section{Inter-Subject Relations between Variables}

Relationship between Drug Levels and Performance. Inter-subject correlations between plasma concentrations of the drug and performance were calculated to determine whether subjects with higher plasma levels of the drug performed poorer than those that had lower plasma levels. Correlations between driving performance and performance in the laboratory were also calculated. The results are presented in Table 6.5. It appeared that correlations involving plasma concentrations were greater when logarithmic values of THC values were used in the computation $(1 \mathrm{ng} / \mathrm{ml}$ was added to all THC values before the transformation in order to avoid negative log values). Correlations with $\mathrm{THC}-\mathrm{COOH}$ were generally smaller than those with $\log$ THC values; therefore only correlations with $\log$ THC values are shown in the table.

Table 6.5 Inter-subject correlations between drug concentrations in plasma and raw performance scores in marijuana conditions ( ${ }^{*} p<.05$, ${ }^{* *} p<.01 ; 2$-tailed).

\begin{tabular}{|c|c|c|c|c|c|c|}
\hline \multirow{2}{*}{ 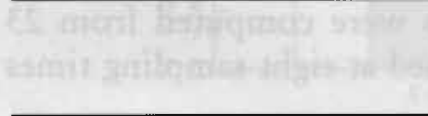 } & \multicolumn{2}{|c|}{$100 \mu \mathrm{g} / \mathrm{kg}$} & \multicolumn{2}{|c|}{$200 \mu \mathrm{g} / \mathrm{kg}$} & \multicolumn{2}{|c|}{$300 \mu \mathrm{g} / \mathrm{kg}$} \\
\hline & $1^{\mathrm{st}}$ & $2^{\text {nd }}$ & $1^{\text {st }}$ & $2^{\text {nd }}$ & $1^{\text {st }}$ & $2^{\text {nd }}$ \\
\hline$r(\log ([\mathrm{THC}]+1), \mathrm{SDLP})$ & -.23 & -.32 & -.26 & +.01 & +.13 & +.07 \\
\hline$r(\log ([\mathrm{THC}]+1), \mathrm{SP})$ & $-.68^{*}$ & $-.72^{\circ 9}$ & $-.45^{\circ}$ & -.39 & -.52 & $-.58^{\circ *}$ \\
\hline$r(\log ([\mathrm{THC}]+1), \mathrm{SDSP})$ & -.37 & $-.57^{*}$ & $-.52^{*}$ & -.38 & -.27 & -.22 \\
\hline$r\left(\log ([\mathrm{TH} H \mathrm{C}]+1), \lambda_{d}\right)$ & +.33 & $+.51^{*}$ & +.24 & +.35 & +.08 & +.33 \\
\hline$r(\log [[\mathrm{THC}]+1)$, side contacts $)$ & +.16 & +.24 & +.04 & -.19 & +.31 & $+.47^{\circ}$ \\
\hline$r\left(\right.$ SDLP,,$\left.\lambda_{\nu}\right)$ & $-.47^{*}$ & $-.50^{\circ}$ & $-.42^{\circ}$ & $-.56^{\circ}$ & $-.47^{\circ}$ & -.27 \\
\hline$r$ (SDLP, side contacts) & +.41 & +.38 & +.10 & +.41 & +.27 & +.38 \\
\hline
\end{tabular}

Table 6.5 shows that SDLP was not related to prevailing plasma levels of THC. Another driving performance measure, mean speed, was only moderately, yet consistently, related to THC; subjects having high [THC] values drove slower than those having low [THC] values. Driving performance was moderately related to critical tracking; poorer tracking performance on the road (higher SDLPs) concurred with poorer tracking performance in the laboratory (lower $\lambda_{c} s$ ).

Five percent of the driving tests undertaken in this experiment yielded SDLP scores above the normal limit of $35 \mathrm{~cm}$ (Table 6.6). This limit was established by several hundred young and middle-aged volunteers and psychiatric patients who uniformly failed to achieve higher scores in the same test after being treated with placebo in all of the Institute's studies since 1986. It is illuminating to examine the present drivers who drove over the limit with respect to their prior treatments and the plasma concentrations of THC and its metabolite they exhibited at these times.

These data are important in two respects. Aberrant driving performance never occurred after placebo smoking, only once after the lowest THC dose and with equal frequency $(4 \mathrm{x})$ after both of the higher doses. Moreover, aberrant driving 
generally occurred during the second and not the first ride in direct opposition to the trend in plasma THC concentrations. From this, it's easy to infer the futility of predicting changes in SDLP, and presumably other aspects of driving performance, from a single [THC] estimation.

Correlations were also computed between drug plasma concentrations and changes in driving performance from placebo to marijuana conditions. These correlations were generally smaller than those involving the raw scores. Thus, [THC] does not predict changes in that performance.

Table 6.6 Drug levels and SDLPs from those subjects whose SDLPs exceeded $36.0 \mathrm{~cm}$.

\begin{tabular}{llllll}
\hline subject & condition & trial & $\begin{array}{l}{[\mathrm{THC}]} \\
(\mathrm{ng} / \mathrm{ml})\end{array}$ & $\begin{array}{l}{[\mathrm{THC}-\mathrm{COOH}]} \\
(\mathrm{ng} / \mathrm{ml})\end{array}$ & $\begin{array}{l}\text { SDLP } \\
(\mathrm{cm})\end{array}$ \\
\hline 1523 & $100 \mu \mathrm{g} / \mathrm{kg}$ & 2 & 2.5 & 3.2 & 38.7 \\
1503 & $200 \mu \mathrm{g} / \mathrm{kg}$ & 2 & 1.7 & 5.1 & 36.3 \\
1523 & $200 \mu \mathrm{g} / \mathrm{kg}$ & 1 & 8.8 & 6.7 & 39.2 \\
1523 & $200 \mu \mathrm{g} / \mathrm{kg}$ & 2 & 4.3 & 4.3 & 37.0 \\
1527 & $200 \mu \mathrm{g} / \mathrm{kg}$ & 2 & 10.0 & 16.2 & 39.4 \\
1506 & $300 \mu \mathrm{g} / \mathrm{kg}$ & 2 & 9.8 & 3.8 & 36.6 \\
1523 & $300 \mu \mathrm{g} / \mathrm{kg}$ & 1 & 18.0 & 11.5 & 36.9 \\
1523 & $300 \mu \mathrm{g} / \mathrm{kg}$ & 2 & 5.2 & 9.2 & 39.3 \\
1526 & $300 \mu \mathrm{g} / \mathrm{kg}$ & 2 & 6.7 & 16.2 & 36.6 \\
\hline
\end{tabular}

Relationship between Driving Performance and Frequency of Current Use. Subjects were classified into two categories according to the frequency of reported cannabis consumption. Twelve subjects were infrequent users; i.e., between once weekly to once monthly; eleven subjects were classified as frequent users; i.e. at least once weekly but less than daily. SDLP values then entered a repeated measures MANOVA with Frequency of use as a between-groups factor, and Dose and Time as within-subjects factors. MANOVA failed to show a main effect of Frequency; interactions of Frequency and the other factors were also not significant.

Relation between Driving Performance and Driving under the Influence Experience. Subjects were classified into two categories according to the frequency of reported driving within one hour of cannabis consumption (see Table 5.1). SDLP values then entered a repeated measures MANOVA with Experience as a betweengroups factor, and Dose and Time as within-subjects factors. Neither Experience nor any interaction was significant.

Relation of Willingness to Drive to Perceived 'bigh' and Driving Performance. The relationship between willingness to drive and changes in SDLP and perceived 'high' was determined as follows. Groups were defined by their willingness to 
drive; i.e., two groups were defined comprising those subjects who would not have driven and those who would, for each combination of condition (4x), sampling time (2x) and urgency of circumstance $(3 x)$, separately. Student's 2-tailed $t$-test for independent means was employed to determine whether the groups had significantly different change scores (drug minus placebo) of SDLP and perceived 'high'. Thus, 18 different t-test were performed for each variable, SDLP and perceived 'high'. Criterion for statistical significance was set at .01 because of the large number of tests.

No significant differences in SDLP change were found between subjects willing and those reluctant to drive. With respect to changes in perceived 'high', only two significant effects were found. Subjects willing to drive under the imagined circumstance $B$ ('important but avoidable') at both sampling times in the $200 \mu \mathrm{g} / \mathrm{kg}$ condition felt less 'high' than those who would not drive. However, this observation is not of major concern, since it was not supported in the other conditions and circumstances.

From these results, it can therefore be concluded that subjects' willingness to drive was not related to either perceived 'high' or driving performance.

\subsection{Discussion}

This study demonstrated that marijuana impairs driving performance as measured by an increase in SDLP; all three THC doses significantly affected SDLP relative to placebo. It is remarkable that driving impairment was about the same after the two higher doses. This cannot be due to a ceiling effect, since greater deterioration in road tracking performance has been found after many prescription drugs (e.g. Robbe et al., 1989) and also high doses of alcohol (Louwerens et al., 1987). One possible explanation for the lack of a clear dose related impairment may be that subjects were able to mitigate the effects of the highest dose by 'trying harder'. Indeed, subjects reported purting more effort in performing the test after smoking the highest THC dose. In other words, subjects tried to overcome the perceived disparity between their actual state and the one required to drive the car efficiently; the higher the THC dose, the greater the disparity between the actual and required states, and the harder they tried to compensate for it.

Though marijuana's adverse effects on SDLP were somewhat smaller in the second test than in the first, no significant interaction was found between the treatments and repetition of the test. This means that the driving performance decrement after smoking marijuana persisted almost undiminished for two hours after smoking.

What is the practical relevance of the size of the effects of marijuana upon lateral position variability? This can be inferred by comparing marijuana's effects to those of alcohol in the same test. As cited above, Louwerens et al. $(1985,1987)$ 
conducted a study to establish the dose-effect relationship of alcohol. Their study resembled the present one in many aspects: they applied exactly the same driving test, the study was performed on a closed road, and involved the participation of 12 male and 12 female volunteers. They were able to derive an empirical equation for predicting the change in SDLP from placebo levels with increasing blood alcohol concentrations. Mean SDLP began to change significantly at blood alcohol concentration of $0.03 \mathrm{~g} \%$ and increases exponentially to the point where the vehicle's lateral motion can no longer be restricted to within lane boundaries (at about $\mathrm{BAC}=0.12-0.15 \mathrm{~g} \%$, on the average). Elevations of the group's mean SDLP in the first and second test after smoking 100,200 and $300 \mu \mathrm{g} / \mathrm{kg}$ are presented in Figure 6.7, but now as their respective BAC equivalent producing the same driving impairment. Two tentative conclusions can be drawn from these data: first, small doses of THC are capable of impairing driving performance; secondly, performance deterioration is only little worse, yet equivalent to BACs over the Dutch legal limit of $0.05 \mathrm{~g} \%$, when much higher doses are smoked.

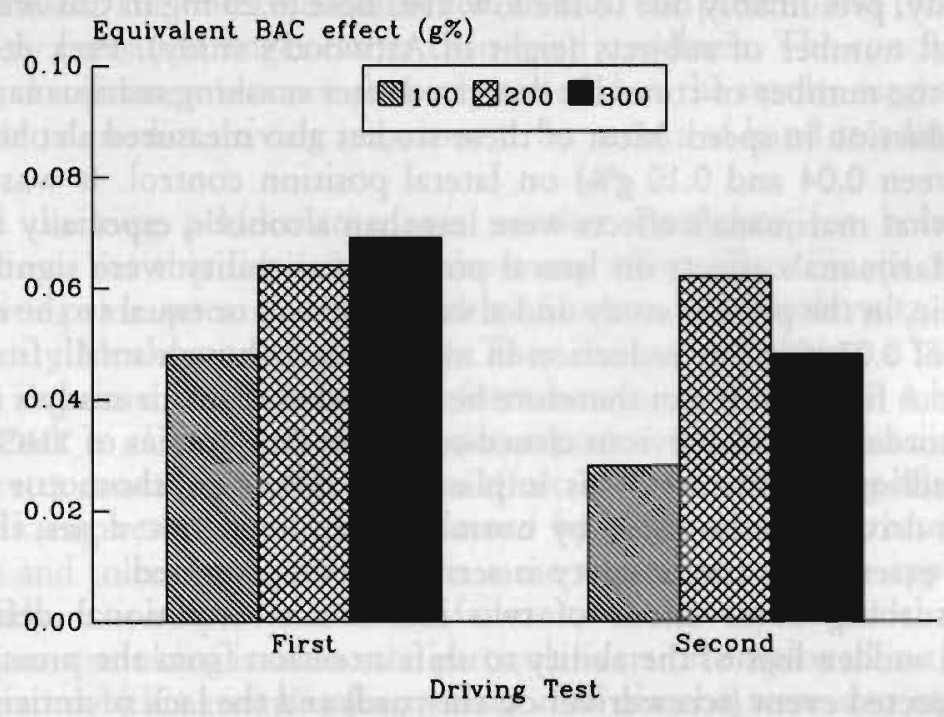

Figure 6.7 Comparison of mean SDLP changes after marijuana smoking to those associated with BAC.

As mentioned above, mean SDLP values did not change greatly over time. The same can be said of mean subjective driving quality ratings. Yet other parameters did change over time; THC in plasma, perceived 'high', heart rate elevation and perceived effort to accomplish the driving test were all less in the second test than in the first. Another important observation was made when the $5 \%$ highest SDLPs were examined in greater detail. Nearly all of these scores were recorded 
in the second rather than the first driving test after smoking higher THC doses. It may be that subjects did not try to compensate as much during the second ride because they felt less intoxicated. Whatever the reason, these results clearly indicate that the behaviorally toxic effect of THC had not yet dissipated. This means that 'behavioral intoxication' may outlast physiological and subjective marijuana intoxication, a phenomenon already reported by other investigators (e.g. Reeve et al., 1983; Yesavage et al., 1985). On the other hand, subjects' perceived driving performance, measured retrospectively, did not change from the first to the second test when compared to placebo. Thus, while they felt less intoxicated in the second test they realized that their driving impairment was still the same as in the first test. Subjective feelings of 'high' should therefore not be equated with subjective feelings of impairment, as is often done.

Some investigators (Klonoff, 1974; Hansteen et al., 1976) found that marijuana impairs lateral position control as measured by number of cones hit in slalom tests. Others (Casswell, 1979; Attwood et al., 1981) did not find any effect of marijuana upon lateral position control as measured in a similar way as in the present study, presumably due to the low THC dose (6.25 mg in Casswell's study) or the small number of subjects (eight in Attwood's study). Peck et al. (1986) found that the number of cones hit decreased after smoking marijuana, probably due to a reduction in speed. Most of these studies also measured alcohol's effects (BACs between 0.04 and $0.10 \mathrm{~g} \%$ ) on lateral position control. It was generally concluded that marijuana's effects were less than alcohol's, especially at BACs of $0.08 \mathrm{~g} \%$. Marijuana's effects on lateral position variability were significant, yet not dramatic, in the present study and always less than or equal to the equivalent BAC effect of $0.07 \mathrm{~g} \%$. The reduction in mean speed, though small, fits also well with previous findings. It can therefore be concluded that this study's results are in close accordance with previous closed-course driving studies of THC effects on vehicle handling parameters. This implies that simple psychomotor functions involved in driving are impaired by normally consumed THC doses, though not to such an extent that traffic safety is seriously compromised.

Yet disturbing observations of two individuals' attentional deficits were observed; a sudden loss of the ability to shift attention from the prescribed task to an unexpected event (screwdriver on the road) and the lack of anticipation for a normal event (end of circuit). Since perception and attention are important aspects of actual driving, these instances may indicate an unusually hazardous property of THC when the drug is consumed shortly before operating a vehicle. Therefore, the revised conclusion must be: what was measured was only moderately affected by THC, but another major deficit may have existed after the highest dose which bears further examination. One can not conclude whether the standard driving test applied in this study measures the most important deficits.

Important practical implications of the study are whether driving performance decrements can be predicted by prevailing plasma concentrations of the drug. 
Though average [THC] and [THC- $\mathrm{COOH}$ ] values were clearly dose-related, driving impairment reached a ceiling before the highest concentrations were achieved. Inter-subject correlations between plasma concentrations of the drug and driving performance after every dose were essentially nil, partly due to the peculiar kinetics of THC. It enters the brain relatively rapidly, although with a perceptible delay relative to plasma concentrations. Once there, it remains even at a time when plasma concentrations approach or reach zero. The conclusion is that driving impairment cannot be predicted by prevailing plasma concentrations of THC or THC-COOH.

Another way of predicting driving impairment was explored; namely, by performance in laboratory tests that might be potential 'roadside' tests. Hand steadiness was impaired by marijuana after high doses but not after $100 \mu \mathrm{g} / \mathrm{kg}$. Performance in this test was not related to driving performance. Previous studies employing the same test (Clark et al., 1970; Milstein et al., 1975) showed greater sensitivity to marijuana-induced impairment of hand steadiness. In those studies subjects were not allowed to rest their hands on the table, which is probably the major reason for the observed difference. In fact, the present and the preceding study merely measured finger rather than hand steadiness. The conclusion must be that hand steadiness was not properly tested, but the results suggest that it is not possible to predict driving impairment by means of hand steadiness performance.

Critical tracking performance was another candidate for being a good 'roadside' test. Yet this test failed to show any effect of marijuana which is in conflict with prior research conducted by Sharma and Moskowitz (1975) and more recently Moskowitz et al. (1981). They demonstrated that a THC dose of $200 \mu \mathrm{g} / \mathrm{kg}$ impairs critical tracking performance for at least up to 4 hours postsmoking. Peck et al. (1986), however, also reported a failure of marijuana alone ( $1.0 \mathrm{~g}$ cigarette containing $1.9 \% \mathrm{THC}$ ) to affect $\lambda_{\mathrm{c}}$ scores. They hypothesized that their conflicting results could be explained by their subjects' greater cannabis experience and tolerance. Although the same argument could be applied in the present study, it is suspected that the failure to detect significant changes in $\lambda_{c}$ after marijuana smoking is due to the particular version employed in this and the laboratory study (this group's mean values after placebo were also considerably lower than those commonly found in healthy volunteers). The device used was a commercial PC/AT version of CTT which was originally programmed on a Commodore-64 computer. The latter has been successfully applied in psychopharmacological research by the authors and their colleagues and hitherto appeared as a very sensitive test for drug-related impairment (e.g. Robbe et al., 1989; Ramaekers et al., 1992a). This study was the first in which the commercial PC/AT version was employed, so the conclusion that the software or hardware was not well designed seems inevitable. 
All subjects were willing to undertake the driving tests. But test conditions were artificial and the same individuals may or may not have been willing to drive under normal circumstances. Therefore, one questionnaire inquired into the subject's willingness to drive an automobile when experiencing the same drug effect under 'normal' conditions. Subjects' willingness to drive was related both to consumed THC dose and urgency. After smoking a low THC dose, nearly all were willing to drive, especially for urgent reasons. After higher doses, fewer were willing to drive under all circumstances. According to what subjects said, they did not become less cautious after inhaling increasingly large doses (unlike what is often reported about alcohol). On the contrary, their caution increased with intoxication. Still, $50 \%$ of the subjects reported that they would have driven for an unimportant reason shortly after smoking the two highest THC doses. Since willingness to drive was not related to objective driving impairment, it may be concluded that at least some of the subjects either were not able to appraise their driving performance before they had actually driven or did not consider their impairment as critical.

It is interesting to compare plasma concentrations of THC found after smoking the highest THC dose in this study with those found in the laboratory study. The highest dose administered in the present study was comparable to the average dose the same subjects consumed in the laboratory study. Mean plasma concentrations of THC after the highest dose in the present study, determined 30 and 90 minutes after initiation of smoking, were 50 and $40 \%$ higher than those measured in the laboratory study after 40 and 100 minutes. This large difference can only be partially explained by the 10 minutes delay between the two studies' blood sampling schedule. Apparently, subjects smoked more efficiently in this than the previous study. Since most volunteers were used to smoking hashish instead of marijuana, the increased efficiency compared to that in the laboratory study may be explained by familiarization with the particular formulation of the drug.

One of the objectives of this study was to determine whether or not the same experimental conditions could be implemented in a study on marijuana's effects on driving on a primary highway public for other traffic. All subjects were willing and able to finish the driving tests without great difficulty. In cases of the exceptional events, the driving instructor was able to control the situation, safely. The effects of marijuana on SDLP were never so large as after many other drugs that had been safely studied in the presence of other traffic. Furthermore, it can be inferred from what subjects said and did that they would not be expected to seek dangerous situations and would be responsive to the instructor's advice. Normal safeguards were deemed sufficient to ensure safety. Hence, the final conclusion was that it would be safe to repeat this study on a normal highway in the presence of other traffic. 


\section{CHAPTER 7}

\section{MARIJUANA AND DRIVING ON A NORMAL HIGHWAY IN TRAFFIC}

\subsection{Introduction}

The preceding study showed that the average driving impairment on a closed highway segment was never particularly great after doses of 100, 200 and $300 \mu \mathrm{g} / \mathrm{kg}$ THC (equivalent to impairment at 0.05 to $0.07 \mathrm{~g} \% \mathrm{BAC}$ ) making it ethically acceptable to test the same doses' effects on performance in a more natural environment. It seemed essential to do so because the extent to which one can generalize from closed-course testing to the real world is presently unknown. Therefore, the same approach was applied for testing THC's effects on driving performance in a more realistic situation. In particular, the same THC doses were administered to a new group of subjects undertaking similar driving tests, though now on a highway in the presence of other traffic.

The present study was only the second wherein subjects drove in real traffic after receiving THC treatments. It was the first in which subjects inhaled higher 'street doses' of THC before driving. Though the preceding study on a closed highway failed to show dramatic effects of high THC doses on high-speed driving, a conservative approach was chosen in designing the present study in order to satisfy the strictest safety requirements. This approach is unique in traffic science but closely follows the procedure used to determine the tolerability of medicinal drugs in human pharmacological research. It is to test THC's effects on actual driving performance in an ascending dose series (below). If any subject would have reacted in an unacceptable manner to a lower dose, he/she would not have been permitted to receive a higher dose.

The major objective of this study was to confirm the relationship between inhaled THC dose and lateral position variability in the context of a standard road tracking test. A secondary objective was to measure performance in another actual driving test (i.e. car following) to determine whether degrees of impairment would correlate between the two tests in a manner indicating a general influence of THC on driving behavior. The third objective was to continue efforts to correlate plasma concentrations of THC and THC-COOH with driving performance impairment as measured in both tests. 


\subsection{Methods}

\section{Subjects}

Sixteen new subjects, equally comprised of men and women, were selected according to the same inclusion/exclusion criteria as before. They were individually trained to perform the driving tests in a preliminary 'dress rehearsal'. Training at laboratory tests continued until each subject achieved satisfactory, asymptotical performance levels.

Plasma analyses after conclusion of the study showed that one female's plasma contained neither THC nor THC-COOH in any sample. It was concluded that this subject had not inhaled smoke, so her data were excluded from further analyses. Characteristics of the remaining 15 subjects are given in Table 7.1. T-tests for independent samples showed that males were heavier smokes than females $(p<.007)$. There were no other significant differences between the sexes.

Table 7.1 Mean \pm SD (range) of subjects' characteristics.

\begin{tabular}{lll}
\hline & $\begin{array}{l}\text { males } \\
(\mathrm{N}=8)\end{array}$ & $\begin{array}{l}\text { females } \\
(\mathrm{N}=7)\end{array}$ \\
\hline age (yrs) & $28.3 \pm 7.4(22-42)$ & $25.0 \pm 4.6(21-34)$ \\
weight $(\mathrm{kg})$ & $70.8 \pm 7.0(61.0-83.5)$ & $66.7 \pm 7.9(55.5-79.0)$ \\
weight $(\mathrm{lb})$ & $156 \pm 15(134-184)$ & $147 \pm 17(122-174)$ \\
smoking experience (yrs) & $8.3 \pm 6.5(2-21)$ & $6.3 \pm 5.9(1-16)$ \\
\# joints/month & $8.3 \pm 5.0(1-16)$ & $2.0 \pm 1.4(1-4)$ \\
driving experience (yrs) & $6.8 \pm 5.7(2-20)$ & $4.9 \pm 4.6(1-15)$ \\
driving experience (km x 1000) & $78 \pm 101(10-320)$ & $38 \pm 66(5-188)$ \\
driving experience (mi x 1000) & $48 \pm 63(6-199)$ & $24 \pm 41(3-117)$ \\
\# of subjects having driven within & & \\
1 hr following cannabis consumption: & & \\
less than 5 times & 4 & 0 \\
5 times or more & 4 & \\
\hline
\end{tabular}

\section{Design, Doses and Administration}

The study was conducted according to an ascending dose series design where both active drug and placebo conditions were administered, double-blind, at each of three THC dose levels. THC doses were the same as those used in the previous study, namely 100,200 and $300 \mu \mathrm{g} / \mathrm{kg}$.

The lowest dose and placebo were administered in separate First Level treatment conditions spaced a week apart. Half the group received these treatments in the same order, the others in reverse order. Subjects proceeded to the next dose only if, in the driving instructor's opinion, no severe and potentially unsafe drug effects had occurred; and, if the subject was willing to go on to the next level. The intermediate dose and placebo were administered the same way 
in the Second Level conditions and the decision to proceed was made on the same grounds. The highest dose and placebo were administered the same way in the Third Level conditions to conclude the study.

Marijuana cigarettes were prepared from batches containing $1.77 \%$ THC for the lowest, $2.64 \%$ THC for the intermediate, and $3.58 \%$ THC for the highest dose. Cigarettes appeared identical at each level of treatment conditions. Subjects smoked the cigarettes through a plastic holder, ad lib, but they were encouraged to smoke the entire dose in ten minutes. After cessation of smoking, cigarettes were retained for subsequent gravimetric estimation of THC consumed. These analyses revealed that the average $( \pm S D)$ amount of consumed THC in the three marijuana conditions was $6.9( \pm 0.7), 13.8( \pm 1.4)$ and $20.7( \pm 2.2) \mathrm{mg}$, or $100( \pm 4)$, $204( \pm 7)$ and $299( \pm 7) \mu \mathrm{g} / \mathrm{kg}$, respectively.

\section{Testing Procedures}

Upon arrival at the laboratory, subjects' breath and urine were tested for the presence of alcohol and cannabinoids. If cannabinoids were found in the urine, a blood sample was taken for later verification of the presence of THC. Two subjects at a time commenced smoking at $t=0$ (Table 7.2).

Table 7.2 Schedule of activities on test-days.

\begin{tabular}{ll}
\hline relative time (min) & activity \\
\hline $0-10$ & smoking \\
$30-40$ & tracking, hand steadiness and body sway tests \\
$40-45$ & blood sampling \\
$55-70$ & car following test (1st subject) \\
$70-85$ & car following test (2nd subject) \\
$85-135$ & standard driving test (both subjects) \\
$140-155$ & car following test (1st subject) \\
$155-170$ & car following test (2nd subject) \\
$190-195$ & blood sampling \\
$195-205$ & tracking, hand steadiness and body sway tests \\
\hline
\end{tabular}

Thirty minutes after onset of smoking the subjects performed a battery of laboratory tests (tracking, hand steadiness and body sway) and yielded a blood sample. They were then transported to a primary highway (A76, different than in the previous study) between the Dutch cities of Maastricht and Heerlen where the driving tests were performed. Two instrumented vehicles, the same as those in the previous study, were employed in this study. One subject started the car following test (below) in the eastward direction whilst the other subject was sitting in the passenger's seat of the preceding car involved in the same test. The test was conducted on a $16 \mathrm{~km}(9.9 \mathrm{mi})$ circuit of the highway and lasted about twelve minutes. At the end of the circuit the car turned at a signalized intersec- 
tion and parked at a service station, whereupon the subjects reversed roles to repeat the test running in the opposite direction. The new driver reentered the highway and began his/her car following test. After conclusion of the car following test, the subject left the highway at an exit ramp and reentered in the opposite direction on the associated entrance ramp. Thereupon both vehicles parked on the paved shoulder.

Both subjects then commenced the standard driving test (below) in separate instrumented vehicles at $t=85$ and $t=88$, respectively. The test circuit was the same as for the car following test. Subjects drove twice around the circuit (in total, $64 \mathrm{~km}$ or $40 \mathrm{mi}$ ) without stopping in about 50 minutes. At the conclusion of this test, both subjects participated again in the car following test in the same order as before. Subjects were then transported back to the laboratory where they yielded a blood sample and repeated the test battery.

Two pairs of subjects were tested per test night. One pair performed the driving test in daylight, i.e. between 19.30 and 21.30 hours; the other pair commenced driving at 21.30 hours and finished at 23.30 hours in darkness.

\section{Driving Tests}

The standard test was the same as described in the previous study (Chapter 6) except for its duration and the presence of other traffic. Subjects were instructed to maintain a constant speed of $95 \mathrm{~km} / \mathrm{h}(59 \mathrm{mph})$ and a steady lateral position between lane boundaries in the right traffic lane. They were allowed to deviate from this only if it would become necessary to pass a slower vehicle in the same lane. Data from the standard test were analyzed to yield the same performance measures as in the previous study; namely, standard deviation of lateral position (SDLP), mean and standard deviation of speed (SP and SDSP), and standard deviation of steering wheel angle (SDST).

The car following test has been applied previously in a preliminary version by Brookhuis et al. (1987) and measures the driver's ability to perceive changes in a preceding vehicle's speed and to react in a manner maintaining a constant headway. It began as the preceding and the following vehicle, respectively driven by one of the driving instructors and the subject, operated in tandem on the slower traffic lane while travelling at a speed of $100 \mathrm{~km} / \mathrm{h}(62 \mathrm{mph})$. The subject was instructed to maintain a $50 \mathrm{~m}$ (164 ft) headway however the preceding vehicle's speed might vary. After driving in this manner for about one minute, the operator of the preceding vehicle released the accelerator pedal allowing its speed to fall to $80 \mathrm{~km} / \mathrm{h}(50 \mathrm{mph})$. Immediately thereafter, the operator of the preceding vehicle accelerated to $100 \mathrm{~km} / \mathrm{h}(62 \mathrm{mph})$. The duration of one deceleration and acceleration maneuver was approximately 50 seconds and six to eight, depending upon traffic density, were executed during one test.

The velocity of the leading vehicle was transmitted via telemetry to a receiver in the following vehicle. This signal, along with the following vehicle's own 
velocity were recorded in parallel, time-coded files on computer files. These data entered a power spectral analysis for yielding phase-delay, modulus or gain and coherence between the vehicle's velocities at the maneuver cycle frequency (i.e. $1 / 50 s=0.02 \mathrm{~Hz}$ ). The average phase-delay between frequencies of 0.01 and 0.03 $\mathrm{Hz}$, encompassing the frequency of the deceleration and acceleration maneuvers, was then calculated and transformed to the time domain to yield a measure of the subject's average reaction time to the movements of the leading vehicle (RT, in seconds). This was taken as the primary dependent variable from the car following test. Gain and coherence were recorded for control purposes. If the test was performed according to instructions, gain should have a value of about 1.0 , and coherence, $>.90$.

During the trials, 3 to 6 direct measurements of separation distance between the following and leading vehicle were made by means of a S-VHS video recording system which was mounted between the following vehicle's front seats facing forward through the windshield. Images of the rear of the preceding vehicle were acquired prior to each deceleration/acceleration maneuver. The camera's internal clock signal was recorded with the video imagery and also converted in an electronic pulse code for simultaneously computer recording along with the two vehicles' speed.

Video imagery recorded throughout the trials were analyzed off-line using an interactive software routine implemented on a IBM-AT computer. A single frame would show the appearance of the preceding vehicle at the moment it begins to decelerate. Next the coordinates of two target markers, spaced $119 \mathrm{~cm}$ apart on the rear of the leading vehicle were identified on the display. From this information the distance separating the two vehicles or 'headway' was calculated according to the equation,

$$
\mathrm{d}(\text { in } \mathrm{m})=\mathrm{k}(1 \mathrm{~m} / \tan (\Theta / 2))
$$

where $\mathrm{k}$ is a proportionally constant and $\theta$ the horizontal angle subtended by the camera lens. Once the starting distance was determined from a single measurement, headway changes during the maneuver were calculated using differential speed according to the equation,

$$
H(t)=\int V_{p} d t-\int V_{f} d t+H_{0}
$$

where headway varies as a function of time $(t)$ according to the difference between integrals of velocities of preceding and following vehicles $\left(V_{p}, V_{i}\right)$ plus the headway $\left(\mathrm{H}_{0}\right)$ that existed at the beginning of the maneuver. Headway and coefficient of variation (CV) of headway during maneuvers were taken as secondary dependent variables. The coefficient of variation of headway, and not standard deviation, is preferable because of the latter's confounding by mean headway. 


\section{Questionnaires}

The same subjective questionnaires used in the previous study were administered to the subjects in the present study. Subjective feelings of 'high', present cognitive and emotional state, and subjects' willingness to drive were assessed before the onset and after the conclusion of the driving tests ( $t=50$ and 175). Subjects were also asked about their perception of the administered treatment, whether it was THC or placebo. At the end of each driving test, subjects were required to retrospectively rate the effort given in performing the test and perceived driving quality.

\section{Laboratory Tests}

Three tests were administered to the subjects: critical tracking, hand steadiness and body sway. These were also administered in the preceding studies, but the equipment or procedures were changed in this study.

1. Equipment, not procedures, changed in case of the critical tracking test (CTT). The test had shown no sensitivity to treatments administered in the preceding studies, in spite of the fact that other investigators had used the same test for showing significant effects of much lower THC doses. The validity of the particular MS-DOS version used in the preceding studies was doubtful. Therefore an older Commodore-64 version was employed in the present study. This version of the CTT had proven its sensitivity to drug-induced sedation in several previous studies conducted by the authors and their colleagues (e.g. Robbe et al., 1989; Ramaekers et al., 1992a). Test duration was approximately 5 minutes and mean $\lambda_{c}$ was the dependent variable.

2. As discussed in the previous chapter, the hand steadiness test examined finger rather than hand steadiness because subjects were allowed to rest their hands on the table. Subjects were not allowed to do this in the present study. The modification was expected to induce greater instability. Therefore, the diameters of holes were increased to avoid ceiling effects of the number of contacts between their sides and the hand-held stylus. The diminishing diameters of the five circular holes were now set to $6.30,4.70,3.90,3.05$ and $2.70 \mathrm{~mm}(0.248,0.185,0.154,0.120$ and $0.106 \mathrm{in})$ respectively. The dependent variable was again the square root of the total number of contacts of the stylus with any side. The test lasted about 3 minutes.

3. Postural instability, or body sway, was measured using the stabilometry method (Kapteyn et al., 1983). It involved the use of a balance platform that measures the location of the vector of force which extends vertically downward from the body's center of gravity and its movement over time. Analog output of force transducers within the platform were digitized and analyzed to yield simultaneous measures of lateral and sagittal motion around the vertical axis. Subjects were instructed to maintain a static posture while standing over the center of the balance platform with their feet together. 
Two, 30-second recordings followed. The first with the subject's eyes open, the second with eyes closed. While standing with the eyes open, the subject was required to fixate on a target mounted on the wall from a distance of 2.0 $m(6.6 \mathrm{ft})$. The mean area circumscribed by the vertical vector of force (i.e. curve surface, in $\mathrm{mm}^{2}$ ) was taken as the dependent variable (CS-O and CS-C for eyes open and closed, respectively).

\section{Physiological Assessments}

The electrocardiogram (ECG) was measured from precordial leads $\left(\mathrm{RC}_{5}\right)$, and the interbeat interval (IBI) times were registered continuously during the driving tests. Cardiac interval times were analyzed to yield three different parameters, mean IBI, the coefficient of variation (CV-IBI), and a spectral measure of heart rate variability (PWR-HR). CV-IBI, is defined as standard deviation of IBI divided by the mean. It is the best measure of IBI variability in the time domain. The coefficient of variation, and not the standard deviation, of IBI is preferable because of the latter's confounding by mean IBI. PWR-HR is a spectral measure of heart rate variability and calculated from the time series of instantaneous heart rates, which are computed at each successive heart beat. Spectral variability is expressed as the 'squared modulation index' (Van Dellen et al., 1985) which is comparable to the squared coefficient of variation in the time domain if it is integrated over the whole spectrum $(0.01-0.50 \mathrm{~Hz})$. PWR-HR is defined as the integration of spectral density values between 0.07 and $0.14 \mathrm{~Hz}$.

Mean interbeat interval, and more frequently its reciprocal, mean heart rate, is frequently used for measuring THC's chronotropic cardiac effect. As shown in the previous study, the measure is valuable for assessing the course of the drug's activity over time. However the other measures, CV-IBI and PWR-HR, possess greater psychological significance. They are alternatively used for estimating relative changes in an individual's mental workload, or more correctly the amount of mental effort he must exert for handling a particular workload. In general, both measures vary inversely with the imposed mental workload or the increase in mental effort which is required to cope with a constant workload while maintaining the same level of performance efficiency under the influence of drugs or fatigue (Mulder, 1980; Mulder and Mulder, 1981).

\section{Blood Sampling}

Blood samples were obtained from the subjects by venepuncture immediately before they were transported to the test site and immediately after their return, or approximately 35 and 190 minutes after initiation of marijuana smoking. Two aliquots containing $10 \mathrm{ml}$ each were heparinized and centrifuged, and the plasma fractions were placed in frozen storage for later assays to determine [THC] and [THC-COOH]. The analytical procedures were the same as those employed in the preceding studies. 


\section{Data Analysis}

The first step executed was determining the reliability and consistency of performance and subjective parameters measured in the successive placebo treatment conditions. Test-retest reliability coefficients were determined and mean differences between data collected in successive conditions were tested for significance using repeated measures MANOVA. Previous studies wherein unmedicated subjects performance was repeatedly measured in the standard test have failed to show any significant changes over time intervals as long as one week; and, test-retest reliability coefficients for the SDLP measure have always been higher than $r=.80$. However, intervals separating successive placebo tests were longer in the present study and the reliabilities of measures obtained in the car following test had yet to be determined. For these reasons it appeared necessary to check the consistency of the subjects' performance in the present study.

Subsequently, data from drug and placebo conditions on the first, second and third levels were analyzed separately. Performance variables recorded on ratio or interval scales were subjected to repeated measures MANOVA with $\operatorname{Sex}$ as a between-groups and Drug (placebo versus marijuana) as a within-subjects factor. The effect of Time of testing (2 levels) was, if pertinent, simultaneously tested as a within-subjects factor in the same analyses.

It appeared that marijuana's effects on reaction time in the car following test were confounded by mean headway. Reaction times were therefore also analyzed by means of covariance analysis, using headway as the covariate. Covariance analysis estimates what the scores on one variable (in this case, reaction time) would have been if the same 'average' score on another variable (in this case, mean headway) would have occurred in all conditions. It allows one to estimate the effects of THC on reaction time independently of those on headway.

Willingness to drive data were analyzed in the same manner as the parametric data. That is, data from drug and placebo conditions on the first, second and third levels were analyzed separately. Thus, data from both sampling times in a drug condition and its respective placebo condition were simultaneously tested for assessing differences in the proportion of subjects willing to drive for a particular reason.

Separate dose effects were tested using the 'Sequential Bonferroni' procedure for adjusting the $\alpha$-probability criterion $(p)$ in accordance with the number of separate comparisons in a given set (Overall and Rhoades, 1987). For the largest of three differences tested at once, $p$ had to be less than $.05 / 3=.017$ to be judged significant. For the second, it had to be $<.05 / 2=.025$; and for the smallest difference, $p<.05$. The adjustment had the effect of holding the probability of making a type-I error at $p \leq .05$ over the entire set of comparisons.

Though data from both the marijuana and respective placebo condition entered MANOVA, figures illustrating the results display mean difference scores 
(drug-placebo) and standard errors of the difference (SED). The former are depicted by the height of the bars, the latter by the vertical lines above or below the bars. Difference scores were used in order to keep the figures as simple as possible. As a consequence, main effects of Time could not be depicted in the figures: a parallel rise or fall in the mean levels of a variable over time would not affect their difference. This is, however, not a major problem, since it is not very interesting to know whether subjects' performance changed in both conditions in the same manner. More interesting is a Time by Drug effect, which means that the difference between effects of marijuana and placebo changed over time. This effect would be obvious in the figures.

Significant Sex effects were generally absent. Results pertaining to differences between the sexes are therefore mentioned only in the exceptional cases where these were significant.

\subsection{Results}

No subject dropped out during the experiment, neither on their own initiative nor on the driving instructor's. Therefore, the results presented below include data from fifteen subjects at each level of treatment. It was impossible to obtain every blood sample from one woman, even after repeated attempts. Her data were therefore excluded from all analyses involving drug and metabolite plasma concentrations. However, assays of what samples were obtained indicated that she did inhale THC. Consequently, her data were not excluded from other analyses.

\section{Test-Retest Correlations and Consistency of Performance}

Correlations between measurements obtained from the successive placebo treatment conditions are shown in Table 7.3. Correlations between repeated SDLP's were somewhat lower than those obtained in earlier studies for 'normal' subjects, but were still both highly significant and consistent. Mean and standard deviation of speed were also quite reliable. Steering wheel variability, however, was not a reliable measure.

Subjective driving quality ratings were also unreliable, probably due to restriction of range; i.e. most subjects naturally rated their driving performance as about normal (100\%) following placebo treatment. Perceived effort ratings were somewhat more reliable, indicating that driving under the conditions of this study was consistently a more effortful occupation for some subjects than others.

Car following parameters were not as reliable as those measured in the standard driving test. Performance in the second ride in the first placebo condition was not related to that in the second ride of the second and third placebo conditions. This means that the test still needs further standardization to reduce 
the error variance of the measures tested. Ratings of perceived effort in the car following test were only slightly more reliable than those obtained in the standard test. Remarkably, ratings of subjective driving quality were highly reliable in the first car following test, but not in the second.

Table 7.3 Correlations between parameters measured at the same time in the successive placebo treatment conditions $(* p<.05 * * *<.01$; one-tailed).

\begin{tabular}{lllll}
$1^{\text {st }}$ and $2^{\text {nd }}$ placebo & $\frac{2^{\text {nd }} \text { and } 3^{\text {rd }} \text { placebo }}{1^{\text {st }} \text { test }} \quad 2^{\text {nd }}$ test & $1^{\text {st }}$ test $\quad 2^{\text {nd }}$ test & $1^{\text {st }}$ test $3^{\text {rd }}$ placebo & $2^{\text {nd }}$ test \\
\hline
\end{tabular}

\begin{tabular}{|c|c|c|c|c|c|c|}
\hline \multicolumn{7}{|l|}{ Standard Driving Test: } \\
\hline SDLP & $+.75^{* *}$ & & $+.76^{* *}$ & & $+.72^{* *}$ & \\
\hline SP & $+.61^{*}$ & & $+.84^{* \prime}$ & & $+.77^{\circ *}$ & \\
\hline SDSP & $+.66^{* *}$ & & $+.69^{* *}$ & & $+.55^{*}$ & \\
\hline SDST & -.21 & & +.17 & & -.62 & \\
\hline $\begin{array}{l}\text { perceived driving quality } \\
\text { perceived effort }\end{array}$ & $\begin{array}{l}+.24 \\
+.72 *\end{array}$ & & $\begin{array}{l}+.24 \\
+.50^{*}\end{array}$ & & $\begin{array}{l}+.30 \\
+.56^{\circ}\end{array}$ & \\
\hline $\begin{array}{l}\text { Car Following Test: } \\
\text { headway }\end{array}$ & $+.73^{* *}$ & +.31 & $+.60^{* *}$ & $+.90^{* *}$ & $+.67^{* *}$ & \\
\hline CV headway & +.48 & +.19 & +.36 & +.40 & $+.55^{\circ}$ & +.07 \\
\hline $\mathrm{RT}$ & $+.61^{*}$ & $+.45^{\circ}$ & $+.81^{*}$ & $+.68^{* *}$ & $+.64^{* *}$ & +.09 \\
\hline perceived driving quality & $+.51^{\circ}$ & +.06 & $+.64^{* *}$ & +.28 & $+.61^{* *}$ & -.06 \\
\hline $\begin{array}{l}\text { effort } \\
y \text { Tests: }\end{array}$ & $+.72^{* *}$ & $+.80^{\circ \prime}$ & $+.67^{* \circ}$ & $+.69^{\circ *}$ & +.33 & $+.71^{\circ}$ \\
\hline$\lambda_{c}$ & $+.68^{* *}$ & $+.70^{\circ *}$ & $+.73^{* *}$ & $+.87^{* *}$ & $+.67^{* *}$ & $+.78^{* *}$ \\
\hline no. of side contacts & $+.75^{\circ *}$ & $+.80^{\prime \prime *}$ & $+.84^{*}$ & $+.66^{\circ *}$ & $+.63^{* *}$ & $+.63^{* *}$ \\
\hline CS-O & +.43 & $+.49^{\circ}$ & +.20 & $+.62^{* *}$ & +.04 & +.31 \\
\hline $\mathrm{CS}-\mathrm{C}$ & +.25 & $+.56^{*}$ & $+.71^{* *}$ & +.18 & +.37 & +.41 \\
\hline $\begin{array}{l}\text { Other Subjective Feelings: } \\
\text { alertness }\end{array}$ & +.44 & & & & $+.59^{\circ}$ & \\
\hline dness & $\begin{array}{l}+.57 \\
+.52^{\circ}\end{array}$ & $+.79^{* *}$ & $+.76^{\circ}$ & $+.77^{\circ}$ & $+.46^{\circ}$ & $+.59^{\circ}$ \\
\hline calmness & $+.71^{* *}$ & +.34 & $+.87^{* *}$ & +.41 & $+.51^{\circ}$ & +.22 \\
\hline
\end{tabular}

Hand steadiness and $\lambda_{c}$, but not body sway measures, were highly reliable. Ratings of alertness, contentedness and calmness were moderately reliable. Surprisingly, alertness and contentedness ratings were generally more reliable when measured after driving, in contrast to calmness ratings which were more reliable before the driving tests commenced. Correlations between perceived ratings of 'high' were not computed since they were generally very low or zero following placebo.

Mean differences between data collected in successive placebo treatment conditions were tested for significance using repeated measures MANOVA. Only $\lambda_{c}$ changed nearly significantly over successive placebo treatment conditions $\left(\mathrm{F}_{2,12}=3.81 ; p<.052\right)$; subjects' performance in this test improved during the study $\left(\lambda_{c}=4.6,4.8 \& 5.0\right.$, for the respective placebo conditions, averaged across both 
sampling times). Obviously performance had not reached an asymptotic level during practice trials which preceded the beginning of experimental sessions.

\section{Plasma Concentrations of the Drug}

Table 7.4 shows mean, median and range of [THC] and [THC-COOH] by Dose and Time. Placebo values were not used in the statistical analyses since these were zero in most cases. Therefore, data from all THC conditions were analyzed in one MANOVA. The tabular data indicate that [THC] was related to inhaled THC dose, and fell to about the same level three hours after smoking.

Table 7.4 Mean, median and range of [THC] and [THC-COOH] in $\mathrm{ng} / \mathrm{ml}(\mathrm{N}=14)$.

\begin{tabular}{|c|c|c|c|c|c|c|c|}
\hline & & \multicolumn{2}{|c|}{$100 \mu \mathrm{g} / \mathrm{kg}$} & \multicolumn{2}{|c|}{$200 \mu \mathrm{g} / \mathrm{kg}$} & \multicolumn{2}{|c|}{$300 \mu \mathrm{g} / \mathrm{kg}$} \\
\hline & & $t=35$ & $t=190$ & $t=35$ & $t=190$ & $t=35$ & $t=190$ \\
\hline$[\mathrm{THC}]$ & $\begin{array}{l}\text { mean } \\
\text { median } \\
\text { range }\end{array}$ & $\begin{array}{l}7.9 \\
6.5 \\
0.8-17.2\end{array}$ & $\begin{array}{l}0.7 \\
0.9 \\
0.0-1.3\end{array}$ & $\begin{array}{l}12.0 \\
10.0 \\
1.5-27.1\end{array}$ & $\begin{array}{l}1.1 \\
1.0 \\
0.0-2.7\end{array}$ & $\begin{array}{l}16.1 \\
15.8 \\
4.7-30.9\end{array}$ & $\begin{array}{l}1.5 \\
1.5 \\
0.4-3.2\end{array}$ \\
\hline$[\mathrm{THC}-\mathrm{COOH}]$ & $\begin{array}{l}\text { mean } \\
\text { median } \\
\text { range }\end{array}$ & $\begin{array}{l}8.2 \\
7.4 \\
1.4-19.4\end{array}$ & $\begin{array}{l}4.1 \\
4.1 \\
0.0-12.0\end{array}$ & $\begin{array}{l}12.2 \\
11.2 \\
2.0-37.2 \\
\end{array}$ & $\begin{array}{l}7.61 \\
6.4 \\
0.0-32.2 \\
\end{array}$ & $\begin{array}{l}15.3 \\
13.0 \\
4.2-39.6 \\
\end{array}$ & $\begin{array}{l}10.0 \\
8.2 \\
1.5-36.3\end{array}$ \\
\hline
\end{tabular}

MANOVA confirmed this impression with significant Dose $\left(\mathrm{F}_{2,11}=20.75 ; p<.001\right)$ and Time $\left(\mathrm{F}_{1,12}=54.81 ; p<.001\right)$ effects, and Dose by Time interaction $\left(\mathrm{F}_{2,11}=17.80\right.$; $p<.001)$. Males had somewhat higher $[\mathrm{THC}]$ than females and the Sex effect approached significance $\left(\mathrm{F}_{1,12}=4.60 ; p<.053\right)$; Sex by Dose interaction was not significant.

Plasma levels of the metabolite, THC- $\mathrm{COOH}$, were about the same as those of THC 35 minutes after initiation of smoking, but did not decline as rapidly. Table 7.4 shows that [THC-COOH] was also dose-related at both sampling times. Significant Dose $\left(\mathrm{F}_{2,11}=14.49 ; p<.001\right)$ and Time $\left(\mathrm{F}_{1,12}=62.50 ; p<.001\right)$ effects, but no Dose by Time interaction, were shown by MANOVA.

\section{Perceived 'high'}

Ratings of 'high' after placebo were near zero in most cases. Therefore changes occurring after marijuana smoking, relative to placebo, instead of the raw values, were analyzed by MANOVA. Average subjective feelings of 'high' were doserelated and greatest just before the first driving test (Figure 7.1). Relative to maximum personal experience, peak levels of intoxication were about $30 \%, 50 \%$ and $75 \%$ after 100,200 and $300 \mu \mathrm{g} / \mathrm{kg}$ doses. The approximately linear correspon- 
dence between the administered dose and average subjective response was impressive.

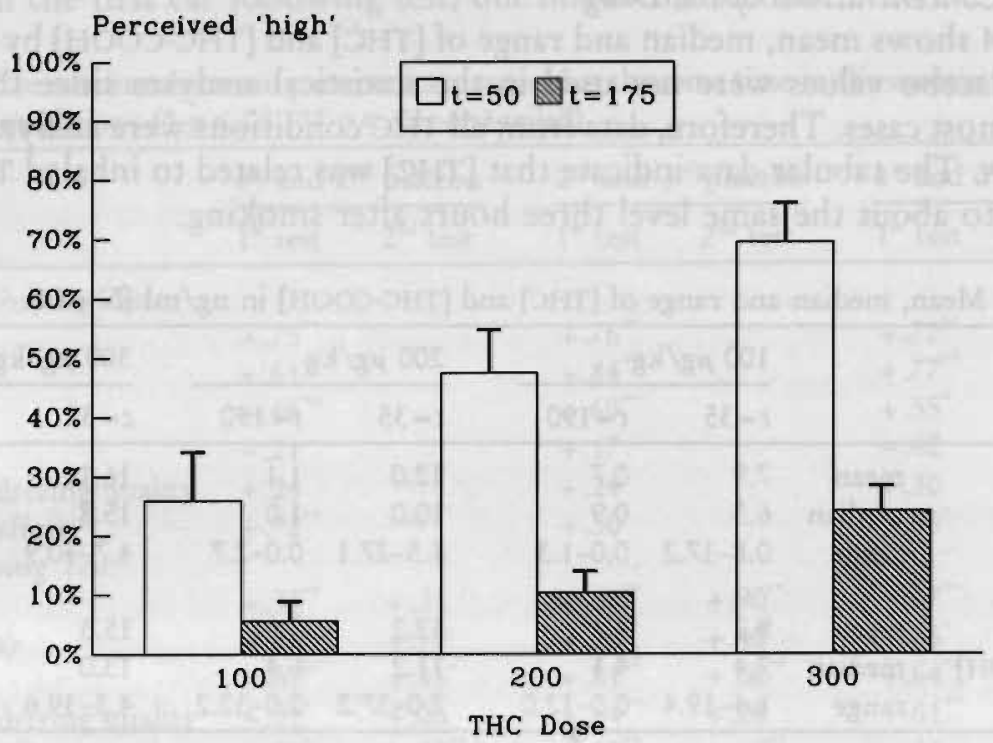

Figure 7.1 Mean (+SED) changes in perceived 'high' by Dose and Time, relative to placebo.

Change scores were significantly dose-related $\left(\mathrm{F}_{2,12}=35.07 ; p<.001\right)$ and timerelated $\left(\mathrm{F}_{1,13}=40.25 ; p<.001\right)$. The decline in feelings of 'high' over time was also dose-related, resulting in a significant Dose by Time interaction $\left(\mathrm{F}_{2,12}=7.87\right.$; $p<.007)$.

Driving Performance in the Standard Driving Test

All subjects undertook and completed each test in a safe manner; the driving instructors neither terminated any ride prematurely nor intervened while subjects were driving. In other words, the subjects' safety was never compromised.

Figure 7.2 shows the mean changes in SDLP from placebo to marijuana conditions. Performance after consuming THC was worse than after the respective placebo treatment; mean changes in SDLP were $1.1,1.8$ and $2.9 \mathrm{~cm}$ for the 100 , 200 and $300 \mu \mathrm{g} / \mathrm{kg}$ conditions, respectively. MANOVA showed that SDLP was significantly elevated after inhaling both the higher, but not the lowest, THC doses relative to placebo $\left(F_{1,13}=2.66,6.63 \& 10.16\right.$ for the 100,200 and $300 \mu \mathrm{g} / \mathrm{kg}$ conditions, respectively; $\left.p<.13, .023 \& .007 ; p_{c}=.05, .025 \& .017\right)$. No significant differences between males and females were discovered. 


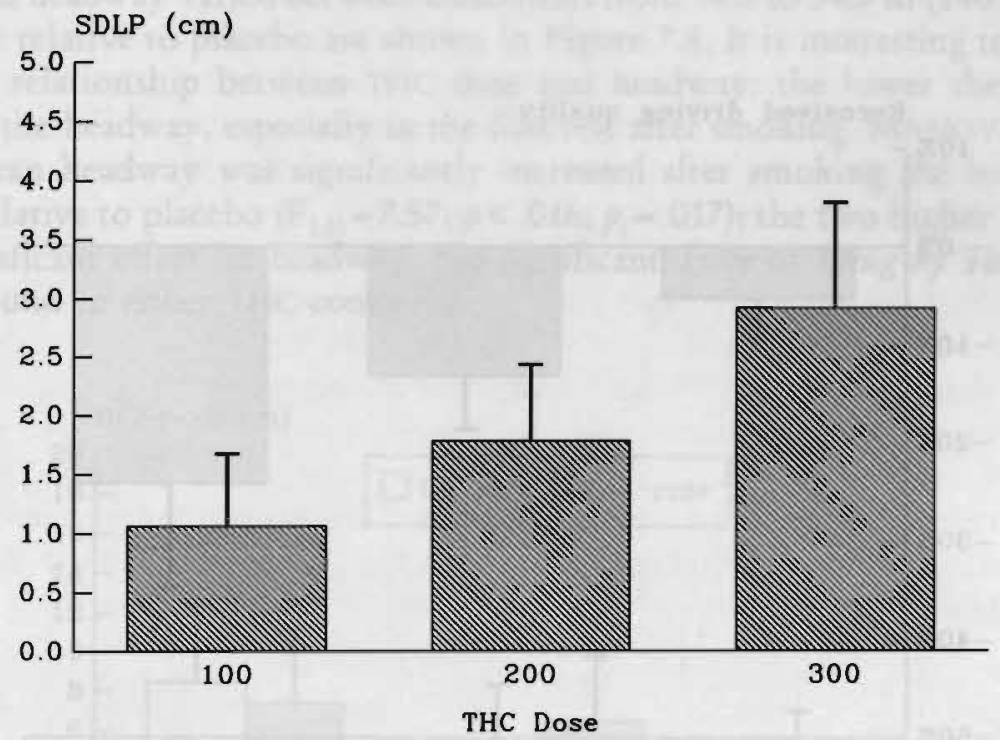

Figure 7.2 Mean (+SED) changes in SDLP in the standard driving test by Drug, relative to placebo.

Mean speed was very close to that established as the target by instructions and varied between conditions from 94.5 to $96.1 \mathrm{~km} / \mathrm{h}$ (58.7 to $59.7 \mathrm{mph})$. Subjects drove slower following marijuana than following placebo, but the mean differences were quite small: $0.3,1.1$ and $0.5 \mathrm{~km} / \mathrm{h}(0.2,0.7$ and $0.3 \mathrm{mph})$ for 100,200 and $300 \mu \mathrm{g} / \mathrm{kg}$ conditions, respectively. MANOVA showed that only the change after the intermediate dose was significant $\left(F_{1,13}=8.05 ; p<.014 ; p_{c}=.017\right)$.

Standard deviation of speed was very small in each condition, on average $2.5 \%$ of mean speed. Though speed variability increased after smoking THC, the effects were minor and not significant. Standard deviations of steering wheel movements were also not affected by THC; mean changes after smoking THC were essentially nil.

Subjects rated their driving performance in the standard test as about 'normal' after smoking placebo and after the lowest dose of THC. Ratings were $90 \%$ and $77 \%$ of 'normal' driving performance following the 200 and $300 \mu \mathrm{g} / \mathrm{kg}$ conditions, respectively. Changes relative to placebo are shown in Figure 7.3. MANOVA demonstrated that changes in perceived driving quality approached the levels of significance required by the 'Bonferroni' adjustment after the two higher THC doses $\left(F_{1,13}=5.29 \& 5.42\right.$ for the 200 and $300 \mu \mathrm{g} / \mathrm{kg}$ conditions, respectively; 
$\left.p<.039 \& .037 ; p_{c}=.025 \& .017\right)$, but were not significantly different from placebo after the lowest THC dose.

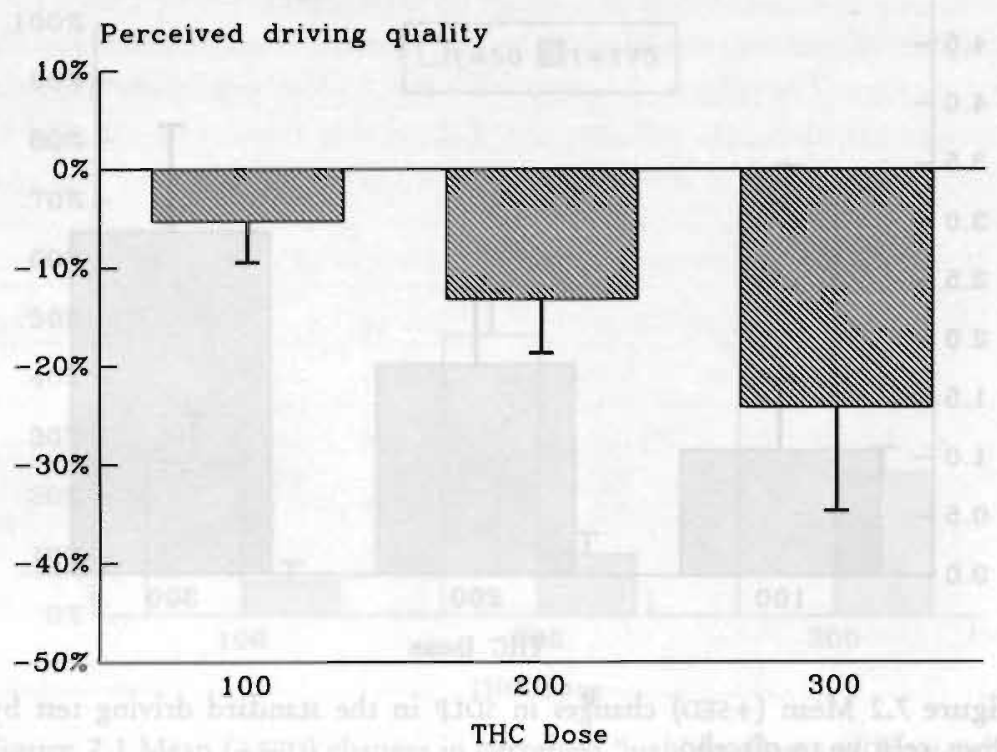

Figure 7.3 Mean (+SED) changes in perceived driving quality in the standard driving test by Drug, relative to placebo. Raw scores were expressed as percentage of 'normal'.

Changes in perceived effort ratings were trivial after the two lower THC doses. Effort did increase from $29 \%$ after placebo to $39 \%$ following the highest dose. MANOVA failed, however, to reveal any significant effects of THC on perceived effort.

Half of the subjects performed the driving tests in darkness, the other half under daylight conditions. Therefore, repeated measures analysis was again applied to the data, but now with Light/Darkness as a between-groups factor. No significant differences were found between the groups for any variable.

Driving Performance in the Car Following Test

All subjects were capable of performing the car following test in each treatment condition. There was no need for the driving instructors to ever intervene or prematurely terminate the test. In one instance, a subject needed the driving instructor's help after smoking the highest THC dose. The subject appeared confused when he took his seat in the car prior to the first car following test. The driving instructor noticed the subject's uncertainty as to what he should do 
and advised him to turn the engine on. Thereafter, the subject had no problems following the instructions and completing the ride.

Mean headway varied between conditions from 44.5 to $54.9 \mathrm{~m}$ (146 to $180 \mathrm{ft}$ ); changes relative to placebo are shown in Figure 7.4. It is interesting to note the inverse relationship between THC dose and headway: the lower the dose the greater the headway, especially in the first test after smoking. MANOVA showed that mean headway was significantly increased after smoking the lowest THC dose, relative to placebo $\left(\mathrm{F}_{1,13}=7.57 ; p<.016 ; p_{c}=.017\right)$; the two higher doses had no significant effect on headway. No significant Time or Drug by Time effects were found in either THC condition.

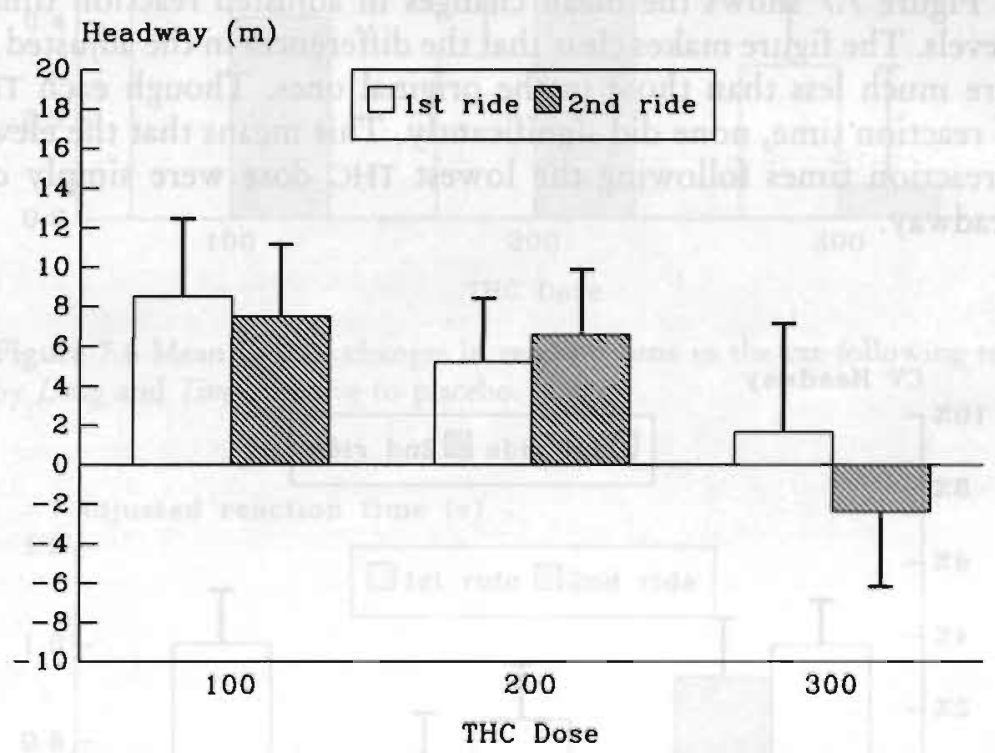

Figure 7.4 Mean (+SED) changes in headway in the car following test by Drug and Time, relative to placebo.

Coefficients of variation of headway, expressed as a percentage, were high and varied between conditions from $18.8 \%$ to $23.7 \%$. Figure 7.5 shows an inverse relationship between CV headway and administered dose, with the exception of the first ride after the highest dose. MANOVA revealed a significant effect following the lowest dose $\left(\mathrm{F}_{1,13}=16.62 ; p<.001 ; p_{\varepsilon}=.017\right)$, but not the higher doses. Drug by Time interaction approached significance following the highest dose $\left(F_{1,13}=4.85 ; p<.046 ; p_{c}=.017\right) ; 300 \mu \mathrm{g} / \mathrm{kg}$ THC produced an increased headway variability in the first test after smoking, but not in the second.

Mean reaction time to perceived changes in the preceding car's speed varied from 1.56 to $2.30 \mathrm{~s}$, between conditions. The changes, shown in Figure 7.6, 
followed a similar pattern as those of headway; the greatest increase of reaction time occurred after the lowest THC dose, and the smallest after the highest dose. Reaction times were significantly increased after smoking both $100 \mu \mathrm{g} / \mathrm{kg}$ and $200 \mu \mathrm{g} / \mathrm{kg}$ THC, but not $300 \mu \mathrm{g} / \mathrm{kg} \quad\left(\mathrm{F}_{1,13}=10.78,6.26 \& 1.88\right.$, respectively; $\left.p<.006, .027 \& .193 ; p_{c}=.017, .025 \& .05\right)$. Reaction times were, however, strongly related to mean distance ( $r=0.76$, across all conditions). This is understandable: the further apart two cars are, the more difficult it becomes for the subject to perceive changes in the preceding vehicle's velocity.

One may question what the effect of THC on reaction time would have been if subjects had driven at the same mean headway in all conditions? Covariance analysis of reaction time with headway as covariate was applied to answer this question. Figure 7.7 shows the mean changes in adjusted reaction times, from placebo levels. The figure makes clear that the differences in the adjusted reaction times were much less than those in the original ones. Though each THC dose increased reaction time, none did significantly. This means that the elevation in the raw reaction times following the lowest THC dose were simply due to a longer headway.

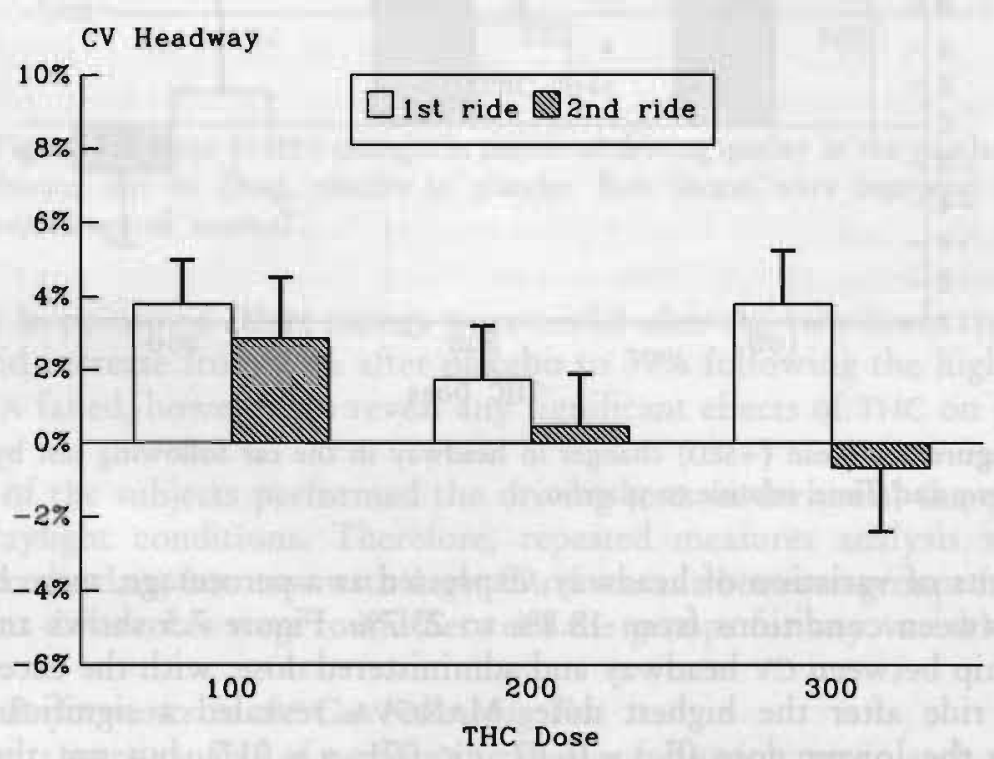

Figure 7.5 Mean (+SED) changes in CV headway in the car following test by Drug and Time, relative to placebo. 


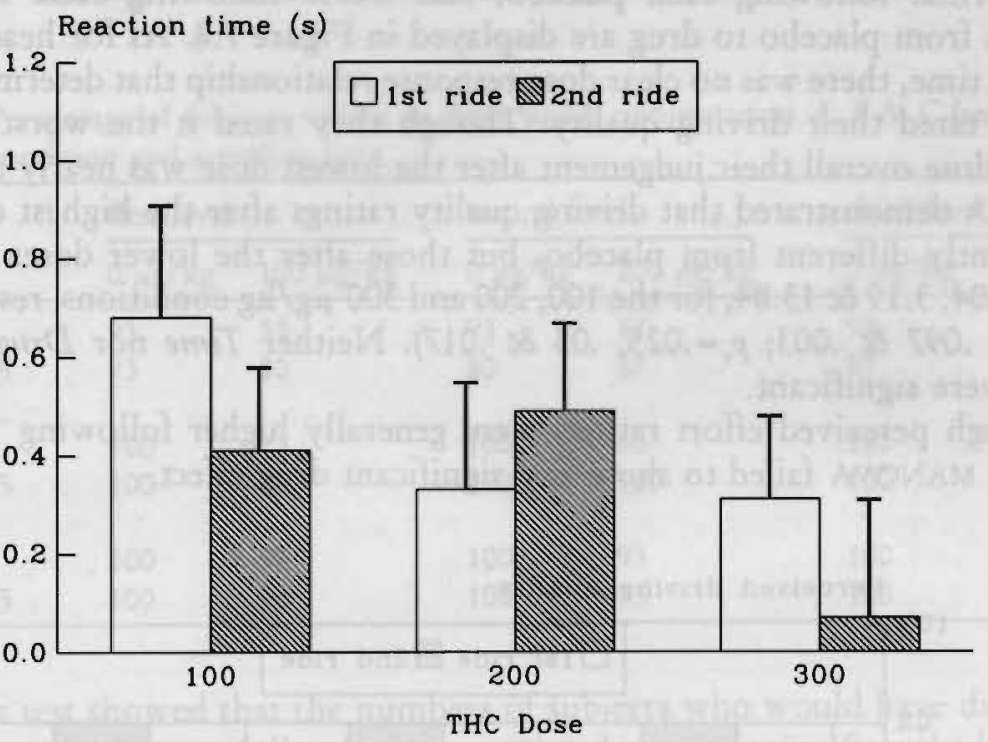

Figure 7.6 Mean (+SED) changes in reaction time in the car following test by Drug and Time, relative to placebo.

Adjusted reaction time (s)

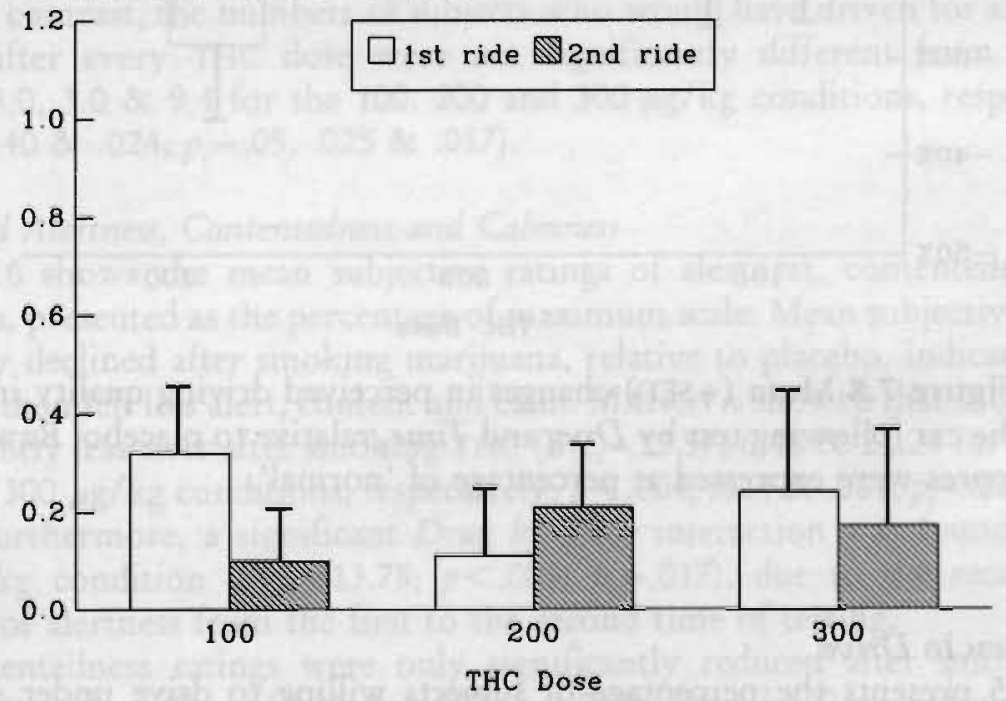

Figure 7.7 Mean (+SED) changes in reaction time, adjusted for changes in headway, in the car following test by Drug and Time, relative to placebo. 
Subjects rated their driving quality in the car following test as somewhat better than normal following each placebo, and worse following each THC dose. Changes from placebo to drug are displayed in Figure 7.8. As for headway and reaction time, there was no clear dose-response relationship that determined how subjects rated their driving quality. Though they rated it the worst after the highest dose overall their judgement after the lowest dose was nearly as critical. MANOVA demonstrated that driving quality ratings after the highest dose were significantly different from placebo, but those after the lower doses were not $\left(F_{1,13}=6.04,3.19 \& 13.84\right.$, for the 100,200 and $300 \mu \mathrm{g} / \mathrm{kg}$ conditions, respectively; $\left.p<.029, .097 \& .003 ; p_{c}=.025, .05 \& .017\right)$. Neither Time nor Drug by Time effects were significant.

Though perceived effort ratings were generally higher following THC than placebo, MANOVA failed to show any significant drug effect.

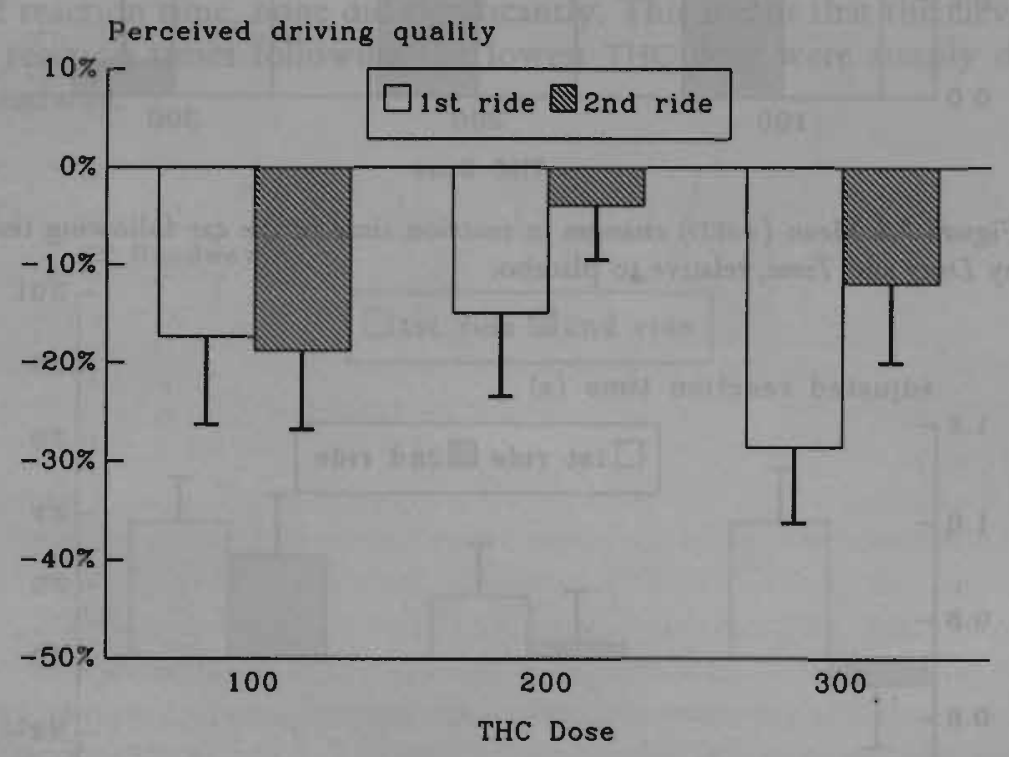

Figure 7.8 Mean (+SED) changes in perceived driving quality in the car following test by Drug and Time, relative to placebo. Raw scores were expressed as percentage of 'normal'.

\section{Willingness to Drive}

Table 7.5 presents the percentage of subjects willing to drive under specified conditions of different urgencies ( $A$. unimportant though gratifying; $B$. important but avoidable; and $C$. urgent). Subjects' responses were similar to those in the 
previous study. The lower the administered THC dose and the more urgent the reason for driving, the more subjects declared that they would be willing to drive.

Table 7.5 Percentage of subjects willing to drive under circumstances $A, B \& C$ (see text) by treatment condition and sampling time.

\begin{tabular}{|c|c|c|c|c|c|c|c|}
\hline & & first leve & & second 1 & & third le & \\
\hline & & $0 \mu \mathrm{g} / \mathrm{kg}$ & $100 \mu \mathrm{g} / \mathrm{kg}$ & $0 \mu \mathrm{g} / \mathrm{kg}$ & $200 \mu \mathrm{g} / \mathrm{kg}$ & $0 \mu \mathrm{g} / \mathrm{kg}$ & $300 \mu \mathrm{g} / \mathrm{kg}$ \\
\hline A & $t=50$ & 93 & 53 & 93 & 47 & 93 & 27 \\
\hline & $t=175$ & 93 & 80 & 80 & 87 & 93 & 53 \\
\hline B & $t=50$ & 100 & 73 & 100 & 60 & 100 & 40 \\
\hline & $t=175$ & 100 & 80 & 100 & 100 & 100 & 67 \\
\hline C & $t=50$ & 100 & 93 & 100 & 93 & 100 & 73 \\
\hline & $t=175$ & 100 & 93 & 100 & 93 & 100 & 87 \\
\hline
\end{tabular}

Cochran's test showed that the numbers of subjects who would have driven for an unimportant reason following every THC dose were significantly less than after placebo $\left(Q_{\mathrm{df}-3}=12.0,13.4 \& 21.6\right.$ for the 100,200 and $300 \mu \mathrm{g} / \mathrm{kg}$ conditions, respectively; $\left.p<.008, .004 \& .0001 ; p_{c}=.05, .025 \& .017\right)$. The same held true for the somewhat more important reason $\left(Q_{\mathrm{dr}-3}=10.2,18.0 \& 21.4\right.$ for the 100,200 and $300 \mu \mathrm{g} / \mathrm{kg}$ conditions, respectively; $p<.02, .0004 \& .0001 ; p_{c}=.05, .025 \&$ $.017)$. In contrast, the numbers of subjects who would have driven for an urgent reason after every THC dose were not significantly different from placebo $\left(Q_{\mathrm{df}-3}=3.0,3.0 \& 9.4\right.$ for the 100,200 and $300 \mu \mathrm{g} / \mathrm{kg}$ conditions, respectively; $\left.p<.40, .40 \& .024 ; p_{c}=.05, .025 \& .017\right)$.

\section{Perceived Alertness, Contentedness and Calmness}

Table 7.6 shows the mean subjective ratings of alertness, contentedness and calmness, presented as the percentage of maximum scale. Mean subjective ratings generally declined after smoking marijuana, relative to placebo, indicating that subjects then felt less alert, content and calm. MANOVA showed that subjects felt significantly less alert after smoking THC $\left(F_{1,13}=12.57,6.48 \& 21.24\right.$ for the 100 , 200 and $300 \mu \mathrm{g} / \mathrm{kg}$ conditions, respectively; $p<.004, .024 \& .001 ; p_{c}=.025, .05 \&$ .017). Furthermore, a significant Drug by Time interaction was found for the $100 \mu \mathrm{g} / \mathrm{kg}$ condition $\left(F_{1,13}=13.78 ; p<.003 ; p_{c}=.017\right)$, due to the recovery in feelings of alertness from the first to the second time of testing.

Contentedness ratings were only significantly reduced after smoking the highest THC dose $\left(\mathrm{F}_{1,13}=10.20 ; p<.007 ; p_{c}=.017\right)$. Drug by Time interaction was significant in the $100 \mu \mathrm{g} / \mathrm{kg}$ condition $\left(\mathrm{F}_{1,13}=7.71 ; p<.016 ; p_{c}=.017\right)$. Calmness ratings also declined after the higher THC doses $\left(F_{1,13}=6.05 \& 14.65\right.$ for the 200 
and $300 \mu \mathrm{g} / \mathrm{kg}$ conditions, respectively; $\left.p<.029 \& .002 ; p_{\mathrm{c}}=.025 \& \quad .017\right)$, and Drug by Time interaction approached significance in all conditions $\left(\mathrm{F}_{1,13}=4.33\right.$, $6.62 \& 5.10$ for the 100,200 and $300 \mu \mathrm{g} / \mathrm{kg}$ conditions, respectively; $p<.058, .023$ $\& .042 ; p_{c}=.05, .017 \&$ \& 025$)$.

Table 7.6 Mean (SE) ratings of alertness, contentedness, and calmness by treatment condition and sampling time.

\begin{tabular}{|c|c|c|c|c|c|c|c|}
\hline & & \multicolumn{2}{|l|}{ first level } & \multicolumn{2}{|c|}{ second level } & \multicolumn{2}{|c|}{ third level } \\
\hline & & $0 \mu \mathrm{g} / \mathrm{kg}$ & $100 \mu \mathrm{g} / \mathrm{kg}$ & $0 \mu \mathrm{g} / \mathrm{kg}$ & $200 \mu \mathrm{g} / \mathrm{kg}$ & $0 \mu \mathrm{g} / \mathrm{kg}$ & $300 \mu \mathrm{g} / \mathrm{kg}$ \\
\hline \multirow[t]{2}{*}{ alert } & $t=50$ & $78.3(3.7)$ & $59.0(4.3)$ & $76.0(4.4)$ & $63.9(4.1)$ & $80.0(3.4)$ & $57.7(4.6)$ \\
\hline & $t=175$ & $69.6(5.0)$ & $64.7(4.9)$ & $72.4(5.5)$ & $66.1(3.6)$ & $70.6(4.3)$ & $56.9(6.2)$ \\
\hline \multirow[t]{2}{*}{ content } & $t=50$ & $79.3(2.7)$ & $66.2(5.1)$ & $77.6(3.3)$ & $73.6(3.6)$ & $78.3(3.4)$ & $67.2(4.4)$ \\
\hline & $t=175$ & $72.4(3.4)$ & $70.3(4.3)$ & $74.9(4.4)$ & $73.1(3.5)$ & $76.7(3.2)$ & $65.7(4.9)$ \\
\hline \multirow[t]{2}{*}{ calm } & $t=50$ & $78.2(4.2)$ & $64.2(5.2)$ & $79.5(4.4)$ & $61.7(6.8)$ & $79.5(3.6)$ & $59.6(5.8)$ \\
\hline & $t=175$ & $72.5(5.0)$ & $72.4(3.8)$ & $75.9(4.1)$ & $71.3(4.0)$ & $77.5(3.5)$ & $70.5(4.8)$ \\
\hline
\end{tabular}

In summary, subjects felt less alert, content and calm after smoking each THC dose, relative to placebo. The effects were strongest and persisted throughout the testing session after smoking the highest dose.

\section{Critical Tracking Test}

As shown in Figure 7.9 , mean $\lambda_{c}$ measured 30 minutes after initiation of smoking diminished in a dose-related manner. Measured $31 / 4$ hours after initiation of smoking, $\lambda_{c}$ remained low after the highest, but not the two lower doses. The lowest THC dose did not affect $\lambda_{c}$ significantly. A nearly significant Drug by Time interaction was found in the $200 \mu \mathrm{g} / \mathrm{kg}$ condition $\left(\mathrm{F}_{1,13}=4.69 ; p<.05 ; p_{\mathrm{c}}=.017\right)$; i.e, that dose diminished $\lambda_{s}$ shortly after smoking, but the effect dissipated after three hours. The highest dose had a significant effect on $\lambda_{c}\left(F_{1,13}=9.03 ; p<.01\right.$; $\left.p_{c}=.017\right)$, both shortly after smoking and three hours later.

Hand Steadiness Test

Hand steadiness diminished in relation to the dose after marijuana smoking (Figure 7.10). All doses produced significantly greater instability $\left(\mathrm{F}_{1,13}=6.72,13.05\right.$ $\& 45.33$ for the 100,200 and $300 \mu \mathrm{g} / \mathrm{kg}$ conditions, respectively; $p<.022, .003 \&$. $.001 ; p_{c}=.05, .025 \& .017$ ).

Instability diminished after three hours, irrespective of the dose, resulting in significant Time effects in each drug-placebo comparison. Drug by Time interaction was not significant after the lowest dose, but approached significance after the higher doses $\left(\mathrm{F}_{1,13}=4.51 \& 6.28\right.$ for the 200 and $300 \mu \mathrm{g} / \mathrm{kg}$ conditions, respectively; $\left.p<.053 \& 026 ; p_{c}=.025 \& .017\right)$. 


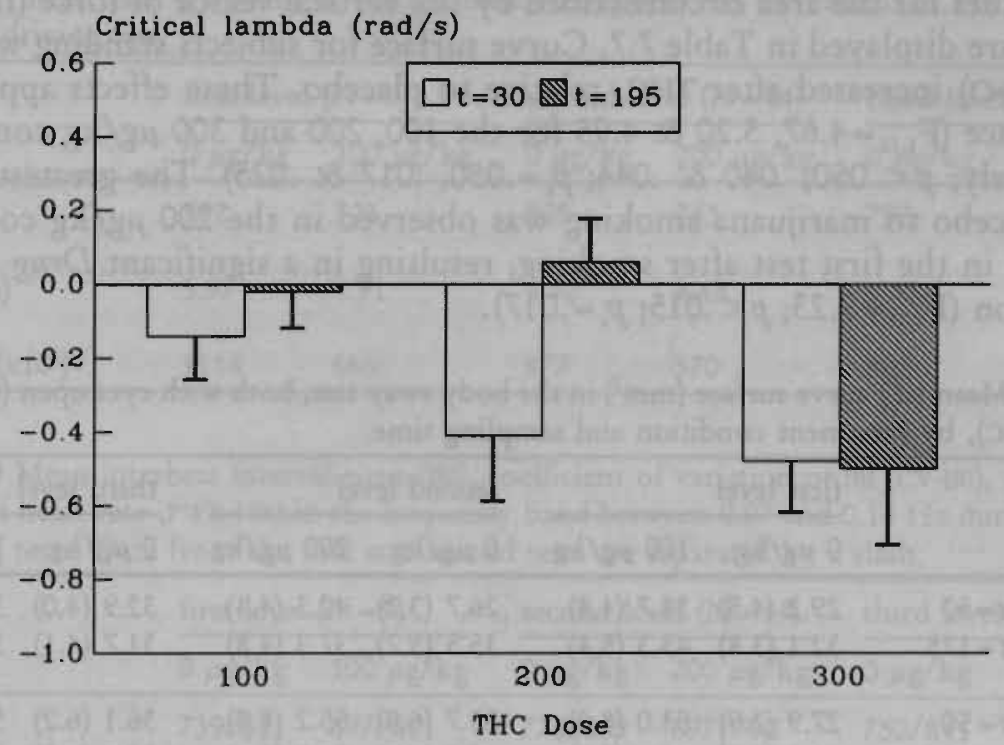

Figure 7.9 Mean (+SED) changes in $\lambda_{c}$ in the critical tracking test by Drug and Time, relative to placebo.

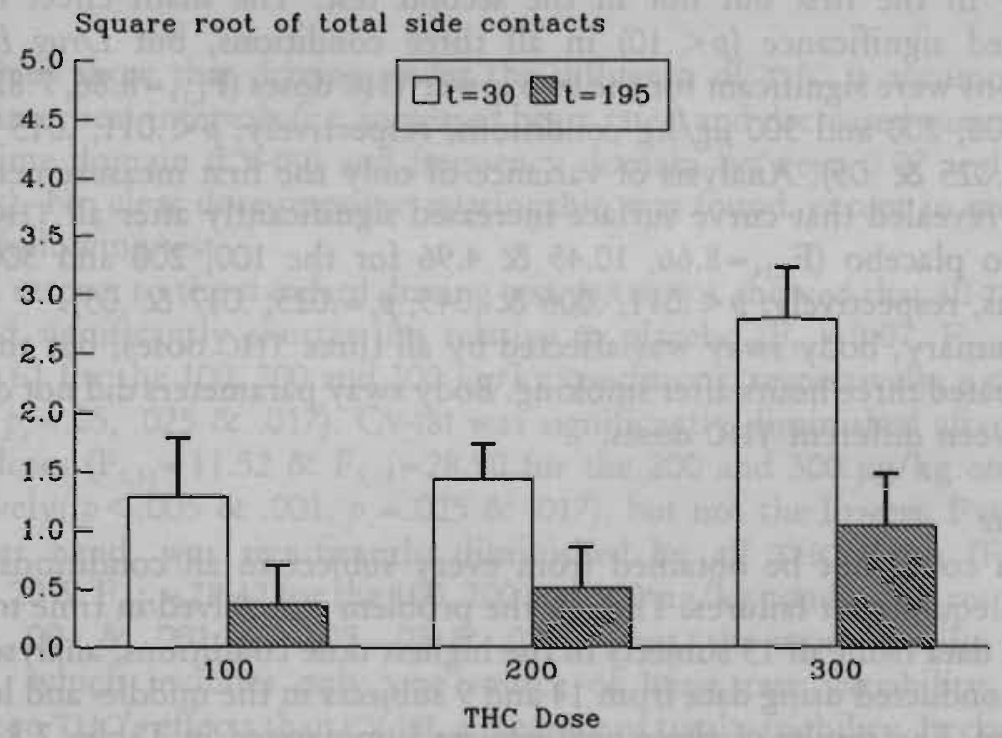

Figure 7.10 Mean $(+-S E D)$ changes in the square root of total number of side contacts in the hand steadiness test by Drug and Time, relative to placebo. 
Body Sway

Mean values for the area circumscribed by the vertical vector of force (i.e. curve surface) are displayed in Table 7.7. Curve surface for subjects standing with eyes open (CS-O) increased after THC, relative to placebo. These effects approached significance $\left(F_{1,13}=4.67,5.20 \& 4.95\right.$ for the 100,200 and $300 \mu \mathrm{g} / \mathrm{kg}$ conditions, respectively; $\left.p<.050, .040 \& .044 ; p_{c}=.050, .017 \& .025\right)$. The greatest change from placebo to marijuana smoking was observed in the $200 \mu \mathrm{g} / \mathrm{kg}$ condition, but only in the first test after smoking, resulting in a significant Drug by Time interaction $\left(\mathrm{F}_{1,13}=9.23 ; p<.015 ; p_{c}=.017\right)$.

Table 7.7 Mean (SE) curve surface $\left(\mathrm{mm}^{2}\right)$ in the body sway test, both with eyes open (CS-O) and closed (CS-C), by treatment condition and sampling time.

\begin{tabular}{|c|c|c|c|c|c|c|c|}
\hline & \multirow{2}{*}{$x^{2}+4$} & \multicolumn{2}{|l|}{ first level } & \multicolumn{2}{|c|}{ second level } & \multicolumn{2}{|l|}{ third level } \\
\hline & & $0 \mu \mathrm{g} / \mathrm{kg}$ & $100 \mu \mathrm{g} / \mathrm{kg}$ & $0 \mu \mathrm{g} / \mathrm{kg}$ & $200 \mu \mathrm{g} / \mathrm{kg}$ & $0 \mu \mathrm{g} / \mathrm{kg}$ & $300 \mu \mathrm{g} / \mathrm{kg}$ \\
\hline \multirow[t]{2}{*}{ CS-O } & $t=50$ & $29.2(4.5)$ & $38.7(4.8)$ & $26.7(3.2)$ & $42.3(4.4)$ & $32.9(4.0)$ & $39.1(4.3)$ \\
\hline & $t=175$ & $32.1(3.8)$ & $43.3(5.4)$ & $35.5(5.7)$ & $37.3(4.8)$ & $31.7(4.1)$ & $38.5(3.5)$ \\
\hline \multirow[t]{2}{*}{ CS-C } & $t=50$ & $27.9(3.9)$ & $53.0(8.6)$ & $33.7(6.8)$ & $55.2(8.8)$ & $36.1(6.2)$ & $57.7(11.2)$ \\
\hline & $t=175$ & $43.3(6.5)$ & $43.9(8.3)$ & $40.1(7.7)$ & $35.2(5.4)$ & $32.0(5.5)$ & $36.3(5.6)$ \\
\hline
\end{tabular}

Mean curve surface for the subjects standing with eyes closed (CS-C), increased markedly in the first. but not in the second test. The main effect of Drug approached significance $(p<.10)$ in all three conditions, but Drug by Time interactions were significant for the two lower THC doses $\left(F_{1,13}=8.86,7.82 \& 3.55\right.$ for the 100,200 and $300 \mu \mathrm{g} / \mathrm{kg}$ conditions, respectively; $p<.011, .015 \& .082$; $\left.p_{c}=.017, .025 \& .05\right)$. Analysis of variance of only the first measurements after smoking revealed that curve surface increased significantly after all THC doses, relative to placebo $\left(F_{1,14}=8.66,10.45 \& 4.96\right.$ for the 100,200 and $300 \mu \mathrm{g} / \mathrm{kg}$ conditions, respectively; $\left.p<.011, .006 \& .043 ; p_{c}=.025, .017 \& 2.05\right)$.

In summary, body sway was affected by all three THC doses, but this effect had dissipated three hours after smoking. Body sway parameters did not discriminate between different THC doses.

\section{Heart Rate}

ECG data could not be obtained from every subject in all conditions due to recurrent equipment failures. Though the problem was solved in time to obtain complete data from all 15 subjects in the highest dose conditions, analyses could only be conducted using data from 14 and 9 subjects in the middle- and low-dose conditions. The results of those analyses are summarized in Tables 7.8 and 7.9 for the standard and car following tests, respectively. 
Table 7.8 Mean interbeat: interval time (IBI), coefficient of variation of IBI (CV-IBI), and power density of heart rate (PWR-HR) in the frequency band between 0.07 and $0.14 \mathrm{~Hz}$ during the standard driving test.

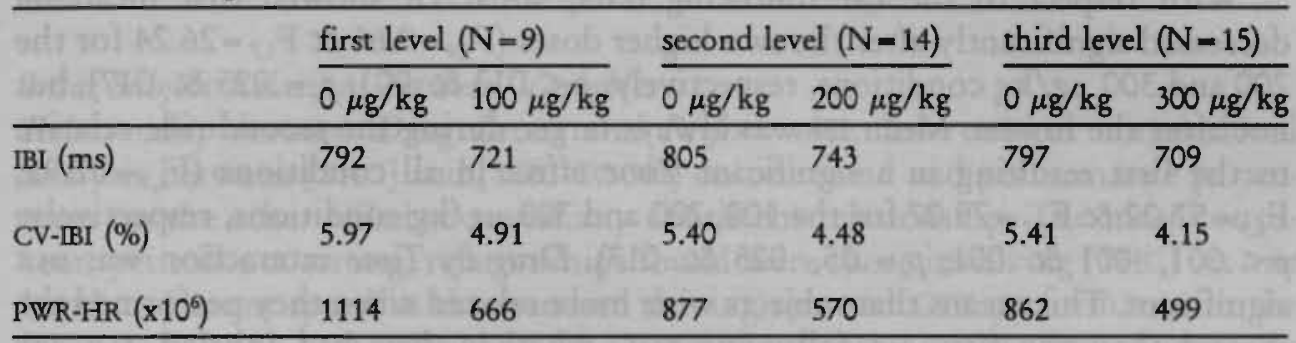

Table 7.9 Mean interbeat interval time (IBI), coefficient of variation of IBI (CV-IBI), and power density of heart rate (PWR-HR) in the frequency band between 0.07 and $0.14 \mathrm{~Hz}$ during the car following tests. Data from the first and second tests are separated by a slash.

\begin{tabular}{|c|c|c|c|c|c|c|}
\hline \multirow{2}{*}{ 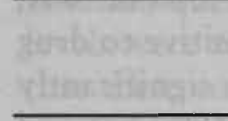 } & \multicolumn{2}{|c|}{ first level $(\mathrm{N}=9)$} & \multicolumn{2}{|c|}{ second level $(\mathrm{N}=14)$} & \multicolumn{2}{|c|}{ third level $(N=15)$} \\
\hline & $0 \mu \mathrm{g} / \mathrm{kg}$ & $100 \mu \mathrm{g} / \mathrm{kg}$ & $0 \mu \mathrm{g} / \mathrm{kg}$ & $200 \mu \mathrm{g} / \mathrm{kg}$ & $0 \mu \mathrm{g} / \mathrm{kg}$ & $300 \mu \mathrm{g} / \mathrm{kg}$ \\
\hline IBI (ms) & $739 / 821$ & $697 / 801$ & $778 / 859$ & $697 / 790$ & $750 / 841$ & $646 / 755$ \\
\hline CV-IBI (\%) & $5.48 / 6.31$ & $4.69 / 4.86$ & $5.18 / 5.83$ & $3.85 / 5.25$ & $4.67 / 5.41$ & $4.05 / 4.57$ \\
\hline PWR-HR $\left(x 10^{\circ}\right)$ & $983 / 1347$ & $608 / 807$ & $909 / 1094$ & $438 / 967$ & $726 / 993$ & $405 / 633$ \\
\hline
\end{tabular}

Both tables show that driving under the influence of THC is accompanied by shorter interbeat intervals (i.e. increased heart rates) and decreased variation both in the time domain (CV-IBI) and frequency domain between 0.07 and $0.14 \mathrm{~Hz}$ (PWR-HR). No clear dose-response relationship was found, except in mean IBI in the car following test.

With respect to the standard driving test, MANOVA showed that all THC doses produced significantly shorter IBIs relative to placebo $\left(F_{1,7}=9.07, F_{1,12}=7.63\right.$ \& $F_{t, 13}=21.61$ for the 100,200 and $300 \mu \mathrm{g} / \mathrm{kg}$ conditions, respectively; $p<.02, .017$ $\left.\& .001 ; p_{c}=.05, .025 \& 2.017\right)$. CV-IBI was significantly diminished after the two higher doses $\left(F_{1,12}=11.52 \& F_{1,13}=28.90\right.$ for the 200 and $300 \mu \mathrm{g} / \mathrm{kg}$ conditions, respectively; $\left.p<.005 \& .001 ; p_{c}=.025 \& .017\right)$, but not the lowest. PWR-HR, on the other hand, was significantly diminished by all THC doses $\left(F_{1,7}=18.62\right.$ $F_{1,12}=12.20 \& F_{1,13}=28.47$ for the 100,200 and $300 \mu \mathrm{g} / \mathrm{kg}$ conditions, respectively; $\left.p<.004, .004 \& .001 ; p_{c}=.025, .05 \& .017\right)$. Thus, the more specific measure, PWR-HR, which includes only one source of heart rate variability, is more sensitive to THC's effects than CV-IBI, a measure of total variability. In conclusion, both mean IBI and heart rate variability were significantly reduced by all THC doses. Since the latter measure is interpreted as a parameter of mental effort, it 
can be concluded that subjects needed to invest more effort in driving after smoking marijuana than after placebo.

With respect to the car following tests, MANOVA showed that mean IBI decreased significantly after the two higher doses $\left(F_{1,9}=9.66 \& F_{1,7}=26.24\right.$ for the 200 and $300 \mu \mathrm{g} / \mathrm{kg}$ conditions, respectively; $\left.p<.013 \& .001 ; p_{c}=.025 \& .017\right)$, but not after the lowest. Mean IBI was always larger during the second ride relative to the first resulting in a significant Time effect in all conditions $\left(F_{1,9}=52.92\right.$, $\mathrm{F}_{1,9}=57.02 \& \mathrm{~F}_{1,7}=79.07$ for the 100,200 and $300 \mu \mathrm{g} / \mathrm{kg}$ conditions, respectively; $\left.p<.001, .001 \& .001 ; p_{c}=.05, .025 \& .017\right)$. Drug by Time interaction was not significant. This means that subjects were more relaxed when they performed the second than the first car following test, whether they had inhaled THC or placebo.

CV-IBI was diminished after smoking THC in each condition relative to placebo and these effects were only nearly significant $\left(F_{1,9}=7.09, F_{1,9}=8.40 \& F_{1,7}=6.21\right.$ for the 100,200 and $300 \mu \mathrm{g} / \mathrm{kg}$ conditions, respectively; $p<.026, .018 \& .041$; $\left.p_{c}=.025, .017 \& .05\right)$. As in the standard test, PWR-HR was more sensitive to drug induced variability reduction; MANOVA showed that PWR-HR was significantly affected by all doses $\left(F_{1,9}=10.46, F_{1,9}=6.04 \& F_{1,7}=9.01\right.$ for the 100,200 and $300 \mu \mathrm{g} / \mathrm{kg}$ conditions, respectively; $\left.p<.010, .036 \& .020 ; p_{c}=.017, .05 \& .025\right)$. Though PWR-HR was generally higher in the second than in the first test, a significant Time effect was only found in the $200 \mu \mathrm{g} / \mathrm{kg}$ condition $\left(F_{1,9}=8.58\right.$; $\left.p<.0168 ; p_{c}=.017\right)$. Though subjects felt more relaxed in the second car following test than the first, as measured by IBI, both rides required much the same mental effort, as measured by heart rate variability.

\section{Intra-Subject Relations between Variables}

The average intra-subject correlation between [THC] and perceived 'high' was, as in the previous study, very high $(\mathrm{R}=0.90 ; p<.01)$. The correlation between measured SDLP and [THC] measured before driving was $0.63(p<.05)$, individual correlations ranging from -.14 to .96; between SDLP and [THC] measured after driving $0.57(p<.05)$, individual correlations ranging from -.10 to .92 . These correlations closely resemble those found in the previous study where SDLP correlated 0.59 and 0.42 in the first and second tests, respectively. This means that the change in driving performance, as measured by SDLP in the standard driving test, is, within individuals, moderately related to the existing [THC]. Performance in the car following test was not significantly correlated with [THC].

The square root of the number of side contacts in the hand steadiness test was significantly correlated to [THC] and perceived 'high' (both $r=.66 ; p<.01$ ). SDLP scores were significantly related to hand instability measured before driving $(r=.52 ; p<.05)$, but not with scores obtained after driving $(r=.35 ; \mathrm{ns})$. None of the other laboratory performance measures were either related to plasma concentrations of the drug or to driving performance. 
In summary, as [THC] varied within a given individual so did that individual's perceived 'high', hand steadiness and SDLP or road tracking error in the standard test.

\section{Inter-Subject. Relations between Variables}

Relationsbip between Drug Levels and Performance. Inter-subject correlational analysis between [THC] and [THC- $\mathrm{COOH}$ ] on one hand and performance parameters on the other failed to reveal any consistent relationship. The most consistent, yet still not strong, relationships found were those involving [THC] on one hand and mean speed in the standard driving test and CTT performance in the laboratory on the other (Table 7.10). Correlations with Log [THC] were generally the same as with [THC]; those with [THC-COOH] were, except a few, generally lower than those with [THC].

The correlations presented in Table 7.10 were derived from raw scores. Another question is whether changes in performance from corresponding placebo levels were related to prevailing [THC]. Correlational analyses showed, however, that these correlations were even smaller than those with the raw scores. Thus, neither [THC] nor [ $\mathrm{THC}-\mathrm{COOH}]$ predicted performance or performance impairment.

Table 7.10 Correlations between [THC] and raw performance scores in the marijuana conditions. If performance was measured only once (like SDLP), then the same scores were correlated with [THC] values as measured before and after the driving tests.

$\frac{100 \mu \mathrm{g} / \mathrm{kg}}{1^{\text {st }} \quad \frac{200 \mu \mathrm{g} / \mathrm{kg}}{2^{\text {nd }}}} \quad \frac{\frac{300 \mu \mathrm{g} / \mathrm{kg}}{1^{\text {s }}} 2^{\text {nd }}}{1^{\text {st }}}-$

\begin{tabular}{lllllll}
\hline Standard Driving Test: & & & & & & \\
SDLP & -.33 & +.02 & -.13 & +.09 & -.25 & -.18 \\
SP & -.26 & -.18 & -.50 & -.20 & $-.55^{*}$ & -.61 \\
SDSP & -.22 & -.14 & -.34 & -.32 & -.20 & -.15 \\
Car Following Test: & & & & & & \\
adjusted RT & -.18 & +.20 & +.41 & +.24 & +.04 & +.05 \\
headway & +.32 & +.15 & +.39 & -.02 & +.18 & +.05 \\
CV headway & -.21 & +.40 & +.27 & +.43 & -.29 & -.09 \\
Laboratory Tests: & & & & & & \\
$\lambda_{\text {c }}$ & $+.63^{\circ}$ & +.31 & +.48 & +.27 & +.45 & +.18 \\
side contacts & $+.45^{\circ}$ & -.38 & $+.54^{\circ}$ & +.32 & +.44 & +.50 \\
CS-O & -.25 & -.13 & +.43 & +.02 & -.38 & +.06 \\
CS-C & -.05 & -.02 & +.36 & -.25 & -.26 & $-.37^{\prime}$ \\
\hline
\end{tabular}

Relationship between Driving Impairment and' Willingness to Drive. In general, the average road tracking impairment in the standard test of those subjects, who indicated before the driving tests commenced that they would normally not have driven under the imagined circumstances, was greater than of those who would 
have driven. Statistically, these effects were neither significant when willingness to drive for 'unimportant though gratifying' reasons was used as the grouping factor, nor when willingness to drive for 'urgent' reasons was used. This was probably due to the small number of subjects who would not have driven under the former condition, and would have driven under the latter condition. When willingness to drive for 'important, but avoidable' reasons was used as the grouping factor, no significant difference in road tracking impairment was found between the groups after the highest THC dose; but, following the two lower doses, subjects who would not have driven were significantly more impaired than those who would have driven $\left(F_{1,13}=10.38 \& 6.93\right.$ for the 100 and $200 \mu \mathrm{g} / \mathrm{kg}$ conditions, respectively; $\left.p<.007 \& .021 ; p_{c}=.017 \& .025\right)$.

Relationship between Driving Performance and Other Measures. In the previous study, SDLP was moderately correlated with $\lambda_{c}$ from the CTT. The present study showed the same pattern of correlations i.e. -.41 and -.50 in the $100 \mu \mathrm{g} / \mathrm{kg}$ condition, -.49 and -.37 in the $200 \mu \mathrm{g} / \mathrm{kg}$ condition, and $-.58(p<.05)$ and -.54 $(p<.05)$ in the $300 \mu \mathrm{g} / \mathrm{kg}$ condition. Despite the interesting theoretical aspects of these consistent results, correlations of such magnitude are generally considered too low for predictive purposes. Performance in the car following test was not significantly correlated with $\lambda_{c}$. Neither hand steadiness nor body sway were related to performance in the standard driving test or the car following test.

As in the previous study, repeated measures analysis of SDLP in the standard test was repeated with reported Frequency of cannabis smoking as a betweensubjects factor. MANOVA failed to reveal any significant effect.

The relationship between test performance and previous experience of driving under the influence could not be determined: too few indicated having driven in that condition more than a few times (Table 7.1).

\subsection{Discussion}

The conservative approach of administering THC according to an ascending, placebo controlled, dose series achieved its purpose of ensuring the subjects' safety. All were able to complete the series without suffering any untoward reaction while driving. However it is fair to ask whether this approach could have conceivably biased the results toward minimization of the drug's effects. We have three reasons for believing that this was not the case. Driving performance during successive repetitions of the standard highway driving test after placebo smoking was relatively constant, so that the reference for judging effects of every dose's effects was essentially the same. The measured mean changes in SDLP after the lowest and highest THC doses in the present study were about what they had been in the previous study where dose orders were counterbalanced. Finally, 
there was apparently one dose sequence effect on car following performance but it was the opposite of any which could bias the results toward minimizing THC's effects. Such a bias would have existed if the subjects choose to operate more cautiously as doses ascended. Yet as described below, they appeared to operate most cautiously in the car following test after the lowest dose. If any bias existed in this test it was toward maximizing, rather than minimizing THC effects.

Road tracking performance in the standard test was impaired in a dose-related manner by THC. The $100 \mu \mathrm{g} / \mathrm{kg}$ dose produced a slight elevation in mean SDLP $(1.1 \mathrm{~cm})$, albeit nearly significant. The $200 \mu \mathrm{g} / \mathrm{kg}$ dose produced a significant elevation $(1.8 \mathrm{~cm})$, of dubious practical relevance. The $300 \mu \mathrm{g} / \mathrm{kg}$ dose produced a highly significant elevation $(2.9 \mathrm{~cm})$ which may be viewed as practically relevant but unexceptional in comparison with similarly measured effects of many medicinal drugs.

For example, diazepam given for one week in its lowest therapeutic dose (5 $\mathrm{mg}$, thrice daily) caused anxious patients to drive with a mean SDLP about $7 \mathrm{~cm}$ higher than their premedication baseline (Van Laar et al., 1992). Furthermore, THC's effects on SDLP were, after the 100,200 and $300 \mu \mathrm{g} / \mathrm{kg}$ doses in this study, about the same as those of BACs $=0.02,0.04$ and $0.06 \mathrm{~g} \%$ according to the empirical equation described by Louwerens et al. $(1985,1987)$. The comparison reinforces our impression of the relevance of SDLP changes after THC. The two lower doses produced elevations less than the lowest BAC associated with intoxication and an elevated risk of causing a traffic accident (i.e. $0.05 \mathrm{~g} \%$; Borkenstein et al., 1974; Council on Scientific Affairs, 1985), whereas the highest dose, and one preferred by the drug's users, produced a marginally greater elevation. But even this change in SDLP can not, by itself, be taken to indicate exceptional impairment.

It is often reported that subjects compensate for THC's adverse effects on driving abilities by operating at slower than normal speeds, especially through curves or slaloms. Our subjects were instructed to maintain a speed of $95 \mathrm{~km} / \mathrm{h}$ $(58 \mathrm{mph})$ unless compelled to slow down for safety reasons. Following marijuana smoking they drove with an average speed that was only slightly lower (maximum, $1 \mathrm{~km} / \mathrm{h}$ or $0.6 \mathrm{mph}$ ) than after placebo and very close to the prescribed level. They apparently felt that it was within their capability to safely perform this relatively simple task while operating at the normal highway speed.

The objective of confirming results obtained in the previous closed highway study was achieved in this one. It should be recalled that subjects' SDLPS were measured twice on the former occasion; i.e. in $22 \mathrm{~km}(13.7 \mathrm{mi})$ trials beginning 40 and 100 minutes after the initiation of smoking. The timing of the second trial most closely corresponded to that in the present study so their respective results will serve as the basis for comparison. Mean SDLP elevations after the lowest and highest doses differed little between the two studies: $100 \mu \mathrm{g} / \mathrm{kg}, 1.4$ and $1.1 \mathrm{~cm}$; and $300 \mu \mathrm{g} / \mathrm{kg}, 2.4$ and $2.9 \mathrm{~cm}$, respectively. Mean SDLP elevations after the 
$200 \mu \mathrm{g} / \mathrm{kg}$ dose differed somewhat between studies, being $3.3 \mathrm{~cm}$ in the first case and 1.8 in the second. Yet the results of the first study were anomalous in two respects. Not only did the former group's reaction in the $2^{\text {nd }}$ trial exceed that in the $1^{\text {st }}$ trial following the $200 \mu \mathrm{g} / \mathrm{kg}$ dose, it was also greater than their $2^{\text {nd }}$ trial reaction after the $300 \mu \mathrm{g} / \mathrm{kg}$ dose. Both results were in contradiction to plasma THC concentrations measured at these times. Thus the peculiar elevation in mean SDLP during the $2^{\text {nd }}$ trial after the intermediate dose was probably a consequence of sampling error. That it was not replicated in the present study should dispel any notions to the contrary.

The car following test in its present form was implemented for the first time in the present study. In it, subjects maintained a headway of $45-50 \mathrm{~m}(148-164 \mathrm{ft})$ while driving in the successive placebo conditions. They lengthened mean headway by 8,6 and $2 \mathrm{~m}(26.2,19.7$ and $6.6 \mathrm{ft})$ in the corresponding THC conditions after 100,200 and $300 \mu \mathrm{g} / \mathrm{kg}$, respectively. The initially large drugplacebo difference and its subsequent decline is a surprising result in need of an explanation. If one considers that changes in headway after THC results from impaired distance perception, the inverse relationship between administered dose and mean headway defies explanation. But if one considers these differences as the results of the subjects' caution in approaching the task under the influence of THC on successive occasions, another explanation seems plausible.

Performing the car following test in the company of investigators while under the influence of THC was a novel experience for all subjects. Neither the investigators nor the subjects could predict how the latter would be able to operate the vehicle. The former were somewhat apprehensive and it would not be surprising if the latter were also, particularly, on the first occasion the test was performed after THC inhalation. Subjects were required to match their vehicle's speed with that of the preceding vehicle's and so were unable to reduce velocity as a compensatory action. They could however maintain a longer headway and thereby slightly increase their margin of safety which might be needed if THC retarded their reactions. We believe they did so after receiving the lowest THC dose. When nothing untoward happened and the subjects' confidence in their ability to control the vehicle grew, they apparently tended to diminish headway after each of the succeeding doses so that it came progressively closer to the distance measured after all of the placebo treatments. Thus our explanation for the initial difference between headways maintained after THC and placebo, and why it diminished in subsequent pairs of these conditions, is that the subjects' caution was greatest the first time they undertook the test under the influence of THC and progressively less thereafter.

The only other plausible explanation is that lower THC doses induce a greater sense of caution than higher doses. There is of course no way to determine which of the two explanations is valid from the results of this study. But the fact that the subjects reported feeling less calm and content as the doses increased 
seems to contradict the notion that they simultaneously became less cautious. Whatever explanation is favored, it is clear that large doses of THC have little effect on mean headway during car following.

Reaction time to changes in the preceding vehicle's speed increased following THC treatment, relative to placebo. The administered THC dose was inversely related to the change in reaction time, as it was to headway. Mean increases in reaction time were $0.55,0.41$ and $0.19 \mathrm{~s}$ following the 100,200 and $300 \mu \mathrm{g} / \mathrm{kg}$ dose, respectively. However, reaction time data were confounded with headway. That is, increased reaction times were partly due to longer headway. Statistical adjustment for this confounding resulted in smaller and non-significant increases in reaction time following marijuana treatment, the greatest impairment $(0.32 \mathrm{~s})$ being observed in the first test following the lowest THC dose. Headway variability followed a similar pattern as mean headway and reaction time; the greatest impairment was found following the lowest dose.

A secondary objective of the study was to determine whether degrees of impairment would correlate between the two tests in a manner indicating a general influence of THC on driving behavior. The results obtained in this study showed that this was not the case; a significant dose-related impairment was found with road tracking, but not with car following. Test duration can not have been the critical point, since the standard test in the previous study was of the same duration as the present car following test.

The car following test was both less sensitive and reliable than it could be following the removal of certain procedural flaws. Intervals between successive maneuvers were practically constant and the preceding vehicle's deceleration was both abrupt an stereotyped. Thus the occurrence and nature of the maneuvers were highly predictable for the subjects.

Greater irregularity in both the timing of maneuvers and the profile of velocity changes would probably increase the sensitivity of the test. After the fact we recognized that the indirect and discontinuous method used for measuring headway produced a degree of error variance which appreciably reduced the reliability of these data. Equipment has become available since the initiation of this study which now makes it possible to measure headway directly and continuously (i.e. an inexpensive pulsed laser reflection recording system). Use of this equipment should increase the reliability of headway measurement in future applications of this test. Finally, the greatest source of error variance was the procedure of allowing the subject to assume, within limits, whatever headway he/she chooses. Whether this is a flaw or not depends upon one's desire to measure or control headway at the beginning of maneuvers. However the subject's choice of headway is certainly a factor which inflates reaction time error variance. Adjusting the data statistically to overcome the confounding effect of headway on reaction time is only a partial solution to the problem. We would 
probably have been wiser to strictly enforce headway control in order to increase the reliability of reaction time measurements and their sensitivity to drug effects.

Without trying to minimize the impact of procedural errors on the data, it is doubtful whether any flaw or all in combination seriously obscured a practically important THC effect. SDLP recorded in the standard highway driving test was about as sensitive to low-dose THC effects as any of the traditional laboratory performance measures taken in the study. Moreover the average intra-subject correlation between [THC] and SDLP was as high or higher than any measured between the drug's plasma concentration and another performance variable. This relationship could not have been measured if SDLP were not one of the most sensitive parameters known for measuring the effects of THC. The fact that car following performance was less sensitive does not mean that it would have failed to reveal effects of practical importance if these truly existed. SDLP showed no such effects even after the highest dose.

The reasons why SDLP and not car following performance showed the modest impairing effects of THC may have less to do with the inadequacy of the latter test than to the difference between what the two tests measure. SDLP is controlled by a very fast and high capacity human information processing system which operates in a wholly 'automatic' manner. That is, outside of conscious control. The process is relatively impervious to environmental changes as shown again by the high reliability of SDLP under repeated placebo conditions in the present study. It is, however, highly vulnerable to internal factors that retard the flow of information through the system. THC and other drugs are among these factors. When they interfere with the process to elevate SDLP there is very little the afflicted individual can do in way of compensation. Car following performance on the other hand depends upon more discrete perception of events leading to a conscious decision, a response selection and its execution. Performing the test involves far more sustained attention and conscious effort than does road tracking in the standard test. Because car following performance is under conscious control and well within the speed limitations of 'cognitive' compensatory mechanisms it is possible for individuals to recognize their deficiencies and correct them by effort that increases attention. In short, any deficiencies that THC might have otherwise produced may have been overcome by the subjects' compensatory effort. The cost of effort focused on accomplishing a task is however accompanied by less capacity left for performing another in parallel. The subjects indeed related that their investment of effort in the first car following test increased with the administered dose, and relative to corresponding placebo levels, more so than in the standard driving test. Though these differences were not statistically significant in either case, they were in line with the significant reduction in heart rate variability, that occurred independently of mean rate changes after every THC dose, including the lowest. Together the findings support the premise that THC increases the requirement for compen- 
satory effort during car following which maintains constant performance, but possibly reduces the capacity for undertaking any activity in parallel. Coupled with THC's reputably adverse effect on the ability to divide attention between tasks performed simultaneously (Smiley, 1986), the net effect might constitute a more serious impediment to safe driving than any observed in this study. It will be interesting to explore this possibility in further research.

Subjects' report of their willingness to drive under specified conditions of different urgencies were similar to those in the previous study. The lower the administered THC dose, and the more urgent the reason for driving, the more subjects declared that they would be willing to drive. Furthermore, there was a tendency for subjects who would normally not have driven to be more impaired in road tracking than those who would. Apparently these subjects recognized their respective degrees of impairment while under the influence of every THC dose. This is supported by the subjects' judgments of their own driving quality which changed in a realistic dose related manner after marijuana smoking.

Critical tracking and hand steadiness tests were more sensitive to THC induced impairment than in the previous study. This confirms our impression that the earlier CTT version was poorly conceived. It also indicates that applying the correct procedures for measuring hand steadiness is very important; i.e. subjects should not be allowed to rest their hand on the table while performing the test. Both tests showed dose-related impairment shortly after cessation of smoking. When the tests were repeated three hours later, hand instability was still present though to a lesser degree. The effect of the highest but not the two lower doses on CTT performance persisted undiminished from the first to the second test, or over a 3-hour period after smoking. These results partially confirm results obtained by Sharma and Moskowitz (1975). They found that THC $200 \mu \mathrm{g} / \mathrm{kg}$ had a virtually undiminished effect on tracking performance for up to four hours. The time course of postural instability after marijuana smoking followed a different profile. All THC doses increased body sway to the same extent shortly after cessation of smoking but none did three hours later.

An important practical objective of this study was to determine whether degrees of driving impairment can be accurately predicted from either measured concentrations of THC in plasma or performance measured in potential roadside 'sobriety' tests of tracking ability or hand and posture stability. These results, like many reported before, indicate that none of these measures accurately predicts changes in actual driving performance under the influence of THC. CTT performance came closest but even its correlation with driving was only $r=.50$. However, that test might well be included in a battery of similarly predictive tests, measuring different abilities, to collectively yield a single more predictive index of impairment. 


\section{CHAPTER 8}

\section{MARIJUANA, AlCOHOl AND URBAN CITY DRIVING}

\subsection{Introduction}

In the previous study, THC doses of $200 \mu \mathrm{g} / \mathrm{kg}$, and higher, produced significant impairment of road tracking, but not car following, performance. The lowest dose, $100 \mu \mathrm{g} / \mathrm{kg}$, failed to produce significant effects on road tracking, but increased headway during car following. The latter observation was interpreted as the result of increased caution, since mean headway did not change following subsequent higher THC doses. It therefore seems that low doses of THC do not, or only slightly, impair driving performance. Yet normal driving is far more complex and varied than simply maintaining a safe lateral position and headway during uninterrupted travel on a highway. A THC dose having no effect on these parameters might still impair driving performance in more complex urban driving situations.

There were logical and safety reasons for restricting the THC dose in the third driving study to that which had failed to produce significant impairment in the second. Both the 200 and $300 \mu \mathrm{g} / \mathrm{kg}$ doses impaired performance on the highway and could be expected to do so again in the urban driving environment. There the consequences of high dose THC effects are more difficult to predict and therefore safely control. The $100 \mu \mathrm{g} / \mathrm{kg}$ dose had some significant effects on the highway but none that could rightfully be called dangerous. This dose might still cause impairment in more complex city driving, but the risk was judged to lie within the realm of the acceptable. For that reason it was given to a group of regular cannabis users, along with placebo in the present study.

For comparative purposes another group of regular alcohol users were treated with a modest dose of their preferred recreational drug, and again placebo, before undertaking the same test. It was hoped that this addition would not only verify the sensitivity of the test but also allow a comparison between effects of recreational drugs that Dutch society considers as illicit and licit when both are given in relatively low doses. The comparison was not designed to show that one drug is 'safer' for use by drivers than the other. Surely neither are safe when consumed before driving in high doses. However if respective low dose effects are 
comparable then one would be justified to conclude that THC may be considered as posing a traffic safety hazard which is in some respects similar to alcohol's.

The only study that has been conducted in actual traffic before this program started was also a city driving study. Klonoff (1974) assessed the effects of two THC doses, 4.9 and $8.4 \mathrm{mg}$ THC which are equivalent to 70 and $120 \mu \mathrm{g} / \mathrm{kg}$ for a $70 \mathrm{~kg}(154 \mathrm{lb})$ person. Aspects of subjects' driving performance were scored by a professional examiner using an abbreviated version of the British Columbia Department of Motor Vehicles' standard driver's licencing test. The results showed that subjects performed less competently when under the influence of the highest, but not the lowest, dose. In particular, they scored lower on judgment and concentration scales. Moskowitz (1985) and Smiley (1986) criticized the method of measuring driving performance on the grounds that the examiners' reliability was never determined and that the scoring instrument had never been shown to provide measures related to driving safety. Smiley questioned, for example, whether ratings of posture and irritability are relevant for good driving performance. These are sound criticisms but one has to assume that Klonoff's approach should have been sensitive to serious driving performance impairment, related to safety, if it had in fact occurred.

Two scoring methods were employed in the present study. The first was in fact a method similar to that applied by Klonoff; i.e. the driving instructor acting as the safety controller during the tests retrospectively rated the driver's performance using a standard scale. This method has been applied previously to show the impairing effects of alcohol (De Gier, 1979) and diazepam (De Gier et al., 1981) in similar situations. Jones (1978) criticized this use of driver licensing assessment procedures. She opposed the lack of precise definitions for many of the behaviors rated by examiners and the requirement for rating all of them at once. In contrast to this 'molar' approach, she developed a more 'molecular' one for evaluating driving proficiency. Her method was also applied in the present study. It involves the employment of specially trained observers who apply simple and strict criteria for recording when the driver makes or fails to make each in a series of observable responses at predetermined points along a chosen route.

The professional observer's global ratings are inherently less reliable than the scores obtained by the molecular rating scheme. Still the molar approach has some advantages. The professional's experience with many drivers operating in all traffic situations provides him with the ability to integrate far more information than is possible to obtain from limited performance sampling. He has internalized a broad concept of acceptable driving performance and applies more flexible criteria for judging when it is unsafe within a particular test situation. Of course the danger that a professional's biases may influence his judgments needs to be overcome by training and his adherence to structured rules which are specific for the investigation. But when this is done, he may provide a more valid 
estimation of the overall safety of a subject's driving performance. If this were not the case it would be difficult to explain how every developed society relies upon the professional's and not a traffic scientist's opinion of whether a particular individual should be licensed to drive.

The objective of this study would be satisfied in one way if neither observer rating method yielded a significant difference between driving performance after $100 \mu \mathrm{g} / \mathrm{kg}$ THC and placebo. These results would confirm those obtained in the previous study by indicating that the selected dose lies below that capable of impairing driving performance. This conclusion would only be warranted, however, if it could be shown that the tests were sensitive enough to measure significant driving performance impairment after alcohol relative to placebo. If that were not the case, test insensitivity could be judged as the factor responsible for negative results, rather than the lack of a THC effect.

The objective would be satisfied in another way if either or both rating methods showed significant impairment after THC. Such results would indicate that any dose likely to be consumed before driving should be considered hazardous, regardless of whether alcohol's effects were the same, more or less. In the event that significant impairment occurred after THC, we were prepared to determine its relationship with plasma concentrations of THC and $\mathrm{THC}-\mathrm{COOH}$ measured at about the same time.

\subsection{Methods}

\section{Subjects}

Two groups of sixteen new subjects apiece, equally comprised of men and women, participated in the study. The groups will be referred to by the alcohol and marijuana group. Subjects in both groups were recruited according to the same inclusion/exclusion criteria as before with one exception. Subjects in the alcohol group were regular users of alcohol but not marijuana (see below).

All subjects were individually trained to perform the city driving test in a preliminary 'dress rehearsal' and were familiarized with the other tests and questionnaires.

Plasma analyses after conclusion of the study showed that two males' plasma contained neither THC nor THC-COOH in any sample. It was concluded that these subjects had not inhaled smoke so their data were excluded from further analyses.

Characteristics of the remaining subjects are given in Table 8.1. Except for the reported incidence of marijuana use, there were no significant differences between any of the groups' characteristics. 
Table 8.1 Mean \pm SD (range) of subjects' characteristics.

\begin{tabular}{lll}
\hline & alcohol group & marijuana group \\
\hline $\mathrm{N}$ & 16 & 14 \\
age (yrs) & $23.7 \pm 2.7(20-28)$ & $22.4 \pm 3.5(20-34)$ \\
weight (kg) & $68.1 \pm 7.9(60.0-86.1)$ & $67.9 \pm 8.9(54.0-90.5)$ \\
weight (lb) & $150.1 \pm 17.4(132.2-189.8)$ & $149.7 \pm 19.6(119.0-199.5)$ \\
\# alcoholic drinks/week & $8.3 \pm 6.2(1-21)$ & $10.6 \pm 8.4(1-30)$ \\
smoking experience (yrs) & 0 & $3.1 \pm 2.1(1-10)$ \\
$\#$ \# joints/month & 0 & $2.3 \pm 2.5(1-8)$ \\
driving experience (yrs) & $4.8 \pm 2.6(2-10)$ & $4.1 \pm 3.3(2-15)$ \\
driving experience (km x 1000) & $35.3 \pm 28.3(5-90)$ & $28.6 \pm 45.7(6-180)$ \\
driving experience (mi x 1000) & $21.9 \pm 17.6(3-56)$ & $17.8 \pm 28.4(4-112)$ \\
\hline
\end{tabular}

\section{Design, Doses and Administration}

The study was conducted according to a mixed between-groups within-subjects design, one group drinking alcohol and placebo alcohol and the other smoking marijuana and placebo marijuana. Alcohol was administered as $99.8 \%$ ethanol mixed with orange juice and Grand Marnier essence to a volume of $250 \mathrm{ml}$. The dose was $0.43 \mathrm{~g} / \mathrm{kg}$ lean body mass (on average, this resulted in a dose of $0.36 \mathrm{~g} / \mathrm{kg}$ body weight in males, and $0.31 \mathrm{~g} / \mathrm{kg}$ in females). Lean body mass was calculated by subtracting the percentage of fat, determined by skinfold thickness (Durnin and Womersley, 1974), from total body weight. The dose was chosen to yield a Blood Alcohol Concentration (BAC) approaching $0.05 \mathrm{~g} \%$ when the driving test commenced 45 minutes after onset of drinking. Subjects were instructed to fast $2 \frac{1}{2}$ hours before drinking and to ingest the dose within 5 minutes. Alcohol in the subjects' expired air was monitored using a Lion S-D3 Breath-Alcohol Analyzer to ensure that subjects would not drive with BACs that were higher than $0.05 \mathrm{~g} \%$, which is the legal limit in The Netherlands.

The subjects in the marijuana group were treated on separate occasions with THC doses of $\mathrm{O}$ (placebo) and $100 \mu \mathrm{g} / \mathrm{kg}$. Marijuana cigarettes were prepared from batches containing $1.77 \%$ THC. Cigarettes appeared identical in both treatment conditions and were smoked through a plastic holder in a fashion determined by the subject but with the constraint that smoking had to be finished within five minutes. After cessation of smoking, cigarettes were retained for subsequent gravimetric estimation of THC consumed. These analyses revealed that the average $( \pm S D)$ amount of consumed THC in the marijuana condition was 6.9 $( \pm 1.0) \mathrm{mg}$ or $101( \pm 6) \mu \mathrm{g} / \mathrm{kg}$.

Half of both groups received the treatments in the same order, the others, in reverse order. The driving instructor and observer who rated subjects' driving performance were blind regarding both the administered drug (active or placebo) and the subject's group membership. 


\section{Testing Procedures}

Upon arrival at the laboratory, subjects were tested for the presence of alcohol in breath. Subjects in the marijuana group were further tested for the presence of cannabinoids and other drugs in urine (as described in Chapter 4). If cannabinoids were found, a blood sample was taken for later verification of the presence of THC. The schedule of further activities on test-days is shown in Table 8.2.

Table 8.2 Schedule of activities on test-days.

\begin{tabular}{lll}
\hline & relative time (min) & \\
\cline { 2 - 3 } activity & alcohol group & marijuana group \\
\hline drinking / smoking & $0-5$ & $0-5$ \\
hand steadiness and time perception tests & $30-35$ & $15-20$ \\
blood sampling and questionnaires & $35-40$ & $20-25$ \\
city driving test & $45-95$ & $30-80$ \\
blood sampling and questionnaires & $95-100$ & $80-85$ \\
hand steadiness and time perception tests & $105-110$ & $90-95$ \\
\hline
\end{tabular}

\section{Driving Test and Scoring Methods}

Driving tests were conducted in daylight over a constant $17.5 \mathrm{~km}(10.9 \mathrm{mi})$ route within the city limits of Maastricht (population ca. 115,000). The route was constructed through business and residential areas on 2-lane undivided streets and included a $6 \mathrm{~km}$ ( $3.7 \mathrm{mi})$ 4-lane divided segment on a major cross-city thoroughfare (Figure 8.1). Subjects drove their placebo and active drug rides through heavy, medium and low density traffic on the same day of the week, and at the same time of day. Maneuvers included left and right turns at some intersections and driving through others, left and right lane changes, and responding to traffic control devices (i.e. stop signs and signals). There was one special maneuver, repeated twice; i.e. executing a Y-turn on a residential street. A schematic representation of the standard route is shown in Figure 8.1 and the symbols used for maneuvers and road densities are shown in Table 8.3 (the performance variables will be discussed below).

Driving tests were conducted in a dual control 2-door sedan (Volkswagen Golf) normally used for driving instruction/examination. Two persons accompanied the subject: a licensed driving instructor sitting in the front passenger's seat and an trained observer sitting in the center of the rear seat. The former had access to redundant controls and his primary responsibility were controlling safety and giving the route instructions; and, to rate the driver's performance retrospectively (below) after the ride. The observer in the rear seat scored the driver's performance 'on-line' and timed the duration of the ride. 


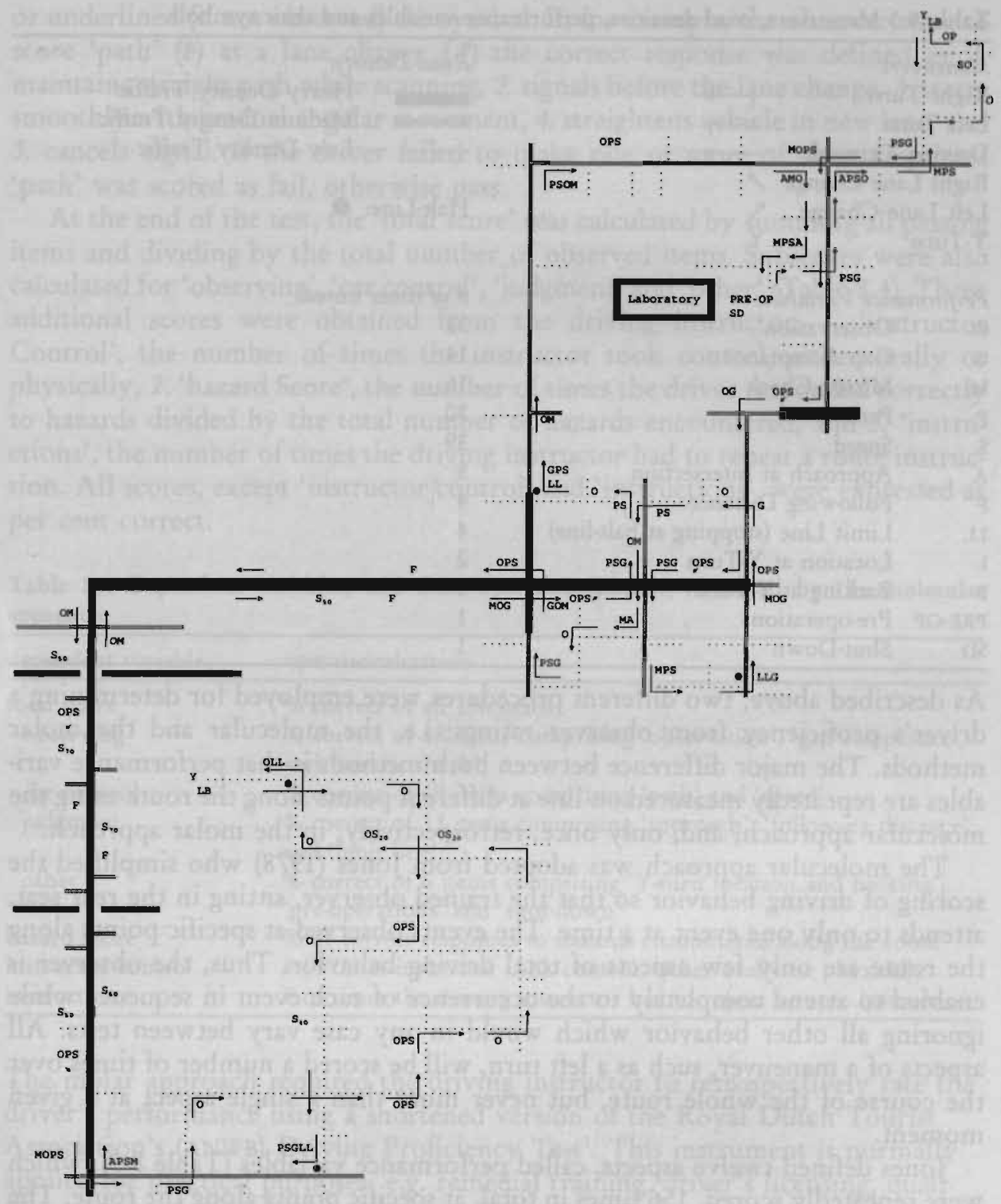

Figure 8.1 Scoring sheet for molecular approach, consisting of the schematic route map with symbols indicating the driver behavior to be scored at each point (for explanation of the symbols, see Table 8.3). 
Table 8.3 Maneuvers, road densities, performance variables and their symbols.

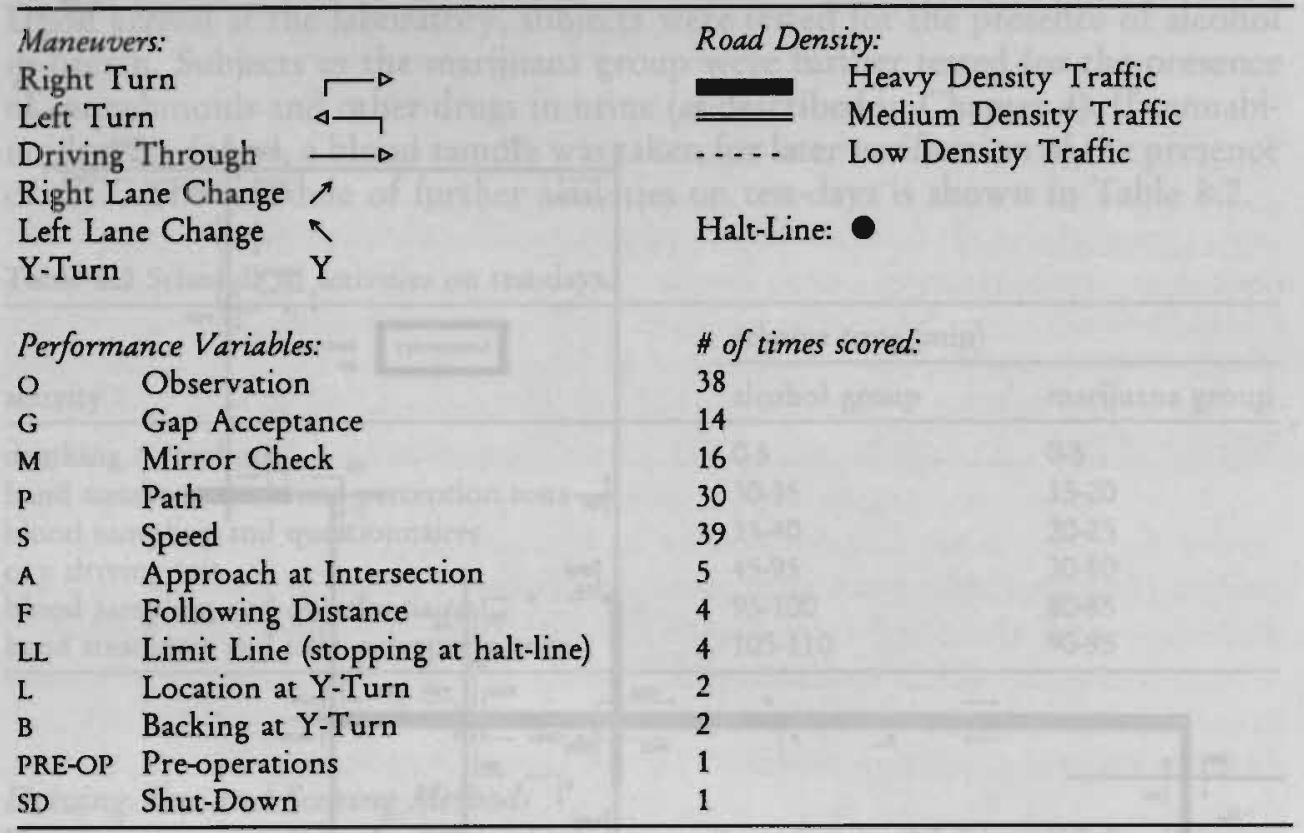

As described above, two different procedures were employed for determining a driver's proficiency from observer ratings; i.e. the molecular and the molar methods. The major difference between both methods is that performance variables are repeatedly measured on-line at different points along the route using the molecular approach; and, only once, retrospectively, in the molar approach.

The molecular approach was adopted from Jones (1978) who simplified the scoring of driving behavior so that the trained observer, sitting in the rear seat, attends to only one event at a time. The events observed at specific points along the route are only few aspects of total driving behavior. Thus, the observer is enabled to attend completely to the occurrence of each event in sequence while ignoring all other behavior which would in any case vary between tests. All aspects of a maneuver, such as a left turn, will be scored a number of times over the course of the whole route, but never more than a single aspect at a given moment.

Jones defined twelve aspects, called performance variables (Table 8.3), which were repeatedly scored, 156 times in total, at specific points along the route. The route was designed to fulfill Jones' requirements concerning maneuvers and road densities. The scoring sheet consisted of the schematic route map with symbols indicating the driver behavior to be scored at each point (reproduced in Figure 8.1 with symbology defined in Table 8.3). The observer moved his finger along the route and attended only to the behavior indicated, comparing it with a predefined standard of performance, and circled (if correct), crossed (if wrong) 
or underlined (if not observed) the symbol. If, for example, the observer were to score 'path' $(P)$ at a lane change $(\tau)$ the correct response was defined as: 1 . maintains straight path while scanning, 2. signals before the lane change, 3. steers smoothly with gradual angular movement, 4 . straightens vehicle in new lane, and 5. cancels signal. If the driver failed to make one or more of these responses 'path' was scored as fail, otherwise pass.

At the end of the test, the 'total score' was calculated by summing all passing items and dividing by the total number of observed items. Subscores were also calculated for 'observing', 'car control', 'judgment' and 'other' (Table 8.4). Three additional scores were obtained from the driving instructor; 1. 'instructor Control', the number of times the instructor took control, either orally or physically, 2. 'hazard Score', the number of times the driver responded correctly to hazards divided by the total number of hazards encountered, and 3. 'instructions', the number of times the driving instructor had to repeat a route instruction. All scores, except 'instructor control' and 'instructions', were expressed as per cent correct.

Table 8.4 Dependent variables, and their operationalization, measured by the molecular approach.

\begin{tabular}{|c|c|}
\hline dependent variable & operationalization \\
\hline $\begin{array}{l}\text { total score } \\
\text { observing }\end{array}$ & $\begin{array}{l}\% \text { correct of all } 156 \text { items } \\
\% \text { correct of } 68 \text { items comprising 'observation', 'gap acceptance', } \\
\text { and 'mirror check' }\end{array}$ \\
\hline $\begin{array}{l}\text { car control } \\
\text { judgment }\end{array}$ & $\begin{array}{l}\% \text { correct of } 69 \text { items comprising 'path' and 'speed' } \\
\% \text { correct of } 13 \text { items comprising 'approach', 'following distance' }\end{array}$ \\
\hline other & $\begin{array}{l}\% \text { correct of } 6 \text { items comprising 'Y-turn location and backing', } \\
\text { 'pre-operations' and 'shut-down' }\end{array}$ \\
\hline $\begin{array}{l}\text { hazard score } \\
\text { instructor control } \\
\text { instructions }\end{array}$ & $\begin{array}{l}\text { \% of correct responses to hazards encountered along the route } \\
\# \text { of times instructor took control, either orally or physically } \\
\# \text { of times the driving instructor had to repeat a route instruction }\end{array}$ \\
\hline
\end{tabular}

The molar approach required the driving instructor to retrospectively rate the driver's performance using a shortened version of the Royal Dutch Tourist Association's (ANWB) Driving Proficiency Test ${ }^{1}$. This instrument is normally applied for practical purposes; e.g. remedial training, driver's licensing, qualifying for a reduction in insurance premiums, etc. Items that did not apply to the driving test, e.g. those regarding railway crossing and special maneuvers like driving backwards and parking, were dropped from the normal list. In total, 108 items were dichotomously scored, as either pass or fail. Total test

${ }^{1} \mathrm{~A}$ copy of the test can be obtained from the author or the ANWB, The Hague. 
performance was measured by the percentage items scored as 'pass'. Subscores were calculated for 'vehicle checks', 'handling of vehicle', 'action in traffic', 'observation and understanding of traffic', and 'turning' (Table 8.5).

Table 8.5 Dependent variables, and their operationalization, measured by the molar approach.

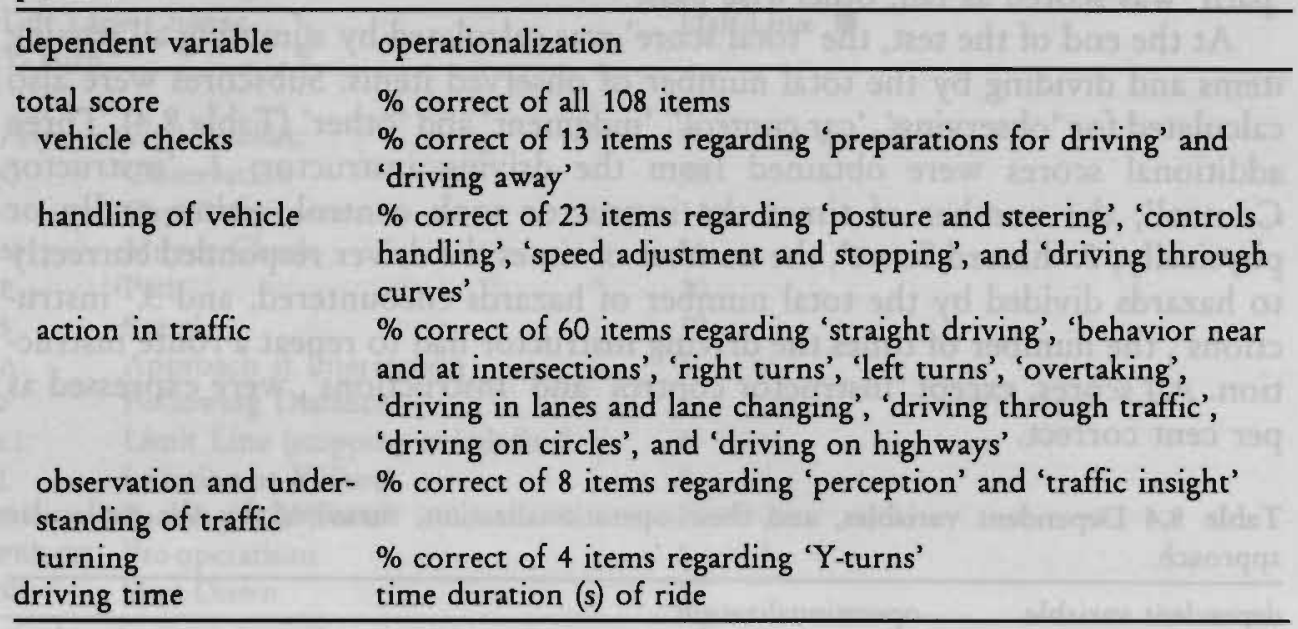

\section{Questionnaires}

Questionnaires used in the previous studies were administered to the subjects in the present study. Subjective feelings of intoxication ('high' or 'drunkness'), present cognitive and emotional state, and subjects' willingness to drive were assessed before the onset and after the conclusion of the driving tests. Subjects were also asked about their perception of the administered treatment, whether it was an active or placebo. At the end of each driving test, subjects were required to retrospectively rate the effort given in performing the test and perceived driving quality.

\section{Laboratory Tests}

Subjects performed two laboratory tests before and after driving. The hand steadiness test was used since it had shown a significant effect of the $100 \mu \mathrm{g} / \mathrm{kg}$ THC dose in the previous study and confirmation of this result would indicate the equivalent sensitivities of the present and previous groups to the drug. In addition a time perception test (method of interval production; Fraisse, 1963) was used to satisfy the investigator's curiosity about an oft-reported effect of THC. The former followed the same procedures as described in Chapter 7. The latter required the subjects to stand with eyes closed and indicate when he/she thought that 30 seconds had elapsed since a starting signal. The verbal response was timed to the nearest second by stop-watch. 


\section{Blood Sampling}

Blood samples were obtained by venepuncture immediately prior to and following all placebo and drug driving tests. Two aliquots containing $10 \mathrm{ml}$ each were heparinized and centrifuged, and the plasma fractions were placed in frozen storage for later assays to determine [THC] and [THC- $\mathrm{COOH}$, in the marijuana group; and, [ETOH], in the alcohol group. The analytical procedures regarding THC and THC-COOH assays were the same as in previous studies. As before, samples obtained in conjunction with placebo marijuana tests were only analyzed if the urine test had been positive. Plasma samples obtained from subjects in the alcohol condition were analyzed using gas-chromatography.

\section{Data Analysis}

For each variable measured on interval or ratio level, except plasma concentrations, change scores were computed by subtracting raw scores obtained in the placebo condition from those in the drug condition. In the figures, the mean change of the variable is depicted by the height of the bar and its standard error (SED) by the height of the vertical line above or below the bar. Change scores were expressed in absolute and not relative units. This means that if a performance measure fell from $80 \%$ after placebo to $70 \%$ after active drug, the change score would be $10 \%$ and not $12.5 \%$. Mean drug-placebo changes were tested for significant departure from zero by 2-tailed t-tests, for each group separately. Differences between the groups' mean changes were tested by 2-tailed t-tests for independent samples. Willingness to drive data were again analyzed for assessing differences between drug and placebo conditions using Cochran's Q-test. Differences between the respective groups' willingness to drive after drug treatments were analyzed by Mann-Whitney's U-test. Correlations were computed by Pearson's $r$ and tested for significant departure from zero by 2-tailed t-tests.

Preliminary analyses were executed to determine whether change scores were significantly different between subjects who received placebo in the first, and active drug in the second condition and those who received treatments in reversed order. Significant differences were generally absent and are therefore mentioned only in the exceptional cases where these were significant.

\subsection{Results}

\section{Plasma Concentrations of the Drugs}

Table 8.6 shows mean, median and range of $[\mathrm{THC}]$ and [THC-COOH] in the marijuana group and [ETOH] in the alcohol group. In the marijuana group, [THC] and [ $\mathrm{THC}-\mathrm{COOH}$ ] were in the expected range. In the alcohol group, mean [ETOH] at $t=35$ was somewhat lower than expected. Furthermore, the fall in [ETOH] to 
$t=95$ was less than expected from alcohol's pharmacokinetics; a fall of $.02 \%$ per hour is commonly seen during the elimination phase in subjects who fast prior to alcohol intake. This probably means that peak [ETOH] generally occurred during the driving test and was not much higher than measurements taken before and after. This situation contrasts to that for the subjects in the marijuana group whose [THC] reached its peak before the test and fell much more rapidly during it. Average plasma concentrations were significantly different from zero in all cases $(p<.001)$.

Table 8.6 Mean, median and range of [THC] and [THC-COOH] in $\mathrm{ng} / \mathrm{ml}(\mathrm{N}=14)$, and of [ETOH] in $\mathrm{g} \%(\mathrm{~N}=16)$.

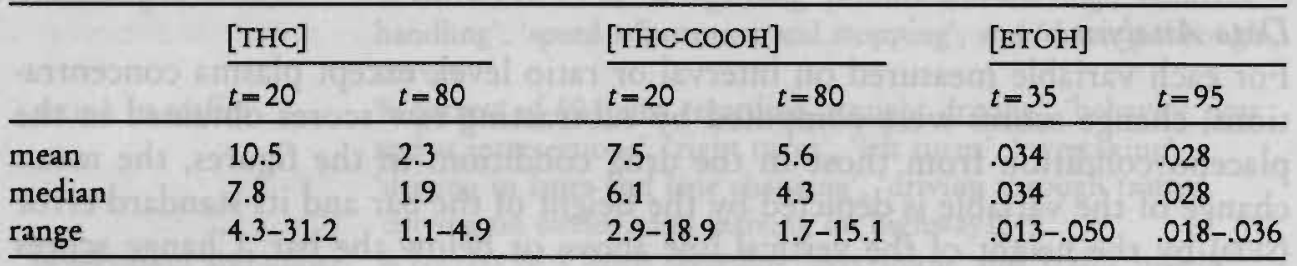

\section{Perceived 'bigh' and 'drunkness'}

Mean levels of intoxication reported by both groups varied from placebo to drug conditions in a remarkable manner. Few subjects in either group reported feeling intoxicated after placebo and their average levels were about $5 \%$ of maximum personal experience. After both THC and alcohol these levels rose to about $35 \%$ when the respective groups were about to begin driving, then declined to $25 \%$ at the end of driving. Means and standard errors of differences (SED) in reported intoxication are shown in Figure 8.2.

T-tests showed that ratings of intoxication after active drugs were significantly different from zero in the marijuana and alcohol groups, both before $\left(T_{13}=4.36\right.$ $\& T_{15}=5.20$, respectively; $\left.p<.001\right)$ and after driving $\left(T_{13}=3.44 \& T_{15}=5.23\right.$, respectively; $p<.001$ ).

Subjects' responses to the question of whether they thought the administered drug was active or placebo showed that they were well aware of what they smoked or drunk. In the marijuana group, $93 \%$ of the subjects correctly identified the placebo cigarette when it was administered, and $79 \%$, the active drug. In the alcohol group, $94 \%$ of the subjects correctly identified the placebo alcohol when it was administered, and $87 \%$, the active drug.

\section{Driving Performance Measured by the Molecular Approach}

Though some of the changes were positive, indicating better performance after drug and some were negative, showing the opposite, no mean change was significant (Table 8.7). Differences between the two groups' changes were also 


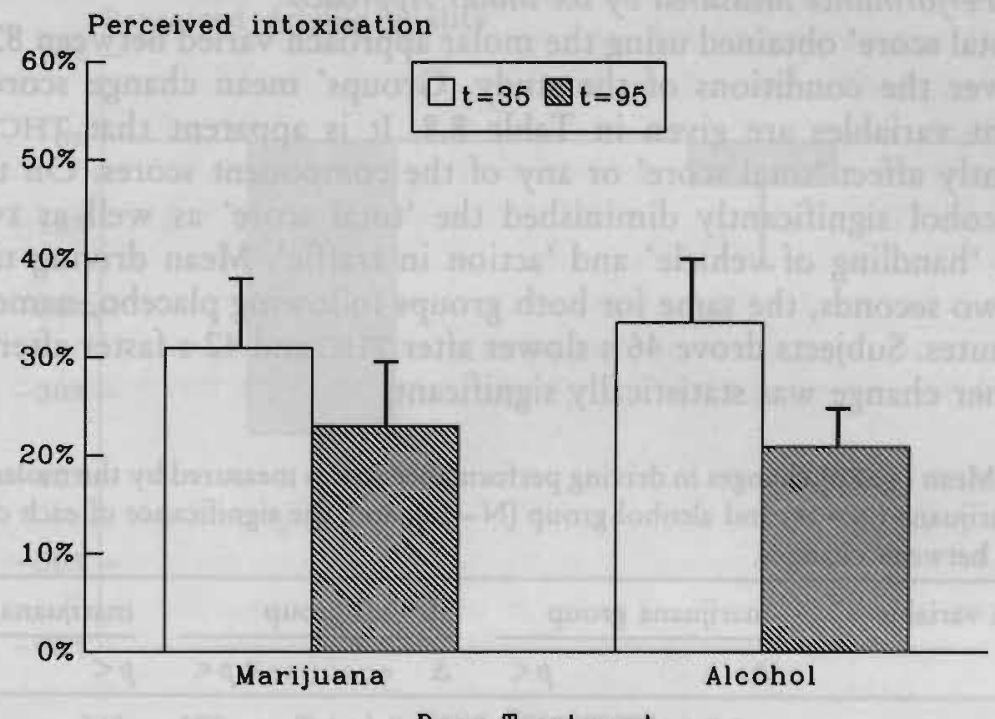

Drug Treatment

Figure 8.2 Mean (+SED) changes in perceived intoxication by Drug and Time, relative to placebo. Raw scores were expressed as percentage of maximum personal experience.

not significant, although one came close: the difference between improvement shown by the marijuana group and impairment shown by the alcohol group in 'car control' approached significance $(p<.059)$. In general, the effects of both drugs on driving performance, as measured by the molecular approach were very small or absent.

Table 8.7 Mean ( \pm SED) changes in driving performance scores measured by the molecular approach for the marijuana $(\mathrm{N}=14)$ and alcohol group $(\mathrm{N}=16)$; and, the significance of each change and difference between changes.

\begin{tabular}{|c|c|c|c|c|c|}
\hline \multirow[t]{2}{*}{ dependent variable } & \multicolumn{2}{|c|}{ marijuana group } & \multicolumn{2}{|l|}{ alcohol group } & \multirow{2}{*}{$\frac{\text { marijuana } v s \text { alcohol }}{p<}$} \\
\hline & $\Delta$ & $p<$ & $\Delta$ & $p<$ & \\
\hline total score & $+1.6( \pm 1.7)$ & ns & $-1.5( \pm 1.7)$ & ns & ns \\
\hline observing & $+2.4( \pm 3.1)$ & ns & $-0.3( \pm 2.8)$ & ns & ns \\
\hline car control & $+2.7( \pm 2.1)$ & ns & $-2.6( \pm 1.8)$ & ns & .059 \\
\hline judgment & $-8.3( \pm 4.8)$ & ns & $-2.2( \pm 4.0)$ & ns & ns \\
\hline other & $-3.6( \pm 4.3)$ & ns & $+1.3( \pm 4.4)$ & ns & ns \\
\hline hazard score & $+6.3( \pm 17.6)$ & ns & $+14.6( \pm 9.4)$ & ns & ns \\
\hline instructor contr & $+0.5( \pm 0.4)$ & ns & $+0.4( \pm 0.5)$ & ns & ns \\
\hline instructions & $+0.1( \pm 0.6)$ & ns & $+0.8( \pm 0.6)$ & ns & ns \\
\hline
\end{tabular}




\section{Driving Performance Measured by the Molar Approach}

Mean 'total score' obtained using the molar approach varied between $82.8 \%$ and $89.5 \%$ over the conditions of the study. Groups' mean change scores on all dependent variables are given in Table 8.8. It is apparent that THC did not significantly affect 'total score' or any of the component scores. On the other hand, alcohol significantly diminished the 'total score' as well as two components, 'handling of vehicle' and 'action in traffic'. Mean driving time was, within two seconds, the same for both groups following placebo, namely about $41 \frac{1}{2}$ minutes. Subjects drove $46 \mathrm{~s}$ slower after THC and $42 \mathrm{~s}$ faster after alcohol, but neither change was statistically significant.

Table 8.8 Mean ( \pm SED) changes in driving performarice scores measured by the molar approach for the marijuana $(\mathrm{N}=14)$ and alcohol group $(\mathrm{N}=16)$; and, the significance of each change and difference between changes.

\begin{tabular}{|c|c|c|c|c|c|}
\hline \multirow[t]{2}{*}{ dependent variable } & \multicolumn{2}{|c|}{ marijuana group } & \multicolumn{2}{|l|}{ alcohol group } & $\underline{\text { marijuana vs alcohol }}$ \\
\hline & $\Delta$ & $p<$ & $\Delta$ & $p<$ & $p<$ \\
\hline $\begin{array}{l}\text { total score } \\
\text { vehicle checks } \\
\text { handling of vehicle } \\
\text { action in traffic } \\
\text { observation and under- } \\
\text { standing of traffic }\end{array}$ & $\begin{array}{l}-0.7( \pm 2.7) \\
-0.6( \pm 1.5) \\
+3.7( \pm 2.8) \\
-2.7( \pm 3.1) \\
+1.8( \pm 8.7)\end{array}$ & $\begin{array}{l}\text { ns } \\
\text { ns } \\
\text { ns } \\
\text { ns } \\
\text { ns }\end{array}$ & $\begin{array}{l}-6.8( \pm 1.8) \\
+0.5( \pm 1.3) \\
-8.4( \pm 2.2) \\
-8.4( \pm 2.3) \\
-6.3( \pm 7.0)\end{array}$ & $\begin{array}{l}.002 \\
\text { ns } \\
.002 \\
.003 \\
\text { ns }\end{array}$ & $\begin{array}{l}.065 \\
\mathrm{~ns} \\
.002 \\
\mathrm{~ns} \\
\mathrm{~ns}\end{array}$ \\
\hline $\begin{array}{l}\text { turning } \\
\text { driving time }\end{array}$ & $\begin{array}{l}-1.8( \pm 4.9) \\
+45.6( \pm 51.8)\end{array}$ & $\begin{array}{l}\text { ns } \\
\text { ns }\end{array}$ & $\begin{array}{l}+3.1( \pm 7.5) \\
-42.0( \pm 32.4)\end{array}$ & $\begin{array}{l}\text { ns } \\
\text { ns }\end{array}$ & $\begin{array}{l}\text { ns } \\
\text { ns }\end{array}$ \\
\hline
\end{tabular}

Differences between the groups' mean change scores were also significant or nearly so. The greater drop in 'total score' caused by alcohol was almost significantly different from that caused by THC $(p<.065)$. The difference between groups' changes in 'handling of vehicle', a component score, was significant $(p<.002)$ : whereas the marijuana group's performance improved slightly, that of the alcohol group deteriorated under the influence of their respective drugs. In particular, 6 items checked under the category of 'handling of vehicle' discriminated between the groups' reactions to the drugs. These all pertained to how well the driver handled the vehicle through curves. Whereas THC had little effect on this ability, alcohol seemed to affect it strongly. This more than any other factor was responsible for the difference between the instructor's ratings of how the two groups were affected by the respective drugs.

\section{Perceived Driving Quality and Effort to Accomplish the Test}

Both groups rated their driving performance following placebo as somewhat better than 'normal'. Following the active drug, ratings were about $35 \%$ lower in the marijuana group, but only $5 \%$ lower in the alcohol group (Figure 8.3). 


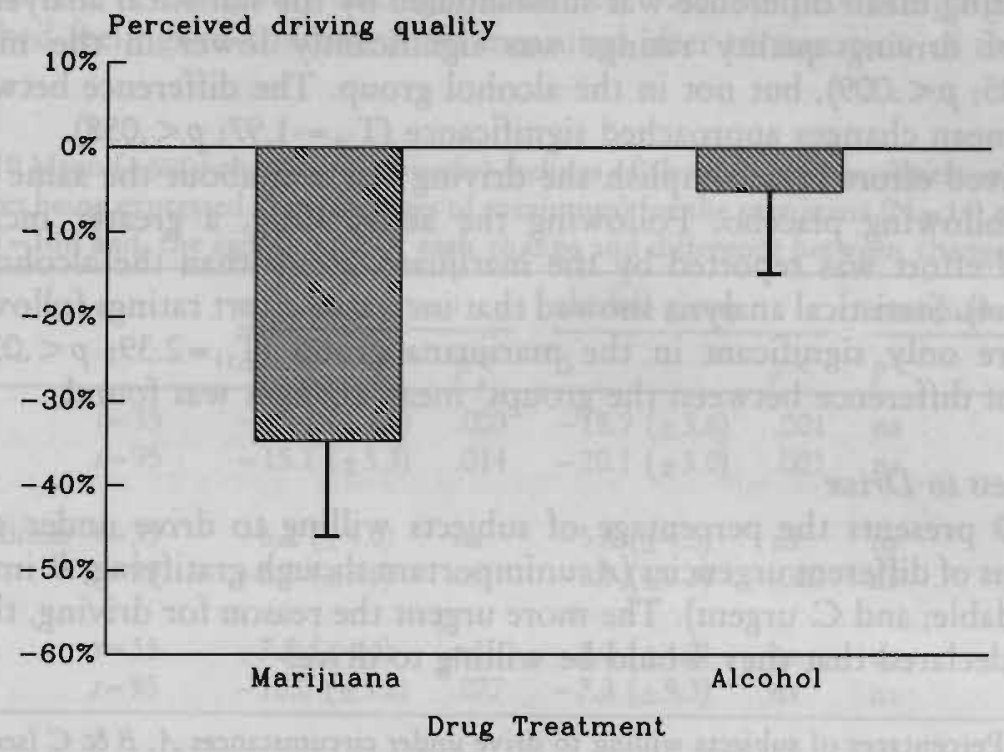

Figure 8.3 Mean (+SED) changes in perceived driving quality by Drug, relative to placebo. Raw scores were expressed as percentage of 'normal'.

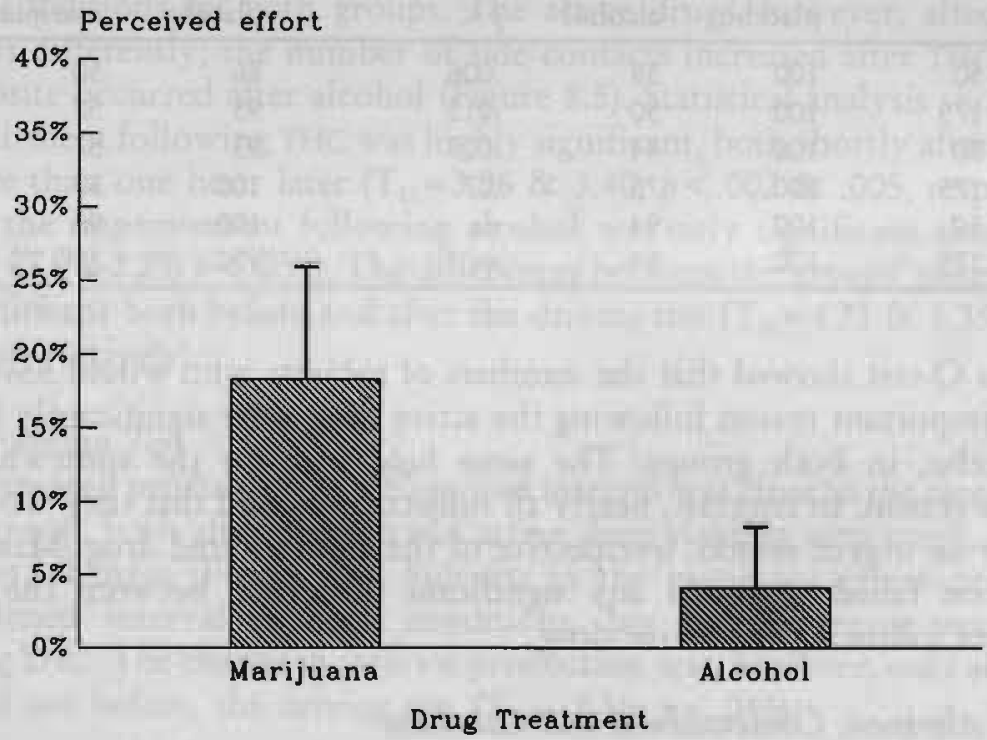

Figure 8.4 Mean (+SED) changes in perceived effort to accomplish the driving test by Drug, relative to placebo. Raw scores were expressed as percentage of maximum of scale. 
This striking mean difference was substantiated by the statistical analysis. Mean change of driving quality ratings was significantly lower in the marijuana $\left(\mathrm{T}_{13}=-3.05 ; p<.009\right)$, but not in the alcohol group. The difference between the groups' mean changes approached significance $\left(\mathrm{T}_{28}=-1.97 ; p<.058\right)$.

Perceived effort to accomplish the driving test was about the same in both groups following placebo. Following the active drug, a greater increase in perceived effort was reported by the marijuana group than the alcohol group (Figure 8.4). Statistical analysis showed that increased effort ratings following the drug were only significant in the marijuana group $\left(T_{13}=2.39 ; p<.033\right)$. No significant difference between the groups' mean changes was found.

\section{Willingness to Drive}

Table 8.9 presents the percentage of subjects willing to drive under specified conditions of different urgencies ( $A$. unimportant though gratifying; $B$. important but avoidable; and $C$. urgent). The more urgent the reason for driving, the more subjects declared that they would be willing to drive.

Table 8.9 Percentages of subjects willing to drive under circumstances $A, B \& C$ (see text) by treatment condition and sampling time; and, the significance of each difference between placebo and active drug condition, tested by Cochran's Q-test.

\begin{tabular}{lllllllll}
\hline & & \multicolumn{3}{l}{ alcohol group } & & & \multicolumn{3}{c}{ marijuana group } \\
\cline { 3 - 4 } \cline { 7 - 9 } & & placebo & alcohol & $p<$ & & placebo & marijuana & $p<$ \\
\hline A & $t=50$ & 100 & 38 & .006 & & 86 & 50 & .044 \\
& $t=175$ & 100 & 50 & .012 & & 93 & 57 & .044 \\
B & $t=50$ & 100 & 44 & .008 & & 93 & 57 & .044 \\
& $t=175$ & 100 & 75 & .07 & & 100 & 57 & .03 \\
$\mathrm{C}$ & $t=50$ & 100 & 94 & $\mathrm{~ns}$ & & 100 & 93 & $\mathrm{~ns}$ \\
& $t=175$ & 100 & 94 & $\mathrm{~ns}$ & & 100 & 100 & $\mathrm{~ns}$ \\
\hline
\end{tabular}

Cochran's Q-test showed that the numbers of subjects who would have driven for an unimportant reason following the active drug were significantly less than after placebo, in both groups. The same held true for the somewhat more important reason. In contrast, nearly all subjects indicated that they would have driven for an urgent reason, irrespective of the administered drug. Mann-Whitney's U-test failed to reveal any significant difference between the groups' percentages following the active drug.

\section{Perceived Alertness, Contentedness and Calmness}

Changes in subjective feelings of alertness, contentedness and calmness are shown in Table 8.10. Feelings of alertness were significantly diminished in both groups and at both time points following the active drug relative to placebo. Subjects felt also less content and calm following the active drugs, but the effects were smaller. 
Statistical tests revealed only one significant effect: alcohol producing significantly diminished feelings of contentedness measured after termination of the driving test.

Table 8.10 Mean ( \pm SED) changes in subjective feelings of alertness, contentedness and calmness (raw scores being expressed as percentages of maximum) for the marijuana $(\mathrm{N}=14)$ and alcohol group $(N=16)$; and, the significance of each change and difference between changes.

\begin{tabular}{|c|c|c|c|c|c|c|}
\hline & & \multicolumn{2}{|c|}{ marijuana group } & \multicolumn{2}{|l|}{ alcohol group } & \multirow{2}{*}{$\frac{\text { marijuana vs alcohol }}{p<}$} \\
\hline & & $\Delta$ & $p<$ & $\Delta$ & $p<$ & \\
\hline Alertr & $\begin{array}{l}t=35 \\
t=95\end{array}$ & $\begin{array}{l}-15.4( \pm 4.2) \\
-15.1( \pm 5.3)\end{array}$ & $\begin{array}{l}.003 \\
.014\end{array}$ & $\begin{array}{l}-18.9( \pm 3.6) \\
-20.1( \pm 3.0)\end{array}$ & $\begin{array}{l}.001 \\
.001\end{array}$ & $\begin{array}{l}\text { ns } \\
\text { ns }\end{array}$ \\
\hline Contentedness & $\begin{array}{l}t=35 \\
t=95\end{array}$ & $\begin{array}{l}-6.2( \pm 4.0) \\
-5.7( \pm 3.6)\end{array}$ & $\begin{array}{l}\text { ns } \\
\text { ns }\end{array}$ & $\begin{array}{l}-5.8( \pm 4.3) \\
-8.9( \pm 2.3)\end{array}$ & $\begin{array}{l}\text { ns } \\
.002\end{array}$ & $\begin{array}{l}\text { ns } \\
\text { ns }\end{array}$ \\
\hline Calmness & $\begin{array}{l}t=35 \\
t=95\end{array}$ & $\begin{array}{l}-7.5( \pm 4.4) \\
-10.0( \pm 5.2)\end{array}$ & $\begin{array}{l}\text { ns } \\
.077\end{array}$ & $\begin{array}{l}-1.2( \pm 4.2) \\
-7.3( \pm 5.3)\end{array}$ & $\begin{array}{l}\text { ns } \\
\text { ns }\end{array}$ & $\begin{array}{l}\text { ns } \\
\text { ns }\end{array}$ \\
\hline
\end{tabular}

\section{Hand Steadiness Test}

Mean square root of total number of side contacts were about the same in the placebo conditions for both groups. The active drugs, however, affected hand steadiness differently; the number of side contacts increased after THC whereas the opposite occurred after alcohol (Figure 8.5). Statistical analysis showed that the impairment following THC was highly significant, both shortly after smoking and more than one hour later $\left(T_{13}=3.96 \& 3.40 ; p<.002 \& .005\right.$, respectively), whereas the improvement following alcohol was only significant shortly after drinking $\left(\mathrm{T}_{15}=-2.29 ; p<.037\right)$. The differences between the groups' mean changes were significant both before and after the driving test $\left(T_{28}=4.71 \& 3.35 ; p<.001\right.$ $\& .002$, respectively).

\section{Time Perception Test}

Mean attempted production of a 30-second interval was close to the target for the alcohol group, both after placebo and active drug yielding very small, and nonsignificant, changes (Figure 8.6). Subjects in the marijuana group produced a foreshortened interval in both conditions, but foreshortening was greater following THC. The change in interval production was, however, only significant after, and not before, the driving test $\left(\mathrm{T}_{13}=-2.36 ; p<.045\right)$. 


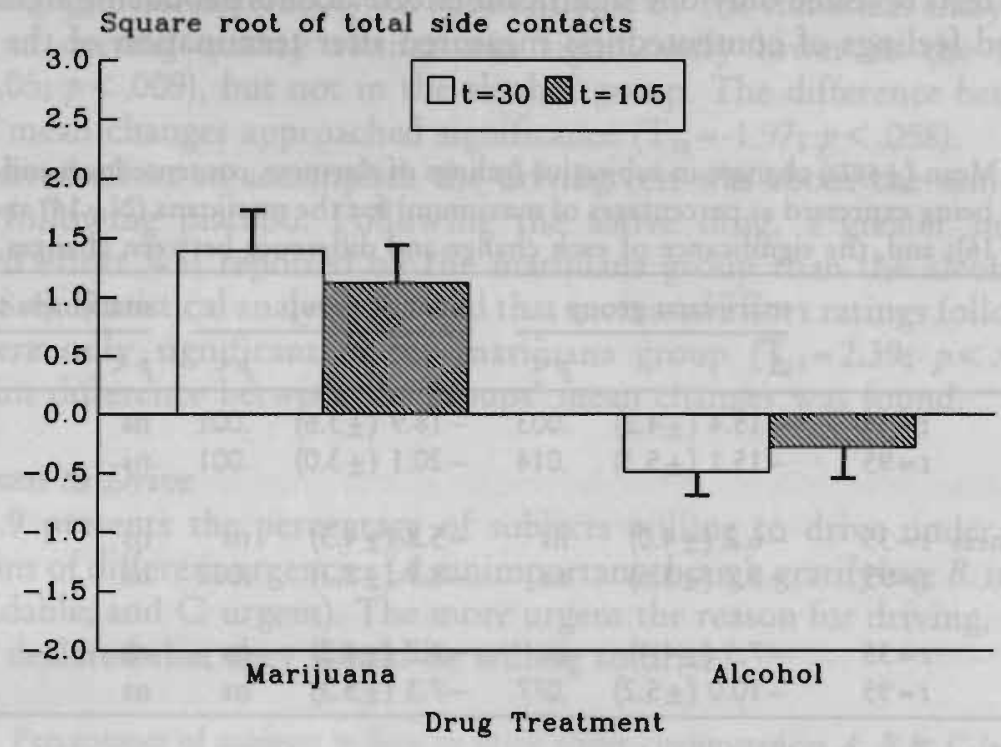

Figure 8.5 Mean (+SED) changes in the square root of total number of side contacts in the hand steadiness test by Drug, relative to placebo.

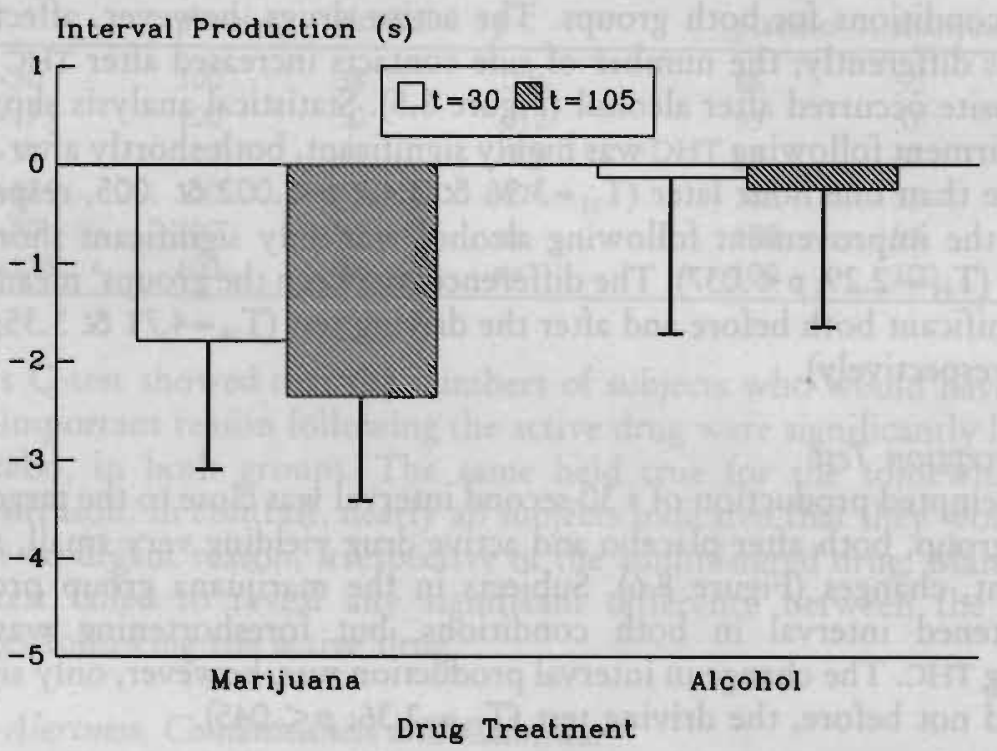

Figure 8.6 Mean (+SED) changes in attempted production of a 30-second interval by Drug, relative to placebo. 
Inter-Subject Relations between Variables

Relationship between Plasma Concentrations and Driving Performance. Correlational analyses were performed to determine whether driving performance was related to plasma concentrations of the drugs, measured both before and after the driving tests. The first step involved correlational analysis between raw variables; the second step, between changes in driving performance and plasma concentrations. Neither analysis yielded significant results.

Relationship between the Molecular and Molar Approach. Correlations were also computed between the raw 'total scores' obtained by both the molecular and molar approach. These correlations were computed in each condition separately. For the marijuana group, the correlation between the Total Scores were 0.71 $(p<.005 ; 2$-tailed $)$ and $0.54(p<.044)$ in the placebo and active drug condition, respectively. For the alcohol group, the corresponding correlations were 0.71 $(p<.002)$ and $0.79(p<.001)$, respectively. Differences between these correlations were not significant. The second step involved correlational analysis of change scores. This analysis revealed that changes in 'total scores' of both methods were not significantly correlated, neither in the marijuana $(r=0.05)$ nor in the alcohol group $(r=0.08)$.

Relationship between Driving Performance and Driving Experience. Correlations were also computed between driving experience on one hand, and driving performance and changes in driving performance following the active drug on the other. Neither analysis revealed significant correlations. This means that driving experience of the subjects in the present study could neither predict driving performance per se nor changes following the active drug.

Relationship between Plasma Concentrations and Perceived Intoxication. Correlations of perceived 'high' following THC with [THC] were $0.65 \quad(p<.013)$ shortly after smoking and $0.47(p<.093)$ following termination of the driving test. Correlations with [THC-COOH] were $0.74(p<.002)$ and $0.62(p<.018)$, respectively. Correlations of perceived intoxication following alcohol and [ETOH] were not significant $(r=0.27 \& 0.39$, respectively). Correlations of plasma concentrations with changes in perceived intoxication were nearly the same as those with the raw scores, because intoxication ratings were generally zero following placebo.

\subsection{Discussion}

The present study showed that alcohol, administered in a dose of $0.43 \mathrm{~g}$ ethanol per $\mathrm{kg}$ of lean body mass yielding an average plasma alcohol concentration of 
about $0.04 \mathrm{~g} \%$, produced a significant impairment in city driving as measured by the molar approach, relative to placebo. Significant impairment was shown by changes in 'total score', and subscores describing 'handling of vehicle' and 'action in traffic'. Marijuana, administered in a dose of $100 \mu \mathrm{g}$ THC per $\mathrm{kg}$ of whole body weight, on the other hand, did not significantly change mean driving performance as measured by this approach. Neither alcohol nor marijuana significantly affected driving performance measures obtained by the molecular approach.

The first conclusion one might draw is that the methods applied for measuring driving performance did not yield exactly the same results. Yet both methods similarly measured individual differences in driving proficiency. Correlations between the total scores in all four conditions were between 0.54 and 0.79 , and as such, highly significant. Obviously both methods were able to discriminate the relatively large pre-existing differences in the subjects' respective abilities to operate the vehicle in urban traffic. Change scores were, however, not correlated; i.e. $r=0.05 \& 0.08$ in the marijuana and alcohol group, respectively. Since the molar, and not the molecular, approach was sensitive to alcohol's adverse effects, it is tempting to conclude that the latter may fail to measure some important driving deficits. That is, the molecular approach seems relatively insensitive to drug-induced changes.

The second conclusion one might draw is that the molar method, having proven its sensitivity by detecting impairment associated with a low BAC, should be sensitive to any similar impairment occurring after a correspondingly low THC dose. That it failed to show any significant change in the marijuana group's driving performance leads to the third and most important conclusion:

The $100 \mu \mathrm{g} / \mathrm{kg}$ THC dose these subjects received shortly before the test did not impair their city driving performance

The different effects of alcohol and marijuana on driving performance can not be explained by the subjects' reported driving experience. Although subjects in the alcohol group were somewhat more experienced drivers than those in the marijuana group, the difference was not significant. Furthermore, correlational analysis showed that neither driving performance nor driving impairment was significantly related to previous driving experience. The different effects of alcohol and marijuana on driving performance can also not be explained by differences in the groups' subjective feelings. There was a remarkable correspondence between the two groups' feelings of intoxication, i.e. 'high' or 'drunkness'. Prior to the driving test, both groups reported intoxication levels of about $35 \%$ of the highest ever experienced, and 25\%, after its termination. THC's significant impairing effect on hand steadiness also showed that the subjects in the marijuana group were sensitive to THC's effects. 
The difference between the drugs' effects on driving may be explained, however, by compensational mechanisms, such as increased effort. Both groups reported about the same amount of effort in accomplishing the driving test following placebo. Yet only subjects in the marijuana group reported significantly higher levels of invested effort following the active drug. Previous on-road driving studies indicate that subjects may compensate for THC's adverse effects by slowing down (Hansteen et al., 1976; Casswell, 1979; Peck et al., 1986). Results of the present study were in the same direction: it took the marijuana group an average of $46 \mathrm{~s}$ longer to complete the circuit after THC than following placebo. That this difference was not significant was probably due to the real-life character of the test: unforseen obstructions and variable traffic density probably increased the error variance of this measure over what it would have been on a closed course. Thus, there was evidence that subjects in the marijuana group were not only aware of their intoxicated condition but were also attempting to compensate for it.

There were other striking differences between the groups' reactions to their respective drugs. As stated, subjects in the marijuana group invested more effort in the test while driving under the influence of THC. The alcohol group apparently made no greater effort after the drug than placebo. Yet for all of their effort, the marijuana group rated their driving performance as being significantly worse after THC than placebo. The alcohol group who invested no special effort after the drug, not surprisingly, thought they had driven as well as following placebo. They were nonetheless driving in a deficient manner according to the instructor's ratings. These seem to be important findings. They support both the common belief that drivers become overconfident after drinking alcohol and investigators' suspicions that they become more cautious and self-critical after consuming low THC doses by smoking marijuana.

These impressions were seemingly contradicted by the similarity in the groups' responses indicating their willingness to drive after the respective drugs. About $50 \%$ of both groups said they would have been unwilling to drive for less than very urgent reasons. This profession of caution seems a bit odd for the alcohol group who knew that they could not be given a dose which would produce BACs over the legal limit during the test. The 'contradiction' in this case seems to be a reflection of the group's desire to give 'socially desirable' answers to the questionnaire, whether the same was true for the marijuana group in the present and preceding studies is a moot point.

The laboratory performance tests also discriminated between the drugs' effects. Hand steadiness was impaired following THC and improved following alcohol, relative to placebo. The difference between the drugs' effects was significant, both before and after the driving test. Impairment after THC was about as much as that produced by the same dose in the previous study, indicating equivalent sensitivities of the present and previous groups. Production 
of time intervals was not affected by alcohol, but THC significantly shortened interval production, relative to placebo. This confirms the common finding that THC affects time estimation (Hollister and Gillespie, 1970; Bech et al., 1973). As usual, THC accelerated the subjects" perception of time passing. They arrived at a foreshortened production of $30 \mathrm{~s}$ and would have estimated the duration of the actual interval as longer that it actually was. Changes in driving performance were not correlated with changes in performance in either laboratory test. Nevertheless, both tests should be seriously considered for inclusion in any test battery for measuring these drugs' effects, mainly because of the different pattern of results they produced.

Plasma concentrations of THC and THC-COOH were comparable to those obtained in the previous studies. Plasma concentrations of ethanol were somewhat lower than predicted, and the fall in plasma concentration during, the driving test was less than expected. This probably means that peak plasma concentrations generally occurred during the driving test. If so, it probably was about $0.04 \mathrm{~g} \%$. Ratings of 'high' were significantly related to plasma concentrations of THC and THC-COOH but those of 'drunkness' were not significantly related to plasma concentrations of ethanol. Drug plasma concentrations were neither related to absolute driving performance scores nor to the changes that occurred from placebo to drug conditions. With respect to THC, these results confirm the findings in previous studies. They are somewhat surprising for alcohol but may be due to the restricted range of ethanol concentrations in the plasma of different subjects.

How do the present results relate to those reported by Klonoff (1974)? $\mathrm{He}$ also assessed the effects of relatively low THC doses on aspects of subjects' driving performance in a city driving test using a similar molar approach. His subjects commenced driving immediately after cessation of smoking. They scored lower on judgment and concentration scales when under the influence of the highest, but not the lowest, dose. Behaviors that were more directly related to driving performance, e.g. lane changing, compliance with traffic signals and posted speed advisories, were not affected by the drug. Subjects in the present study commenced driving 30 minutes after initiation of smoking. The molar method applied in the present study did not include ratings of judgment or concentration. With the exception of these two items, neither study indicated significant effects of THC on driving performance and are, therefore, in complete agreement. 


\section{CHAPTER 9}

\section{GENERAL DISCUSSION}

\subsection{Introduction}

It is commonly assumed that cannabis smoking in real-life social situations delivers THC doses that seriously degrade the ability to safely operate motor vehicles; and, that the drug's users frequently drive shortly after smoking. If these premises are correct, it would follow that cannabis users are, while intoxicated, at increased risk of traffic accident involvement and constitute a safety hazard for other road users. However, the foremost impression one gains from reviewing the literature is that no clear relationship has ever been demonstrated between cannabis smoking and either seriously impaired driving performance or traffic safety. The epidemiological evidence, as limited as it is, shows that the combination of THC and alcohol is over-represented in injured and dead drivers and more so in those who actually caused the accidents. Yet there is little if any evidence to indicate that drivers who have used cannabis alone are any more likely to cause serious accidents than drug free drivers. To a large extent, the results from driving simulator and closed-course tests corroborate the epidemiological findings by indicating that THC in single inhaled doses up to about $250 \mu \mathrm{g} / \mathrm{kg}$ has relatively minor effects on driving performance, certainly less than $\mathrm{BACs}$ in the range $0.08-0.10 \mathrm{~g} \%$.

But how well do these findings relate to the actual driving performance of regular cannabis users? If previous experience is any guide, little of crucial importance will emerge from experimental research until it is conducted in a more realistic manner. Therefore, a series of studies were conducted to determine the dose-response relationship between THC and objectively and subjectively measured aspects of real world driving. A variety of driving tasks were employed, including maintenance of a constant speed and steady lateral position during uninterrupted highway travel, adjusting velocity to the movements of a leading car on a highway, and city driving. The purpose of applying different tests was to determine whether similar changes in performance under the influence of THC occurs in all thereby indicating a general drug effect on driving safety. 
However real these tests might appear, the circumstances in which the subjects smoked the drug and drove the car were still somewhat artificial. First, the subjects consumed the drug in a neutral setting, alone or in the presence of a stranger. This was of course different from the situation wherein they normally smoke cannabis, i.e. in the company of friends who might reinforce a certain type of behavior not normally considered as conservative or prudent. Marks and Pow (1989) found that marijuana smokers derived slightly greater pleasure from THC in the company of friends than strangers from which one might infer a social amplification of the pharmacological effect. However, neither they nor any other investigators ever indicated that social amplification outlasts the situation which gives rise to it. In the absence of such evidence, we tend to discount the possibility that the unusual setting for drug administration had any effect upon subsequent driving performance. Social amplification of THC's adverse effects on driving performance might well occur if the driver were accompanied by similarly intoxicated passengers. We must therefore restrict generalizations from the studies' results to those situations where the intoxicated driver is doing his best to perform efficiently and is uninfluenced by friends or any other factor which might amplify the drug's adverse effects.

Secondly, subjects were aware of the fact that their driving behavior was being observed and that the accompanying driving instructor would intervene if necessary. The former is hardly avoidable in experimental studies but possibly provoked the subjects to do their utmost; the latter is a prerequisite for ensuring safety in actual driving studies and may or may not have produced nonchalance on the subjects' part resulting in poorer than normal performance. Yet according to what the subjects declared, they drove normally after placebo indicating that the experimental situation had not seriously altered their performance. Whether they drove in the test as they normally would after smoking cannabis remains open to question. The correspondence between experimental and epidemiological studies regarding THC's effects on driving performance suggests that this artificiality is also of minor concern.

The present series of studies are about the best simulation of real world driving one can reasonably achieve and have gone further toward defining the effects of cannabis smoking on actual driving performance than any of its predecessors. The results found in the successive studies were discussed at full length in the respective chapters. In this chapter, results of the separate studies will be integrated to provide answers to some important questions concerning marijuana's influence on driving performance. First however, the implications of the laboratory study's major result will be discussed, i.e. the THC dose that cannabis users prefer to achieve their desired 'high'. In the following section, the driving tests will be discussed in terms of the kinds of mental operations they require. Some further remarks provided in that section concern the relevance of the driving tests for traffic safety. The major part of this chapter will be ad- 
dressed to the most important issue, i.e. what the results indicate as the real effects of marijuana smoking on driving performance. This chapter will end with a list of conclusions and recommendations.

\subsection{THC Doses}

To avoid arbitrarily selecting an unrealistic maximum THC dose for the driving studies, it was necessary to establish the dose that cannabis users actually prefer for achieving their desired 'high'. A laboratory study met this requirement. The study showed that cannabis users prefer higher THC doses than those previously administered in experimental studies. Previous THC doses were generally between 100 and $200 \mu \mathrm{g} / \mathrm{kg}$, including the remaining butt, whereas the subjects in the laboratory study preferred an average dose of $308 \mu \mathrm{g} / \mathrm{kg}$, excluding the butt. This either means that today's cannabis users prefer higher doses than in the past or that investigators failed to administer realistic doses in previous studies, or both.

Two previous studies employed similar procedures as the present laboratory study to determine the THC dose that marijuana users prefer. Cappell et al. (1973) allowed subjects to smoke as many marijuana cigarettes, containing either 0.2 , 0.4 , or $0.8 \% \mathrm{THC}$, as they needed to achieve a 'nice high'. Comparing the weight of the remaining butt with the unlit cigarette revealed that the average THC consumption was inversely related to drug potency: with increasing potency, the twelve males participating in the study smoked $3.8,6.0$, and $11.0 \mathrm{mg} \mathrm{THC}$, respectively. The investigators failed, however, to report the subjects' mean weight which makes it difficult to compare the results with those of the present study. Cappell and Pliner (1974) replicated the study with potencies of $0.36,0.73$, and $1.45 \%$, and reported the average amounts of marijuana consumed by the sixty males participating in the study: they were $21.36,15.57$, and $13.34 \mathrm{mg} / \mathrm{kg}$ for the low, medium, and high potencies, respectively. Assuming that THC was equally distributed over the entire cigarettes, this means that the subjects smoked 77,114 , and $193 \mu \mathrm{g} / \mathrm{kg}$ THC, respectively. The potency of the cigarettes used in the present laboratory study was $2.57 \%$ and our subjects smoked on the average a THC dose of $308 \mu \mathrm{g} / \mathrm{kg}$. Assuming that the smoking technique did not change as a function of drug potency (for a discussion, see Section 2.5.2), these results suggest that the potency of the marijuana cigarette contributes to the preferred dose, at least if the potency is in the range of $0.2-2.5 \%$. It is therefore hard to say whether current users indeed prefer higher doses than almost two decades ago or that they simply have been unable to sufficiently discriminate among the administered potencies. What is clear from the laboratory study's results, is that higher THC doses than those usually administered in previous studies should be included in future ones. 
The relation between preferred dose and the potency of the drug raises two questions that might have implications for interpreting the present studies' results. The first question is, how do people regulate their THC consumption? Apparently they do not titrate brain THC concentrations to within a narrow range as tobacco smokers reliably do with nicotine (Herning et al., 1981; Gust and Pickens, 1982). It's also doubtful that marijuana smokers integrate their THC concentrations over time to cease consumption when the cumulative effect approaches a certain threshold. The probable explanation is that our subjects were somewhat aware of both the momentary brain concentration and its rate of increase and ceased smoking before the expected rise would shortly exceed the preferred effect. This procedure was accurate enough to limit the drug's effects to below what they had previously experienced as an unpleasant maximum. It was not accurate enough to attain homogeneity in the subjects' achievement of the preferred 'high' in relation to the maximum (coefficient of variation: $25 \%$ ). In real life, cannabis users are usually more aware of the potency of the material they smoke, from prior experience, upon the advice of other users or even from its 'street' price. The material presented to the subjects in the laboratory study was definitely unfamiliar and they may have suddenly become prudent smokers. Confirmation of this supposition came from the same subjects' consumption of THC in the subsequent driving study. Though treated with the same average dose they had preferred in the laboratory study, their plasma drug concentrations were now higher. This difference was interpreted as the result of increased smoking efficiency due to familiarization with the potency of the material containing the drug.

If familiarization plays a major role in smoking efficiency and, consequently THC plasma concentrations, THC's adverse effects on performance may also be dependent on this factor. That being the case, the second and third driving studies' results would under-estimate THC's adverse effects on driving performance since new groups of subjects were recruited in both. However, the remarkable resemblance between effects of every THC dose on SDLP in the first and second driving studies indicates that familiarization is not a very important factor. Nevertheless, we recommend that subjects in future studies be familiarized with the potency of the drug before the beginning of performance testing.

The second question raised by the apparent relationship between the drug's potency and the preferred dose is, what the latter would have been if marijuana of much higher potency were smoked. This is an important question since potencies of marijuana are increasing rapidly. At present, potencies of marijuana cultivated in The Netherlands may contain $5-15 \%$ THC. It is hard to imagine that subjects would consume as much of a cigarette containing a very high THC concentration as one containing a very low one. Future research determining marijuana users' preferred dose should also include marijuana with much higher potencies. It may then appear that the relationship between consumed THC dose 
and cigarette potency was attributable to the subjects' inability to discriminate among potencies spanning a relative narrow range. A broader range of cigarette potencies would probably allow regular marijuana users to discriminate well enough to alter their consumption and thereby come closer to administering the same preferred dose after every one.

On the basis of the laboratory study's results, the highest dose of THC administered in subsequent driving studies was defined as $300 \mu \mathrm{g} / \mathrm{kg}$; other doses were 100 and $200 \mu \mathrm{g} / \mathrm{kg}$, and placebo. Marijuana cigarettes were prepared from batches with potencies varying from $1.75 \%$ to $3.58 \%$, the highest that were then available. If future research shows that current users of marijuana prefer much higher THC doses when they smoke very potent marijuana, the results of the current program can only serve as an indication of the minimal effects the drug may have on actual driving performance. If, on the other hand, it shows that users prefer a THC dose of about $300 \mu \mathrm{g} / \mathrm{kg}$ to reach their desired 'high' irrespective of the potency of the drug, the results presented in this report are truly valid estimates of what may happen in daily life after smoking THC doses that induce a preferred 'high'.

\subsection{The Driving Tests}

Three different tests were applied in the experiments that progressively increased in the number of skills employed by the driver: road tracking, car following and city driving. The first is now a standardized test which, in more than 40 studies, has proven to be very sensitive to sedation produced by a variety of medicinal and social drugs, including alcohol. The car following and city driving test were developed following the recognition that parameters measured in the standard test fail to represent all abilities considered important for safe driving. This section will describe the differences between the driving tests and the relevance of each to traffic safety.

The difference between the three driving tests can be characterized in many ways. The one described here is that in terms of the type of information processing each requires. Two distinct types of human information processing can be distinguished (Shiffrin and Schneider, 1977): 'automatic' and 'controlled'. The former is capable of accepting an enormous volume of perceptual information at a relatively high rate to mediate coordinated multi-effector responding in a normally stable input-output relationship. This process is not generally under voluntary control, proceeds in parallel with the stream of consciousness and involves no decision making. Controlled information processing is much slower but highly adaptive. It begins with the conscious perception of a discrete event or situation. Identification of the meaning of that perception is made by comparing it with information stored in memory. A decision involving the 
selection and execution of a particular series of motor responses and/or the suppression of a motor routine in progress occurs next. This process can proceed in parallel with an automatic process and the integrity of both are essential for safe driving.

Aspects of both types of information processing are present in most well practiced tasks. Most would agree that skilled performance is the integrated sum of automatic and controlled information processing and that their respective weights vary constantly with the task requirements. The more controlled information processing a task requires, the more it is experienced as demanding effort. According to this concept, lateral position control in the road tracking test depends principally upon automatic information processing. The car following test requires somewhat more controlled information processing and driving in urban traffic, even more. The concept can be illustrated by the ease of having a conversation with other occupants of a vehicle while driving. It is easy to converse with them while driving on a highway in light traffic. It becomes somewhat more difficult when driving in heavy traffic and maintaining a safe headway behind cars travelling in a platoon. Conversing while driving in urban traffic is even more difficult and, at times, even impossible for most drivers. Thus, the more controlled processing involved in a particular situation, the more effort the driving task demands and the less 'spare capacity' is left for having a conversation with other occupants. This concept of effort relates to task or computational demands, and often, to the allocation of attention. There exists, however, also another concept of effort which relates to brain arousal mechanisms (Kahneman, 1973; Pribram and McGuinness, 1975) or the required psychological state (Hockey, 1986). If one perceives a discrepancy between his actual state of arousal and that required to efficiently accomplish the task, he will first attempt to resolve the difference by compensatory effort, e.g. focussing attention. Failing that, he will try to reduce task demands to what can be efficiently accomplished in the deficient arousal state. The latter can be accomplished during driving by reducing speed or assuming a greater headway to compensate for slower reaction times.

It is sensible to keep the relevance of the separate driving tests for driving and traffic safety in mind while discussing THC's effects on driving performance. It is obvious that the road tracking test measures only a few aspects of driving behavior. Yet proper road tracking is a general prerequisite for safe driving. Consequently, drugs that substantially impair a driver's fundamental road tracking ability possess a real potential for adversely affecting driving safety. Whether this means that drivers under the influence of such drugs have also a greater probability of becoming involved in a traffic accident seems plausible but has yet to be determined. The same kind of reasoning applies to the car following test. It measures only a few additional aspects of driving behavior, but their impairment is also incompatible with safe driving. The city driving test 
measures most aspects of driving behavior and therefore comes closest to reality. Driving in urban traffic is so common in daily life that any drug-induced impairment found with this test should be considered as the most important, though not necessarily the earliest, sign that the drug possesses properties that adversely affect traffic safety.

\subsection{Effects of THC on Driving Performance}

\subsubsection{Results of Present Studies}

One of the issues addressed by the first driving study was whether it would be safe to continue using the same approach for subsequent on-road studies in traffic. The first group complied with all instructions, even after high doses of THC. Changes in mood were often reported but the subjects were always able to complete every ride without major interventions by the driving instructors and their safety was never compromised. The same occurred in the subsequent studies showing that it is possible to safely study marijuana's effects on actual driving performance in the presence of other traffic. In this respect, the drug is no different from many others studied by the same investigators and their colleagues.

The standard test measured the subjects' ability to maintain a constant speed and a steady lateral position between the lane boundaries. Standard deviation of lateral position, SDLP, increased after marijuana smoking in a dose-related manner. The lowest dose, i.e. $100 \mu \mathrm{g} / \mathrm{kg}$ THC, produced a slight elevation in mean SDLP, albeit significant in the first driving study. The intermediate dose, i.e. $200 \mu \mathrm{g} / \mathrm{kg} \mathrm{THC}$, increased SDLP moderately; and, the highest, i.e. $300 \mu \mathrm{g} / \mathrm{kg}$ THC, substantially. It is remarkable how well the changes in SDLP following THC in the first driving study were replicated in the second, in spite of the many differences in the ways they were designed. The replication of THC's effects on SDLP substantiates the generality of these results. Other objective measures obtained by this test were much less affected by THC. Mean speed was somewhat reduced following the higher THC doses, but the effects were relatively small (max. 1.1 $\mathrm{km} / \mathrm{h}$ or $0.7 \mathrm{mph}$ ). Standard deviations of speed and steering wheel movements were unaffected by the drug. Subjective ratings of perceived driving quality followed a similar pattern as SDLP indicating that the subjects were well aware of their diminished ability to control the vehicle after marijuana smoking.

The car following test measured the subjects' ability to follow a leading car with varying speed at a constant distance. All THC doses increased mean headway, but according to an inverse dose-response relationship. This type of relationship was unexpected and probably due to the particular design of the second driving study, i.e. the ascending dose series. It means that subjects were very cautious the first time they undertook the test under the influence of THC 
(i.e. after the lowest dose) and progressively less thereafter. As a consequence of this phenomenon, mean reaction time to changes in the preceding car's speed also followed an inverse dose-response relationship. Statistical adjustment for this confounding by analysis of covariance indicated that reaction times would not have increased significantly if the mean headway were constant. Coefficient of headway variation increased slightly following THC. Together, these data indicate that there is no more than a slight tendency towards impairment in car following performance after marijuana smoking. They also show that subjects try to compensate for anticipated adverse effects of the drug by increasing headway, especially when they are uncertain of what these might be. As in the standard test, subjects' ratings of driving quality corresponded to the objective changes in their performance.

The city driving study measured the subjects' ability to operate a vehicle in urban traffic. For reasons mentioned in the respective chapter the THC dose in that study was restricted to $100 \mu \mathrm{g} / \mathrm{kg}$. For comparative purposes another group of subjects was treated with a modest dose of alcohol, producing a mean BAC of about $0.04 \mathrm{~g} \%$. Results of the study showed that the modest dose of alcohol, but not THC, produced a significant impairment in driving performance, relative to placebo. Alcohol impaired driving performance but subjects did not perceive it. THC did not impair driving performance yet the subjects thought it had. After alcohol, there was a tendency towards faster driving and after THC, slower.

The results of these studies corroborate those of previous driving simulator and closed-course tests by indicating that THC in single inhaled doses up to $300 \mu \mathrm{g} / \mathrm{kg}$ has significant, yet not dramatic, dose-related impairing effects on driving performance. They contrast with results from many laboratory tests, reviewed by Moskowitz (1985), which show that even low doses of THC impair skills deemed important for driving, such as perception, coordination, tracking and vigilance. The present studies also demonstrated that marijuana can have greater effects in laboratory than driving tests. The last study, for example, showed a highly significant effect of THC on hand unsteadiness but not on driving in urban traffic.

\subsubsection{Drug Plasma Concentrations and Driving Performance}

One of the program's objectives was to determine whether it is possible to predict driving impairment by plasma concentrations of THC and/or its metabolite, THC- $\mathrm{COOH}$, in single samples. The answer is very clear: it is not. Plasma of drivers showing substantial impairment in these studies contained both high and low THC concentrations; and, drivers with high plasma concentrations showed substantial, but also no impairment, or even some improvement. The first driving study showed that impairment in the road tracking test was nearly 
the same in the first and second test, executed between $40-60$ and 100-120 minutes after initiation of smoking, respectively. Plasma concentrations of THC and THC-COOH, however, were not the same during the tests: both were lower during the second than the first. The same pattern was found for ratings of perceived 'high'. It has been said that behavioral signs of intoxication, though small, outlast physiological and subjective reactions to THC (Reeve et al., 1983; Yesavage et al., 1985). To examine this hypothesis, future research should extend actual driving performance measurements to 4, 8, 16 and 24 hours after smoking. If driving impairment still occurs after THC disappears from plasma, it could mean that previous epidemiological research has underestimated the proportion of drivers who were driving under the influence of cannabis at the times their accidents occurred.

Mean speed was the only measure of driving performance that was even moderately related to plasma concentrations of the drug. Subjects with higher THC concentrations in plasma drove slower in the standard road tracking test (correlations varying from $r=-.18$ to $r=-.72$ between conditions). This effect might have been even more pronounced if the subjects had not been instructed to drive at a particular speed, and if they had had no feedback from the speedometer.

\subsubsection{Cannabis versus Alcohol and Other Psychotropic Drugs}

How do marijuana's effects on driving performance compare to those of alcohol? There are two sources from which one can draw to answer the question. Information can be directly obtained from studies comparing THC and alcohol effects in the same experiment; and, indirectly, from studies wherein alcohol's effects were assessed using the same methods as applied in the present THC studies. As mentioned in Chapter 3, most closed-course studies on THC also measured alcohol's effects (BACs between 0.04 and $0.10 \mathrm{~g} \%$ ). It was generally concluded that THC's effects were less than alcohol's, especially at BACS above $0.08 \mathrm{~g} \%$. The city driving study in the present program also compared the effects of modest doses of alcohol and THC. For doses administered in that study, alcohol produced the greater effects. Indirect evidence concerning the relative effects of THC and alcohol can be obtained from three studies. First, the alcohol calibration study by Louwerens et al. $(1985,1987)$ which resembled our first driving study in many respects. According to their empirical equation, THC's effects on SDLP were equal to or less than that of $B A C=0.07 \mathrm{~g} \%$. More recently, studies by Riedel et al. (1989) and Ramaekers et al. (1992a) measured the effects of low doses of alcohol (BACs of 0.05 and $0.03 \mathrm{~g} \%$ respectively) on SDLP. Both groups applied the standard test in the presence of other traffic, as in our second driving study, but on another highway. Mean SDLPs were respectively about 5.0 
and $2.5 \mathrm{~cm}$ higher while driving after alcohol than placebo. The former elevation is greater than that produced by the highest THC dose in our study. The latter lies between the effects of 200 and $300 \mu \mathrm{g} / \mathrm{kg}$ doses, which were 1.8 and $2.9 \mathrm{~cm}$ respectively. There was some discrepancy between alcohol's effects on SDLP in the more recent studies and those predicted by the empirical equation: the former were higher than predicted. The discrepancy appears to be related to the difference between alcohol's effects on the ascending and descending phases of its pharmacokinetic profile. Louwerens measured alcohol's effects at the time when $\mathrm{BAC}$ was at the ascending but Riedel and Ramaekers measured them during the descending phase. Notwithstanding methodological differences among studies, both direct and indirect evidence converge on the conclusion that THC's effects after doses up to $300 \mu \mathrm{g} / \mathrm{kg}$ never exceed alcohol's at BACs of $0.08 \mathrm{~g} \%$.

How do marijuana's effects on driving performance compare to those of drugs other than alcohol? No direct comparisons have ever been made, but many studies employing the standard road tracking test were conducted for measuring other drugs' effects on SDLP during the last decade. The results from a few will be mentioned. Note that THC's greatests effects on SDLP were 3.7 and $2.9 \mathrm{~cm}$ in our first and second driving study, respectively. Diazepam (Valium) given for one week in a low therapeutic dose ( $5 \mathrm{mg}$, thrice daily) caused anxious patients to drive with a mean SDLP about $7 \mathrm{~cm}$ higher than their premedication baseline (Van Laar et al., 1992). The same drug and dose given over the same period caused healthy volunteers to drive with a mean SDLP about $6 \mathrm{~cm}$ higher than placebo (Van Veggel and O'Hanlon, 1993). Lorazepam (Ativan'), another anxiolytic, given twice daily for one week in a $1.5 \mathrm{mg}$ dose to healthy volunteers (Volkerts et al., 1988) and a $2 \mathrm{mg}$ dose to patients (Vermeeren et al., 1993), produced an elevation of SDLP of about $10 \mathrm{~cm}$ in both cases. Amitriptyline (Elavil"), a widely prescribed antidepressant, given in a dose of $50 \mathrm{mg}$ at night and $25 \mathrm{mg}$ in the morning caused healthy volunteers to drive with a mean SDLP about $6 \mathrm{~cm}$ higher than placebo (Robbe et al., 1989). Flurazepam (Dalmane), a hypnotic, was administered to insomniacs and its 'hang-over' effects on SDLP were measured 10-11 hours after ingestion. A $15 \mathrm{mg}$ dose of flurazepam elevated mean SDLP by about $4 \mathrm{~cm}$; a $30 \mathrm{mg}$ dose, $7 \mathrm{~cm}$. Antihistamines also cause sedation and, consequently, impair road tracking performance. Triprolidine (Actifed") increased SDLP by $3.5 \mathrm{~cm}$ after a single $5 \mathrm{mg}$ dose (Riedel et al., 1990); and, diphenhydramine $50 \mathrm{mg}$ (Benadryl Kapseals) increased SDLP by $4.5 \mathrm{~cm}$ (Ramaekers et al., 1992b). This is not to say that all psychotropic drugs produce greater elevations of SDLP than THC. Many in the same and other experiments had less effect than THC did in our studies. These examples are merely cited to indicate that THC's effects as measured in the standard test were in no way unusual. In so far as its effects on SDLP are concerned, THC was just another moderately impairing drug. 
The foregoing comparisons might be misleading. THC's effects differ qualitatively from many other drugs, especially alcohol. For example, subjects drive faster after drinking alcohol and slower after smoking marijuana (Hansteen et al., 1976; Casswell, 1979; Peck et al., 1986; Smiley et al., 1987). Moreover, the simulator study by Ellingstad et al. (1973) showed that subjects under the influence of marijuana were less likely to engage in overtaking maneuvers, whereas those under the influence of alcohol showed the opposite tendency. Very importantly, our city driving study showed that drivers who drank alcohol overestimated their performance quality whereas those who smoked marijuana underestimated it. Perhaps as a consequence, the former invested no special effort for accomplishing the task whereas the latter did, and successfully. This evidence strongly suggests that alcohol encourages risky driving whereas THC encourages greater caution, at least in experiments. Another way THC seems to differ qualitatively from many other drugs is that the former's users seem better able to compensate for its adverse effects while driving under the influence. Weil et al. (1968) were among the earliest authors who mentioned the possibility that marijuana users can actively suppress the drug's adverse effects. They presumed that THC's effects were confined to higher cortical functions without any general stimulatory or depressive effect on lower brain centers. According to them, the relative absence of neurological, as opposed to psychiatric, symptoms in marijuana intoxication suggests this possibility. More recently, Moskowitz (1985) concluded that the variety of impairments found after marijuana smoking could not be explained by decrements in sensory or motor functions which led him to hypothesize that some important central cognitive process is impaired by THC, without saying what it is. The recent discovery of abundant cannabinoid receptor concentrations in the cerebral cortex and the hippocampal formation corroborates these hypotheses and, with other findings to come, will certainly greatly enhance our understanding of the drug's psychopharmacological effects.

\subsubsection{Why are THC's Effects on Driving Performance relatively Small?}

It is a natural question why the effects of marijuana on actual driving performance appear to be so small. As in many previous investigations, subjects attempted to compensate for anticipated adverse effects of marijuana smoking. Our subjects were aware of the impairing effects of THC as shown by lower ratings of perceived driving quality. Consequently, they invested more effort to accomplish the driving tests following THC than placebo. Furthermore, in the car following test, they drove at a greater headway after marijuana smoking; and, in both road tracking and city driving tests, they slightly reduced their driving speed. Yet despite their effort, subjects were unable to fully compensate for 
THC's adverse effects on lateral position variability. This is because SDLP is primarily controlled by an automatic information processing system which operates outside of conscious control. The process is relatively impervious to environmental changes, as shown by the high reliability of SDLP under repeated placebo conditions, but highly vulnerable to internal factors that retard the flow of information through the system. THC and many other drugs are among these factors. When they interfere with the process that restricts SDLP, there is little the afflicted individual can do by way of compensation to restore the situation. Car following and, to a greater extent, city driving performance depend more on controlled information processing and are therefore more accessible for compensatory mechanisms that reduce the decrements or abolish them entirely.

This still leaves the question open why performance appears to be more affected by THC in laboratory than actual driving tests. Many researchers defend the primacy of laboratory performance tests for measuring drug effects on skills related to driving on the basis of superior experimental control. Certainly some control is always necessary to reduce the confounding influence of extraneous factors that would otherwise so increase measurement error as to totally obscure the drug's effects. However, only some extraneous factors are truly sources of measurement error and others either attenuate or amplify drug effects in real driving and must be considered as relevant to a test's predictive validity. Simply eliminating all of them, first, removes their normal mediating influence on the drug effect, and secondly, affects the subject's motivation to perform the test by making it appear 'unreal'. Controlling the test usually involves drastic simplification and restriction of response options. The desire in doing this is to isolate a particular driving skill and determine how it changes under the influence of drugs. However, drivers always apply numerous skills in parallel and series. Should one become deficient, they are often able to compensate in a number of ways to achieve a satisfactory level of proficiency. Thus the demonstration of some particular skill decrement in the laboratory in no way indicates that this would ultimately reduce driving safety in reality. Finally there are some skills that simply can not be measured in laboratory tests, at least not easily enough to make it a routine matter. The acquisition of any skill which depends upon automatic information processing requires practice over weeks or months. After learning to drive, subjects possess such skills in abundance and one can only demonstrate how they vary with drug effects in the real task or a very close approximation thereof.

Profound drug impairment constituting an obvious traffic safety hazard could as easily be demonstrated in a laboratory performance test as anywhere else. But THC is not a profoundly impairing drug. It does affect automatic information processing, even after low doses, but not to any great extent after high doses. It apparently affects controlled information processing in a variety of laboratory tests, but not to the extent which is beyond the individual's ability to control 
when he is motivated and permitted to do so in real driving. In short, it would appear as if over-control in laboratory performance tests has resulted in a misimpression of THC's effect, incomplete in some respects and exaggerated in others. The actual driving tests may provide a more realistic impression of the drug's effects, albeit still incomplete and perhaps tending to minimize them with respect to more complex driving situations that come closer to 'worst case'.

The degree of experimental control also varied between driving tests in this series in ways affecting the subjects' motivation. This is illustrated by a comparison between the first and second driving study. The standard road tracking test was applied in both, first in the absence and then in the presence of other traffic. It was only during the former that disturbing observations of two individuals' attentional deficits caused the driving instructor to intervene. Driving in the presence of other traffic, subjects were always able to complete the rides without intervention. Lateral position control, an automatic process, did not change as a consequence of the absence or presence of other traffic. What did change was the subjects' motivation to focus attention, a controlled process. Motivation in the second study was very probably affected by recognition of the increased risk of the untoward consequences of wandering attention. This means that the intrinsic motivation produced by the reality of the test situation is an important mediator of THC's effects on performance.

Compensatory mechanisms help the driver under the influence of marijuana to maintain an effective level of performance but with an associated cost. If drivers compensate for THC's adverse effects by diminishing driving demands (e.g. by reducing speed and/or increasing headway), this will occur without a reduction in spare capacity. But if they increase effort as well (e.g. by focusing attention), it will occur at the expense of spare capacity. Less capacity would be left for simultaneously performing another task, such as conversing with passengers, using a car telephone, or handling emergency situations. The information processing capacity these situations demand may well go beyond the driver's spare capacity with the result of impaired and perhaps dangerous driving. Results of the present program show that THC increases the mental load of driving, as shown by increased effort ratings and reduced heart rate variability, and consequently reduces spare capacity. This corroborates results from previous simulator and closed-course studies that with reasonable consistency show an adverse THC effect on subsidiary task performance (Smiley, 1986). Further research is required to determine marijuana's effects on actual driving performance when the driver is simultaneously performing another task or suddenly confronted with a situation that requires a rapid adaptive response. The latter was occasionally encountered during the city driving test, but only after a low THC dose. The city driving test should therefore be repeated with subjects consuming higher THC doses. 
Hazardous driving can also occur in situations that demand very little of the driver's information processing capacity. If the driving task is very monotonous and the demand is low, wandering attention may result in negligent monitoring with disastrous results. This is in fact what happened twice during the driving study on the closed road. After the highest THC dose, one subject failed to shift attention from the prescribed task to an unexpected event (screwdriver on the road); another failed to anticipate a normal event (end of circuit). Though even sober experienced drivers may experience similar deficits, the fact that it happened twice after the highest THC dose, and never after a lower dose or placebo, strongly suggests that drivers under the influence of THC would be unusually susceptible to attentional deficits during prolonged and monotonous driving.

\subsubsection{Concluding Remarks}

Epidemiological research has shown that THC is infrequently detected in the blood of fatally injured drivers as the only drug present. In most cases alcohol is also detected. The effects of the combination of THC and alcohol on actual driving performance have never been studied in the presence of other traffic. Closed-course studies have shown that the effects of both drugs, when taken in combination, are generally additive (Attwood et al., 1981; Peck et al., 1986). This may only be so for those behaviors that are similarly affected by both drugs given separately. Closer examination of the combined use is warranted in those driving situations where both drugs produce qualitatively different effects. It may well be so that alcohol reduces drivers' insight or motivation to the point where they would no longer attempt to compensate for the THC effect. As a result, the combined effects on drivers' performance could well be greater than the sum of either drug acting separately. There is therefore a great need for further research on marijuana and actual driving research, but now extended to the combination of marijuana and alcohol.

In summary, this program of research has shown that marijuana, when taken alone, produces a moderate degree of driving impairment which is related to the consumed THC dose. The impairment manifests itself mainly in the ability to maintain a steady lateral position on the road, but its magnitude is not exceptional in comparison with changes produced by many medicinal drugs and alcohol. Drivers under the influence of marijuana retain insight in their performance and will compensate where they can, for example, by slowing down or increasing effort. As a consequence, THC's adverse effects on driving performance appear relatively small. The methods used for demonstrating THC's influence on driving performance were the same as for showing driving impairment after other drugs. That is their strength and weakness: In so far as THC's effects resemble those of other psychoactive drugs, their effects in the same tests can be 
compared. To the extent that THC possesses other effects not shown in these tests, the chance increases that something of importance was overlooked. One can easily imagine situations where the influence of marijuana smoking might have an exceedingly dangerous effect; i.e., emergency situations which put high demands on the driver's information processing capacity, prolonged monotonous driving, and after THC has been taken with other drugs, especially alcohol. Because these possibilities are real, this dissertation should not be considered as the final word. It should, however, remain for a while as the point of departure for subsequent studies that will ultimately complete the picture of THC's effects on driving performance.

We therefore agree with Moskowitz' (1985) conclusion that "any situation in which safety both for self and others depends upon alertness and capability of control of man-machine interaction precludes the use of marihuana (p. 342)." However, the magnitude of marijuana's, relative to many other drugs', effects also justify Gieringer's (1988) conclusion that "marijuana impairment presents a real, but secondary, safety risk; and that alcohol is the leading drug-related accident risk factor (p. 100)." Of the many psychotropic drugs, licit and illicit, that are available and used by people who subsequently drive, cannabis may well be among the least harmful. Campaigns to discourage the use of cannabis by drivers are certainly warranted. But concentrating a campaign on cannabis alone may not be in proportion to the safety problem it causes. 


\subsection{Summary of Conclusions}

The major conclusions from the present program are summarized as follows:

- Current users of marijuana prefer THC doses of about $300 \mu \mathrm{g} / \mathrm{kg}$ to achieve their desired 'high'.

- It is possible to safely study the effects of marijuana on driving on highways or city streets in the presence of other traffic.

- Marijuana smoking impairs fundamental road tracking ability with the degree of impairment increasing as a function of the consumed THC dose.

- Marijuana smoking which delivers THC up to a $300 \mu \mathrm{g} / \mathrm{kg}$ dose slightly impairs the ability to maintain a constant headway while following another car.

- A low THC dose $(100 \mu \mathrm{g} / \mathrm{kg})$ does not impair driving ability in urban traffic to the same extent as a blood alcohol concentration (BAC) of $0.04 \mathrm{~g} \%$.

- Drivers under the influence of marijuana tend to over-estimate the adverse effects of the drug on their driving quality and compensate when they can; e.g. by increasing effort to accomplish the task, increasing headway or slowing down, or a combination of these.

- Drivers under the influence of alcohol tend to under-estimate the adverse effects of the drug on their driving quality and do not invest compensatory effort.

- The maximum road tracking impairment after the highest THC dose $(300$ $\mu \mathrm{g} / \mathrm{kg}$ ) was within a range of effects produced by many commonly used medicinal drugs and less than that associated with a blood alcohol concentration (BAC) of $0.08 \mathrm{~g} \%$ in previous studies employing the same test.

- It is not possible to conclude anything about a driver's impairment on the basis of his/her plasma concentrations of THC and THC-COOH determined in a single sample. 


\subsection{Summary of Recommendations}

The recommendations for future research are summarized as follows:

- Future studies on marijuana's effects on driving performance should include the THC dose that currents users of marijuana prefer to achieve their desired 'high', i.e. $300 \mu \mathrm{g} / \mathrm{kg}$.

- Subjects in future studies should be familiarized with the potency of the material providing THC before the beginning of tests designed to measure its effects on performance.

- Future research determining marijuana users' preferred dose should involve administering THC in different marijuana preparations that encompass a wide range of potencies.

- Further research is required for determining marijuana's effects on actual driving performance in the presence of other traffic when the driver is simultaneously performing another task or suddenly confronted with a situation that requires a rapid adaptive response.

- The city driving test should be repeated with subjects consuming THC doses over $100 \mu \mathrm{g} / \mathrm{kg}$.

- Further research on marijuana and actual driving research should be extended to include combinations of marijuana and alcohol.

- Future research should extend actual driving performance measurements to $4,8,16$ and 24 hours after smoking. 


\section{SUMMARY}

This dissertation concerns the effects of marijuana smoking on actual driving performance. It contains separate reviews pertaining to the general properties of cannabis' main constituent, $\Delta^{9}$-tetrahydrocannabinol (THC), and previous research pertaining to its effects on driving performance and traffic safety. It goes on to describe a research program comprised of one laboratory and three driving studies wherein dose-effect relationships were measured in actual driving situations that progressively approached reality. The major purpose was to determine how much THC affects driving performance as its dose increases and what the implications for traffic safety might be. A secondary purpose was to determine whether it is possible to correlate changes in driving performance with THC plasma concentrations or those of its major (inactive) metabolite, 11-nor- $\Delta^{9}$-THC9-carboxylic acid (THC-COOH). A variety of driving tests were employed, including: maitenance of a constant speed and lateral position during uninterrupted highway travel, following a leading car with varying speed on a highway, and city driving. The purpose of applying different tests was to determine whether similar changes in performance under the influence of THC occur in all, thereby indicating a general drug effect on driving ability.

Chapters 1 and 2 provide the rationale for the dissertation and a general overview of cannabis, respectively. In the latter, the history of cannabis use, its current usage prevalence, chemistry and pharmacology, acute and chronic effects, and current therapeutic applications are discussed.

Chapter 3 concentrates on the literature pertinent to the effects of cannabis on driving. Epidemiology shows that people drive after cannabis use and that drivers involved in accidents often show the drug's presence. The results are, however, inconclusive because of the high proportion of cases who also used alcohol and the lack of proper control groups. The extent cannabis contributes to traffic accident causality remains obscure. Results from driving simulator and closed-course tests show that THC in single inhaled doses up to about $250 \mu \mathrm{g} / \mathrm{kg}$ has relatively minor effects on driving performance, certainly less than blood alcohol concentrations (BACs) in the range of $0.08-0.10 \mathrm{~g} \%$. In contrast, laboratory studies have repeatedly shown performance impairment occurring after 
inhaled doses as low as $40 \mu \mathrm{g} / \mathrm{kg}$. It becomes large and persistent after 100-200 $\mu \mathrm{g} / \mathrm{kg}$ doses. Tracking, divided attention and vigilance test performances were particularly vulnerable to THC's effects.

Chapter 4 describes a program of one laboratory and three driving studies and certain procedures that were common to all. These were compliance with ethical and legal standards, subject recruiting, marijuana cigarettes and smoking procedures, screening for the presence of other illicit drugs and alcohol, blood sampling procedures and quantitative analyses. Subjects in all studies were recreational users of cannabis, i.e. smoking marijuana or hashish more than once a month but not daily. They were all healthy, between 21 and 40 years of age, had normal weight and binocular acuity, and were licensed to drive an automobile. Subjects were accompanied in every driving test by an licensed driving instructor. Redundant control system in the test vehicle was available for controlling the car if emergency situations should arise. Marijuana and placebo marijuana cigarettes were supplied by the U.S. National Institute on Drug Abuse.

Chapter 5 presents the results of the laboratory study. It was conducted in a hospital under strict medical supervision to identify THC doses that recreational marijuana users were likely to consume before driving. Twenty-four subjects, twelve males and twelve females, participated. They were allowed to smoke part or all of the THC content in three cigarettes until achieving the desired psychological effect. Cigarettes were smoked through a plastic holder in a manner determined by the subjects. The only requirement was to smoke continuously for a period not exceeding 15 minutes. When subjects voluntarily stopped smoking, cigarettes were carefully extinguished and retained for subsequent gravimetric estimation of THC consumed. Six subjects consumed one cigarette, thirteen smoked two and four smoked three. The average amount of THC consumed was $20.8 \mathrm{mg}$, after adjustment for body weight, $308 \mu \mathrm{g} / \mathrm{kg}$. There were no significant differences between males and females, and frequent and infrequent users with respect to the weight adjusted preferred dose. It was decided that the maximum dose for subsequent driving studies would be $300 \mu \mathrm{g} / \mathrm{kg}$. This is considerably higher than doses that have usually been administered to subjects in experimental studies (typically, $100-200 \mu \mathrm{g} / \mathrm{kg}$ THC).

The study provided the opportunity for obtaining valuable information about THC's pharmacokinetics and its pharmacodynamic effects after marijuana smoking. Blood samples were repeatedly taken for measuring plasma concentrations of THC and THC-COOH. The subjects repeatedly performed certain simple laboratory tests, estimated their levels of intoxication and indicated their willingness to drive under several specified conditions of urgency. Heart rate was measured at these times. The secondary purpose of the laboratory study was that of specifying relationships between [THC] and [ $\mathrm{THC}-\mathrm{COOH}$ ] with changes in the other physiological, performance or subjective variables. Other results from the laboratory study showed that perceived 'high' and heart rate are very sensitive 
measures of marijuana intoxication which confirms prior findings. Impairments in laboratory tests performance were found at the time of peak subjective feelings but generally, objective impairment dissipated more rapidly than the feelings themselves.

The first driving study, described in Chapter 6, was conducted on a highway closed to other traffic. One objective of the study was to determine whether it would be safe to repeat the study on a normal highway in the presence of other traffic. The second objective was to define the dose-effect relationship between inhaled THC dose and driving performance. The same twelve men and twelve women who participated in the laboratory study served again as the subjects. They were treated on separate occasions with THC doses of $0,100,200$, and $300 \mu \mathrm{g} / \mathrm{kg}$. Treatments were administered double-blind and in a counterbalanced order. On each occasion, subjects performed a road tracking test beginning 40 minutes after initiation of smoking and repeated one hour later. The test, developed and standardized by O'Hanlon et al. $(1982,1986)$, involved maintaining a constant speed at $90 \mathrm{~km} / \mathrm{h}(56 \mathrm{mph})$ and a steady lateral position between the delineated boundaries of the traffic lane. Subjects drove $22 \mathrm{~km}$ $(13.6 \mathrm{mi})$ on a primary highway and were accompanied by a licensed driving instructor. The latter was charged with responsibility for ensuring safety at all times and was able to intervene, if necessary, using redundant vehicular controls. The primary dependent variable was the standard deviation of lateral position (SDLP), which has been shown to be both highly reliable and very sensitive to the influence of sedative drugs and alcohol. Other dependent variables were mean speed, and standard deviation of speed and steering wheel angle. Blood samples were taken prior to each driving test; and, performance in critical tracking and hand steadiness tests, heart rate, and blood pressure were measured after its termination. Questionnaires were repeatedly administered to estimate the 'high' and other subjective feelings.

All subjects were willing and able to finish the driving tests without great difficulty. The study demonstrated that marijuana impairs driving performance as measured by an increase in SDLP; all three THC doses significantly affected SDLP relative to placebo. The driving performance decrement after smoking marijuana persisted almost undiminished for two hours after smoking while drug plasma concentrations, perceived 'high' and heart rate elevation had decreased. Marijuana's effects on SDLP were compared to those of alcohol obtained in a very similar study by Louwerens et al. $(1985,1987)$. It appeared that THC's effects on SDLP were equivalent to those associated with BACs in the range of 0.03-0.07 g\%. Other driving performance measures were not significantly affected by THC. Intersubject correlations between plasma concentrations of the drug and driving performance after every dose were essentially nil. Thus, driving impairment cannot be predicted by prevailing plasma concentrations of THC or THC-COOH. Driving impairment was also not related to performance in the laboratory tests. 
Both the observed degree of driving impairment, and what subjects said and did, indicated that normal safeguards would be sufficient for ensuring safety in further testing. Hence, the final conclusion was to repeat this study on a normal highway in the presence of other traffic.

The second driving study, described in Chapter 7, was conducted to come a step closer to driving reality than its predecessor. Driving tests were now conducted on a highway in the presence of other traffic. The major objective of this study was to confirm the relationship between inhaled THC dose and lateral position variability in the context of a standard road tracking test. A secondary objective was to measure performance in another actual driving test, i.e. car following. The third objective was to continue efforts to correlate plasma concentrations of $\mathrm{THC}$ and $\mathrm{THC}-\mathrm{COOH}$ with driving performance impairment as measured in both tests.

A new group of sixteen subjects, equally comprised of men and women, participated in this study. A conservative approach was chosen in designing the present study in order to satisfy the strictest safety requirements. That is, the study was conducted according to an ascending dose series design where both active drug and placebo conditions were administered, double-blind, at each of three THC dose levels. THC doses were the same as those used in the previous study, namely 100,200 , and $300 \mu \mathrm{g} / \mathrm{kg}$. Cigarettes appeared identical at each level of treatment conditions and were smoked through a plastic holder in a fashion determined by the subject within a time limit of 10 minutes. If any subject would have reacted in an unacceptable manner to a lower dose, he/she would not have been permitted to receive a higher dose.

Two subjects at a time commenced smoking. Thirty minutes after onset of smoking the subjects performed a battery of laboratory tests (tracking, hand steadiness and body sway), yielded a blood sample, and rated their 'high' and other subjective feelings. They were then transported to a primary highway were the driving tests were performed. Two instrumented vehicles were employed. The subjects performed the car following test on a $16 \mathrm{~km}(9.9 \mathrm{mi})$ segment of the highway for about twelve minutes. After conclusion of the car following test, both subjects then commenced the road tracking test in separate instrumented vehicles. The highway was the same as for the car following test. Subjects drove $64 \mathrm{~km}(40 \mathrm{mi})$ without stopping in about 50 minutes. At the conclusion of this test, both subjects participated again in the car following test. Subjects were then transported back to the laboratory where they rated subjective feelings, yielded a blood sample, and repeated the test battery. The subjects' heart rate was registered continuously during both driving tests.

The road tracking test was the same as in the previous study except for its duration and the presence of other traffic. Subjects were instructed to maintain a constant speed of $95 \mathrm{~km} / \mathrm{h}(59 \mathrm{mph})$ and a steady lateral position between lane boundaries in the right traffic lane. They were allowed to deviate from this only 
if it would become necessary to pass a slower vehicle in the same lane. Data from the standard test were analyzed to yield the same performance measures as in the previous study; i.e. SDLP, mean and standard deviation of speed, and standard deviation of steering wheel angle. The car following test measures drivers' ability to perceive changes in a preceding vehicle's speed and to react in a manner maintaining a constant headway. It began as the preceding and the following vehicle, respectively driven by one of the driving instructors and the subject, operated in tandem on the slower traffic lane while travelling at a speed of $100 \mathrm{~km} / \mathrm{h}(62 \mathrm{mph})$. The subject was instructed to maintain a $50 \mathrm{~m}$ (164 ft) headway however the preceding vehicle's speed might vary. After driving in this manner for about one minute, the operator of the preceding vehicle released the accelerator pedal allowing its speed to fall to $80 \mathrm{~km} / \mathrm{h}(50 \mathrm{mph})$. Immediately thereafter, the operator of the preceding vehicle accelerated to $100 \mathrm{~km} / \mathrm{h}$ $(62 \mathrm{mph})$. The duration of one deceleration and acceleration maneuver was approximately 50 seconds and six to eight, depending upon traffic density, were executed during one test. The subject's average reaction time to the movements of the leading vehicle, mean headway and coefficient of variation of headway during maneuvers were taken as the dependent variables from this.

All subjects were able to complete the series without suffering any untoward reaction while driving. Road tracking performance in the standard test was impaired in a dose-related manner by THC and confirmed the results obtained in the previous closed highway study. The $100 \mu \mathrm{g} / \mathrm{kg}$ dose produced a slight elevation in mean SDLP, albeit nearly significant. The $200 \mu \mathrm{g} / \mathrm{kg}$ dose produced a significant elevation, of dubious practical relevance. The $300 \mu \mathrm{g} / \mathrm{kg}$ dose produced a highly significant elevation which may be viewed as practically relevant but unexceptional in comparison with similarly measured effects of many medicinal drugs. Following marijuana smoking subjects drove with an average speed that was only slightly lower than after placebo and very close to the prescribed level.

In the car following test, subjects maintained a headway of $45-50 \mathrm{~m}$ (148$164 \mathrm{ft}$ ) while driving in the successive placebo conditions. They lengthened mean headway by 8,6 and $2 \mathrm{~m}(26.2,19.7$ and $6.6 \mathrm{ft})$ in the corresponding THC conditions after 100,200 and $300 \mu \mathrm{g} / \mathrm{kg}$, respectively. The initially large drugplacebo difference and its subsequent decline is a surprising result. Our explanation for this observation is that the subjects' caution was greatest the first time they undertook the test under the influence of THC and progressively less thereafter. Reaction time to changes in the preceding vehicle's speed increased following THC treatment, relative to placebo. The administered THC dose was inversely related to the change in reaction time, as it was to headway. However, increased reaction times were partly due to longer headway. Statistical adjustment for this confounding resulted in smaller and non-significant increases in reaction time following marijuana treatment, the greatest impairment $(0.32 \mathrm{~s})$ being 
observed in the first test following the lowest THC dose. Headway variability followed a similar pattern as mean headway and reaction time; the greatest impairment was found following the lowest dose.

An important practical objective of this study was to determine whether degrees of driving impairment can be accurately predicted from either measured concentrations of THC in plasma or performance measured in potential roadside 'sobriety' tests of tracking ability or hand and posture stability. The results, like many reported before, indicate that none of these measures accurately predicts changes in actual driving performance under the influence of THC.

The program then proceeded into the third driving study, presented in Chapter 8, which involved tests conducted in high-density urban traffic. There were logical and safety reasons for restricting the THC dose to $100 \mu \mathrm{g} / \mathrm{kg}$. It was given to a group of regular cannabis users, along with placebo. For comparative purposes another group of regular alcohol users were treated with a modest dose of their preferred recreational drug, and again placebo, before undertaking the same city driving test. Two groups of sixteen new subjects apiece, equally comprised of men and women, participated. Subjects in the alcohol group were regular users of alcohol but not marijuana. Both groups were treated on separate occasions with active drug and placebo. Active marijuana was administered to deliver $100 \mu \mathrm{g} / \mathrm{kg}$ THC. The driving test commenced 30 minutes after smoking. The alcohol dose was chosen to yield a BAC approaching $0.05 \mathrm{~g} \%$ when the driving test commenced 45 minutes after onset of drinking. Active drug and placebo conditions were administered double-blind and in a counterbalanced order in each group.

Driving tests were conducted in daylight over a constant $17.5 \mathrm{~km}(10.9 \mathrm{mi})$ route within the city limits of Maastricht. Subjects drove their placebo and active drug rides through heavy, medium and low density traffic on the same day of the week, and at the same time of day. Two scoring methods were employed in the present study. The first, 'molar' approach, required the driving instructor acting as the safety controller during the tests to retrospectively rate the driver's performance using a standard scale. The second, a more 'molecular' approach, involved the employment of a specially trained observer who applied simple and strict criteria for recording when the driver made or failed to make each in a series of observable responses at predetermined points along a chosen route. Immediately prior to and following the driving tests subjects performed hand steadiness and time perception tests, yielded a blood sample, and were administered the same subjective questionnaires used in the previous studies.

The study showed that a modest dose of alcohol ( $\mathrm{BAC}=0.04 \mathrm{~g} \%$ ) produced a significant impairment in city driving as measured by the molar approach, relative to placebo. More specifically, alcohol impaired vehicle handling and traffic maneuvers. Marijuana, administered in a dose of $100 \mu \mathrm{g} / \mathrm{kg} \mathrm{THC}$, on the other hand, did not significantly change mean driving performance as measured 
by this approach. Neither alcohol nor marijuana significantly affected driving performance measures obtained by the molecular approach indicating that it may be relatively insensitive to drug-induced changes.

Driving quality as rated by the subjects contrasted with observer ratings. Alcohol impaired driving performance according to the driving instructor but subjects did not perceive it; marijuana did not impair driving performance but the subjects themselves perceived their driving performance as such. Both groups reported about the same amount of effort in accomplishing the driving test following placebo. Yet only subjects in the marijuana group reported significantly higher levels of invested effort following the active drug. Thus, there was evidence that subjects in the marijuana group were not only aware of their intoxicated condition but were also attempting to compensate for it. These seem to be important findings. They support both the common belief that drivers become overconfident after drinking alcohol and investigators' suspicions that they become more cautious and self-critical after consuming low THC doses by smoking marijuana.

The laboratory performance tests also discriminated between the drugs' effects. Hand steadiness was impaired following THC and improved following alcohol, relative to placebo. The difference between the drugs' effects was significant, both before and after the driving test. Impairment after THC was about as much as that produced by the same dose in the previous study, indicating equivalent sensitivities of the present and previous groups. Production of time intervals was not affected by alcohol, but THC significantly shortened interval production, relative to placebo.

Drug plasma concentrations were neither related to absolute driving performance scores nor to the changes that occurred from placebo to drug conditions. With respect to THC, these results confirm the findings in previous studies. They are somewhat surprising for alcohol but may be due to the restricted range of ethanol concentrations in the plasma of different subjects.

Chapter 9 concludes the dissertation with a general discussion of the results of the program and ends with a list of conclusions and recommendations. It starts with a discussion of the THC dose which marijuana users actually prefer for achieving their desired 'high'. Several questions are raised and discussed, such as: how do people regulate their THC consumption, what role plays familiarization with the drug, and what would the preferred dose have been if marijuana of much higher potency were smoked. The discussion then continues with a description of the differences between the driving tests in terms of the type of information processing each requires, automatic $v$ controlled, and the relevance of each to traffic safety.

Attention is further focussed on the effects of THC on driving performance. The results of the studies corroborate those of previous driving simulator and closed-course tests by indicating that THC in single inhaled doses up to $300 \mu \mathrm{g} / \mathrm{kg}$ 
has significant, yet not dramatic, dose-related impairing effects on driving performance. Standard deviation of lateral position in the road tracking test was the most sensitive measure for revealing THC's adverse effects. This is because road tracking is primarily controlled by an automatic information processing system which operates outside of conscious control. The process is relatively impervious to environmental changes but highly vulnerable to internal factors that retard the flow of information through the system. THC and many other drugs are among these factors. When they interfere with the process that restricts SDLP, there is little the afflicted individual can do by way of compensation to restore the situation. Car following and, to a greater extent, city driving performance depend more on controlled information processing and are therefore more accessible for compensatory mechanisms that reduce the decrements or abolish them entirely. Another important conclusion of the present studies is that it is impossible to say anything about a driver's impairment on the basis of his/her plasma concentrations of THC and THC-COOH determined in a single sample.

Marijuana's effects on driving performance were compared to those of many other drugs. It was concluded that THC's effects after doses up to $300 \mu \mathrm{g} / \mathrm{kg}$ never exceed alcohol's at BACs of $0.08 \mathrm{~g} \%$; and, were in no way unusual compared to many medicinal drugs'. Yet THC's effects differ qualitatively from many other drugs, especially alcohol. Evidence from the present and previous studies strongly suggests that alcohol encourages risky driving whereas THC encourages greater caution, at least in experiments. Another way THC seems to differ qualitatively from many other drugs is that the former's users seem better able to compensate for its adverse effects while driving under the influence.

It appears that performance is more affected by THC in laboratory than actual driving tests. Several reasons that may account for the apparent discrepancy are discussed. First, laboratory tests are experimentally controlled by drastic simplification which may affect a subject's motivation to perform the test by making it appear 'unreal'. Secondly, the restriction of response options in laboratory performance tests leave fewer possibilities for compensation. Finally, after learning to drive, subjects possess such skills in abundance and one can only demonstrate how they vary with drug effects in the real task or a very close approximation thereof.

Still one can easily imagine situations where the influence of marijuana smoking might have an exceedingly dangerous effect; i.e., emergency situations which put high demands on the driver's information processing capacity, prolonged monotonous driving, and after THC has been taken with other drugs, especially alcohol. Because these possibilities are real, this dissertation should not be considered as the final word. It should, however, remain for a while as the point of departure for subsequent studies that will ultimately complete the picture of THC's effects on driving performance. 


\section{SAMENVATTING}

Dit proefschrift handelt over de effecten van marihuana op rijgedrag. Het begint met een algemene verhandeling over cannabis en haar belangrijkste bestanddeel, $\Delta^{9}$-tetrahydrocannabinol (THC). Vervolgens wordt een overzicht gegeven van eerder onderzoek naar de effecten van deze drug op rijgedrag en verkeersveiligheid. Daarna wordt het onderzoekprogramma besproken, waarin dosis-effect relaties werden gemeten in verkeerssituaties die steeds beter de werkelijkheid benaderden. De voornaamste doelstelling van het onderzoek was om te bepalen in welke mate verschillende doses THC het rijgedrag beïnvloeden, en wat de gevolgen voor de verkeersveiligheid zouden kunnen zijn. Een andere doelstelling was om na te gaan of het mogelijk is om veranderingen in rijgedrag te relateren aan bloedplasma concentraties van THC of de belangrijkste (inactieve) metaboliet, 11-nor- $\Delta^{9}$-THC-9-carboxyl zuur (THC-COOH). In de onderzoeken zijn verschillende soorten rijtaken gebruikt: één waarbij de bestuurder met een zo constant mogelijke snelheid en laterale positie op een snelweg rijdt; één waarbij de bestuurder een voorligger, wiens snelheid varieert, op een constante afstand dient te volgen; en tenslotte één waarbij de bestuurder in stadsverkeer rijdt. De reden om verschillende soorten rijtaken te gebruiken was om na te gaan of THC een algemeen dan wel specifiek effect op rijgedrag heeft.

In Hoofdstuk 1 wordt in het kort de 'raison d'être' van het proefschrift beschreven. Hoofdstuk 2 bevat een algemene verhandeling over cannabis waarin een overzicht gegeven wordt van achtereenvolgens de geschiedenis van cannabis, de cijfers omtrent het gebruik, de chemie en farmacologie, de acute en chronische effecten en tenslotte de medicinale toepassingen.

In Hoofdstuk 3 wordt een overzicht gegeven van eerder onderzoek naar de effecten van cannabis op rijgedrag. Uit epidemiologisch onderzoek blijkt dat de drug vaak gevonden wordt in het lichaam van bestuurders die betrokken zijn bij ongelukken. Maar omdat bij velen van hen ook alcohol werd gevonden en goede controle-groepen in deze onderzoeken meestal ontbraken, kunnen er (voorlopig) nog geen definitieve conclusies getrokken worden omtrent het oorzakelijke karakter van cannabis bij verkeersongelukken. Resultaten van experimentele studies, die zijn uitgevoerd in simulatoren en op afgesloten wegen, laten zien dat 
THC in doses tot ca. $250 \mu \mathrm{g} / \mathrm{kg}$ relatief weinig effect heeft op de rijprestatie, in ieder geval minder dan bloedalcoholconcentraties (BAC's) van 0,8 tot $1,0 \%$. In schril contrast hiermee staan de resultaten van laboratorium studies waaruit blijkt dat THC al tot een achteruitgang van de taakverrichting kan leiden bij doses van $40 \mu \mathrm{g} / \mathrm{kg}$. De effecten worden groter en langduriger bij doses van $100-200 \mu \mathrm{g} / \mathrm{kg}$. Met name oog-hand coördinatie, verdeelde aandacht en vigilantie bleken gevoelig voor de effecten van THC.

In Hoofdstuk 4 wordt een beschrijving gegeven van het onderzoekprogramma dat bestond uit één laboratorium en drie rijstudies. Vervolgens worden bepaalde procedures beschreven die in alle studies gevolgd zijn. Deze hebben betrekking op de naleving van ethische en wettelijke regels, de proefpersonen-werving, de marihuana sigaretten en het rook-protocol, de controle op de aanwezigheid van andere psychofarmaca en alcohol, en tenslotte de afname en analyse van het bloed. De proefpersonen in de studies waren 'recreatieve' gebruikers van cannabis, oftewel personen die vaker dan eens per maand maar niet dagelijks marihuana of hashish roken. Zij waren allen gezond, tussen de 21 en 40 jaar oud, in het bezit van een geldig rijbewijs, en hadden een normaal gewicht en binoculair gezichtsvermogen. De proefpersonen werden tijdens elke rijtest begeleid door een rijinstructeur, die de beschikking had over dubbele bediening om in geval van nood te kunnen ingrijpen. De marihuana en placebo sigaretten werden geleverd door het Amerikaanse National Institute on Drug Abuse.

In Hoofdstuk 5 worden de resultaten van de laboratorium studie beschreven. Het voornaamste doel van deze studie was om de dosis THC te bepalen die recreatieve gebruikers van cannabis gebruiken om het gewenste psychologische effect (de 'high') te bereiken. Vierentwintig proefpersonen, twaalf mannen en twaalf vrouwen, namen deel aan het onderzoek. Zij mochten in maximaal 15 minuten net zoveel marihuana roken als nodig was om het gewenste psychologische effect te bereiken. De proefpersonen rookten de sigaretten, die 2,57\% THC bevatten en waren voorzien van een plastic mondstukje, op hun eigen manier. $\mathrm{Na}$ het roken werden de sigaretten voorzichtig gedoofd en bewaard voor de latere gravimetrische bepaling van de geconsumeerde dosis THC. Zes proefpersonen rookten één sigaret, dertien rookten er twee en vier rookten er drie (één proefpersoon bleek niet te hebben geïnhaleerd; zijn gegevens werden niet gebruikt bij de gegevensanalyse). De gemiddelde THC consumptie was $20,8 \mathrm{mg}$, of, rekening houdend met het individuele lichaamsgewicht, $308 \mu \mathrm{g} / \mathrm{kg}$. Er werden geen significante verschillen gevonden tussen mannen en vrouwen of tussen frequente en infrequente rokers. Op basis van deze resultaten werd besloten dat de maximale THC dosis in de rijstudies $300 \mu \mathrm{g} / \mathrm{kg}$ zou zijn, wat aanzienlijk meer is dan wat tot nu toe in de meeste experimentele studies werd gebruikt (gewoonlijk $100-200 \mu \mathrm{g} / \mathrm{kg}$ ).

De studie bood tevens de mogelijkheid om waardevolle informatie omtrent de farmacokinetiek en -dynamiek van THC na het roken van marihuana te 
verkrijgen. Daarom werden herhaalde malen na het roken bloedmonsters afgenomen om later bloedplasma concentraties van $\mathrm{THC}$ en $\mathrm{THC}-\mathrm{COOH}$ te bepalen. Verder verrichtten de proefpersonen telkens enkele simpele laboratoriumtaakjes, schatten de grootte van het psychologische effect (de 'high') en gaven aan of ze bereid zouden zijn een auto te besturen onder verschillende, nader gespecificeerde, omstandigheden waarbij de mate van urgentie varieerde. Ook de hartslagfrequentie werd gemeten. Het doel van deze metingen was om de relatie te bepalen tussen [THC] en [THC-COOH] enerzijds, en de veranderingen in de andere variabelen anderzijds. De grootste effecten van marihuana werden gevonden op de subjectieve 'high' en de hartslagfrequentie, wat een bevestiging is van eerdere bevindingen. Er werd een achteruitgang in de takkverrichting gevonden ten tijde van de maximale subjectieve 'high', maar deze verslechtering verdween sneller dan de subjectieve gevoelens zelf.

De eerste rijstudie, beschreven in Hoofdstuk 6, werd uitgevoerd op een afgesloten deel van een snelweg. Eén van de doelen van deze studie was om te bepalen of het veilig zou zijn om de studie te herhalen op een 'normale' snelweg, in de aanwezigheid van ander verkeer. Een tweede doel was om de dosis-effect relatie te bepalen tussen de gerookte THC doses en de rijprestatie. Aan deze studie namen dezelfde mannen en vrouwen deel als aan de laboratoriumstudie. $\mathrm{Z}_{\mathrm{ij}}$ rookten in verschillende sessies marihuana sigaretten met THC doses van 0 (placebo), 100, 200 en $300 \mu \mathrm{g} / \mathrm{kg}$. Deze doses werden 'dubbel-blind' en in een gerandomiseerde volgorde gegeven. In elke sessie werd twee maal dezelfde rijtest uitgevoerd, resp. 30 en 90 minuten na het roken. In de rijtest, ontwikkeld en gestandaardiseerd door O'Hanlon et al. $(1982,1986)$, diende de proefpersoon met een snelheid van $90 \mathrm{~km} / \mathrm{u}$ zo recht mogelijk tussen de witte strepen op de rechter rijbaan te blijven rijden. De proefpersonen reden een afstand van $22 \mathrm{~km}$ op de afgesloten snelweg. Ze werden begeleid door een rijinstructeur, die verantwoordelijk was voor de veiligheid en indien nodig kon ingrijpen middels dubbele bediening. De belangrijkste afhankelijke variabele was de standaard deviatie van de laterale positie (SDLP), een zeer betrouwbare maat die zeer gevoelig is voor de effecten van sederende geneesmiddelen en alcohol. Andere afhankelijke variabelen waren de gemiddelde snelheid en de variaties in snelheid en stuurbewegingen. Voor aanvang van de rijtesten werden bloedmonsters afgenomen. Na de rijtesten werden een oog-hand coördinatie en een hand-stabiliteit test verricht, en werden de hartslagfrequentie en bloeddruk gemeten. De proefpersonen vulden ook herhaaldelijk vragenlijsten in om de subjectieve 'high' en andere gevoelens te schatten.

Alle proefpersonen waren bereid en in staat om de rijtesten zonder al te grote problemen uit te voeren. De resultaten van deze studie toonden aan dat marihuana de rijprestatie negatief beïnloedt: alle drie THC doses leidden tot een significante verhoging van de SDLP t.o.v. placebo. Deze achteruitgang in de rijprestatie bleef vrijwel onveranderd bestaan in de tweede test, terwijl op dat moment de 
drug plasma concentraties alweer waren gedaald en de subjectieve 'high' en de hartslag verhoging waren afgenomen. De effecten van marihuana op SDLP werden vergeleken met die van alcohol zoals die gevonden waren in een vergelijkbare studie van Louwerens et al. $(1985,1987)$. Het bleek dat de effecten van THC vergelijkbaar waren met die van BAC's tussen 0,3 en 0,7\%o. Andere variabelen van de rijtest werden niet significant beïnvloed door THC. Voor alle THC doses gold dat de intersubject correlaties tussen plasma concentraties van de drug en de variabelen van de rijtest nihil waren. De achteruitgang in de rijprestatie kan dus niet worden voorspeld aan de hand van de plasma concentraties van THC of THC-COOH. De achteruitgang in de rijprestatie was ook niet gerelateerd aan de verrichtingen in de laboratorium testen. Zowel de grootte van de gevonden effecten als de uitingen en gedragingen van de proefpersonen gaven aan dat de normale veiligheidsmaatregelen voldoende zouden zijn om de veiligheid in verder onderzoek te garanderen. Daarom luidde de laatste conclusie van dit onderzoek dat de studie herhaald kon worden op een normale snelweg, in de aanwezigheid van ander verkeer.

De tweede rijstudie, beschreven in Hoofdstuk 7, werd uitgevoerd om de werkelijkheid nog beter te benaderen dan in het voorgaande onderzoek. De rijtesten werden wederom uitgevoerd op een snelweg, maar nu in de aanwezigheid van het overige verkeer. De eerste doelstelling van deze studie was om de gevonden dosis-effect relatie tussen de geconsumeerde THC dosis en de SDLP te bevestigen in dezelfde gestandaardiseerde rijtest. De tweede doelstelling was om de effecten van THC op de rijprestatie te bepalen middels een tweede rijtest, waarin de proefpersonen een voorgaande auto dienden te volgen (de zgn. "car following' test). De derde doelstelling was om weer te kijken of er een relatie gevonden kan worden tussen plasma concentraties van THC en THC-COOH en de rijprestatie in beide rijtesten.

Een nieuwe groep van zestien proefpersonen, gelijkelijk verdeeld over beide geslachten, nam deel aan deze studie. Er werd voor een conservatieve benadering gekozen bij het ontwerpen van deze studie om aan de stringente eisen ten aanzien van de (verkeers)veiligheid te voldoen. Dit betekende dat de studie werd uitgevoerd volgens een placebo-gecontroleerde 'ascending dose series design' met dezelfde drie THC doses als in de vorige studie, nl. 100, 200 en $300 \mu \mathrm{g} / \mathrm{kg}$. Dit design hield in dat de proefpersonen in de eerste twee weken van de studie, het eerste 'niveau', 0 en $100 \mu \mathrm{g} / \mathrm{kg}$ THC kregen; daarna, op het tweede niveau, 0 en $200 \mu \mathrm{g} / \mathrm{kg}$; en op het derde niveau 0 en $300 \mu \mathrm{g} / \mathrm{kg}$. De placebo en 'echte' marihuana sigaretten werden, op elk niveau 'dubbel-blind' en in een gerandomiseerde volgorde gegeven. De identiek ogende sigaretten waren voorzien van een plastic mondstuk en dienden op een voor de proefpersoon normale manier binnen 10 minuten te worden opgerookt. Als een proefpersoon op een bepaald niveau op een onacceptabele manier zou reageren, dan zou hij/zij niet worden toegelaten tot het volgende niveau. 
Twee proefpersonen begonnen op hetzelfde tijdstip te roken. Dertig minuten later doorliepen zij een test-batterij (ter bepaling van de oog-hand coördinatie en de instabiliteit van hand en lichaam), vulden zij enkele vragenlijsten in met betrekking tot hun gemoedstoestand, en werd een bloedmonster afgenomen. Daarna werden ze naar de snelweg gebracht, alwaar de rijtesten werden uitgevoerd in twee identieke, geïnstrumenteerde auto's. De proefpersonen begonnen met de 'car following' test. Deze test werd uitgevoerd op een stuk snelweg ter lengte van $16 \mathrm{~km}$ en duurde ongeveer twaalf minuten. Nadat beide proefpersonen deze test hadden uitgevoerd, begonnen zij vrijwel tegelijk aan de standaard rijtest. Hiervoor werd dezelfde snelweg gebruikt. De proefpersonen legden nu, zonder te stoppen, een afstand af van $64 \mathrm{~km}$, wat ongeveer 50 minuten in beslag nam. $\mathrm{Na}$ afloop van deze test werd wederom de 'car following' test uitgevoerd. Tijdens beide rijtesten werd continu de hartslag van de proefpersoon geregistreerd. $\mathrm{Na}$ afloop van de rijtesten werden de proefpersonen weer naar het laboratorium vervoerd, alwaar dezelfde handelingen (vragenlijsten, testjes en bloedmonster) werden uitgevoerd als voor aanvang van de rijtesten.

De standaard rijtest was dezelfde als in de voorgaande studie, met uitzondering van de duur van de test en de aanwezigheid van ander verkeer. De proefpersonen werden geïnstrueerd om met een snelheid van $95 \mathrm{~km} / \mathrm{u}$ zo recht mogelijk tussen de witte lijnen op de rechter rijbaan te blijven rijden. Ze mochten alleen van deze instructie afwijken als het noodzakelijk was om een langzamer rijdende voorligger te passeren. De gemeten variabelen waren dezelfde als in de vorige studie: de standaard deviatie van de laterale positie, de gemiddelde snelheid en de variaties in snelheid en stuurbewegingen. De 'car following' test meet het vermogen van de bestuurder om veranderingen in de snelheid van de voorligger waar te nemen en daarop zodanig te reageren dat de afstand tussen beide auto's constant blijft. De test begon op het moment dat beide auto's met een snelheid van $100 \mathrm{~km} / \mathrm{u}$ op de rechter rijbaan als een 'tandem' achter elkaar reden. De voorste auto werd bestuurd door één van de rijinstructeurs, de achterste door de proefpersoon. De proefpersoon kreeg de opdracht om de voorligger op een afstand van 50 meter te blijven volgen, ongeacht diens snelheidsveranderingen. $\mathrm{Na}$ ongeveer een minuut met een snelheid van $100 \mathrm{~km} / \mathrm{u}$ gereden te hebben liet de bestuurder van de voorgaande auto het gaspedaal los om de snelheid tot $80 \mathrm{~km} / \mathrm{u}$ te laten zakken, mits de verkeerssituatie dit toeliet. Direct daarna accelereerde de bestuurder weer naar een snelheid van $100 \mathrm{~km} / \mathrm{u}$. Deze manoeuvre van de- en accelereren duurde ongeveer 50 seconden en werd, afhankelijk van de verkeersdrukte, zes tot acht maal herhaald. De afhankelijke variabelen van deze test waren: de gemiddelde reactietijd op de snelheidsveranderingen van de voorligger, de gemiddelde afstand en de variatie-coëfficiënt van de afstand.

Alle proefpersonen waren in staat om alle sessies, op elk niveau, te doorlopen zonder dat er zich verkeersonveilige situaties voordeden. THC verslechterde het koershouden op de weg tijdens de standaard test op een dosis-afhankelijke 
manier, hetgeen een bevestiging was van de resultaten in de vorige studie. De 100 $\mu \mathrm{g} / \mathrm{kg}$ dosis leidde tot een lichte, zij het niet-significante, verhoging van de SDLP. De $200 \mu \mathrm{g} / \mathrm{kg}$ dosis leidde wel tot een significante verhoging van de SDLP, maar de praktische relevantie van dat effect is niet erg groot. De $300 \mu \mathrm{g} / \mathrm{kg}$ dosis leidde tot een significante verhoging die ook praktisch relevant genoemd kan worden. De effecten zijn echter niet uitzonderlijk als men ze vergelijkt met die van vele psychotrope geneesmiddelen. Hoewel de proefpersonen na het roken van marihuana ietwat langzamer reden dan na placebo, lag de gemiddelde snelheid altijd dicht in de buurt van de voorgeschreven snelheid.

In de 'car following' test bedroeg de gemiddelde afstand tot de voorligger 4550 meter tijdens de achtereenvolgende placebo condities. De gemiddelde afstand nam toe met 8, 6 en 2 meter in de corresponderende THC condities, respectievelijk na 100, 200 en $300 \mu \mathrm{g} / \mathrm{kg}$ THC. Het aanvankelijk grote en daarna afnemende verschil tussen de THC en placebo condities is een verrassende bevinding. De meest waarschijnlijke verklaring hiervoor is dat de voorzichtigheid van de proefpersonen het grootst was toen zij deze test voor het eerst onder invloed van marihuana (de $100 \mu \mathrm{g} / \mathrm{kg}$ dosis) uitvoerden, en steeds minder in de daaropvolgende THC condities. De gemiddelde reactietijden op de veranderingen in de snelheid van de voorligger waren groter na het roken van 'echte' marihuana dan na placebo. Evenals met de gemiddelde afstand het geval was, was de verhoging van de reactietijd het grootst na de laagste, en het kleinst na de hoogste THC dosis. De langere reactietijden waren echter gedeeltelijk het gevolg van grotere afstanden (een vertraging van een voorligger is moeilijker waar te nemen bij een grotere afstand). Covariantie-analyse van de reactietijden met afstand als covariaat leidde tot kleinere en niet-significante verhogingen in de reactietijd na marihuana, waarbij de grootste vertraging in reactietijd $(0,32 \mathrm{~s})$ gevonden werd in de eerste rit na de laagste THC dosis. De variatie-coëfficiënt van de afstand vertoonde hetzelfde patroon als de andere variabelen: de grootste toename werd gevonden na de laagste THC dosis.

Een belangrijke praktische doelstelling van deze studie was om te bepalen of het mogelijk is de mate van achteruitgang van de rijprestatie nauwkeurig te voorspellen aan de hand van de bloedplasma concentratie van THC of aan de hand van de effecten op de laboratorium testen. Dit bleek echter niet het geval te zijn, hetgeen een bevestiging is van wat eerdere onderzoeken reeds aangaven.

In de derde rijstudie, beschreven in Hoofdstuk 8, werden de rijtesten uitgevoerd in stadsverkeer. Op basis van de resultaten van de voorafgaande studies en uit veiligheidsoverwegingen werd de THC dosis in deze studie beperkt tot 100 $\mu \mathrm{g} / \mathrm{kg}$. Deze dosis en een placebo werden gegeven aan een groep regelmatige gebruikers van cannabis. Ter vergelijking werd aan een andere groep proefpersonen een kleine dosis alcohol, en placebo alcohol, verstrekt voordat zij dezelfde rijtest in de stad uitvoerden. Twee groepen van elk zestien personen, gelijkelijk verdeeld over beide geslachten, namen deel aan deze studie. De proefpersonen in 
de alcohol groep waren regelmatige gebruikers van alcohol, en hadden nog nooit cannabis gebruikt. Beide groepen ontvingen eenmaal een placebo en eenmaal de actieve drug. De actieve marihuana bevatte $100 \mu \mathrm{g} / \mathrm{kg}$ THC, en de rijtest begon 30 minuten na aanvang van het roken. De alcohol dosis werd zodanig gekozen dat een BAC van maximaal $0,5 \%$ zou zijn bereikt bij aanvang van de rijtest, 45 minuten na aanvang van het drinken. De actieve drug en placebo werden in beide groepen 'dubbel-blind' en in een gerandomiseerde volgorde aan de proefpersonen gegeven.

De rijtesten werden 's middags uitgevoerd volgens een vaste route van $17,5 \mathrm{~km}$ door Maastricht (totale duur ca. 45 minuten). De proefpersonen reden zowel door rustige buitenwijken als door de drukke binnenstad, en beide malen op dezelfde dag van de week en op hetzelfde tijdstip van de dag. Er werden twee verschillende beoordelingsprocedures gebruikt. De eerste was een 'molaire' methode waarbij de rijinstructeur, die naast de proefpersoon zat en de route aangaf, na afloop van de rit de rijprestatie retrospectief beoordeelde middels een standaard 'checklist' (de Praktische Rijvaardigheidstest van de ANWB). De tweede was een 'moleculaire' methode waarbij een getrainde beoordelaar, die achterin de auto zat, op vantevoren vastgestelde punten van de route bepaalde aspecten van het rijgedrag diende te beoordelen an de hand van nauwkeurig omschreven criteria. Zowel voor als na de rit gaven de proefpersonen een bloedmonster en vulden zij dezelfde vragenlijsten in als in de vorige studies. Tevens werden hun hand-stabiliteit en tijd-perceptie gemeten.

Uit de resultaten van de 'molaire' benadering bleek dat een lage dosis alcohol $(\mathrm{BAC}=0,3 \%$ ) de rijvaardigheid in stadsverkeer, in vergelijking met placebo, significant vermindert. Alcohol leidde met name tot meer fouten in de categorieen 'voertuigbehandeling' en 'verkeershandelingen'. In tegenstelling tot alcohol leidde een kleine hoeveelheid marihuana, met een THC dosis van $100 \mu \mathrm{g} / \mathrm{kg}$, niet tot een significante verandering in de rijprestatie, zoals gemeten met de 'molaire' benadering. Noch alcohol noch marihuana had een effect op de rijprestatie-maten zoals die gemeten zijn met de 'moleculaire' benadering. Dit kan betekenen dat deze beoordelingsmethode relatief ongevoelig is voor veranderingen in rijgedrag ten gevolge van de inname van psychofarmaca.

Er bestond een opvallend verschil tussen de proefpersonen en de rijinstructeur ten aanzien van de retrospectieve beoordeling van de rijprestatie. Volgens de rijinstructeur verminderde de lage dosis alcohol de rijkwaliteit van de proefpersonen maar namen ze dit zelf niet waar; een kleine hoeveelheid marihuana leidde volgens de rijinstructeur niet tot een achteruitgang in de rijprestatie maar de proefpersonen dachten zelf van wel. Op de vraag hoeveel inspanning de uitvoering van de rijtest gekost had, antwoordden beide groepen op vrijwel gelijke wijze in de placebo conditie. Echter, alleen de proefpersonen in de marihuana groep rapporteerden meer inspanning in de actieve drug conditie. Dit is een aanwijzing dat de proefpersonen in de marihuana groep zich niet alleen bewust 
waren van het feit dat ze 'onder invloed' van THC verkeerden, maar hiervoor ook actief probeerden te compenseren. Deze bevindingen zijn niet onbelangrijk. $\mathrm{Ze}$ ondersteunen de algemene opvatting dat automobilisten overmoedig worden na het drinken van alcohol, en het vermoeden van onderzoekers dat automobilisten voorzichtiger en zelf-kritischer worden na het consumeren een kleine hoeveelheid marihuana.

De laboratorium testen discrimineerden ook tussen de effecten van beide drugs. De hand-stabiliteit nam, ten opzichte van placebo, af na THC en toe na alcohol. Het verschil tussen de effecten van beide drugs was zowel voor als na afloop van de rijtest significant. De afname in hand-stabiliteit na THC was vergelijkbaar met de afname na dezelfde dosis in de vorige studie, wat aangeeft dat de huidige groep proefpersonen vrijwel even gevoelig voor de effecten van THC was als de groep in de vorige studie. De tijd-perceptie van de proefpersonen, gemeten door het produceren van tijdsintervallen, werd niet beïnvloed door alcohol, maar THC leidde tot kortere intervallen t.o.v. placebo.

De drug plasma concentraties waren noch aan de absolute rijprestatie-scores noch aan de veranderingen die optraden van de placebo naar de drug conditie gerelateerd. Met betrekking tot THC bevestigen deze resultaten de bevindingen in de vorige studies. Met betrekking tot alcohol zijn deze resultaten enigszins verrassend, maar dit is waarschijnlijk toe te schrijven aan het beperkte bereik van de ethanol concentraties in het plasma van de verschillende proefpersonen.

In Hoofdstuk 9 wordt het proefschrift afgesloten met een algemene discussie van de resultaten van het onderzoekprogramma, met op het eind een overzicht van de belangrijkste conclusies en aanbevelingen. De discussie begint met een bespreking van de THC dosis die marihuana gebruikers verkiezen om de gewenste 'high' te bereiken. Verschillende vragen worden opgeworpen en besproken, zoals: hoe reguleren gebruikers hun THC consumptie, welke rol speelt daarbij het al dan niet bekend zijn met de sterkte van de drug, en wat zou de geconsumeerde dosis zijn geweest als er marihuana gerookt was met een veel grotere THC concentratie. De discussie wordt daarna vervolgd met een beschrijving van de verschillen tussen de rijtesten in termen van 'automatische' en 'gecontroleerde' informatieverwerkingsprocessen, en de relevantie van de testen met betrekking tot de verkeersveiligheid.

De rest van de discussie concentreert zich op de effecten van THC op rijgedrag. De resultaten van de in dit proefschrift beschreven studies bevestigen de bevindingen van eerdere studies in simulatoren en op afgesloten wegen, nl. dat THC, in doses tot $300 \mu \mathrm{g} / \mathrm{kg}$, leiden tot significante, nochtans niet dramatische, dosisafhankelijke effecten op het rijgedrag. De standaard deviatie van de laterale positie in de standaard test was de gevoeligste maat voor het aantonen van de negatieve effecten van THC. Dit komt doordat het koershouden op de weg voornamelijk een 'automatische' proces is dat zonder bewuste controle verloopt. Dit proces is relatief ongevoelig voor externe maar zeer gevoelig voor interne factoren die de 
informatieverwerking vertragen. Hiertoe behoren o.a. THC en vele andere stoffen. Wanneer deze stoffen interfereren met het min of meer 'automatische' proces van koershouden op de weg, gemeten middels de SDLP, dan kan de betreffende persoon weinig of niets via compenserende mechanismen doen om de situatie te herstellen. De volgtaak in de 'car following' test en het rijden in stadsverkeer zijn veel meer afhankelijk van gecontroleerde informatieverwerkingsprocessen. Deze zijn veel toegankelijker voor compenserende mechanismen om de achteruitgang in de rijprestatie te verminderen of zelfs geheel teniet te doen. Een zeer belangrijke conclusie van de rijstudies is, dat het niet mogelijk is om op basis van de THC en THC-COOH concentraties in één enkel bloedmonster iets te zeggen over de rijprestatie van de betreffende automobilist.

Marihuana's effecten op rijgedrag werden vergeleken met die van vele andere genees- en genotmiddelen. Er werd geconcludeerd dat THC's effecten, bij doses tot $300 \mu \mathrm{g} / \mathrm{kg}$, nooit groter waren dan die van alcohol bij een BAC van $0,8 \%$, en zeker niet uitzonderlijk waren in vergelijking met de effecten van vele geneesmiddelen. De verschillen tussen THC en andere stoffen zijn echter vooral kwalitatief van aard, vooral in vergelijking met alcohol. De bevindingen van deze en eerdere studies wekken de stellige indruk dat alcohol risicovoller rijgedrag stimuleert en THC tot meer voorzichtigheid leidt, tenminste in experimenteel onderzoek. Een ander kwalitatief verschil tussen THC en andere stoffen is dat personen onder invloed van THC beter in staat lijken te zijn om voor de nadelige effecten van deze drug op het rijgedrag te compenseren.

De negatieve effecten van THC blijken groter te zijn in het laboratorium, wanneer men zgn. 'driving related skills' test, dan 'op de weg'. Er worden verschillende redenen voor deze ogenschijnlijke discrepantie besproken. Ten eerste worden in het laboratorium vaak zeer eenvoudige vaardigheden getest, wat wellicht de motivatie van de proefpersonen negatief beïnvloed doordat de test niet realistisch overkomt. Ten tweede blijven er door de simplificatie van de test minder mogelijkheden tot compensatie over. En ten derde zijn personen die al enige jaren een rijbewijs hebben dermate geoefend in het autorijden dat eventuele effecten van drugs op deze vaardigheid alleen aangetoond kunnen worden in de 'echte' rijtaak of een goede benadering daarvan.

Toch kan men zich gemakkelijk situaties voorstellen waarin de invloed van marihuana een buitengewoon gevaarlijk effect zou kunnen hebben, zoals: noodsituaties die hoge eisen stellen aan de informatieverwerkingscapaciteit van de automobilist, langdurig rijden op een saaie (snel)weg, en wanneer marihuana gecombineerd wordt met een andere psychotrope stof, met name alcohol. Aangezien deze mogelijkheden niet denkbeeldig zijn, is met dit proefschrift het laatste woord over marihuana nog niet gezegd. Het zou voorlopig echter wel als uitgangspunt kunnen dienen voor toekomstige studies, die uiteindelijk het beeld van de effecten van marihuana op rijgedrag zullen moeten completeren. 


\section{RÉSUMÉ}

Dans ce mémoire nous traitons des effets de la consommation de marijuana sur les performances de conduite. Nous nous sommes tout d'abord attachés à étudier les différentes caractéristiques du $\Delta^{9}$-tetrahydrocannabinol (THC), qui est le principal constituant de la marijuana, puis nous rappelons les recherches qui ont déjà été faites concernant les effets de cette drogue sur les performances de conduite et sur la sécurité routière. Nous décrivons ensuite un programme de recherche qui comprend une étude en laboratoire et trois études en condition réelle de conduite. Ce programme de recherche a permis de déterminer les implications de l'effet de dose au cours de véritables essais de conduite se rapprochant progressivement de la réalité. En effet, l'objectif principal de cette recherche était de voir dans quelle mesure le THC affecte les performances de conduite lorsque la dose consommée augmente, et quelles conséquences cela peut avoir sur la sécurité routière. Nous avions un objectif secondaire qui était de savoir s'il existe une corrélation entre la variation des performances de conduite et la concentration plasmatique de THC ou son principal métabolite (inactif), l'acide-9-carboxylique-11-norbornyle- $\Delta^{2}$-THC(THC-COOH). Plusieurs types de tests de conduite ont été employés qui consistaient: à rouler sur autoroute sans interruption en maintenant une vitesse constante et une certaine position latérale; à suivre sur autoroute une autre voiture dont la vitesse varie; à rouler en zone urbaine. La variété de ces tests devait permettre de déterminer si le THC entraîne à chaque fois les mêmes variations de performances, ce qui indiquerait que cette drogue agit de façon globale sur la capacité de conduite.

Au Chapitre 1 nous exposons la raison d'être de ce mémoire, puis au Chapitre 2 nous donnons une présentation générale de la marijuana. Dans le second chapitre nous traitons de l'histoire de l'utilisation de la marijuana, de l'ampleur de sa consommation à l'heure actuelle, de ses caractéristiques chimiques et pharmacologiques, de ses effets aigus et chroniques, et de ses applications thérapeutiques à l'heure actuelle.

Le Chapitre 3 est consacré aux études qui ont déjà été publiées concernant les effets de la marijuana sur la conduite automobile. Ainsi, les études épidémiologiques nous apprennent que les gens prennent le volant même après avoir fumé de 
la marijuana, et que chez les conducteurs impliqués dans des accidents de la route on trouve souvent la présence de cette drogue. Cependant, les résultats ne sont pas concluants, car dans la plupart des cas les conducteurs ont aussi consommé de l'alcool; de plus les groupes témoin sont insuffisants. Si bien qu'on ne sait pas encore exactement quel rang occupe la marijuana dans les causes d'accident de la route. Les résultats obtenus sur simulateurs de conduite et lors de tests sur circuits fermés montrent que l'inhalation d'une dose de THC ne dépassant pas $250 \mu \mathrm{g} / \mathrm{kg}$ a un effet relativement mineur sur les performances de conduite, et en tout cas inférieur à celui d'un taux d'alcoolémie compris entre $0,8 \%$ et $1,0 \%$. En revanche, les études en laboratoire ont montré à maintes reprises que l'inhalation de doses aussi faibles que $40 \mu \mathrm{g} / \mathrm{kg}$ entraînait une diminution des performances, laquelle devient importante et persistante avec l'inhalation de doses allant de 100 à $200 \mu \mathrm{g} / \mathrm{kg}$. L'effet du THC se ressent particulièrement sur les performances dans le cas de tests portant sur le suivi d'une trajectoire, sur l'attention répartie et sur la vigilance.

Au Chapitre 4 nous décrivons le programme de recherche qui est composé d'une étude en laboratoire et de trois études en conditions réelles de conduite. Nous y exposons certains protocoles communs aux trois études: la conformité à l'éthique et aux normes légales, le recrutement des volontaires, la fabrication des cigarettes de marijuana et leur mode d'inhalation, le dépistage d'autres drogues illégales ou d'alcool, les prélèvements de sang et analyses quantitatives. Dans ces trois études les volontaires étaient des consommateurs occasionnels de marijuana, en ce sens qu'ils fumaient de la marijuana ou du hachich plus d'une fois par mois, mais non quotidiennement. Ils étaient tous en bonne santé, âgés de 21 à 40 ans, de poids moyen, possédaient une acuité visuelle normale des deux yeux, et ils étaient titulaires d'un permis de conduire. Lors des tests de conduite, les volontaires étaient accompagnés d'un moniteur de conduite agréé. La voiture était équipée d'un système de commandes redondant qui permettait d'éviter la perte de contrôle du véhicule en cas d'urgence. Les cigarettes de marijuana et les cigarettes placebo étaient fournies par l'U.S. National Institute on Drug Abuse.

Au Chapitre 5 nous présentons les conclusions de l'étude en laboratoire. Menée en milieu hospitalier sous surveillance médicale stricte, cette étude visait à déterminer les doses de THC que les fumeurs occasionnels de marijuana sont susceptibles d'avoir absorbé lorsqu'ils prennent le volant. Vingt-quatre volontaires, douze hommes et douze femmes, ont participé à cette étude. Ils avaient le droit de fumer au maximum trois cigarettes de THC, et devaient s'arrêter lorsqu'ils avaient atteint l'état psychologique désiré. Ils pouvaient fumer de la manière qu'ils souhaitaient, mais devaient utiliser un fume-cigarette. La seule contrainte était de fumer sans interruption pendant une durée n'excédant pas 15 minutes. Lorsque les volontaires décidaient d'arrêter de fumer, leurs cigarettes étaient soigneusement éteintes et conservées en vue d'une estimation ultérieure de la masse de THC consumé. Six des volontaires ont fumé une cigarette, treize 
autres en ont fumé deux, et quatre en ont fumé trois. La quantité moyenne de THC consommé a été de $20,8 \mathrm{mg}$, soit, rapportée au poids des volontaires, 308 $\mu \mathrm{g} / \mathrm{kg}$. Il n'y a pas eu de différence significative entre hommes et femmes, et les doses choisies par les fumeurs réguliers et occasionnels, une fois rapportées à leur poids, ont été à peu près équivalentes. Il a donc été décidé que pour les études en situation de conduite la dose maximale serait de $300 \mu \mathrm{g} / \mathrm{kg}$. Cette dose est bien supérieure à celles habituellement administrées lors d'études expérimentales (soit en général 100 à $200 \mu \mathrm{g} / \mathrm{kg}$ ).

Cette étude a permis d'obtenir des informations importantes sur la pharmacocynétique du THC et les effets pharmacodynamiques de la consommation de marijuana. Des prélèvements sanguins ont régulièrement été effectués afin de mesurer la concentration de THC et de THC-COOH dans le plasma. Les volontaires ont à maintes reprises procédé à certains tests de laboratoire simples, estimé leur niveau d'intoxication, et indiqué s'ils seraient prêts à prendre le volant en fonction de l'urgence de la situation. A chaque fois le rythme cardiaque a été mesuré. L'étude en laboratoire avait également pour but de préciser les relations entre [THC] et [THC-COOH] d'une part et la modification des autres variables physiologiques, subjectives et de performance d'autre part. Il résulte également de cette étude que la sensation de bien-être et le rythme cardiaque sont des indicateurs très sensibles de l'intoxication par la marijuana, ce qui vient confirmer les résultats déjà obtenus par le passé. On a constaté lors des tests en laboratoire que la diminution des performances coïncidait avec des sensations subjectives accrues. Cependant les diminutions objectives de performances s'effacent en général plus vite que les sensations elles-mêmes.

La première étude en conduite réelle, que nous décrivons dans le Chapitre 6, s'est déroulée sur une autoroute fermée à toute circulation. Un des objectifs de cette étude était de déterminer si cette étude pourrait être reproduite en toute sécurité sur une autoroute normale ouverte à la circulation. Le deuxième objectif était de déterminer la relation entre la dose de THC inhalée et les performances de conduite. Ce sont les douze hommes et douze femmes ayant participé à l'étude en laboratoire qui ont là aussi servi de volontaires. Ceux-ci ont reçu successivement des doses de THC de 0,100,200, et $300 \mu \mathrm{g} / \mathrm{kg}$. Les traitements ont été administrés en double aveugle et de façon alternée. Pour chaque dose, les volontaires ont subi un test de conduite, consistant à suivre une file de circulation 40 minutes après avoir commencé à fumer, et ils ont subi un second test une heure plus tard. Ce test, mis au point et normalisé par O'Hanlon et al. (1982, 1986), consiste à garder une vitesse constante de $90 \mathrm{~km} / \mathrm{h}$ en maintenant sa position latérale entre les limites de la voie tracées au sol. Les volontaires ont parcouru $22 \mathrm{~km}$ sur une autoroute principale, accompagnés par un moniteur de conduite agréé. Ce dernier était responsable à tout moment de la sécurité du véhicule et pouvait pour cela intervenir, si nécessaire, grâce aux doubles commandes. La principale variable dépendante était la déviation standard de la position 
latérale qui, l'expérience l'a montré, est à la fois extrêmement fiable et très sensible à l'influence des sédatifs et de l'alcool. Les autres variables observées comprenaient la vitesse moyenne, l'écart standard de vitesse et l'écart angulaire standard du volant. Avant chaque test on a effectué des prélèvements sanguins; après chaque test, on a mesuré les performances sur les parties difficiles du circuit, procédé à des tests de stabilité des mains, et mesuré le rythme cardiaque et la pression artérielle. A chaque fois des questionnaires ont été soumis aux volontaires afin d'apprécier le bien-être et autres sensations subjectives.

Tous les volontaires ont voulu et pu terminer les tests de conduite sans grande difficulté. L'étude a démontré que la marijuana réduit les performances de conduite si l'on en juge par l'augmentation de la déviation de la position latérale; les trois doses de THC ont affecté cette déviation de manière significative en comparaison avec les placebo. La diminution des performances de conduite est restée pratiquement stable au cours des deux heures suivant la consommation de la marijuana, alors que les concentrations plasmatiques, la sensation de bien-être et l'accélération du rythme cardiaque avaient diminué. Les effets de la marijuana sur la déviation de la position latérale ont été comparés aux résultats obtenus concernant les effets de l'alcool dans une étude similaire menée par Louwerens et al. $(1985,1987)$. Il s'est avéré que les effets du THC sur la déviation latérale étaient équivalents aux effets provoqués par des taux d'alcoolémie compris entre $0,3 \%$ et $0,7 \%$. Les autres indicateurs des performances de conduite n'ont pas été affectés de façon significative par la consommation de THC. Pour chaque dose les corrélations interpersonnelles entre les concentrations de drogue dans le plasma et les performances de conduite n'ont pratiquement pas été significatives. On ne peut donc pas prévoir quelle sera la diminution des performances de conduite à partir des concentrations plasmatiques en THC ou THC-COOH mesurées avant de prendre le volant. La diminution des performances ne correspondait pas non plus aux résultats obtenus au cours des tests en laboratoire. Aussi bien le degré de la diminution des performances de conduite que les actes et déclarations des volontaires semblent indiquer que les mesures de sécurité habituelles devraient suffir pour les expérimentations futures. On peut donc conclure qu'il est possible de reproduire cette étude sur une autoroute normale ouverte à la circulation.

La seconde étude en conduite réelle, décrite dans le Chapitre 7, avait pour but de se rapprocher encore davantage des conditions réelles. Les tests de conduite ont donc été effectués sur une autoroute ouverte à la circulation. Le principal objectif de cette étude était de confirmer la relation existant entre la dose de THC inhalé et la variabilité de la position latérale dans le cas d'un test standard de suivi de trajectoire. Le deuxième objectif était de mesurer la performance de conduite à l'aide d'un autre test de conduite réelle, qui consiste pour le volontaire à suivre le véhicule qui le précède. Enfin, le troisième objectif était de poursuivre la tentative de corrélation entre les concentrations en THC et THC-COOH et la 
diminution des performances de conduite, qui avait été amorcée dans les deux études précédentes.

Un nouveau groupe de seize volontaires, comportant autant d'hommes que de femmes, a participé à cette étude. Afin de satisfaire aux règles de sécurité les plus strictes, on a adopté pour cette étude un protocole traditionnel. Autrement dit, les doses ont été administrées par ordre croissant. Pour chaque dose, des cigarettes normales et des cigarettes placebo ont été distribuées en double aveugle. Les valeurs des doses de THC étaient identiques à celles de l'étude précédente, soit 100,200 et $300 \mu \mathrm{g} / \mathrm{kg}$. A chaque fois les cigarettes avaient le même aspect, et elles étaient consommées à l'aide d'un fume-cigarette, chaque volontaire ayant pu choisir en l'espace de 10 minutes la façon dont il souhaitait fumer. Si un volontaire avait réagi de façon anormale à une dose inférieure, on ne l'aurait pas autorisé à prendre une dose plus forte.

Les volontaires ont commencé à fumer, à raison de deux à chaque fois. Trente minutes après avoir commencé à fumer, les deux premiers volontaires ont subi une batterie de tests en laboratoire (suivi de trajectoire, stabilité des mains et oscillations du corps), ont subi une prise de sang, puis ont évalué leur sensation de bien-être et autres sensations subjectives. Ensuite ils ont été transportés vers l'autoroute principale sur laquelle devaient se dérouler les tests de conduite. Nous disposions de deux véhicules équipés d'instruments. Pendant 12 minutes environ, les volontaires ont effectué sur une portion d'autoroute longue de $16 \mathrm{~km}$ le test qui consiste à suivre un véhicule. Ensuite ils ont tous les deux commencé le test de suivi de trajectoire, chacun conduisant un des véhicules équipés. L'autoroute était la même que pour le test précédent. Les volontaires ont parcouru $64 \mathrm{~km}$ sans s'arrêter pendant environ 50 minutes. Puis ils ont effectué une seconde fois le test de suivi de véhicule. Les volontaires ont ensuite été ramenés au laboratoire où ils ont évalué leurs sensations subjectives, subi un prélèvement sanguin, et se sont pliés à nouveau à la batterie de tests. Tout au long des deux types de tests, le rythme cardiaque des volontaires a été enregistré.

Le test de suivi de trajectoire était le même que celui de l'étude précédente, excepté sa durée et la présence de circulation sur l'autoroute. Les volontaires avaient pour consigne de rouler à une vitesse constante de $95 \mathrm{~km} / \mathrm{h}$ et de maintenir en permanence leur position latérale entre les deux bandes de la file de circulation située à droite. Ils avaient le droit de quitter leur position latérale uniquement s'ils étaient obligés de dépasser un véhicule plus lent roulant sur leur file. Les données du test standard ont été exploitées afin d'obtenir les mêmes critères de performance que dans l'étude précédente, c'est-à-dire la déviation standard de la position latérale, les écarts moyen et standard de vitesse, et l'écart angulaire standard du volant. Quant au test de suivi de véhicule, il sert à évaluer la capacité du conducteur à percevoir les changements de vitesse du véhicule qui le précède, ainsi que sa capacité de réaction afin de pouvoir conserver en permanence une distance de sécurité entre les deux véhicules. Au début du test, les 
deux véhicules roulaient en tandem sur la file de droite à une vitesse de 100 $\mathrm{km} / \mathrm{h}$, la voiture suivie étant conduite par un moniteur de conduite et la voiture suiveuse par le volontaire. Ce dernier avait pour instruction de garder une distance de sécurité de $50 \mathrm{~m}$, même si le premier véhicule modifiait sa vitesse. Après avoir roulé ainsi pendant une minute environ, le conducteur du premier véhicule a relâché la pédale d'accélérateur, réduisant sa vitesse à $80 \mathrm{~km} / \mathrm{h}$. Puis il a aussitôt accéléré, jusqu'à atteindre $100 \mathrm{~km} / \mathrm{h}$. Le temps nécessaire à une manoeuvre composée d'une décélération et d'une accélération était approximativement de 50 secondes. Au cours d'un test, six à huit manoeuvres ont pu être exécutées, selon l'intensité du trafic. Les variables dépendantes étaient le temps moyen de réaction du volontaire aux changements d'allure du véhicule suivi, la distance de sécurité moyenne et le coefficient de variation de la distance de sécurité au cours des manoeuvres.

Tous les volontaires ont pu effectuer cette série de tests sans problème particulier. Au cours du test standard de suivi de trajectoire les performances ont diminué avec l'augmentation de la dose de THC, ce qui est venu confirmer les résultats de l'étude précédente sur autoroute fermée à la circulation. La dose de $100 \mu \mathrm{g} / \mathrm{kg}$ a provoqué une augmentation légère, quoique presque significative, de la déviation standard moyenne de la position latérale. La dose de $200 \mu \mathrm{g} / \mathrm{kg}$ a provoqué une augmentation significative, dont la pertinence pratique est discutable. Quant à la dose de $300 \mu \mathrm{g} / \mathrm{kg}$, elle a provoqué une augmentation importante qui pourrait être considérée comme pertinente pratiquement. Cependant cette augmentation n'est pas exceptionnelle si on la compare aux effets qui sont provoqués par de nombreux médicaments. Après avoir fumé, les volontaires ont roulé à une vitesse moyenne qui n'était que légèrement inférieure à celle atteinte avec un placebo, et très proche de la vitesse moyenne imposée.

Toujours dans le cas du test de suivi de véhicule, pour toutes les doses les volontaires ayant consommé le placebo ont gardé une distance de sécurité de 45 à $50 \mathrm{~m}$. Les volontaires ont augmenté leur distance de sécurité de $8 \mathrm{~m}, 6 \mathrm{~m}$ et $3 \mathrm{~m}$ respectivement pour les doses de TCH de 100, 200 et $300 \mu \mathrm{g} / \mathrm{kg}$. Le fait que la différence entre drogue et placebo soit importante au départ et diminue avec l'augmentation de la dose est surprenant. Notre explication est que l'attention des volontaires était plus grande lors de la prise de la première dose, et qu'elle s'est atténuée au fur et à mesure de l'augmentation de la dose. Après consommation de marijuana, le temps de réaction aux variations de vitesse du véhicule suivi était plus important qu'avec un placebo. Mais plus la dose de THC administrée était importante, moins le temps de réaction a augmenté, comme pour la distance de sécurité. Cependant, l'augmentation du temps de réaction est en partie due à l'augmentation de la distance de sécurité. Un ajustement statistique a fait apparaître de plus faibles augmentations, non significatives, du temps de réaction après l'administration de marijuana, la plus forte augmentation ayant été observée après administration de la dose la plus faible de THC $(0,32 \mathrm{~s})$. La variabilité de la 
distance de sécurité a donné des résultats similaires à ceux obtenus pour la distance de sécurité moyenne et le temps de réaction; la plus grande variabilité a été observée après consommation de la dose la plus faible.

Un objectif pratique important de cette étude était de savoir s'il serait possible de prévoir l'importance de la diminution des performances de conduite, que ce soit à partir de la mesure des concentrations plasmatiques de THC ou à partir des performances de conduite évaluées lors de contrôles. Ceux-ci pourraient être mis en place au bord de la route et testeraient les capacités de suivi de trajectoire ou la stabilité des mains et du positionnement des conducteurs. Ces résultats, comme de nombreux autres auparavant, montrent qu'aucune de ces mesures ne permet de prévoir avec précision quelles seront les performances réelles du conducteur sous l'effet du THC.

Le Chapitre 8 est consacré à la troisième étude en conduite réelle prévue dans le programme de recherche. Cette étude est constituée de tests en zone urbaine à grande circulation. Seule la dose de $100 \mu \mathrm{g} / \mathrm{kg}$ a été administrée, pour des raisons de logique et de sécurité. Cette dose a été administrée, de même qu'un placebo, à un groupe de fumeurs réguliers de marijuana. A titre comparatif on a administré une petite dose de marijuana, avec des placebo également, à un autre groupe composé de consommateurs d'alcool réguliers aimant consommer cette drogue occasionnellement. Ils ont ensuite tous été soumis au même test de conduite en ville. Chaque groupe était composé de seize volontaires chacun, comportant autant d'hommes que de femmes. Les volontaires du second groupe consommaient régulièrement de l'alcool, mais seulement occasionnellement de la marijuana. Les deux groupes se sont vus administrer de la vraie drogue et des placebo. La drogue a été administrée par dose de THC de $100 \mu \mathrm{g} / \mathrm{kg}$. Le test de conduite a commencé 30 minutes après l'administration de la drogue. Quant à la dose d'alcool, elle a été calculée pour que les volontaires aient un taux d'alcoolémie de $0,5 \%$ au début du test, c'est-à-dire 45 minutes après avoir commencé à boire. La vraie drogue et les placebo ont été administrés en double aveugle et de façon alternée pour chacun des deux groupes.

Les tests de conduite ont tous eu lieu de jour, sur un trajet long de $17,5 \mathrm{~km}$, à l'intérieur de la ville de Maastricht. Les volontaires ont tous conduit un même jour de la semaine, à la même heure, avec des circulations de densité forte, moyenne et faible. Pour cette étude deux méthodes d'analyse ont été employées. La première, dite 'molaire', consiste pour le moniteur de conduite faisant office de contrôleur de sécurité lors des tests à évaluer rétrospectivement les performances du conducteur à l'aide d'une échelle standard. La seconde méthode, dite 'moléculaire', nécessite la présence d'un observateur formé à cet effet. En appliquant des critères simples et précis, celui-ci note les réactions du conducteur à certains points prédéterminés du parcours. Immédiatement avant et après les tests de conduite, les volontaires ont subi des tests de stabilité des mains et de 
perception temporelle, ainsi qu'un prélèvement sanguin, et ils ont dû répondre aux mêmes questionnaires subjectifs proposés dans les études précédentes.

L'étude a montré que par la méthode molaire une dose d'alcool modéréé (un taux d'alcoolémie de $0,3 \%$ ) réduisait de façon significative, comparée au placebo, les performances de conduite en zone urbaine. L'alcool affecte plus particulièrement le maniement de la voiture et la réalisation des manoeuvres. En revanche la marijuana, administrée en doses de $100 \mu \mathrm{g} / \mathrm{kg}$, n'a pas modifié de façon significative les performances moyennes mesurées suivant la méthode molaire. Cependant, suivant la méthode moléculaire les performances de conduite n'ont pas été affectées de façon significative, que ce soit par l'alcool ou par la marijuana, ce qui donne à penser que cette méthode est très peu sensible aux modifications provoquées par la drogue.

L'évaluation de la qualité de conduite par les volontaires a été différente de celle émise par l'observateur. Selon le moniteur, l'alcool a réduit les performances de conduite; mais les volontaires, eux, n'ent ont pas eu conscience. D'autres volontaires ont eu l'impression que la marijuana avait affecté leurs performances de conduite, alors que ce n'était pas le cas. Dans les deux groupes les volontaires ayant absorbé le placebo ont déclaré avoir éprouvé des difficultés à effectuer le test de conduite. Cependant, parmi les volontaires qui on inhalé la vraie drogue seuls les volontaires consommateurs de marijuana ont déclaré avoir fourni des efforts bien plus importants. Ceci prouve que non seulement les consommateurs de marijuana étaient conscients de leur intoxication, mais qu'ils essayaient aussi de compenser cet état. Pour nous, ces conclusions sont importantes. Elles viennent confirmer à la fois la réputation des conducteurs ayant bu de l'alcool d'être beaucoup trop sûrs d'eux, et également l'hypothèse des chercheurs selon laquelle les conducteurs ayant fumé une faible dose de marijuana sont plus prudents et remettent plus facilement leurs capacités en question.

Les résultats des tests de performance réalisés en laboratoire ont eux aussi été différents pour l'alcool et pour la marijuana. Par comparaison avec le placebo, la stabilité des mains s'est détériorée avec le THC et s'est améliorée avec l'alcool. La différence entre les effets des deux types de drogue a été tout à fait significative, tant avant le test de conduite qu'après. La diminution des performances observée après absorption de THC a été à peu près la même que lors de l'étude précédente pour la même dose, montrant ainsi que les membres des nouveaux groupes présentaient une sensiblilité équivalente à celle du groupe précédent. La production d'intervalles de temps n'a pas été affectée par l'alcool, en revanche le THC a réduit de façon importante la production d'intervalles, par comparaison avec le placebo.

On n'a pas trouvé de relation entre les concentrations de marijuana dans le plasma et les performances de conduite absolues, ni entre les concentrations plasmatiques et les variations observées selon que la vraie drogue ou le placebo a été administré. En ce qui concerne le THC, ces résultats viennent confirmer les 
conclusions des études antérieures. Pour ce qui est de l'alcool les résultats sont assez surprenants, mais ils s'expliquent peut-être par la faible étendue des concentrations plasmatiques en éthanol en fonction des différents volontaires.

Au Chapitre 9 nous concluons notre recherche en commentant de façon plus générale les résultats de notre programme de recherche. Nous terminons enfin par une liste de conclusions et recommandations. Ce chapitre commence par traiter la question de la dose de THC que les consommateurs de THC choisissent pour parvenir au bien-être. Nous soulevons plusieurs autre interrogations que nous argumentons ensuite, par exemple: comment les utilisateurs de marijuana ajustent-ils leur consommation de THC; quel est le rôle joué par la familiarisation avec cette drogue; quelle dose le fumeur aurait choisi si on lui avait donné de la marijuana bien plus concentrée. Nous prolongeons ensuite la discussion en analysant les différences qui existent entre les divers tests de conduite pour ce qui est de la nature du traitement de l'information requis (automatique ou dirigé), et en étudiant la pertinence de chaque type de traitement pour ce qui est de la sécurité routière.

Nous attirons ensuite à nouveau l'attention sur les effets du THC sur les performances de conduite. Les résultats des études que nous avons réalisées viennent corroborer les conclusions des tests sur simulateurs de conduite et circuit fermé réalisés auparavant. En effet, l'inhalation d'une dose de THC allant jusqu'à $300 \mu \mathrm{g} / \mathrm{kg}$ a sur les performances des effets négatifs importants, bien qu'ils ne soient pas dramatiques, liés à l'importance de la dose. Dans le cas du test de suivi de trajectoire, la déviation standard de la position latérale était la mesure la plus sensible permettant d'identifier les effets néfastes du THC. Ceci s'explique par le fait que le suivi de trajectoire est contrôlé principalement par un système automatique de traitement de l'information, qui ne dépend d'aucun moyen de contrôle conscient. Le processus est relativement peu sensible aux modifications de l'environnement, mais extrêmement sensible aux facteurs internes qui pourraient retarder le passage du flux d'information à travers le système. Le THC, ainsi que de nombreuses autres drogues, est un de ces facteurs. Lorsque ceux-ci interviennent dans le processus de réduction de la distance standard de la position latérale, l'individu ne peut pas faire grand chose pour y remédier. Dans le cas du suivi de véhicule et, dans une plus large mesure, de la conduite en ville, les performances de conduite dépendent plutôt d'un traitement de l'information dirigé, favorisant des mécanismes de compensation qui permettent de réduire ou même de supprimer totalement les diminutions de performances. Une autre conclusion importante découlant des études que nous avons réalisées est la suivante: la concentration plasmatique de THC et de THC-COOH mesurée dans un échantillon unique ne permet absolument pas de prévoir quoi que ce soit quant à la diminution des performances du conducteur.

Les effets de la marijuana sur les performances de conduite ont été comparés à ceux de nombreuses autres drogues. Il en est ressorti que les effets d'une dose 
de THC n'excédant pas $300 \mu \mathrm{g} / \mathrm{kg}$ ne dépassaient jamais les effets d'un taux d'alcoolémie de $0,8 \%$, et que ces effets n'étaient pas inhabituels du tout si l'on considère les effets de bon nombre de médicaments. Cependant, les effets du THC diffèrent qualitativement de beaucoup d'autres drogues, notamment l'alcool. Les présentes études ainsi que des études antérieures suggèrent que l'alcool favorise une comportement dangereux du conducteur alors que le THC entraîne une plus grande précaution, du moins au stade expérimental. Une autre caractéristique qualitative semble différencier le THC de bien d'autres types de drogues: sous son emprise de la drogue le conducteur aurait une plus grande capacité à compenser les effets néfastes du THC.

Il se trouve que la diminution des performances par le THC est plus importante en laboratoire que lors des tests en conduite réelle. Nous examinons donc plusieurs des raisons qui pourraient expliquer cette apparente incohérence. Premièrement, les moyens de commande des expérimentations en laboratoire font l'objet d'une grande simplification, si bien que le test peut sembler artificiel et affecter la motivation du volontaire. Deuxièmement, les tests en laboratoire n'offrent qu'un choix limité de manières de réagir pour le volontaire, ce qui limite les possibilités de compensation. Enfin, le volontaire possède un grand nombre de possibilités de compensation et c'est seulement en conditions réelles, ou avec des conditions très proches de la réalité, que l'on peut démontrer comment varient ces possibilités en fonction des effets de la drogue envisagée.

Cependant on peut facilement concevoir que dans certaines situtations l'action de la marijuana puisse avoir des conséquences très dangereuses; par exemple des situations d'extrême urgence qui exigeraient du conducteur une capacité très importante de traitement d'informations, des trajets très longs et monotones, ou l'association au THC d'autres drogues, notamment d'alcool. Ces éventualités étant tout à fait envisageables, il conviendra de ne pas considérer ce mémoire comme une fin en soi. Cette recherche devrait plutôt servir quelques temps de point de départ pour d'autres recherches, permettant ainsi d'enrichir la connaissance que l'on a des effets du THC sur les performances de conduite. 


\section{ZUSAMMENFASSUNG}

In dieser Dissertation werden die Wirkungen des Marihuanarauchens auf die tatsächliche Fahrtüchtigkeit beschrieben. Sie enthält Überblicke über die allgemeinen Eigenschaften des Hauptbestandteils von Marihuana, $\Delta^{9}$-Tetrahydrocannabinol (THC), und vorausgehende Untersuchungen über die Auswirkungen auf die Fahrtüchtigkeit und Verkehrssicherheit. Außerdem wird ein Forschungprogramm beschrieben, bestehend aus einer Labor- und drei Fahrstudien, in denen das Verhältnis Dosis-Wirkung in tatsächlichen, der Realität stufenweise angeglichenen Fahrsituationen gemessen wurde. Hauptziel war es, zu bestimmen, wieviel THC bei Steigerung der Dosis die Fahrtüchtigkeit beeinflußt und welche Auswirkungen dies auf die Verkehrssicherheit haben könnte. Außerdem sollte ermittelt werden, ob es möglich ist, Veränderungen der Fahrtüchtigkeit mit THCPlasmakonzentrationen oder Plasmakonzentrationen seines hauptsächlichen (inaktiven) Metaboliten, der THC-Carbonsäure (THC-COOH), in Beziehung zu setzen. Eine Reihe von Fahrtests wurde ausgearbeitet, zu denen unter anderem das Einhalten einer konstanten Geschwindigkeit und einer gleichmäßigen Position zum Mittelstreifen bei ununterbrochenem Autobahnverkehr, dem Folgen eines voranfahrenden Fahrzeugs bei unterschiedlichen Geschwindigkeiten auf der Autobahn, sowie ein Fahren im Stadtverkehr gehörte. Mit diesen Fahrtests sollte bestimmt werden, ob bei allen Versuchspersonen ähnliche Veränderungen der Fahrtüchtigkeit unter Einfluß von THC auftreten, wodurch auf eine allgemeine Wirkung von Drogen auf die Fahrtüchtigkeit geschlossen werden könnte.

Die Kapitel 1 und 2 enthalten die Begründung für die Dissertation beziehungsweise einen allgemeinen Überblick über Cannabis. Anschließend werden die Geschichte des Cannabiskonsums, die gegenwärtig bevorzugten Anwendungsformen, Chemie und Pharmakologie, akute und chronische Wirkungen sowie therapeutische Anwendungen erörtert.

Kapitel 3 bezieht sich auf fachspezifische Literatur über die Auswirkungen von Cannabis auf das Führen von Fahrzeugen. Epidemische Untersuchungen zeigen, daß Menschen nach dem Genuß von Cannabis Auto fahren und daß an Unfällen beteiligte Fahrer vorher oft die Droge genommen haben. Die Resultate 
sind jedoch besonders wegen der großen Zahl der Fälle nicht überzeugend, bei denen auch Alkohol im Spiel war, und auch, weil keine geeigneten Kontrollgruppen vorhanden waren. Das Maß, in dem Cannabis als Ursache von Verkehrsunfällen beiträgt, bleibt unklar. Ergebnisse aus Experimenten mit Fahrsimulatoren sowie aus Versuchen auf abgesperrten Strecken verdeutlichen, daß THC in einmalig inhalierten Dosierungen bis zu ungefähr $250 \mu \mathrm{g} / \mathrm{kg}$ nur geringe Auswirkung auf die Fahrtüchtigkeit hat, sicherlich aber weniger als eine Blutalkoholkonzentration (BAK) von 0,8-1,0\%. Im Gegensatz dazu haben Laborversuche wiederholt ergeben, daß eine Leistungsverschlechterung eintritt, nach dem so geringe Dosierungen wie $40 \mu \mathrm{g} / \mathrm{kg}$ inhaliert wurden. Die Beeinträchtigung wird nach Dosierungen von 100-200 $\mu \mathrm{g} / \mathrm{kg}$ größer und hält länger an. Die Wirkungen von THC beeinflußten besonders die Leistungsfähigkeit in Experimenten zum Halten der Spur, zur geteilten Aufmerksamkeit sowie zur Wachsamkeit.

In Kapitel 4 wird das Programm einer Labor- und dreier Fahrstudien beschrieben sowie bestimmte Voraussetzungen, die bei allen Experimenten gleich waren. Dabei handelte es sich um die Einhaltung von moralischen und gesetzlichen Normen, die Anwerbung von Versuchspersonen, Marihuana-Zigaretten und Rauchverfahren, Überprüfung des Konsums von anderen illegalen Drogen und Alkohol während der Tests, sowie Verfahren für Blutproben und quantitative Analysen. Die an allen Studien beteiligten Versuchspersonen waren Cannabis-Gelegenheitsraucher, d.h. sie rauchen Marihuana oder Haschisch mehr als einmal im Monat, aber nicht täglich. Alle Personen waren gesund, zwischen 21 und 40 Jahre alt, hatten normales Gewicht und verfügten über binokulare Sehkraft; alle besaßen eine gültige Fahrerlaubnis. Die Versuchspersonen wurden auf jeder Versuchsfahrt von einem geprüften Fahrlehrer begleitet. Die Versuchsfahrzeuge waren für eventuell auftretende Notfälle mit zusätzlichen Kontrollsystemen ausgerüstet. Marihuana- sowie Placebo-Marihuana-Zigaretten wurden vom U.S. National Institute on Drug Abuse zur Verfügung gestellt.

Kapitel 5 enthält die Ergebnisse der Laborstudie. Unter strenger medizinischer Überwachung wurde in einem Krankenhaus die Studie zur Ermittlung der THCDosierungen durchgeführt, die Gelegenheitsraucher von Marihuana wahrscheinlich vor dem Fahren eines Wagens konsumieren. Es nahmen vierundzwanzig Versuchspersonen teil, zwölf Männer und zwölf Frauen. Sie durften bis zu drei Marihuana-Zigaretten rauchen, um die von ihnen gewünschte psychologische Wirkung zu erzielen. Die Zigaretten wurden auf die von den Versuchspersonen bevorzugte Art durch eine Spitze aus Plastik geraucht. Einzige Bedingung war, daß ununterbrochen für eine Zeitraum von nicht mehr als 15 Minuten geraucht wurde. Nachdem die Personen freiwillig das Rauchen beendeten, wurden die Zigaretten sorgfältig ausgemacht und für eine anschließende gravimetrische Bestimmung des konsumierten THCs aufbewahrt. Sechs Personen rauchten eine Zigarette, dreizehn rauchten zwei und vier Personen rauchten drei Zigaretten. Die durchschnittliche Menge des konsumierten THC betrug 20,8 mg; unter 
Berücksichtigung des Körpergewichts lag sie bei $308 \mu \mathrm{g} / \mathrm{kg}$. In bezug auf die, dem Körpergewicht angeglichene Dosis wurden zwischen Frauen und Männern sowie zwischen Personen, die häufig rauchen, und Gelegenheitskonsumenten keine besonderen Unterschiede festgestellt. Es wurde beschlossen, die maximale Dosis für die anschließenden Experimente zur Fahrtüchtigkeit auf $300 \mu \mathrm{g} / \mathrm{kg}$ festzusetzen, was erheblich über den Dosierungen liegt, die Versuchspersonen normalerweise bei experimentellen Studien erhalten (normal sind 100-200 $\mu \mathrm{g} / \mathrm{kg}$ THC).

Die Studie bot die Möglichkeit, wertvolle Informationen über die Pharmakokinetik von THC und seine pharmakodynamische Auswirkung nach dem Rauchen von Marihuana zu erhalten. Wiederholt wurden Blutproben genommen, um die Plasmakonzentration von THC und THC-COOH zu messen. Die Versuchspersonen durchliefen wiederholt bestimmte einfache Laborversuche, schätzten ihren Rauschzustand ein und erklärten ihre Bereitschaft, in einigen besonders spezifizierten Streßsituationen zu fahren, wobei die Herzfrequenz gemessen wurde. Zweites Ziel der Laborstudie war es, die genaueren Beziehungen $\mathrm{zwischen}[\mathrm{THC}] \mathrm{bzw}$. [THC- $\mathrm{COOH}$ ] und den Veränderungen in den anderen physiologischen, leistungsbezogenen oder subjektiven Variablen zu untersuchen. Andere Ergebnisse aus der Laborstudie zeigten, daß der wahrgenommene Rauschzustand und die Herzfrequenz sehr sensible Parameter für die Messung der Marihuana-Intoxikation sind, was frühere Resultate bestätigt. Eine Beeinträchtigung der Leistungsfähigkeit in den Laborversuchen wurde zum Zeitpunkt des emotionalen Höhepunkts der Versuchspersonen festgestellt, im allgemeinen aber löst sich eine objektive Beeinträchtigung schneller auf als die von ihr hervorgerufenen Emotionen selbst.

Die erste Fahrstudie, die in Kapitel 6 beschrieben wird, wurde auf einer für den übrigen Verkehr gesperrten Autobahn durchgeführt. Ein Ziel der Studic war es, festzustellen, ob es ungefährlich wäre, die Studie auf einer normalen Autobahn bei normalem Verkehr zu wiederholen. Das zweite Ziel bestand darin, das Verhältnis von Dosis und Wirkung zwischen der inhalierten THC-Dosis und der Fahrtüchtigkeit zu bestimmen. Die Versuchspersonen waren die gleichen zwölf Frauen und zwölf Männer, die auch schon an der Laborstudie teilgenommen hatten. Ihnen wurden zu verschiedenen Gelegenheiten THC-Dosierungen von 0, 100,200 und $300 \mu \mathrm{g} / \mathrm{kg}$ verabreicht. Die Behandlungen erfolgten doppelblind und in ausgleichender Reihenfolge. Die Versuchspersonen legten jedesmal einen Test zum Halten der Spur ab, der 40 Minuten nach der Beendigung des Rauchens begann und eine Stunde später wiederholt wurde. Der Versuch, der von O'Hanlon u.a. $(1982,1986)$ entwickelt und genormt wurde, umfaßt des Einhalten einer konstanten Geschwindigkeit von $90 \mathrm{~km} / \mathrm{h}$ bei einem gleichmäßigen Abstand zwischen den Markierungen der Fahrspur. Die Versuchspersonen fuhren $22 \mathrm{~km}$ auf einer Autobahn und wurden von einem geprüften Fahrlehrer begleitet. Dieser war für die ständige Sicherheit verantwortlich und konnte, wenn nötig, durch zusätzliche Kontrollsysteme im Fahrzeug eingreifen. Die wesentliche Haupt- 
variable war die Normabweichung von der parallelen Position zu den Fahrspurbegrenzungen (im folgenden: SDLP, standard deviation of lateral position), die sich als sowohl höchst abhängig von als auch sehr empfindlich für den Einfluß von sedativen Arzneimitteln und Alkohol erwiesen hat. Andere wesentliche Variablen waren die durchschnittliche Geschwindigkeit sowie Normabweichungen von der mittleren Geschwindigkeit und dem Lenkrad-Winkel. Vor jedem Fahrversuch wurden Blutproben entnommen; nach Beendigung des Versuchs wurde die Leistungsfähigkeit beim genauen Spurhalten und in Versuchen zum Ruhighalten der Hand gemessen, sowie Herzfrequenz und Blutdruck. Wiederholt mußten Fragebögen zur Einschätzung des Rauschgefühls und anderen subjektiven Emotionen ausgefüllt werden.

Alle Versuchspersonen wollten und konnten die Versuche ohne große Schwierigkeiten ausführen. Die Studie zeigte, daß Marihuana die Fahrtüchtigkeit beeinträchtigt, was durch ein Ansteigen des Parameters SDLP gemessen wurde. Im Vergleich zum Placebo beeinflußten alle drei Dosierungen des THC die SDLP entscheidend. Die Beeinträchtigung der Fahrtüchtigkeit nach dem Marihuanakonsum hielt fast unverändert noch zwei Stunden an, während die Drogenkonzentration im Plasma, der wahrgenommene Rauschzustand und der Anstieg der Herzfrequenz abgenommen hatten. Die Wirkung von Marihuana auf die SDLP wurde mit der von Alkohol verglichen, die von Louwerens u.a. $(1985,1987)$ in einer sehr ähnlichen Studie untersucht wurde. Dabei ergab sich, daß die Wirkungen von THC auf die SDLP gleich denen der Blutalkoholkonzentration (BAK) in einem Bereich von 0,3-0,7\% waren. Andere Parameter zur Feststellung der Fahrtüchtigkeit wurden von THC kaum beeinflußt. Innerhalb der Gruppe waren die Wechselbeziehungen zwischen den Plasmakonzentrationen der Droge und der Fahrtüchtigkeit nach jeder Dosis im wesentlichen gleich null. Also kann von den vorhandenen Plasmakonzentrationen von THC bzw. THC-COOH nicht auf das Maß der Beeinträchtigung der Fahrtüchtigkeit geschlossen werden. Die Beeinträchtigung der Fahrtüchtigkeit wurde auch nicht zu der Leistungsfähigkeit in den Laborversuchen in Beziehung gesetzt. Die festgestellte Beeinträchtigung der Fahrtüchtigkeit und das Verhalten der Versuchspersonen zeigte, daß ein normaler Schutz zur Gewährleistung der Sicherheit bei weiteren Versuchen ausreichen würde. Daraufhin wurde beschlossen, diese Studie auf einer normalen Autobahn bei normalem Verkehr zu wiederholen.

Die in Kapitel 7 beschriebene zweite Studie wurde durchgeführt, um der Realität näher zu kommen, als dies bei der ersten möglich war. Die Versuche wurden nun auf einer Autobahn mit normalem Verkehr durchgeführt. Hauptziel dieser Studie war es, die Beziehung zwischen der inhalierten THC-Dosis und den Abweichungen von der parallelen Position in einem genormten Versuch zum Spurhalten zu bestätigen. Als zweites Ziel sollte in einem anderen Fahrversuch die Leistungsfähigkeit beim Folgen eines anderen Wagens gemessen werden. Und drittens sollten die Bemühungen fortgesetzt werden, die Plasmakonzentrationen 
von THC und THC-COOH mit der Beeinträchtigung der Fahrtüchtigkeit, wie sie in beiden Versuchen gemessen wurde, miteinander in Beziehung zu setzen.

Eine neue Gruppe von sechzehn Versuchspersonen (acht Männer und acht Frauen) nahm an dieser Studie teil. Bei der Erstellung der vorliegenden Studie wurde ein vorsichtiger Ansatz gewählt, um die strengsten Erfordernisse an die Sicherheit zu gewährleisten. Das heißt, die Studie wurde auf der Basis einer ansteigenden Serie von Dosierungen durchgeführt, wobei im Doppelblindversuch sowohl die wirksame Droge als auch die Placebos in jeder Größenordnung der drei THC-Dosierungen verabreicht wurden. Die THC-Dosierungen waren die gleichen wie die in der ersten Studie, nämlich 100, 200 und $300 \mu \mathrm{g} / \mathrm{kg}$. Die Zigaretten konnten von den Versuchspersonen in keinem Versuchsstadium voneinander unterschieden werden und wurden in einer von der Versuchsperson bevorzugten Art durch eine Spitze aus Plastik innerhalb einer Zeit von 10 Minuten geraucht. Wenn eine Versuchsperson auf eine niedrige Dosis unakzeptabel reagiert hätte, wäre es ihr nicht erlaubt worden, eine höhere Dosis einzunehmen.

Immer zwei Versuchspersonen beendeten das Rauchen zu gleicher Zeit. Dreißig Minuten nach Beginn des Rauchens durchliefen die Versuchspersonen eine Reihe von Laborversuchen ('tracking', Versuche zum Ruhighalten der Hand und zur Körperkontrolle), gaben eine Blutprobe ab und schätzten ihren Rauschzustand und andere subjektive Emotionen ein. Sie wurden dann zu einem Autobahnabschnitt gebracht, auf dem die Fahrversuche stattfanden. Zwei mit Instrumenten bestückte Fahrzeuge standen zur Verfügung. Die Versuchspersonen legten den Test zum Folgen eines voranfahrenden Fahrzeugs auf einem $16 \mathrm{~km}$ langen Teilstück der Autobahn in ungefähr zwölf Minuten ab. Nach der Beendigung dieses Versuchs begannen beide Versuchspersonen mit dem Test zum Spurhalten in gesonderten, mit Instrumenten versehenen Wagen. Das zu befahrende Teilstück der Autobahn war das gleiche wie bei dem ersten Test. Die Versuchspersonen fuhren ohne Anhalten $64 \mathrm{~km}$ in ungefähr 50 Minuten. Nach Beendigung dieses Versuchs nahmen beide Teilnehmer wieder an dem Versuch zum Folgen eines anderen Wagens teil. Anschließend wurden sie wieder zum Labor gebracht, wo sie ihre persönlichen Emotionen einschätzten, eine Blutprobe abgaben und erneut die obige Reihe von Versuchen durchliefen. Bei beiden Fahrversuchen wurde die Herzfrequenz der Versuchspersonen ununterbrochen aufgezeichnet.

Der Versuch zum Spurhalten war mit Ausnahme seiner Dauer und der Anwesenheit von anderem Verkehr der gleiche wie in der vorhergehenden Studie. Die Versuchspersonen wurden instruiert, eine konstante Geschwindigkeit von $95 \mathrm{~km} / \mathrm{h}$ sowie auf der rechten Fahrbahn eine gleichmäßige parallele Position zum Mittelstreifen zu halten. Eine Abweichung wurde ihnen nur dann gestattet, wenn es nötig sein würde, ein sich auf der gleichen Spur befindendes langsameres Fahrzeug zu überholen. Die Daten aus dem Standardversuch wurden 
analysiert, um die gleichen Leistungsparameter wie in der vorherigen Studie zu erhalten, d.h. SDLP, durchschnittliche Geschwindigkeit und Normabweichungen von der mittleren Geschwindigkeit sowie Abweichungen vom Lenkrad-Winkel. Der Test zum Folgen eines Fahrzeugs mißt die Fähigkeit des Fahrers, Veränderungen in der Geschwindigkeit eines voranfahrenden Fahrzeugs wahrzunehmen und entsprechend so zu reagieren, daß ein gleichmäßiges Fahren gewährleistet ist. Er begann, als das vorausfahrende und das nachfolgende Fahrzeug, das jeweils von einem der Fahrlehrer und der Testperson gefahren wurde, hintereinander auf der Spur für den langsameren Verkehr mit einer Geschwindigkeit von $100 \mathrm{~km} / \mathrm{h}$ fuhren. Die Versuchsperson sollte einen Abstand von 50 Metern halten, wie auch immer sich die Geschwindigkeit des vorausfahrenden Fahrzeuges ändern würde. Nach dem auf diese Weise ungefähr eine Minute lang gefahren wurde, nahm der Fahrer des vorausfahrenden Fahrzeugs den Fuß vom Gaspedal, so daß die Geschwindigkeit des Wagens auf $80 \mathrm{~km} / \mathrm{h}$ sank. Direkt anschließend beschleunigte er auf $100 \mathrm{~km} / \mathrm{h}$. Die Geschwindigkeitsreduzierung sowie die folgende Beschleunigung nahmen annähernd 50 Sekunden in Anspruch; sechs bis acht solche Manöver wurden je nach Verkehrslage in einem Versuch durchgeführt. Die durchschnittliche Reaktionszeit der Versuchspersonen auf die Bewegungen des vorausfahrenden Fahrzeug, der durchschnittliche Abstand und der Variationskoeffizient des Abstands während der Manöver wurden als die wesentlichen Variablen gemessen.

Alle Versuchspersonen konnten die Testreihe beenden, ohne irgendeine unglückliche Reaktion beim Fahren zu zeigen. Die Leistungsfähigkeit beim Spurhalten im Standardversuch wurde von der jeweiligen THC-Dosis beeinträchtigt und bestätigte die Ergebnisse der vorherigen, auf einem abgesperrten Teilstück der Autobahn abgehaltenen Untersuchung. Die $100 \mu \mathrm{g} / \mathrm{kg}$ Dosis rief eine leichte Erhöhung der durchschnittlichen SDLP hervor, wenngleich sie auch kaum von Bedeutung war. Die $200 \mu \mathrm{g} / \mathrm{kg}$ Dosis bewirkte eine deutliche Erhöhung von jedoch zweifelhafter praktischer Relevanz. Die $300 \mu \mathrm{g} / \mathrm{kg}$ Dosis produzierte eine bedeutende Erhöhung der SDLP, die als für die Praxis relevant betrachtet werden muß, jedoch nur durchschnittlich im Vergleich $\mathrm{zu}$ ähnlichen festgestellten Wirkungen vieler anderer Arzneimittel ist. Die im nachfolgenden Wagen fahrenden Versuchspersonen, die Marihuana geraucht hatten, fuhren mit einer durchschnittlichen Geschwindigkeit, die nur etwas niedriger lag als nach Verabreichung der Placebos und sehr nahe an vorgeschriebenen Grenze lag.

Im Versuch zum Folgen eines Wagens unter Placebo-Bedingungen hielten die Versuchspersonen einen Abstand von 45-50 Metern ein. Unter entsprechenden THC-Bedingungen von 100,200 beziehungsweise $300 \mu \mathrm{g} / \mathrm{kg}$ verlängerten sie den durchschnittlichen Abstand um 8,6 und 2 Meter. Der anfängliche große Unterschied zwischen der Leistungsfähigkeit bei der Droge und dem Placebo und der anschließender Rückgang ist ein erstaunliches Ergebnis. Unsere Erklärung für diese Beobachtung ist, daß die Vorsicht der Versuchspersonen am größten war, 
als sie den Test unter dem Einfluß von THC zum ersten Mal ablegten, und daß die Vorsicht nachher immer weiter abnahm. Die Reaktionszeiten in bezug auf die Geschwindigkeitsveränderungen des vorausfahrenden Fahrzeugs erhöhten sich im Vergleich zum Placebo nach einer Einnahme von THC. Die verabreichte THCDosis war umgekehrt proportional zur Veränderung in der Reaktionszeit, wie sie es beim Abstandhalten war. Die verlängerten Reaktionszeiten waren jedoch teilweise auf einen größeren Abstand zurückzuführen. Die statistische Angleichung dieser verwirrenden Tatsache führte zu kleinerem und unbedeutendem Anstiegen der Reaktionszeit nach Marihuanagenuß; die größte Beeinträchtigung im ersten Test $(0,32 \mathrm{~s})$ wurde bei der geringsten THC-Dosis festgestellt. Die Veränderungen beim Abstandhalten folgten einem ähnlichen Muster wie bei dem durchschnittlichen Abstandhalten und der Reaktionszeit; die größte Beeinträchtigung wurde nach der geringsten Dosis festgestellt.

Ein wichtiges praktisches Ziel dieser Studie war es, zu bestimmen, ob gewisse Grade in der Beeinträchtigung der Fahrtüchtigkeit genau vorausbestimmt werden können, und zwar entweder durch ein Messen der THC-Konzentration im Plasma oder durch ein Messen der Leistungsfähigkeit in entsprechenden 'Nüchternheitstest' auf der Straße zur Feststellung der Fähigkeit des 'trackings', oder der Hand- und Körperbeherrschung. Wie andere Untersuchungen bereits gezeigt haben, verdeutlichen die Ergebnisse, daß sich durch keine dieser Untersuchungen die Veränderungen der tatsächlichen Fahrleistung unter dem Einfluß von THC genau voraussagen lassen.

Das Programm ging dann zum dritte Fahrversuch über, der in Kapitel 8 beschrieben wird, und bei dem die betreffenden Tests in dichtem Stadtverkehr durchgeführt wurden. Aus logischen und sicherheitstechnischen Gründen wurde die THC-Dosis auf $100 \mu \mathrm{g} / \mathrm{kg}$ beschränkt. Diese Dosis wurde zusammen mit Placebos einer Gruppe von regelmäßigen Cannabiskonsumenten verabreicht. Aus Gründen der Vergleichbarkeit wurde einer anderen Gruppe von regelmäßig Alkohol konsumierenden Personen neben Placebos eine bescheidene Dosis der von ihnen bevorzugten Droge gegeben, bevor mit ihnen der gleiche Test zur Fahrt im Stadtverkehr durchgeführt wurde. Zwei Gruppen von jeweils sechzehn neuen Versuchspersonen, die zu gleichen Teilen aus Männern und Frauen bestanden, nahmen daran teil. Die Personen der Alkohol-Gruppe nahmen regelmäßig Alkohol, aber kein Marihuana. Beide Gruppen erhielten bei verschiedenen Gelegenheiten die aktive Droge bzw. Placebos. Aktives Marihuana wurde verabreicht, um eine Dosis von $100 \mu \mathrm{g} / \mathrm{kg}$ zu erreichen. Der Fahrversuch begann 30 Minuten nach dem Rauchen. Die Alkohol-Dosis wurde so gewählt, daß sie eine BAK Annäherung von 0,5\% hervorrief, wenn der Fahrversuch 45 Minuten nach Beendigung des Trinkens stattfand. Die wirksame Droge und die Placebos wurden in einem Doppelblindversuch verabreicht und gleichgewichtig in jeder Gruppe verteilt. 
Die Fahrversuche wurden bei Tageslicht auf einer ununterbrochenen Strecke von $17,5 \mathrm{~km}$ innerhalb der Stadtgrenzen der niederländischen Stadt Maastricht durchgeführt. Die Versuchspersonen machten ihre Fahrten mit der aktiven Droge sowie mit den Placebos bei starkem, mittlerem und wenig Verkehr am gleichen Wochentag zur gleichen Tageszeit. Für die vorliegende Studie wurden zwei Beurteilungsmethoden entwickelt. Bei der ersten, der 'molaren' Methode, mußte der Fahrlehrer während der Tests als Sicherheitskontrolleur fungieren, um die Leistung des Fahrers anschließend mittels einer Standardtabelle einzuschätzen. Die zweite, eher 'molekulare' Methode, beinhaltete den Einsatz eines besonders geschulten Beobachters, der einfache und strenge Kriterien anwandte, um festzuhalten, ob der Fahrer jeweils eine Reihe von erkennbaren Reaktionen an vorbestimmten Stellen der ausgewählten Strecke machte oder auch nicht. Direkt vor und nach den Fahrtests machten die Versuchspersonen Versuche zur Handbeherrschung und Zeitwahrnehmung, gaben eine Blutprobe ab und mußten die gleichen Fragebögen wie in den vorangegangenen Studien ausfüllen.

Die Studie zeigte, daß eine geringe Dosis Alkohol (BAK=0,3\%) eine bedeutende Beeinträchtigung der Fahrtüchtigkeit im Stadtverkehr hervorrief, wie auch bei der molaren Methode im Vergleich zum Placebo festgestellt wurde. Um genauer zu sein, der Alkohol verschlechterte die Fähigkeit, mit dem Wagen umzugehen und Fahrmanöver auszuführen. Marihuana hingegen, das in einer Dosis von $100 \mu \mathrm{g} / \mathrm{kg}$ verabreicht wurde, beeinflußte die durchschnittliche Fahrtüchtigkeit unerheblich, wie mit dieser Methode bewiesen wurde. Weder Alkohol noch Marihuana beeinflußten die in der molekularen Methode gemessenen Daten zur Fahrleistung maßgeblich, was andeutet, daß sie für von Drogen hervorgerufene Veränderungen relativ unempfindlich ist.

Die Beurteilung der Fahrleistung durch die Versuchspersonen unterschied sich von der des Beobachters. Dem Fahrlehrer zufolge beeinträchtigte Alkohol die Fahrleistung, was die Versuchspersonen aber nicht wahrnahmen; Marihuana hatte keine negativen Auswirkungen auf die Fahrtüchtigkeit, die Versuchspersonen selbst aber empfanden sie als beeinträchtigt. Beide Gruppen berichteten über das gleiche Maß an Anstrengung, den an das Placebo anschließenden Fahrversuch zu bewältigen. Jedoch nur Personen aus der Marihuana-Gruppe zeigten bedeutend höhere Anstrengungen nach Verabreichung der aktiven Droge. Es lag also ein Beweis dafür vor, daß sich die Versuchspersonen der Marihuana-Gruppe ihrer Intoxikation nicht bewußt waren, aber gleichzeitig versuchten, sie zu kompensieren. Dies scheinen wichtige Beobachtungen zu sein. Beide unterstützen sie die allgemeine Ansicht, daß Autofahrer nach Alkoholgenuß übertrieben selbstsicher werden, sowie den Verdacht der Forscher, daß Personen nach einer niedrigen Dosis THC durch Marihuanakonsum dagegen vorsichtiger und selbstkritischer werden.

Die Labortests zur Leistungsfähigkeit machen bei den Wirkungen der Droge Unterschiede. Die Handbeherrschung wurde im Vergleich zum Placebo nach 
einer THC-Dosis beeinträchtigt und nach Alkohol verbessert. Die Beeinträchtigung nach THC lag in dem gleichen Maß vor wie die, von der gleichen Dosis hervorgerufene Beeinträchtigung in der ersten Studie, was auf ein gleiches Maß an Sensibilität in der jetzigen und der vorherigen Gruppe hindeutet. Eine Produktion von Zeitintervallen wurde von Alkohol nicht beeinflußt, aber THC verkürzte im Vergleich zum Placebo die Intervallproduktion erheblich.

Die Drogenkonzentrationen im Plasma wurden weder in Beziehung zu den Angaben zur absoluten Fahrleistung gesetzt noch zu den Veränderungen, die beim Wechsel vom Placebo zur Droge auftraten. In bezug auf THC bestätigen diese Ergebnisse die Resultate aus vorangehenden Studien. Im Falle von Alkohol sind sie leicht überraschend, könnten aber auf begrenzte, unterschiedliche Ethanolkonzentrationen im Plasma von verschiedenen Versuchspersonen zurückgeführt werden.

Kapitel 9 beschließt den Bericht mit einer allgemeinen Erörterung der Ergebnisse des Programms und endet mit einer Reihe von Schlußfolgerungen und Empfehlungen. Es beginnt mit einer Darlegung über die THC-Dosis, die Marihuanaraucher zur Erzeugung des gewünschten Rauschzustands im allgemeinen bevorzugen. Verschiedene Fragen werden gestellt und erörtert, so z.B. wie regulieren Personen ihren THC-Konsum; welche Rolle spielt eine Gewöhnung an die Droge; wie wäre die bevorzugte Dosis gewesen, wenn Marihuana mit einer viel höheren Wirksamkeit geraucht worden wäre. Die Erörterung fährt dann mit einer Beschreibung der Unterschiede zwischen den Fahrversuchen fort, und zwar in bezug auf die Art der Informationserzeugung, die jeder Tests erfordert, automatisch oder kontrolliert, und die jeweilige Bedeutung für die Verkehrssicherheit.

Weiterhin richtet sich die Aufmerksamkeit auf die Auswirkungen von THC auf die Fahrtüchtigkeit. Die Ergebnisse der Studien bestätigen die Resultate aus den vorangehenden Tests in der Fahrsimulation und auf der abgesperrten Strecke, indem sie aufzeigen, daß THC in einmalig inhalierter Dosis von bis zu $300 \mu \mathrm{g} / \mathrm{kg}$ deutliche, aber nicht dramatische dosisabhängige beeinträchtigende Auswirkungen auf die Fahrtüchtigkeit hat. Die Abweichung vom Mittelstreifen (SDLP) beim Test zum Spurhalten war der empfindlichste Parameter für das Erkennen der negativen Wirkungen von THC, und zwar aus dem Grund, daß das Spurhalten in erster Linie von einem automatischen Informationserzeugungssystem kontrolliert wird, daß außerhalb der bewußten Kontrolle arbeitet. Der Prozeß ist relativ unzugänglich für äußere Veränderungen, aber reagiert sehr empfindlich auf innere Faktoren, die den Informationsfluß innerhalb des Systems verzögern. THC und viele andere Drogen gehören zu diesen Faktoren. Wenn sie den Prozeß stören, beeinflußt das die SDLP negativ. Es gibt dann wenig, was die betroffenen Personen durch Kompensation tun können, um die ursprüngliche Situation wiederherzustellen. Die Leistungsfähigkeit beim Folgen eines Wagens und, in größerem Ausmaße, die Leistungsfähigkeit im Stadtverkehr hängt mehr von einem kontrol- 
lierten Informationsprozeß ab und ist deshalb eher zugänglich für kompensierende Mechanismen, die die Beeinträchtigungen abmildern oder sie ganz verschwinden lassen. Eine weitere wichtige Schlußfolgerung der vorliegenden Studie ist, daß man aufgrund der Plasmakonzentration von THC und THC- $\mathrm{COOH}$, die aus einer einzigen Probe bestimmt wurde, unmöglich etwas über die Beeinträchtigung des Fahrers sagen kann.

Die Auswirkungen von Marihuana auf die Fahrtüchtigkeit wurden mit denen anderer Arnzneimitteln und Alkohol verglichen. Es wurde gefolgert, daß die Wirkungen von THC nach Dosierungen von mehr als $300 \mu \mathrm{g} / \mathrm{kg}$ nie die von Alkohol in einer BAK von $0,8 \%$ übersteigen, und im Vergleich $\mathrm{zu}$ vielen Arzneimitteln keinesfalls unüblich waren. Dennoch unterscheiden sich die Wirkungen von THC qualitativ von vielen anderen Drogen. Erkenntnisse aus der vorliegenden und aus früheren Studien legen den Schluß nahe, daß, zumindest in Experimenten, Alkohol eine riskantere Fahrweise, THC jedoch eine größere Vorsicht zur Folge hat. THC scheint sich auch auf eine andere Art qualitativ von anderen Drogen zu unterscheiden: Die Konsumenten scheinen eher dazu fähig zu sein, die ungünstigen Wirkungen beim Fahren unter Drogeneinfluß zu kompensieren.

Es scheint, daß THC die Fahrtüchtigkeit eher in den Laborversuchen als in den tatsächlichen Fahrexperimenten beeinflußt. Verschiedene, diese offensichtliche Diskrepanz eventuell erklärende Gründe wurden besprochen. Erstens werden die Laborversuche experimentell durch drastische Vereinfachungen kontrolliert, was die Motivation einer Versuchsperson, den Test zu bestehen, beeinflussen könnte, weil ihr der Test 'unrealistisch' erscheint. Zweitens läßt die Beschränkung der Reaktionsmöglichkeiten in den Laborversuchen zur Fahrtüchtigkeit weniger Möglichkeiten zur Kompensation. Und schließlich besitzen die Versuchspersonen, nachdem sie Fahren gelernt haben, solche Fähigkeiten in Hülle und Fülle, und es kann nur aufgezeigt werden, wie sie bei der wirklichen Aufgabe unter Drogeneinfluß variieren, bzw. nur eine sehr nahe Annäherung daran.

Man kann sich jedoch leicht Situationen vorstellen, in denen der Einfluß von gerauchtem Marihuana eine darüber hinausgehende gefährliche Wirkung haben könnte, d.h. Notsituationen, die große Anforderungen an die Fähigkeit des Fahrers stellen, Informationen zu verarbeiten; längeres monotones Fahren; und die Einnahme von THC in Verbindung mit anderen Drogen, besonders Alkohol. Weil diese Möglichkeiten der Wirklichkeit entsprechen, sollte diese Dissertation nicht als Schlußwort betrachtet werden. Sie sollte jedoch als Ausgangspunkt für nachfolgende Studien eingesetzt werden, die das Bild der Wirkung von THC auf die Fahrtüchtigkeit schließlich vervollständigen. 


\section{REFERENCES}

Abel EL (1971) Effects of marijuana on the solution of anagrams, memory and appetite. Nature, 231, 260-261.

Abel EL (1977) The relationship between cannabis and violence: A review. Psychological Bulletin, 84, 193-211.

Abel EL (1979) A Comprehensive Guide to the Cannabis Literature. Greenwood Press, Westport, $\mathrm{CN}$.

Abood ME and Martin BR (1992) Neurobiology of marijuana abuse. Trends in Pharmacological Sciences, 13, 201-207.

Agurell S, Halldin M, Lindgren JE, Ohlsson A, Widman M, Gillespie H and Hollister L (1986) Pharmacokinetics and metabolism of $\Delta^{1}$-tetrahydrocannabinol and other cannabinoids with emphasis on man. Pharmacological Reviezes, 38, 21-43.

Alvarez FJ, Prada R and Del Rio MC (1991) "Illegal" drugs and driving in Spain. Medical Science Research, 19, 491-492.

Alvarez FJ, Queipo D, Del Rio MC and Garcia MC (1992) Patterns of drug use in Castille and Leon (Spain). British Journal of Addiction, 87, 1153-1160.

Andréasson S, Allebeck P, Engström A and Rydberg U (1987) Cannabis and schizophrenia: A longitudinal study of Swedish conscripts. Lancet, 2, 1483-1485.

Astley SJ and Little RE (1990) Maternal marijuana use during lactation and infant development at one year. Neurotoxicology and Teratology, 12, 161-168.

Attwood DA, Williams RD, Bowser JS, McBurney LJ and Frecker RC (1981) The Effects of Moderate Levels of Alcobol and Marijuana, Alone and in Combination on Closed-Course Driving Performance. Defence and civil institute of environmental medicine (81-RSU-17), Downsview, Ontario.

Azorlosa JL, Heishman SJ, Stitzer ML and Mahaffey JM (1992) Marijuana smoking: Effect of varying $\Delta^{9}$-tetrahydrocannabinol content and number of puffs. Journal of Pharmacology and Experimental Therapeutics, 261, 114-122.

Baddely AD and Hitch GJ (1974) Working memory. In: The Psychology of Learning and Motivation. Vol. 8 (Ed GM Bower), pp. 123-175. Academic Press, New York.

Barnett G, Licko V and Thompson T (1985) Behavioral pharmacokinetics of marijuana. Psychopharmacology, 85, 51-56.

Bech P, Rafaelsen L and Rafaelsen OJ (1973) Cannabis and alcohol: Effects on Estimation of Time and Distance. Psychopharmacologia, 32, 373-381.

Belgrave BE, Bird KD, Chesher GB, Jackson DM, Lubbe KE, Starmer GA and Teo RKC (1979) The effect of cannabidiol, alone and in combination with ethanol, on human performance. Psychopharmacology, 64, 243-246. 
Benowitz NL and Jones RT (1981) Cardiovascular and metabolic considerations in prolonged cannabinoid administration in man. Journal of Clinical Pharmacology, 21, 214S-223S.

Block RI, Farinpour R and Schlechte JA (1991) Effects of chronic marijuana use on testosterone, luteinizing hormone, follicle stimulating hormone, prolactin and cortisol in men and women. Drug and Alcohol Dependence, 28, 121-128.

Block RI, Farinpour R and Braverman K (1992) Acute effects of marijuana on cognition: Relationships to chronic effects and smoking techniques. Pharmacology, Biochemistry and Behavior, 43, 907-917.

Bond A and Lader M (1974) The use of analogue scales in rating subjective feelings. British Journal of Medical Psychology, 47, 211-218.

Borg J, Gershon S and Alpert M (1975) Dose effects of smoked marihuana on human cognitive and motor functions. Psychopharmacologia, 42, 211-218.

Borkenstein RF, Crowther RF, Schumate RP, Ziel WB and Zylman R (1974) The Role of the Drinking Driver in Traffic Accidents (The Grand Rapids Study). Blutalkohol, 11(Suppl 1), 1-131.

Bouaboula M, Rinaldi M, Carayon P, Carillon C, Delpech B, Shire D, Le Fur G and Casellas P (1993) Cannabinoid-receptor expression in human leukocytes. European Journal of Biochemistry, 214, 173-180.

Brookhuis K, Volkerts E and O'Hanlon J (1987) The effects of some anxiolytics on car-following performance in real traffic. In: Alcohol, Drugs and Traffic Safety. Proceedings of the 10th International Conference on Alcohol, Drugs and Traffic Safety, September 9-12, 1986, Amsterdam, The Netherlands (Eds PC Noordzij and R Roszbach), pp. 223-226. Excerpta Medica, Amsterdam.

Bromberg W (1934) Marihuana intoxication: A clinical study of Cannabis sativa intoxication. American Journal of Psychiatry, 91, 303-330.

Budd RD, Muto JJ and Wong JK (1989) Drugs of abuse found in fatally injured drivers in Los Angeles County. Drug and Alcohol Dependence, 23, 153-158.

Burns M (1981) Marijuana Effects on Driving: A Survey of the Perceptions, Attitudes and Practices of High School Students. Southern California Research Institute, Los Angeles, CA.

Camí J, Guerra D, Ugena B, Segura J and De La Torre, R (1991) Effect of subject expectancy on the THC intoxication and disposition from smoked hashish cigarettes. Pharmacology, Biochemistry and Behavior, 40, 115-119.

Campbell I (1976) The amotivational syndrome and cannabis use with emphasis on the Canadian scene. Annals of the New York Academy of Sciences, 282, 33-36.

Campbell RL and Svenson LW (1992) Drug use among university undergraduate students. Psychological Reports, 70, 1039-1042.

Cappell HD and Pliner PL (1973) Volitional control of marijuana intoxication: A study of the ability to "come down" on command. Journal of Abnormal Psychology, 82, 428-434.

Cappell $\mathrm{H}$ and Pliner P (1974) Regulation of the self-administration of marihuana by psychological and pharmacological variables. Psychopharmacologia, 40, 65-76.

Cappell H, Kuchar E and Webster CD (1973) Some correlates of marihuana self-administration in man: A study of titration of intake as a function of drug potency. Psychopharmacologia, $29,177-184$.

Capriotti RM, Foltin RW, Brady JV and Fischman MW (1988) Effects of marijuana on the task-elicited physiological response. Drug and Alcohol Dependence, 21, 183-187.

Casswell S (1979) Cannabis and Alcohol: Effects on closed course driving behaviour. In: Seventh International Conference on Alcohol, Drugs and Traffic Safety. Proceedings. Melbourne 23-28 January 1977 (Ed I Johnston). Australian Government Publishing Service, Canberra. 
Casswell S and Marks D (1973) Cannabis and temporal disintegration in experienced and naive subjects. Science, 179, 803-805.

Chait LD (1989) Delta-9-tetrahydrocannabinol content and human marijuana self-administration. Psychopharmacology, 98, 51-55.

Chait LD (1990) Subjective and behavioral effects of marijuana the morning after smoking. Psychopharmacology, 100, 328-333.

Chait LD and Perry JL (1992) Factors influencing self-administration of, and subjective response to, placebo marijuana. Behavioural Pharmacology, 3, 545-552.

Chait LD, Fischman MW and Schuster CR (1985) 'Hangover' effects the morning after marijuana smoking. Drug and Alcohol Dependence, 15, 229-238.

Chang AE, Shiling DJ, Stillman RC, Goldberg N, Seipp C, Barofsky I, Simon R and Rosenberg $S$ (1979) Delta-9-tetrahydrocannabinol as an antiemetic in cancer patients receiving high-dose methotrexate: A prospective, randomized evaluation. Annals of Internal Medicine, 92, 819-824.

Chaudhury S, Augustine M and Chandra S (1989) Cannabis psychosis: Two case reports and a review. Journal of Personality and Clinical Studies, 5, 82-84.

Chaudry HR, Moss HB, Bashir A and Suliman T (1991) Cannabis psychosis following bhang ingestion. British Journal of Addiction, 86, 1075-1081.

Chesher GB (1986) The effects of alcohol and marijuana in combination: A review. Alcobol, Drugs and Driving: Abstracts and Reviews, 2, 105-120.

Chesher GB and Starmer GA (1983) Cannabis and Human Performance Skills. Drug and Alcohol Authority Research, Grant Report Series, Sydney, Australia, NSW.

Chesher GB, Dauncy H, Crawford J and Horn K (1986) The interaction between alcohol and marijuana: A dose dependent study of the effects on human moods and performance skills. Federal Office of Road Safety, Australia.

Chiang CW and Barnett G (1984) Marijuana effect and delta-9-tetrahydrocannabinol plasma level. Clinical Pharmacology Therapeutics, 36, 234-238.

Chopra G and Smith J (1974) Psychotic reactions following cannabis use in East Indians. Archives of General Psychiatry, 30, 24-27.

Cimbura G, Warren RA, Bennett RC, Lucas DM, and Simpson HM (1980) Drugs detected in fatally injured drivers and pedestrians in the Province of Ontario. Traffic Injury Research Foundation of Canada, Ottawa.

Cimbura G, Lucas DM, Bennett RC, Warren RA and Simpson HM (1982) Incidence and toxicological aspects of drugs detected in 484 fatally injured drivers and pedestrians in Ontario. Journal of Forensic Sciences, 27, 855-867.

Cimbura G, Lucas DM, Bennett RC and Donelson AC (1990) Incidence and toxicological aspects of cannabis and ethanol detected in 1394 fatally injured drivers and pedestrians in Ontario (1982-1984) Journal of Forensic Sciences, 35, 1035-1041.

Clapp JF, Wesley M, Cooke R, Pekala R and Holstein C (1987) The effects of marijuana smoke on gas exchange in ovine pregnancy. Alcohol and Drug Research, 7, 85-92.

Clark LD, Hughes R and Nakashima EF (1970) Behavioral effects of marihuana: Experimental studies. Archives of General Psychiatry, 23, 193-198.

Co BT, Goodwin DW, Gado M, Mikhael M and Hill SY (1977) Absence of cerebral atropy in chronic cannabis users: Evaluation by computerized transaxial tomography. Journal of the American Medical Association, 237, 1229-1230.

Cochetto DM, Owens SM, Perez-Reyes M, DiGuiseppi S and Miller LL (1981) Relationship between plasma delta-9-tetrahydrocannabinol concentration and pharmacological effects in man. Psychopharmacology, 75, 158-164. 
Coggins WG (1976) The Costa Rica cannabis project: An interim report on the medical aspects. In: Pharmacology of Marihuana (Eds MC Braude and S Szara), pp. 667-671. Raven Press, New York.

Cohen H (1975) Drugs, Druggebruikers and Drug-Scene. Samson, Alphen a/d Rijn.

Compton DR, Dewey WL and Martin BR (1990) Cannabis dependence and tolerance production. Advances in Alcobol and Substance Abuse, 9, 129-147.

Consroe R, Jones B and Laird H (1976) Interactions of delta-9-tetrahydrocannabinol with other pharmacological agents. Annals of the New York Academy of Sciences, 282, 198-211.

Consroe P, Sandyk R and Snider SR (1986) Open label evaluation of cannabidiol in dystonic movement disorders. International Journal of Neuroscience, 30, 277-282.

Cook E (1986) Analytical methodology for $\Delta^{9}$-tetrahydrocannabinol and its metabolites. Alcohol, Drugs and Driving: Abstracts and Reviews, 2, 79-92.

Council on Scientific Affairs (1985) Alcohol and the driver. Journal of the American Medical Association, 255, 522-527.

Crancer AJ, Dille JM, Delay JC, Wallace JE and Haken M (1969) Comparison of the effects of marijuana and alcohol on simulated driving performance. Science, 164, 851-854.

Dackis CA, Pottash ALC, Annitto W and Gold MS (1982) Persistence of urinary marijuana levels after supervised abstinence. American Journal of Psychiatry, 139, 1196-1198.

Daldrup T, Reudenbach G and Kimm K (1987) Cannabis und Alkohol im Strassenverkehr. Blutalkohol, 24, 144-156.

Darley CF, Tinklenberg JR, Roth WT and Atkinson RC (1974) The nature of storage deficits and state-dependent retrieval under marihuana. Psychopharmacologia, 37, 139-149.

Davis KH, McDaniel IA, Cadwell LW and Moody PL (1984) Some smoking characteristics of marijuana cigarettes. In: The Cannabinoids: Chemical, Pharmacologic and Therapeutic Aspects (Eds S Agurell, WL Dewey and RE Willette), pp. 97-1019. Academic Press, New York.

Davison GC and Neale JM (1978). Abnormal Psychology: An experimental approach. Wiley, New York.

Day N, Cornelius M, Goldschmidt L, Richardson G, Robles N, and Taylor P (1992) The effects of prenatal tobacco and marijuana use on offspring growth from birth through 3 years of age. Neurotoxicology and Teratology, 14, 407-414.

De Gier JJ (1979) A subjective measurement of the influence of ethyl/alcohol in moderate doses on real driving performances. Blutalkohol, 16, 363-370.

De Gier JJ, 't Hart BJ, Nelemans FA and Bergman H (1981) Psychomotor performance and real driving performance of outpatients receiving diazepam. Psychopharmacology, 73, 340-347.

Devane WA, Dysarz FA, Johnson MR, Melvin LS and Howlett AC (1988) Determination and characterization of a cannabinoid receptor in rat brain. Molecular Pharmacology, 34, 605-613.

Devane WA, Hanus L, Breuer A, Pertwee RG, Stevenson LA, Griffin G, Gibson D, Mandelbaum A, Etinger A and Mechoulam R (1992) Isolation and structure of a brain constituent that binds to the cannabinoid receptor. Science, 258, 1946-1949.

Dewey WL (1986) Cannabinoid pharmacology. Pharmacological Reviews, 38, 151-178.

Dewey WL, Martin BR and May EL (1984) Cannabinoid stereoisomers: Pharmacological effects. In: Handbook of Stereoisomers: Drugs in Psychopharmacology (Ed DF Smith), pp. 317-326. CRC Press, Boca Raton, FL.

De Zwart WM, Mensink C and Kuipers SBM (1993) Roken, Drinken, Druggebruik en Gokken onder Scholieren vanaf 10 jaar: Kerngegevens van het 3e Peilstations-Onderzoek naar Riskant Middelengebruik. Netherlands Institute on Alcohol and Drugs, Utrecht. 
Donelson AC, Cimbura G, Bennett RC and Lucas DM (1985) The Ontario monitoring project: Cannabis and alcohol use among drivers and pedestrians fatally injured in motor vehicle accidents from March 1982 through July 1984. Traffic Injury Research Foundation of Canada, Ottawa.

Dornbush and Kokkevi (1976) The acute effects of various cannabis substances on cognitive, perceptual and motor performance in very long term hashish users. In: Pharmacology of Maribuana (Eds MC Braude and S Szara), pp. 421-427. Raven Press, New York.

Dott AB (1972) Effect of Marijuana on Risk Acceptance in a Simulated Passing Task. Report ICRL-RR-71-3 DHEW Publication No. HSM-72-10010, US Public Health Service, Washington, DC.

Durnin JVGA and Womersley J (1974) Body fat assessed from total body density and its estimation from skinfold thickness: Measurements on 481 men and women aged from 16 to 72 years. British Journal of Nutrition, 32, 77-97.

Ellickson PL, Hays RD and Bell RM (1992) Stepping through the drug use sequence: Longitudinal scalogram analysis of initiation and regular use. Journal of Abnormal Psychology, $101,441-451$.

Ellingstad VS, McFarling LH and Struckman DL (1973) Alcohol, marijuana and risk taking. Vermillion Human Factors Laboratory, South Dakota University.

Ellis GM, Mann MA, Judson BA, Schramm NT and Tashchian A (1985) Excretion patterns of cannabinoid metabolites after last use in a group of chronic users. Clinical Pharmacology and Therapeutics, 38, 572-578.

Fabian WD and Fishkin SM (1981) A replicated study of self-reported changes in psychological absorption with marijuana intoxication. Journal of Abnormal Psychology, 90, 546-553.

Farre-Albaladejo M (1989) Complicaciones neurologicas de las toxicomanias. Aspectos generales. Complicaciones por "cannabis", drogas de diseno y sustancias volatiles. Archivos de Neurobiologia, 52(Suppl 1), 143-148.

Fehr K and Kalant H (1983) Long-term effects of cannabis on cerebral function: A review of the clinical and experimental literature. In: Cannabis and Health Hazards. Proceedings of an ARF/WHO Scientific Meeting on Adverse Health and Behavioral Consequences of Cannabis Use (Eds $\mathrm{K}$ Fehr and $\mathrm{H}$ Kalant), Toronto.

Ferrara SD and Rozza M (1985) Alcohol, drugs and road accidents: Epidemiological study in north-east Italy. In: Alcohol, Drugs and Traffic Safety. Proceedings of the 9th International Conference on Alcohol, Drugs and Traffic Safety, November 1983, San Juan, Puerto Rico (Eds S Kaye and G Meier), pp. 469-485. Report No. DOT-HS-806-814, United States Department of Transportation, Washington, DC.

Fischer J and Täschner K-L (1991) Flashback nach Cannabiskonsum: Eine Ubersicht. Fortschritte der Neurologie, Psychiatrie, 59, 437-446.

Foltin RW, Brady JV and Fischman MW (1986) Behavioral analysis of marijuana effects on food intake in humans. Pharmacology, Biochemistry and Behavior, 25, 577-582.

Foltin RW, Fischman MW and Byrne MF (1988) Effects of smoked marijuana on food intake and body weight of humans living in a residential laboratory. Appetite, 11, 1-14.

Fraisse P (1963) The Psychology of Time. Harper and Row, New York.

Frank DA, Bauchner H, Parker S, Huber AM, Kyei-Aboagye K, Cabral H and Zuckerman B (1990) Neonatal body proportionality and body composition after in utero exposure to cocaine and marijuana. Journal of Pediatrics, 117, 622-626.

Fried PA (1989a) Cigarettes and marijuana: Are there measurable long-term neurobehavioral teratogenic effects? Neurotoxicology, 10, 577-583.

Fried PA (1989b) Cigarettes and marijuana: Are there measurable long-term neurobehavioral teratogenic effects? Neurotoxicology, 10, 577-583. 
Fried PA and Makin JE (1987) Neonatal behavioural correlates of prenatal exposure to marihuana, cigarettes and alcohol in a low risk population. Neurotoxicology and Teratology, 9, 1-7.

Fried PA and Watkinson B (1990) 36- and 48-month neurobehavioral follow-up of children prenatally exposed to marijuana, cigarettes, and alcohol. Joumal of Developmental and Behavioral Pediatrics, 11, 49-58.

Fried PA, Buckingham M, and Von Kulmiz P (1983) Marijuana use during pregnancy and perinatal risk factors. American Journal of Obstetrics and Gynecology, 144, 922-924.

Fried PA, Watkinson B, and Willan A (1984) Marijuana use during pregnancy and decreased length of gestation. American Journal of Obstetrics and Gynecology, 150, 23-27.

Fried PA, Watkinson B, and Gray R (1992) A follow-up study of attentional behavior in 6-year-old children exposed prenatally to marihuana, cigarettes, and alcohol. Neurotoxicology and Teratology, 14, 299-311.

Gaoni Y and Mechoulam R (1964) Isolation, structure and partial synthesis of an active constituent of hashish. Journal of the American Chemical Society, 86, 1646-1647.

Gardner EL and Lowinson JH (1991) Marijuana's interaction with brain reward systems: Update 1991. Pharmacology, Biochemistry and Behavior, 40, 571-580.

Gardner EL, Paredes W, Smith D, Donner A, Milling C and Cohen D (1988) Facilitation of brain stimulation reward by D-sup-9-tetrahydrocannabinol. Psychopharmacology, 96, 142-144.

Garriott JC, Di Maio VJM and Rodriguez RG (1986) Detection of cannabinoids in homicide victims and motor vehicle fatalities. Journal of Forensic Sciences, 31, 1274-1282.

Gérard CM, Mollereau C, Vassart G and Parmentier M (1991) Molecular cloning of a human cannabinoid receptor which is also expressed in testis. Biochemical Journal, 279, 129-134.

Gieringer DH (1988) Marijuana, driving, and accident safety. Journal of Psychoactive Drugs, 20, 93-101.

Gray JA (1982) The Neuropsychology of Anxiety: An Enquiry into the Functions of the Septo-Hippocampal System. Clarendon Press, Oxford and Oxford University Press, New York.

Grinspoon L and Bakalar JB (1992) Marihuana. In: Substance Abuse. A Comprehensive Textbook (Eds JH Lowinson, P Ruiz and RB Millman), Pp. 236-246. Raven Press, New York.

Grinspoon L and Bakalar JB (1993) Maribuana, the Forbidden Medicine. Yale University Press, New Haven.

Gust SW and Pickens RW (1982) Does nicotine yield affect puff volume? Clinical Pharmacology and Therapeutics, 36, 418-422.

Hansteen RW, Miller RD, Lonero L, Reid LD and Jones B (1976) Effects of cannabis and alcohol on automobile driving and psychomotor tracking. Annals of the New York Academy of Sciences, 282, 240-256.

Harvey DJ (1984) Chemistry, metabolism, and pharmacokinetics of the cannabinoids. In: Marihuana in Science and Medicine (Ed GG Nahas), pp. 37-107. Raven Press, New York.

Hayes JS, Lampart R, Dreher MC, and Morgan L (1991) Five-year follow-up of rural Jamaican children whose mothers used marijuana during pregnancy. West Indian Medical Journal, 40, 120-123.

Heaton RK and Victor RG (1976) Personality characteristics associated with psychedelic flashbacks in natural and experimental settings. Journal of Abnormal Psychology, 85, 83-90.

Heishman SJ, Stitzer ML and Yingling JE (1989) Effects of tetrahydrocannabinol content on marijuana smoking behavior, subjective reports, and performance. Pharmacology, Biochemistry \& Behavior, 34, 173-179. 
Heishman SJ, Huestis MA, Henningfield JE and Cone EJ (1990) Acute and residual effects of marijuana: Profiles of plasma THC levels, physiological, subjective, and performance measures. Pharmacology, Biochemistry and Behavior, 37, 561-565.

Hembree III WC, Nahas GG, Zeindenberg P and Huang HFS (1979) Changes in human spermatozoa associated with high dose marihuana smoking. In: Marihuana. Biological Effects (Eds GG Nahas and WDM Paton), pp. 429-440. Pergamon Press, Oxford.

Henderson RL, Tennant FS and Guerney R (1972) Respiratory manifestations of hashish smoking. Archives of Otolaryngology, 95, 248-251.

Herkenham M, Lynn AB, Little MD, Johnson MR, Melvin LS, De Costa BR and Rice KC (1990) Cannabinoid receptor localization in brain. Proceedings of the National Academy of Sciences of the U.S.A., 87, 1932-1936.

Herkenham M, Lynn AB, De Costa BR and Richfield EK (1991) Neuronal localization of cannabinoid receptors in the basal ganglia of the rat. Brain Research, 547, 267-274.

Herning RI, Jones RT, Bachman J and Mines AH (1981) Puff volume increases when low-nicotine cigarettes are smoked. British Medical Journal, 283, 187-193.

Herning RI, Hooker WD and Jones RT (1986) Tetrahydrocannabinol content and differences in marijuana smoking behavior. Psychopharmacology, 90, 160-162.

Hicks RE, Gualtieri CT, Mayo JP and Perez-Reyes M (1984) Cannabis, atropine, and temporal information processing. Neuropsychobiology, 12, 229-237.

Himmelstein JL (1983) The Strange Career of Maribuana: Politics and Ideology of Drug Control in America. Greenwood Press, Westport, CN.

Hingson R, Alpert J, Day N, Dooling E, Kayne H, Morelock S, Oppenheimer E and Zuckerman B (1982) Effects of maternal drinking and marijuana use on fetal growth and development. Pediatrics, 70, 539-546.

Hockey GRJ (1986) A state control theory of adaptation of stress and individual differences in stress management. In: Energetics and Human Information Processing (Eds GRJ Hockey, AWK Gaillard and MGH Coles), pp. 285-298. Martinus Nijhoff Publishers, Dordrecht.

Hollister LE (1971a) Marihuana in man: Three years later. Science, 172, 21-29.

Hollister LE (1971b) Hunger and appetite after single doses of marihuana, alcohol, and dextroamphetamine. Clinical Pharmacology and Therapeutics, 12, 44-49.

Hollister LE (1973) Cannabidiol and cannabinol in man. Experientia, 29, 825-826.

Hollister LE (1986) Health aspects of cannabis. Pharmacological Reviews, 38, 1-20.

Hollister LE (1988) Cannabis - 1988. Acta Psychiatrica Scandinavica, 78(Suppl 345), 108-118.

Hollister LE and Gillespie HK (1970) Marihuana, ethanol, and dextroamphetamine. Archives of General Psychiatry, 23, 199-207.

Hollister LE and Kanter SL (1980) Laboratory verification of "heavy" and "light" users of cannabis. Drug and Alcohol Dependence, 5, 151-152.

Hollister LE, Overall JE and Gerber ML (1975) Marihuana and setting. Archives of General Psychiatry, 32, 798-803.

Hollister LE, Gillespie H, Ohlsson A, Lindgren JE, Wahlén A and Agurell S (1981). Do plasma concentrations of $\Delta^{9}$-tetrahydrocannabinol reflect the degree of intoxication? Journal of Clinical Pharmacolology, 21, 171S-177S.

Hooker WD and Jones RT (1987) Increased susceptibility to memory intrusions and the Stroop interference effect during acute marijuana intoxication. Psychopharmacology, 91, 20-24.

Howlett AC (1985) Cannabinoid inhibition of adenylate cyclase. Biochemistry of the response in neuroblastoma cell membranes. Molecular Pharmacology, 27, 429-436.

Howlett AC (1987) Cannabinoid inhibition of adenylate cyclase: Relative activities of marihuana constituents and metabolites. Neuropharmacology, 26, 507-512. 
Howlett AC, Johnson MR, Melvin LS and Milne GM (1988) Nonclassical cannabinoid analgetics inhibit adenylate cyclase: Development of a cannabinoid receptor model. Molecular Pharmacology, 33, 297-302.

Howlett AC, Bidaut-Russell M, Devane WA, Melvin LS, Johnson MR and Herkenham M (1990) The cannabinoid receptor: Biochemical, anatomical and behavioral characterization. Trends in Neurosciences, 13, 420-423.

Huestis MA, Henningfield JE and Cone EJ (1992) Blood cannabinoids. I. Absorption of THC and formation of 11-OH-THC and THCCOOH during and after smoking marijuana. Journal of Analytical Toxicology, 16, 276-282.

Hunt CA and Jones RT (1980) Tolerance and disposition of tetrahydrocannabinol in man. Journal of Pharmacology and Experimental Therapeutics, 215, 35.

Indanpaan-Heikkila J, Fritchie EG, Englert LF, Ho TB and McIsaac WM (1969) Placental transfer of tritiated 1-delta-9-tetrahydrocannabinol. The New England Journal of Medicine, $281,330$.

Jaffe JH (1990) Drug addiction and drug abuse. In: Goodman and Gilman's The Pharmacological Basis of Therapeutics (Eds AG Goodman Gilman, ThW Rall, AS Nies and P Taylor), pp. 284-324. Pergamon Press, New York.

Jessor R, Donovan JE and Costa F (1986) Psychosocial correlates of marijuana use in adolescence and young adulthood: The past as prologue. Alcohol, Drugs and Driving: Abstracts and Reviews, 2, 31-50.

Jex HR, McDonnell JD and Phatak AV (1966) A "critical" tracking task for manual control research. Institute of Electrical and Electronic Engineers Transactions on Human Factors in Electronics, 7, 138-145.

Johansson E, Agurell S, Hollister LE and Halldin MM (1988) Prolonged apparent half-life of $\Delta^{1}$-tetrahydrocannabinol in plasma of chronic marijuana users. Journal of Pharmacy and Pharmacology, 40, 374-375.

Johansson E, Halldin MM, Agurell S, Hollister LE and Gillespie HK (1989a) Terminal elimination plasma half-life of $\Delta^{1}$-tetrahydrocannabinol ( $\Delta^{1}-$ THC) in heavy users of marijuana. European Journal of Clinical Pharmacology, 37, 273-277.

Johansson E, Norén K, Sjövall J and Halldin MM (1989b) Determination of $\Delta^{1}$-tetrahydrocannabinol in human fat biopsies by gas chromatography-mass spectrometry. Biomedical Chromatography, 3, 35-38.

Johnson BD (1973) Maribuana Users and Drug Subcultures. Wiley, New York.

Johnson BS, Smith BL and Taylor P (1988) Cannabis and schizophrenia. Lancet, 1, 592-593. Johnson MR and Melvin LS (1986) The discovery of nonclassical cannabinoid analgetics. In: Cannabinoids as Therapeutic Agents (Ed R Mechoulam), Pp. 121-145. CRC Press, Boca Raton, FL.

Johnston LD, O'Malley PM and Bachman JG (1992) Smoking, Drinking, and Illicit Drug Use among American Secondary School Students, College Students, and Young Adults, 1975-1991. Institute for Social Research, University of Michigan, USA.

Jones MH (1978) Driver Performance Measures for the Safe Performance Curriculum. Traffic Safety Center, Institute of Safety and Systems Management, University of South California, Los Angeles, CA (DOT HS 803 461).

Jones RT (1971) Marihuana-induced 'high': Influence of expectation, setting and previous drug experience. Pharmacological Reviews, 23, 359-369.

Jones RT, Benowitz NL and Herning RI (1981) Clinical relevance of cannabis tolerance and dependence. Journal of Clinical Pharmacology, 21, 143S-152S. 
Joscelyn KB, Donelson AC, Jones RK, McNair JW and Ruschmann PA (1980) Drugs and Highway Safety 1980. Highway Safety Research Institute, The university of Michigan, Michigan (DOT HS 805 461).

Kahneman D (1973) Attention and Effort. Prentice-Hall, New Jersey.

Kandel D (1984) Substance abuse by adolescents in Israel and France: A cross-cultural perspective. Public Health Reports, 99, 277-283.

Kapteyn TS, Bles W, Njiokiktjein ChJ, Rodde L, Massen CH and Mol JMF (1983) Standardization in platform stabilometry being part of posturography. Agressologie, 24, 321-326.

Karniol IG and Carlini EA (1973) Pharmacological interaction between cannabidiol and $\Delta^{9}$-tetrahydrocannabinol. Psychopharmacologia, 33, 53-70.

Kelly TH, Foltin RW, Emurian CS and Fischman MW (1990) Multidimensional behavioral effects of marijuana. Progress in Neuro-Psychopharmacology and Biological Psychiatry, 14, 885-902.

Klonoff H (1974) Marijuana and driving in real-life situations. Science, 186, 317-323.

Kolodny RC, Masters WH, Kolodny RM and Toro G (1974) Depression of plasma testosterone levels after chronic intensive marihuana use. The New England Journal of Medicine, 290, 872-874.

Leirer VO, Yesavage JA and Morrow DG (1989) Marijuana, aging, and task difficulty effects on pilot performance. Aviation, Space, and Environmental Medicine, 60, 1145-1152.

Leirer VO, Yesavage JA and Morrow DG (1991) Marijuana carry-over effects on aircraft pilot performance. Aviation, Space, and Environmental Medicine, 62, 221-227.

León-Carrión J (1990) Mental performance in long-term heavy cannabis use: A preliminary report. Psychological Reports, 67, 947-952.

Lindenbaum GA, Carroll SF, Daskal I, and Kapusnick R (1989) Patterns of alcohol and drug abuse in an urban trauma center: The increasing role of cocaine abuse. The Journal of Trauma, 29, 1654-1658.

Lindgren JE, Ohlsson A, Agurell S, Hollister L and Gillespie H (1981) Clinical effects and plasma levels of delta-nine-tetrahydrocannabinol in heavy and light users of cannabis. Psychopharmacology, 74, 208-212.

Louwerens JW, Gloerich ABM, De Vries G, Brookhuis KA and O'Hanlon JF (1985) De Invloed van Verschillende Bloedalcoholspiegels op Objectief Meetbare Aspekten van Feitelijk Rijgedrag. Technical Report No. VK 85-03, Traffic Research Centre, University of Groningen.

Louwerens JW, Gloerich ABM, De Vries G, Brookhuis KA and O'Hanlon JF (1987) The relationship between drivers' blood alcohol concentration (BAC) and actual driving performance during high speed travel. In: Alcohol, Drugs and Traffic Safety. Proceedings of the 10th International Conference on Alcohol, Drugs and Traffic Safety, September 9-12, 1986, Amsterdam, The Netherlands (Eds PC Noordzij and R Roszbach), Pp. 183-192. Excerpta Medica, Amsterdam.

Marks DF and MacAvoy MG (1989) Divided attention performance in cannabis users and non-users following alcohol and cannabis separately and in combination. Psychopharmacology, 99, 397-401.

Marks DF and Pow GM (1989) Cannabis and human social behaviour. Human Psychopharmacology, 4, 283-290.

Martin BR (1986) Cellular effects of cannabinoids. Pharmacological Reviews, 38, 45-74.

Mason AP and McBay AJ (1984) Ethanol, Marijuana, and other drug use in 600 drivers killed in single-vehicle crashes in North Carolina, 1978-1981. Joumal of Forensic Sciences, 29, 987-1026. 
Mathers DC and Ghodse AHC (1992) Cannabis and psychotic illness. British Journal of Psychiatry, 161, 648-653.

Mathew RJ, Wilson WH and Tant SR (1989) Acute changes in cerebral blood flow associated with marijuana smoking. Acta Psycbiatrica Scandinavica, 79, 118-128.

Mathew RJ, Wilson WH, Humphreys D, Lowe JV and Weithe KE (1993) Depersonalization after marijuana smoking. Biological Psychiatry, 33, 431-441.

Matsuda LA, Lolait SJ, Brownstein MJ, Young AC and Bonner TI (1990) Structure of a cannabinoid receptor and functional expression of the cloned cDNA. Nature, 346, 561-564.

Maykut MO (1985) Health consequences of acute and chronic marihuana use. Progress in Neuro-Psychopharmacology and Biological Psychiatry, 9, 209-238.

McBay A (1977) Marijuana. In: Drugs and Driving, pp. 91-98. National Institute on Drug Abuse Research Monograph Series 11.

McLean S, Parsons RS, Chesterman RB, Dineen R, Johnson MG and Davies NW (1987) Drugs, alcohol and road accidents in Tasmania. The Medical Journal of Australia, 147, 6-11.

Meinck H-M, Schönle PW and Conrad B (1989) Effect of cannabinoids on spasticity and ataxia in multiple sclerosis. Journal of Neurology, 236, 120-122.

Melges FT, Tinklenberg JR, Hollister LE and Gillespie HK (1970a) Temporal disintegration and depersonalization during marihuana intoxication. Archives of General Psychiatry, 23, 204-210.

Melges FT, Tinklenberg JR, Hollister LE and Gillespie HK (1970b) Marihuana and temporal disintegration. Science, 168, 1118-1120.

Mendhiratta SS, Wig NN and Verma SK (1978) Some psychological correlates of long-term heavy cannabis users. British Journal of Psychiatry, 132, 482-486.

Mendhiratta SS, Varma VK, Dang R, Malhotra AK, Das K and Nehra R (1988) Cannabis and cognitive functions: A re-evaluation study. British Journal of Addiction, 83, 749-753.

Merrit JC, Crawford WJ, Alexander PC, Anduze AL and Gelbart SS (1980) Effect of marihuana on intraocular and blood pressure in glaucoma. Ophthamology, 87, 222-228.

Meyer RE, Pillard RC, Shapiro LM and Mirin SM (1971) Administration of marijuana to heavy and casual marijuana users. American Joumal of Psychiatry, 128, 198-204.

Meyman TF and Zijlstra FRH (1986) The measurement of perceived effort. In: Contemporary Ergonomics (Ed DJ Oborne), pp. 242-247. Taylor and Francis, London.

Miller NS and Gold MS (1989) The diagnosis of marijuana (Cannabis) dependence. Journal of Substance Abuse Treatment, 6, 183-192.

Miller LL, Cornett TL, Brightwell DR, McFarland DJ, Drew WG and Wikler A (1977a). Marijuana: Effects on storage and retrieval of prose material. Psychopharmacology, 51, 311-316.

Miller LL, McFarland DJ, Cornett TL, Brightwell DR and Wikler A (1977b). Marihuana: Effects on free recall and subjective organization of pictures and words. Psychopharmacology, $55,257-262$.

Milner G (1977) Marijuana and driving hazards. The Medical Journal of Australia, 1, 208.

Milstein SL, MacCannell K, Karr G and Clark S (1975) Marijuana-produced impairments in coordination. Joumal of Nervous and Mental Disease, 161, 26-31.

Möller MR, Dörr G and Warth S (1992) Simultaneous quantitation of delta-9-tetrahydrocannabinol (THC) and 11-nor-9-carboxy-delta-9-tetrahydrocannabinol (THC-COOH) in serum by GC/MS using deuterated internal standards and its application to a smoking study and forensic cases. Journal of Forensic Sciences, 37, 969-983.

Morakinyo O (1983) Aversion therapy of cannabis dependence in Nigeria. Drug and Alcohol Dependence, 12, 287-293. 
Moskowitz H (1985) Marijuana and driving. Accident Analysis and Prevention, 17, 323-346. Moskowitz H, Sharma S and McGlothlin W (1972) Effect of marihuana upon peripheral vision as a function of the information processing demands in central vision. Perceptual and Motor Skills, 35, 875-882.

Moskowitz H, Hulbert S and McGlothlin W (1976) Marijuana: Effects on simulated driving performance. Accident Analysis and Prevention, 8, 45-50.

Moskowitz H, Sharma S and Ziedman K (1981) Duration of skills performance impairment. Proceedings of the 25th Conference of the American Association for Automotive Medicine, 87-96.

Mulder G (1980) The Heart of Mental Effort: Studies in the Cardiovascular Psychophysiology of Mental Work. Doctoral Dissertation, University of Groningen, Groningen, The Netherlands.

Mulder G and Mulder LJM (1981) Information processing and cardiovascular control. Psychophysiology, 14, 392-402.

Murray JB (1985) Marijuana's effects on human cognitive functions, psychomotor functions, and personality. The Journal of General Psychology, 113, 23-55.

Nahas G, Leger C, Tocque B and Hoellinger H (1981) The kinetics of cannabinoid distribution and storage with special reference to the brain and testis. Journal of Clinical Pharmacology, 21, 208S-214S.

National Commission on Marihuana and Drug Abuse (1972) Maribuana: A Signal of Misunderstanding. US Government Printing Office, Washington, DC.

Negrete J (1989) Cannabis and schizophrenia (editorial). British Journal of Addiction, 84, 349-351.

O'Hanlon JF, Haak TW, Blaauw GJ and Riemersma JBJ (1982) Diazepam impairs lateral position control in highway driving. Science, 217, 79-81.

O'Hanlon JF, Brookhuis KA, Louwerens JW and Volkerts ER (1986) Performance testing as part of drug registration. In: Drugs and Driving (Eds JF O'Hanlon and JJ de Gier), pp. 311-330. Taylor and Francis, London.

Ohlsson A, Lindgren JE, Wahlén A, Agurell S, Hollister LE and Gillespie HK (1980) Plasma delta-nine-tetrahydrocannabinol concentrations and clinical effects after oral and intravenous administration and smoking. Clinical Pharmacology and Therapeutics, 28, 409-416.

Overall JE and Rhoades HM (1987) Adjusting P values for multiple tests of significance. In: Psychopharmacology. The Third Generation of Progress (Ed HY Meltzer), pp. 1013-1018. Raven Press, New York.

Owens SM (1981) Marijuana Use among Drivers in Fatal Single-Vehicle Accidents. Ph.D. Dissertation, University of North Carolina, Chapel Hill, NC.

Peck RC, Biasotti A, Boland PN, Mallory C and Reeve V (1986) The effects of marijuana and alcohol on actual driving performance. Alcohol, Drugs and Driving: Abstracts and Reviews, 2, 135-154.

Pedersen JM (1992) Substance abuse among Greenlandic school children. Arctic Medical Research, 51, 67-71.

Perez-Reyes M, Timmons MC, Davis and Wall ME (1973) A comparison of the pharmacological activity in man of intravenously administered $\Delta^{9}$-tetrahydrocannabinol, cannabinol, and cannbidiol. Experientia, 29, 1368-1369.

Perez-Reyes M, Timmons MC and Wall ME (1974) Long-term use of marihuana and the development of tolerance or sensitivity to $\Delta^{9}$-tetrahydrocannabinol. Archives of General Psychiatry, 31, 89-91. 
Perez-Reyes M, Owens SM and Di Guiseppi S (1981) The clinical pharmacology and dynamics of marihuana cigarette smoking. Journal of Clinical Pharmacology, 21, 201S-207S.

Perez-Reyes M, Di Guiseppie S, Davis KH, Schindler VH and Cook CE (1982) Comparison of effects of marijuana cigarettes of three different potencies. Clinical Pharmacology and Therapeutics, 31, 617-624.

Perez-Reyes M, Hicks RE, Bumberry J, Jeffcoat AR and Cook CE (1988) Interaction between marihuana and ethanol: Effects on psychomotor performance. Alcoholism: Clinical and Experimental Research, 12, 268-276.

Petro DJ and Ellenberger C (1981) Treatment of human spasticity with $\Delta^{9}$-THC. Journal of Clinical Pharmacology, 21, 413S-416S.

Plasse TF, Gorter RW, Krasnow SH, Lane M, Shepard KV and Wadleigh RG (1991) Recent clinical experience with dronabinol. Pharmacology, Biochemistry and Behavior, 40, 695-700.

Plomp HN, Kuipers H and Van Oers ML (1990) Roken, Alcohol-en Drugsgebruik onder Scholieren vanaf 10 jaar. VU Uitgeverij, Amsterdam.

Pribram KH and McGuinness D (1975) Arousal, activation and effort in the control of attention. Psychological Review, 82, 116-146.

Rafaelsen OJ, Bech P, Christiansen J, Christup H, Nyboe J and Rafaelsen L (1973a). Cannabis and alcohol: Effects on simulated car driving. Science, 179, 920-923.

Rafaelsen L, Christup H, Bech P and Rafaelsen OJ (1973b) Effects of cannabis and alcohol on psychological tests. Nature, 242, 117-118.

Ramaekers JG, Uiterwijk MMC and O'Hanlon JF (1992a) Effects of loratadine and cetirizine on actual driving and psychometric test performance, and EEG during driving. European Journal of Clinical Pharmacology, 42, 363-369.

Ramaekers JG, Van Boxtel MPJ and O'Hanlon JF (1992b) A Study to Compare Acrivastine's and Terfenadine's Effects on Several Aspects of Driving Performance as a Function of Dose and Time after Dosing. Technical Report No. IGVG 92-25, Institute for Drugs, Safety and Behavior, University of Limburg, Maastricht, The Netherlands.

Randall RC (1990) Cancer Treatment and Marijuana Therapy. Galen Press, Washington, DC.

Rankin DW (1985) Epidemiological studies of alcohol and drug use by the youth of Australia. International Journal of the Addictions, 20, 1451-1461.

Razdan RK (1986) Structure-activity relationships in cannabinoids. Pharmacological Reviezes, $38,75-148$.

Reeve VC, Robertson WB, Grant J, Soares JR, Zimmerman EG, Gillespie HK and Hollister LE (1983) Hemolyzed blood and serum levels of $\Delta^{9}$-THC: Effects on the performance of roadside sobriety tests. Journal of Forensic Sciences, 28, 963-971.

Relman AS (1982) Marihuana and health. The New England Journal of Medicine, 306, 603-604. Riedel WJ, Schoenmakers EAJM and O'Hanlon JF (1989) Sedation and performance impairment with antihistamines. In: Management of Allergy in the 1990's (Ed Makaliner). Hans Huber, Toronto.

Riedel WJ, Ramaekers JG, Uiterwijk MMC and O'Hanlon JF (1990) Higher doses of terfenadine and loratadine; acute and subchronic effects on psychomotor and actual driving performance. Technical Report No. IGVG 90-08, Institute for Drugs, Safety and Behavior, University of Limburg, Maastricht, The Netherlands.

Robbe HWJ, Schoenmakers EAJM and O'Hanlon JF (1989) Paroxetine and Amitriptyline: Acute and Subchronic Effects on Psychomotor and Actual Driving Performance. Technical Report No. IGVG 89-04, Institute for Drugs, Safety and Behavior, University of Limburg, Maastricht, The Netherlands. 
Rossi AM, Kuehnle JC and Mendelson JH (1978) Marijuana and mood in human volunteers. Pharmacology, Biochemistry and Behavior, 8, 447-453.

Roth SH and Williams PJ (1979) The nonspecific membrane binding properties of $\Delta^{9}$ tetrahydrocannabinol and the effects of various solubilizers. Joumal of Pharmacy and Pharmacology, 31, 224-230.

Salemink CA (1984) De huidige stand van het internationale cannabis-onderzoek. Tijdschrift voor Alcohol, Drugs en Andere Psychotrope Stoffen, 10, 93-100.

Salzman C, Van der Kolk BA and Shader RI (1976) Marijuana and hostility in a small-group setting. American Journal of Psychiatry, 133, 1029-1033.

Schwin R, Hill SJ, Goodwin DW and Powell B (1974) Marijuana and critical flicker fusion. Journal of Nervous and Mental Disorders, 158, 142-144.

Sharma S and Moskowitz H (1973) Marijuana dose study of vigilance performance. Proceedings, 81st Annual Conference American Psychological Association (pp 1035-1036).

Sharma S and Moskowitz H (1974) Effects of two levels of attention demand on vigilance performance under marihuana. Perceptual and Motor Skills, 38, 967-970.

Sharma S and Moskowitz H (1975) Marihuana effects on a critical tracking task. Proceedings of the 19th Conference of the American Association for Automotive Medicine, 285-291.

Shedler J and Block J (1990) Adolescent drug use and psychological health: A longitudinal inquiry. American Psychologist, 45, 612-630.

Sherman MP, Campbell LA, Gong H Jr, Roth MD and Tashkin DP (1991) Antimicrobial and respiratory burst characteristics of pulmonary alveolar macrophages recovered from smokers of marijuana alone, smokers of tobacco alone, smokers of marijuana and tobacco, and nonsmokers. American Review of Respiratory Disease, 144, 1351-1356.

Shiffrin RM and Schneider W (1977) Controlled and automatic human information processing: II. Perceptual learning, automatic attending, and a general theory. Psychological Review, 84, 127-190.

Simpson HM (1986) Epidemiology of road accidents involving marijuana. Alcohol, Drugs and Driving: Abstracts and Reviews, 2, 15-30.

Sloan EP, Zalenski RJ, Smith RF, Sheaff CM, Chen EH, Keys NI, Crescenzo M, Barrett JA and Berman $E$ (1989) Toxicology screening in urban trauma patients: Drug prevalence and its relationship to trauma severity and management. The Journal of Trauma, 29, 1647-1653.

Small E and Cronquist A (1976) A practical and natural toxonomy for cannabis. Taxon, 25, 405-435.

Smiley AM (1986) Marijuana: On-road and driving simulator studies. Alcobol, Drugs and Driving: Abstracts and Reviews, 2, 121-134.

Smiley AM, Moskowitz H and Ziedman K (1981) Driving simulator studies of marijuana alone and in combination with alcohol. Proceedings of the 25th Conference of the American Association for Automotive Medicine, 107-116.

Smiley AM, Noy YI and Tostowaryk W (1987) The effects of marijuana alone and in combination with alcohol on driving performance. In: Alcobol, Drugs and Traffic Safety. Proceedings of the 10th International Conference on Alcohol, Drugs and Traffic Safecy, September 9-12, 1986, Amsterdam, The Netherlands (Eds PC Noordzij and R Roszbach), PP. 203-206. Excerpta Medica, Amsterdam.

Smith G, Wolynetz M, Davidson M and Poulton H (1975) Estimated blood alcobol concentrations of nighttime Canadian drivers. Traffic Injury Research Foundation (TIRF) of Canada, Ottawa.

Soderstrom CA, Trifillis AL, Shankar BS, Clark WE and Cowley RA (1988) Marijuana and alcohol use among 1023 trauma patients. Archives of Surgery, 123, 733-737. 
Solowij N, Michie PT and Fox AM (1991) Effects of long-term cannabis use on selective attention: An event-related potential study. Pharmacology, Biochemistry and Behavior, 40, 683-688.

Soueif MI (1975) Chronic cannabis takers: Some temperamental characteristics. Drug and Alcohol Dependence, 1, 125-154.

Stapleton JM, Guthrie S and Linnoila M (1986) Effects of alcohol and other psychotropic drugs on eye movements: Relevance to traffic safery. Journal of Studies on Alcohol, 47, 426-432.

Stefanis C, Boulougouris J and Liakos A (1976) Clinical and psychophysiological effects of cannabis in long-term users. In: Pharmacology of Marihuana (Eds MC Braude and S Szara), Pp. 659-665. Raven Press, New York.

Stein AC, Allen RW, Cook ML and Karl RL (1983) A Simulator Study of the Combined Effects of Alcobol and Marijuana on Driving Behavior. Report No. DOT HS 806 405, System Technology, Inc., Hawthorne, CA.

Tart C (1970) Marijuana intoxication: Common experiences. Nature, 226, 701-704.

Tashkin DP, Shapiro BJ, Lee YE and Harper CE (1976) Subacute effects of heavy marihuana smoking on pulmonary function in healthy men. The New England Journal of Medicine, 294, 125-129.

Tashkin DP, Gliederer F, Rose J, Chang P, Hui KK, Yu JL and Wu T-C (1991) Effects of varying marijuana smoking profile on deposition of tar and absorption of $\mathrm{CO}$ and delta-9-THC. Pharmacology, Biochemistry and Behavior, 40, 651-656.

Terhune KW (1982) The Role of Alcohol, Marijuana and Other Drugs in the Accidents of Injured Drivers. Technical Report to US Department of Transportation, Calspan Field Services, Inc.

Terhune KW, Ippolito CA, Hendricks DL, Michalovic JG, Bogema SC, Santinga P, Blomberg $\mathrm{R}$ and Dreusser DF (1992) The Incidence and Role of Drugs in Fatally Injured Drivers. Department of Transportation, Washington, DC.

Thornicroft G (1990) Cannabis and psychosis: Is there epidemiological evidence for an association? British Journal of Psychiatry, 157, 25-33.

Tinklenberg JR, Melges FT, Hollister LE and Gillespie HK (1970) Marijuana and immediate memory. Nature, 226, 1171-1172.

Tunving K (1985) Psychiatric effects of cannabis use. Acta Psychiatrica Scandinavica, 72, 209-217.

Tunving K, Thunlin SO, Risberg J and Warkentin S (1986) Regional cerebral blood flow in long-term heavy cannabis use. Psychiatry Research, 17, 15-21.

Turner CE, Elsohly MA and Boeren EG (1980) Constituents of cannabis sativa L. XVII. A review of the natural constituents. Journal of Natural Products, 43, 169-234.

Valzelli L (1980) An Approach to Neuroanatomical and Neurochemical Psychophysiology. Press of Scaravaglio Bros., Turin.

Van Brussel GHA (1993) Is cannabis altijd een 'soft drug'? Nederlands Tijdschrift voor Geneeskunde, 137, 1081-1083.

Van Dellen HJ, Aasman J, Mulder LJM and Mulder G (1985) Statistical versus spectral measures of heart rate variability. In: The Psychophysiology of Cardiovascular Control (Eds JF Orlebeke, G Mulder and LPJ van Doornen), pp. 353-374. Plenum Press, New York.

Van der Wal HJ (1985) Roken, Drinken, Cannabisgelmuik. Stichting voor Wetenschappelijk Onderzoek van Alcohol- en Druggebruik, Utrecht, The Netherlands.

Van Laar MW, Volkerts ER and Willigenburg APP (1992) Therapeutic effects and effects on actual driving performance of chronically administered buspirone and diazepam in anxious outpatients. Journal of Clinical Psychopharmacology, 12, 86-95.

Van Ree F and Esseveld P (1985) Drugs: De Medische en Maatschappelijke Aspecten. Het Spectrum, Utrecht. 
Van Veggel LMA and O'Hanlon JF (1993) Effects of Ondansetron and Diazepam on Driving and Psychometric Performance in Healthy Volunteers. Technical Report No. IGVG 93-26, Institute for Drugs, Safety and Behavior, University of Limburg, Maastricht, The Netherlands.

Varma VK, Malhotra AK, Dang R, Das K and Nehra R (1988) Cannabis and cognitive functions: A prospective study. Drug and Alcobol Dependence, 21, 147-152.

Venkoba Rao A, Rawlin CR, Pradeep D and Rajgopal P (1975) Cannabis (Ganja) and cognition. Indian Journal of Psychiatry, 17, 233-237.

Vermeeren A, Swijgman HF and O'Hanlon JF (1993) Acute and Subchronic Effects of Alpidem, Lorazepam and Placebo on Anxiety, Psychometric Test Performance and Actual Driving in Anxious Patients. Technical Report No. IGVG 93-19, Institute for Drugs, Safety and Behavior, University of Limburg, Maastricht, The Netherlands.

Vincent BJ, McQuiston DJ, Einhorn LH, Nagy CM and Brames MJ (1983) Review of cannabinoids and their antiemetic effectiveness. Drugs, 25(Suppl 1), 52-62.

Vinciguerra V, Moore TMSW and Brennan E (1988) Inhalation marijuana as an antiemetic for cancer chemotherapy. New York State Journal of Medicine, 88, 525-527.

Volkerts ER, Abbink F, Van Laar MW, Plomp PR and Maes RAA (1988) Comparison of the Effects of Ritanserin, $5 \mathrm{mg}$, b.i.d. and Lorazepam $1.5 \mathrm{mg}$, b.i.d., upon Driving Performance in an Over-the-Road Driving Test. Technical Report No. 11-88, Netherlands Institute for Drugs and Doping Research, University of Utrecht, Utrecht, The Netherlands.

Volkow ND, Gillespie H, Mullani N, Tancredi L, Grant C, Ivanovic M and Hollister L (1991) Cerebellar metabolic activation by delta-9-tetrahycrocannabinol in human brain: A study with positron emission tomography and ${ }^{18} \mathrm{~F}-2$-fluoro-2-deoxyglucose. Psychiatry Research: Neuroimaging, 40, 69-78.

Wall ME, Sadler BM, Brine D, Taylor H and Perez-Reyes M (1983) Metabolism, disposition and kinetics of delta-9-tetrahydrocannabinol in men and women. Clinical Pharmacology and Therapeutics, 34, 352-363.

Warren RA, Simpson HM, Hilchie J, Cimbura G, Lucas D and Bennett R (1981) Drugs detected in fatally injured drivers in the Province of Ontario. In: Alcobol, Drugs and Traffic Safety. Proceedings, 8th International Conference on Alcohol, Drugs and Traffic Safety, June 15-19, 1980, Stockholm, Sweden (Ed L Goldberg), pp. 203-217. Almqvist and Wiksell International, Stockholm.

Weil AT (1970) Adverse reactions of marihuana: Classification and suggested treatment. The New England Journal of Medicine, 282, 997-1000.

Weil AT, Zinberg NE and Nelson JM (1968) Clinical and psychological effects of marijuana in man. Science, 162, 1234-1242.

Wert RC and Raulin ML (1986) The chronic cerebral effects of cannabis use: Psychological findings and conclusions. International Journal of the Addictions, 21, 629-642.

Wesemann P, Twisk DAM and Vis AA (1989) Covernota bij het state-of-the-art onderzoek geneesmiddelen en drugs. Report No. R-89-37, Stichting Wetenschappelijk Onderzoek Verkeersveiligheid, Leidschendam.

Williams AF, Peat MA, Crouch DJ, Wells JK and Finkle BS (1985) Drugs in fatally injured young male drivers. Public Health Reports, 100, 19-25.

Wu T-C, Tashkin DP, Dhajed B and Rose JE (1988) Respiratory hazards of smoking marijuana as compared with tobacco. The New England Joumal of Medicine, 318, 347-351.

Yager J, Crumpton E and Rubenstein R (1983) Flashbacks among soldiers discharged as unfit who abused more than one drug. American Joumal of Psychiatry, 140, 857-861. 
Yesavage JA, Leirer VO, Denari M and Hollister LE (1985) Carry-over effects of marijuana intoxication on aircraft pilot performance: A preliminary report. American Journal of Psychiatry, 142, 1325-1329.

Zacny JP and Chait LD (1989) Breathhold duration and response to marijuana smoke. Pharmacology, Biochemistry and Behavior, 33, 481-484.

Zacny JP and Chait LD (1991) Response to marijuana as a function of potency and breathhold duration. Psychopharmacology, 103, 223-226.

Zijlstra FRH and Van Doorn L (1985) The Construction of a Scale to Measure Perceived Effort. Technical Report, University of Delft, Delft, The Netherlands.

Zinberg NE (1979) On cannabis and health. Journal of Psychedelic Drugs, 11, 135-144.

Zuardi AW, Shirakawa I, Finkelfarb E and Karniol IG (1982) Action of cannabidiol on the anxiety and other effects produced by $\Delta^{2}-\mathrm{THC}$ in normal subjets. Psychopharmacology, 76, 245-250.

Zuardi AW, Cosme RA, Graeff FG and Guimarães FS (1993) Effects of ipsapirone and cannabidiol on human experimental anxiety. Journal of Psychopharmacology, 7, 82-88.

Zuckerman B and Bresnahan K (1991) Developmental and behavioral consequences of prenatal drug and alcohol exposure. The Pediatric Clinics of North America, 38, 1387-1406.

Zuckerman B, Frank DA, Hingson R, Amaro H, Levenson SM, Kayne H, Parker S, Vinci R, Aboagye K, Fried LE, Cabral H, Timperi R and Bauchner H (1989) Effects of maternal marijuana and cocaine use on fetal growth. The New England Journal of Medicine, 320, 762-768. 


\section{DANKWOORD}

Aan de totstandkoming van dit proefschrift hebben vele mensen een bijdrage geleverd. Allereerst denk ik dan aan alle proefpersonen, meer dan zeventig in getal, die bereid waren om aan het onderzoek deel te nemen. Mede dankzij hen heb ik vele plezierige herinneringen aan het onderzoek overgehouden.

Daarnaast wil ik een aantal mensen met name noemen. Allereerst natuurlijk mijn begeleider en co-promotor Jim O'Hanlon, directeur van het Instituut voor Humane Psychofarmacologie. Ik ben hem zeer erkentelijk voor de gedegen en kritische begeleiding. Hij was de grote animator achter het onderzoek en stond altijd open voor overleg en discussie. De door mij aangeleverde delen van het proefschrift heeft hij altijd van deskundig commentaar voorzien. Ik dank hem voor de prettige samenwerking.

Ik ben Marcel van den Hout, hoogleraar Geestelijke Gezondheidkunde bij de vakgroep Differentiële en Experimentele Psychologie, zeer erkentelijk voor het feit dat hij genegen was om als mijn promotor op te treden. Zijn bijdrage aan het proefschrift is voor mij zeer waardevol geweest. De bezieling die van hem uitging werkte zeer motiverend; de snelheid waarmee hij de stukken van commentaar voorzag heb ik zeer gewaardeerd. Ik hoop dat onze relatie mag resulteren in verdergaande samenwerking.

Het onderzoek had echter nooit uitgevoerd kunnen worden zonder de toestemming van verschillende instanties. Ten eerste denk ik dan aan het Amerikaanse 'Department of Transportation' en het Nederlandse Ministerie van Verkeer en Waterstaat. Met name wil ik Ted Anderson bedanken voor het initiëren van het onderzoekprogramma, en Jim Frank en Jan Busstra voor hun rol als 'monitor' voor resp. het Amerikaanse en Nederlandse Ministerie. Daarnaast gaat mijn dank uit naar de Hoofdofficier van Justitie in Maastricht, mr. J.H. Fransen, wiens toestemming voor het onderzoek van essentieel belang was. Als gevolg van de besprekingen met hem is er een onafhankelijke begeleidingscommissie ingesteld die er voor diende te waken dat het onderzoek zorgvuldig zou worden opgezet en uitgevoerd, overeenkomstig de medische en wettelijke veiligheidsregels. Deze commissie bestond uit: $\mathrm{mr}$. J. Oosterhof, verkeersschout te Maastricht, later vervangen door diens opvolger dhr. J. Bardoul, dr. H.C. 
Milius, psychiater en lid van de Medisch Ethische Commissie van de Rijksuniversiteit Limburg, en drs. J. Tjoeng, Inspecteur van de Volksgezondheid voor de Geneesmiddelen in Noord-Brabant en Limburg. Ik wil de commissieleden hartelijk bedanken voor hun bijdragen en prettige samenwerking. Tenslotte wil ik ook het Ministerie van WVC bedanken voor het afgeven van het opiumverlof en de vergunningen voor het importeren van marihuana sigaretten, en de Medisch Ethische Commissie van de Rijksuniversiteit Limburg voor het behandelen en goedkeuren van de onderzoeksaanvragen.

Ik wil ook allen bedanken die een bijdrage geleverd hebben aan de uitvoering van het onderzoek. De afdeling KNO van het Academisch Ziekenhuis Maastricht, en met name Herman Kingma, bedank ik voor de geboden gastvrijheid tijdens de laboratorium studie. Verder wil ik allen die betrokken zijn geweest bij het afsluiten van de A76 tijdens de eerste rijstudie hartelijk bedanken voor hun medewerking. In het bijzonder wil ik hierbij noemen de heren: P.G.S.M. Geurts, Verkeersadviseur van het Korps Rijkspolitie District Limburg, Ing. F.V.J.M. Schepers, Hoofd Buro Wegendienstkring Zuid, Afdeling Verkeer en Vervoer, en Ing. H.P.A. Thegels, het toenmalige hoofd van de Technische Dienst van Rijkswaterstaat, dienstkring St. Joost.

De analyses van de bloedmonsters werden verricht door Prof.dr. M.R. Möller en zijn medewerkers van het Institut für Rechtsmedizin van de Universiteit van Saarland te Homburg. Ik dank hen voor de belangrijke samenwerking.

De rijinstructeurs, die tijdens de rijtesten borg stonden voor de (verkeers)veiligheid, wil ik hartelijk bedanken voor hun grote inzet; met name onze 'vaste' rijinstructeur Henk Brauers, op wie ik altijd kon rekenen.

Verder wens ik al mijn collega's en oud-collega's te bedanken voor de prettige sfeer waarin ik heb mogen werken. Ik wil Han de Gier bedanken voor zijn aandeel in de voorbereidingsfase van het onderzoek. Vic Bonke en Marius Bassie dank ik voor hun morele ondersteuning. Annemiek Vermeeren en Wim Riedel, mijn paranimfen, wil ik hartelijk bedanken voor het feit dat ik altijd bij hen terecht kon, met 'wetenschappelijke' problemen of zomaar om m'n hart te luchten. Ik dank ook hen die voor de medische, technische en secretariële ondersteuning hebben gezorgd: Martin van Boxtel, Astrid van Houte, Marcel Hendrickx, Véronique van der Varst en Susanne Aldenhoff. Ook alle assistenten, die mij hebben geholpen bij de data-collectie, ben ik zeer erkentelijk voor hun grote inzet.

Tenslotte wil ik familie en vrienden bedanken voor de interesse die ze in mijn werk hebben getoond. Lieve Greetje, bovenal bedank ik jou voor je geduld en je voortdurende steun in de afgelopen periode. 


\section{CURRICULUM VITAE}

Hindrik Robbe werd geboren op 16 februari 1962 te Stadskanaal. In 1980 behaalde hij het gymnasium- $\beta$ diploma aan het Ubbo Emmius Lyceum in Stadskanaal. In datzelfde jaar begon hij aan de studie Psychologie aan de Rijksuniversiteit Groningen. Na het kandidaatsexamen volgde hij de afstudeerrichting functieleer, waarbij zijn interesse voornamelijk uitging naar de psychofysiologie. In juni 1987 slaagde hij cum laude voor het doctoraalexamen. Sinds augustus 1987 is hij als onderzoeker verbonden aan het Instituut voor Humane Psychofarmacologie (v/h IGVG) van de Rijksuniversiteit Limburg te Maastricht, alwaar hij onderzoek verricht naar de effecten van geneesmiddelen en andere psychotrope stoffen op menselijk gedrag. 


\section{INFLUENCE OF MARIJUANA ON DRIVING HWJ ROBBE}

The role of cannabis in traffic accident causality is still unknown. That cannabis is often detected in crashinvolved drivers could either indicate a causal role or simply reflect the fact that it is the most used illicit drug. One might assume a causal relationship if it were definitely known that cannabis, in doses regularly taken by users who drive, severely impairs their ability to operate a vehicle. Yet results collected in various laboratory and driving simulation tests have provided conflicting results leading to a controversy. Given the societal relevance of the issue and the persistence of the controversy, it seemed timely to investigate cannabis' effects on driving performance in the real traffic environment.

This book contains a comprehensive literature review concerning many aspects of cannabis, including its effects on driving performance and traffic safety. It continues with a description of one laboratory and three actual driving studies that progressively approached reality. These were conducted on a closed section of a primary highway, on a primary highway in the presence of other traffic, and in urban traffic. Together, they provide an answer to the questions addressed by the book: does cannabis impair driving performance, and if so, how much in comparison. with other psychoactive drugs.

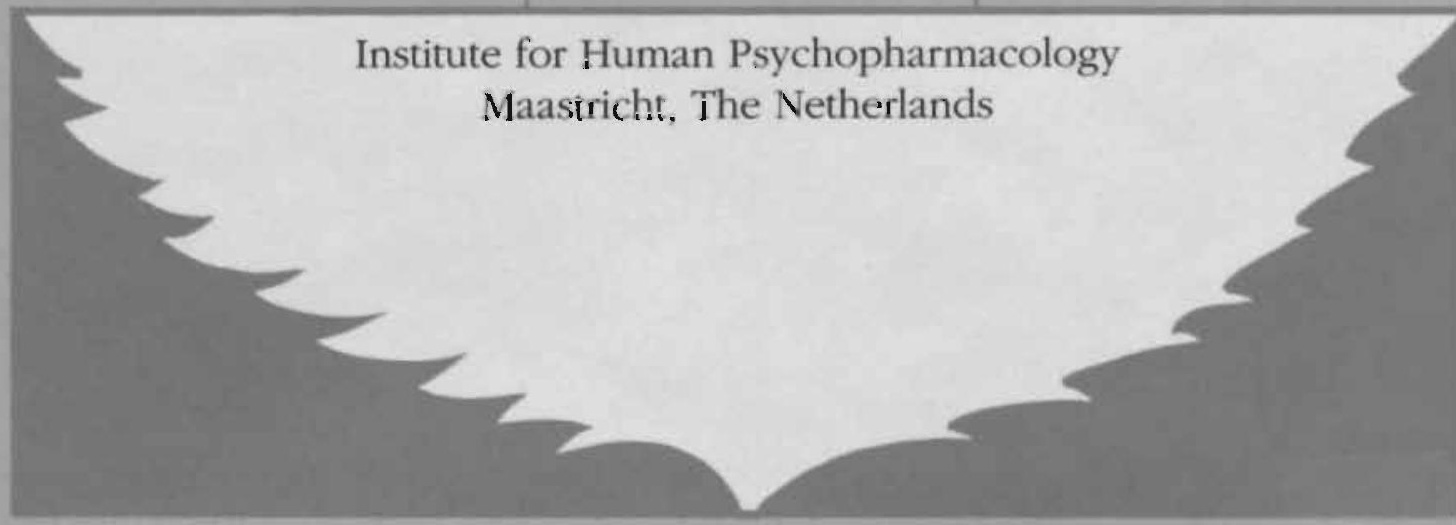

